\title{
Advanced Control Strategies for a 6 DoF Hydraulic Parallel Robot Based on the Dynamic Model
}

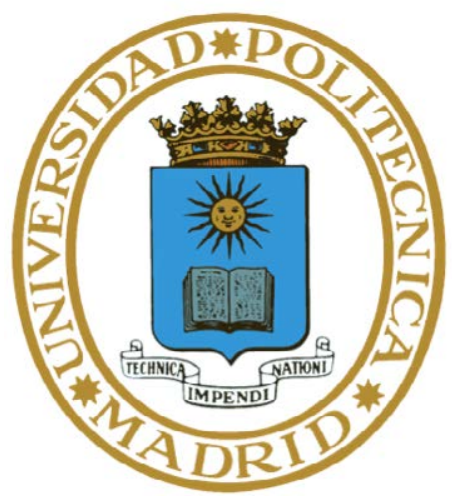

Lisandro José Puglisi

Escuela Técnica Superior de Ingenieros Industriales Universidad Politécnica de Madrid

A thesis submitted for the degree of PhilosophiceDoctor (PhD) in Automatics and Robotics. 
1. Reviewer:

2. Reviewer:

3. Reviewer:

4. Reviewer:

5. Reviewer:

Day of the defense:

Signature from head of $\mathrm{PhD}$ committee: 


\begin{abstract}
Nowadays robots have made their way into real applications that were prohibitive and unthinkable thirty years ago. This is mainly due to the increase in power computations and the evolution in the theoretical field of robotics and control.

Even though there is plenty of information in the current literature on this topics, it is not easy to find clear concepts of how to proceed in order to design and implement a controller for a robot.

In general, the design of a controller requires of a complete understanding and knowledge of the system to be controlled. Therefore, for advanced control techniques the systems must be first identified. Once again this particular objective is cumbersome and is never straight forward requiring of great expertise and some criteria must be adopted.

On the other hand, the particular problem of designing a controller is even more complex when dealing with Parallel Manipulators (PM), since their closed-loop structures give rise to a highly nonlinear system.

Under this basis the current work is developed, which intends to resume and gather all the concepts and experiences involve for the control of an Hydraulic Parallel Manipulator.

The main objective of this thesis is to provide a guide remarking all the steps involve in the designing of advanced control technique for PMs.

The analysis of the PM under study is minced up to the core of the mechanism: the hydraulic actuators. The actuators are modeled and experimental identified. Additionally, some consideration regarding traditional PID controllers are presented and an adaptive controller is finally implemented. From a macro perspective the kinematic and dynamic model of the PM are
\end{abstract}


presented. Based on the model of the system and extending the adaptive controller of the actuator, a control strategy for the PM is developed and its performance is analyzed with simulation. 
To Ce and Elena...

las flores mas dulces de mi jardin.. 


\section{Acknowledgements}

As a scholar of the MAEC-AECID scholarship program, I would like to express my gratitude to the $M A E C$ - $A E C I D$ for given me the opportunity to study at this university and also to UPM-Santander scholarship.

I would like to thanks to my tutor D.Roque Saltaren for his trust, confidence, and support. I only have words of admiration and gratitude for you.

I would like to thanks to my cotutor Da. Cecilia Garcia for her advice during all these years.

I would like to thanks to D. Rafael Aracil for his support, and for given me the opportunity to be part of the Group of Robots and Intelligent Machines.

To all my colleges from the DISAM, that are always willing to help. Thanks a lot to all of you. To Carol and Nico that are always near when a friend needs them. To Jesus for his support and friendship.

I would like to thanks to my family, for their unconditional love and support. I owe all to you.

And at last but not the least, to $C e$ for everything, it wouldn't be possible without you... 
The development of this thesis was supported by Ministerio de Asuntos Exteriores y de Cooperación (MAEC) y de la Agencia Española de Cooperación Internacional para el Desarrollo (AECID), under the scholarships : MAEC-AECID 2009/2013 Programa II-E. and Programa II-A. 


\section{Contents}

List of Figures $\quad$ xi

List of Tables $\quad$ xv

1 Motivation and Aims 1

1.1 Introduction . . . . . . . . . . . . . . . . . . 1

1.2 Aims of this work . . . . . . . . . . . . . . 2

1.3 Contributions of this work . . . . . . . . . . . . 3

1.4 Structure of this Document . . . . . . . . . . . . . . 5

$\begin{array}{ll}\text { I Basic Concepts } & 7\end{array}$

2 Parallel Mechanism $\quad 9$

2.1 Introduction . . . . . . . . . . . . . . . . . . 9

2.2 Parallel Robots . . . . . . . . . . . . . . . . . . . . . 9 9

2.3 Historical Review . . . . . . . . . . . . . . . . . . . . . . 11

2.4 Parallel Robots and their applications . . . . . . . . . . . . . . . 12

2.5 Kinematic Formulation . . . . . . . . . . . . . . . . . 16

2.5.1 Position Modeling . . . . . . . . . . . . . . 17

2.5.2 Instantaneous or Velocity Modeling . . . . . . . . . . . . . 18

2.5.2.1 Jacobian Matrix Based on Screw Theory . . . . . . . 18

2.6 Kinematics Analysis . . . . . . . . . . . . . . . . . . . . . . 19

2.7 Dynamic Modeling . . . . . . . . . . . . . . . . . . . . . . . 21

2.7.1 Virtual Work Formulation . . . . . . . . . . . . . . . 23

2.7.2 Task Space and Joint Space Dynamics . . . . . . . . . . . . . 23 
3 Hydraulic Systems $\quad 25$

3.1 Introduction . . . . . . . . . . . . . . . . . . . 25

3.2 Hydraulic Actuation Systems _ . . . . . . . . . . . . . . 25

3.3 Brief Description of Hydraulic Elements . . . . . . . . . . . . . . . 27

3.3.1 Hydraulic Power Station . . . . . . . . . . . . . . . . . . 27

3.3 .2 Valves . . . . . . . . . . . . . . . . . . . . . . 28

3.3.3 Actuators . . . . . . . . . . . . . . . . . . . . 31

3.4 Implementation of Hydraulic Actuation Systems . . . . . . . . . . . 33

II Hydraulic Actuator 35

4 Servo Hydraulic Actuator $\quad 37$

4.1 Introduction . . . . . . . . . . . . . . . . . 37

4.2 Servo Hydraulic Actuator Description _ . . . . . . . . . . . . 39

4.2 .1 Servo-valve . . . . . . . . . . . . . . . . . . . 40

4.2 .2 Asymmetric double effect cylinder . . . . . . . . . . . . . . 40

4.3 Simplified Non linear Model . . . . . . . . . . . . . . . . . . . . 40

4.4 Linearized Model . . . . . . . . . . . . . . . . . . . . . . . . . 44

4.4 .1 Model Analysis . . . . . . . . . . . . . . . . . . . . . . . 49

4.5 Conclusions . . . . . . . . . . . . . . . . . . . 51

$5 \quad$ Experimental Identification of SHA

5.1 Introduction . . . . . . . . . . . . . . . . . . . . . 53

5.2 Identification Process . . . . . . . . . . . . . . . . . . . 54

$5.2 .1 \quad$ Experiment Design . . . . . . . . . . . . . . . . 56

5.2 .2 Closed Loop Transfer Function Identification . . . . . . . . . . 57

5.2 .3 Dynamic Motion Parameters Identification . . . . . . . . . . 57

5.2 .4 Sensibilities Constants Optimization . . . . . . . . . . . 58

5.3 Experimental Setup . . . . . . . . . . . . . . . . . . . 58

5.4 Experimental identification . . . . . . . . . . . . . 60

5.4.1 Experiment \#1: PRBS response under closed loop . . . . . . . 60

5.5 Conclusions . . . . . . . . . . . . . . . . . . . 65 
6 Experimental Friction Model Identification $\quad 69$

6.1 Introduction . . . . . . . . . . . . . . . . . . 69

6.2 Friction effects observed in the SHA . . . . . . . . . . . 70

6.2.1 Oscillations - Limit cycles . . . . . . . . . . . . . 70

6.3 LuGre Model Friction . . . . . . . . . . . . . . . . . . . . 71

6.4 Experimental Identification of LuGre Model Friction . . . . . . . . . . . 73

6.4.1 Steady State Regime . . . . . . . . . . . . . . . . 73

6.4.1.1 Experiment \#1: Constant Velocity Motions . . . . . . . 73

6.4 .2 Pre-sliding regime . . . . . . . . . . . . . . . . 75

6.4.2.1 Experiment \#2: Force Control Response . . . . . . . 77

6.5 Conclusions . . . . . . . . . . . . . . . . . . 80

$\begin{array}{llr}7 & \text { Control Strategies SHA } & 83\end{array}$

7.1 Introduction . . . . . . . . . . . . . . . . 83

7.2 Control Strategies implemented in the SHA . . . . . . . . . . . 84

7.3 A brief discussion on the controller design . . . . . . . . . . . . 87

7.4 PI Controller with Robust Stability . . . . . . . . . . . . . . . . 91

7.4.1 Nominal Plant and Uncertainties Definitions . . . . . . . . . . 93

7.4.1.1 Unstructured uncertainty definition . . . . . . . . 93

7.4 .2 Controller . . . . . . . . . . . . . . . . . 94

7.4 .3 Robustness Analysis . . . . . . . . . . . . . . . 95

7.4.3.1 Internal Stability (Nominal Stability) . . . . . . . . 95

7.4.3.2 Robust Stability . . . . . . . . . . . . . . 97

7.4.3.3 Robust Performance . . . . . . . . . . . . . . . 99

7.5 Adaptive Hybrid Force-Position Controller . . . . . . . . . . . . . . . 100

7.5.1 Force-Position Controller Description . . . . . . . . . . . . . . . 102

7.5.1.1 Position PI Controllers tuning . . . . . . . . . . . . 102

7.5.1.2 Controller Implementation . . . . . . . . . . . . 103

7.5 .2 Friction Estimation . . . . . . . . . . . . . . . . . . . 104

7.5.3 External Load Mass Estimation . . . . . . . . . . . . . . . 105

7.5.4 Indirect Measure of Load Pressure . . . . . . . . . . . . . . . 106

7.5.5 Inverse Dynamics . . . . . . . . . . . . . . . . . . 106

7.5.6 Velocity and Acceleration estimation . . . . . . . . . . . 108 
7.6 Experiments . . . . . . . . . . . . . . . . . . 110

7.6.1 Experiment \# 1: Step response . . . . . . . . . . . . . . . 110

7.6.2 Experiment \# 2: Position Reference Tracking . . . . . . . . . . . 111

7.6.3 Experiment \# 3: Robustness to external perturbations . . . . . . 112

7.7 Conclusions . . . . . . . . . . . . . . . . . . . . . 114

III Hydraulic Parallel Manipulator 119

8 Hydraulic 6 DoF Parallel Robot 121

8.1 Introduction . . . . . . . . . . . . . . . . . . . . . . . 121

8.2 Description of the SG Mechanism . . . . . . . . . . . . . . . . . 122

8.2 .1 Mechanical Design . . . . . . . . . . . . . . . . . . . . . 122

8.2.2 Hydraulic Actuation System Design . . . . . . . . . . . . . 124

8.2.3 Electronics and Sensorization . . . . . . . . . . . . . . 125

8.3 Simulation Environment . . . . . . . . . . . . . . . . . 126

8.4 Conclusions . . . . . . . . . . . . . . . . . . . 127

9 Kinematic Analysis $r 29$

9.1 Introduction . . . . . . . . . . . . . . . . . . . . . . . 129

9.2 Inverse Kinematics ～. . . . . . . . . . . . . . . . . . . 130

9.3 Workspace . . . . . . . . . . . . . . . . . . . . 130

9.4 Direct Kinematics . . . . . . . . . . . . . . . . . . . . . . . . 134

9.4.1 Direct Kinematics Approach . . . . . . . . . . . . . . 135

9.4.2 Constraint vector definition . . . . . . . . . . . . . 137

9.4.3 Jacobian matrix of the constraint vector . . . . . . . . . . . . 138

9.4.4 Performance Evaluation . . . . . . . . . . . . . . . . . . . . . . 140

9.5 Instantaneous Kinematics ． . . . . . . . . . . . . . . . . . . 143

9.6 Singular Configurations _ . . . . . . . . . . . . . . . . . 145

9.7 Conclusions . . . . . . . . . . . . . . . . . . . . . 146

$\begin{array}{ll}10 \text { Dynamic Analysis } & 147\end{array}$

10.1 Introduction . . . . . . . . . . . . . . . . . . . . . . . . . . 147

10.2 Dynamic Modeling of PMs . . . . . . . . . . . . . . . . . 148

10.3 Dynamic Model Based on Virtual Work Principle . . . . . . . . . . . . . 149 
10.4 Model Validation . . . . . . . . . . . . . . . . . . . . . 154

10.4.1 Simulations . . . . . . . . . . . . . . . . . 154

10.5 Conclusions . . . . . . . . . . . . . . . . . . . 157

$\begin{array}{lr}11 \text { Control Strategies for SG } & 159\end{array}$

11.1 Introduction . . . . . . . . . . . . . . . . . . . . . 159

11.2 Controllers for Hydraulic Hexapode PM . . . . . . . . . . . . . 160

11.3 Trajectory Generation . . . . . . . . . . . . . . . . 161

11.4 Controllers . . . . . . . . . . . . . . . . . . . . . . 164

11.4.1 Decoupled Controllers . . . . . . . . . . . . . . . . . . . 164

11.4.2 Dynamic Model Based Controller . . . . . . . . . . . . . . . 165

11.5 Simulation and Results . . . . . . . . . . . . . . . . . . . 166

11.6 Conclusions . . . . . . . . . . . . . . . . . . . 168

$\begin{array}{lr}12 \text { Conclusions } & 169\end{array}$

$\begin{array}{ll}\text { A Parameters of the Identification of the HSA } & 173\end{array}$

B Well-posedness of the feedback System 175

$\begin{array}{ll}\text { C Virtual rotations and Euler parameters } & 177\end{array}$

$\begin{array}{lr}\text { D Estimation of the Dynamics of theTestbed } & 179\end{array}$

E Results from the validation of the dynamic model of the PM 185

$\begin{array}{lr}\text { Bibliography } & 193\end{array}$ 
CONTENTS 


\section{List of Figures}

2.1 Schematic diagram of a general PM. . . . . . . . . . . . . . 10

2.2 Some of the first implementations of parallel robots. . . . . . . . . . . 12

2.3 Delta PM . . . . . . . . . . . . . . . . . . . . . . . . . . . . 13

2.4 Comertial solutions using Delta robots. . . . . . . . . . . . . . 13

2.5 Commercial hexapods. . . . . . . . . . . . . . . . . . . . . . 14

2.6 Applications of hexapods PM . . . . . . . . . . . . . 15

2.7 Spherical Parallel Mechanism . . . . . . . . . . . . . . . . . . 16

2.8 PM's workspace determination . . . . . . . . . . . . . . 20

3.1 Schematic diagram of a general hydraulic system . . . . . . . . . . 26

3.2 Positive displacement pump concept . . . . . . . . . . . . . . 28

3.3 Displacement pumps family tree . . . . . . . . . . . . . . . . . . . 29

3.4 Flow through an orifice . . . . . . . . . . . . . . . . . . 29

3.5 Fluid control valves. . . . . . . . . . . . . . . . . . . . . . . 30

3.6 Proportional Servo-valve. . . . . . . . . . . . . . . . . . . . . . . 31

3.7 Schematic diagram of the internal chamber of the cylinder. . . . . . . . 33

3.8 Used of hydraulic systems. . . . . . . . . . . . . . . . . . . . 34

3.9 Implementation of hydraulic actuation on innovation. . . . . . . . . . . 34

4.1 Schematic diagram of the servo hydraulic actuator circuit. . . . . . . . . 39

4.2 Proportional Servo-valve. . . . . . . . . . . . . . . . . . . . . . . 40

5.1 Identification Process . . . . . . . . . . . . . . . . . . . . 55

5.2 Experimental Setup. . . . . . . . . . . . . . . . . . . 59

5.3 Input-output experimental data . . . . . . . . . . . . . . 61 


\section{LIST OF FIGURES}

5.4 A typical result from the identified sets of parameter associated with the motions dynamics for no external load. . . . . . . . . . . . . . 62

5.5 Parameters of the system. . . . . . . . . . . . . . . . . . . 63

$5.6 G_{o l}$ pole-zero diagram $\ldots \ldots \ldots \ldots$. . . . . . . . . . 64

5.7 Identified sensibility constants . . . . . . . . . . . . . . 65

5.8 Pole-zero diagram of $P(s)=\Delta Y / \Delta X \ldots \ldots \ldots 6$

5.9 Simulated response of the identified model. . . . . . . . . . . . . . 66

5.10 Simulink model of the identified HSA . . . . . . . . . . . . . 67

6.1 Oscillations caused by friction. . . . . . . . . . . . . . . . . 71

6.2 Detail of the oscillations. . . . . . . . . . . . . . . . . 72

6.3 Step response in open loop . . . . . . . . . . . . . . 75

6.4 Friction force characteristic of the SHA _ . . . . . . . . . . . 76

6.5 Curves obtained from a force control test. . . . . . . . . . . . . . . . . . 79

6.6 Force-displacement chart graph in pre-sliding regime. . . . . . . . . . . 80

7.1 Time response with classic controllers . . . . . . . . . . . . . . 88

7.2 Steady state analysis . . . . . . . . . . . . . . . . . . 89

7.3 Schematic diagram for a generic internal stability analysis. . . . . . . . . 90

7.4 Multiplicative uncertainty definition . . . . . . . . . . . . . . 94

7.5 PI Controller . . . . . . . . . . . . . . . . . . . . . 95

7.6 Schematic diagram for Internal Stability Analysis . . . . . . . . . . . . 96

7.7 Nyquist diagram for $\Pi(s) \ldots \ldots \ldots \ldots$

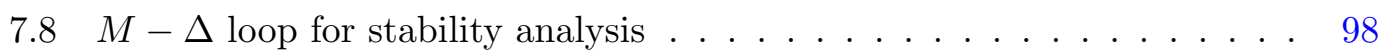

7.9 Multiplicative perturbed system . . . . . . . . . . . . . . . . . . . 99

7.10 Simulink model for the Adaptative Hybrid Controller . . . . . . . . . . . 101

7.11 Local force-position controller . . . . . . . . . . . . . . . . . . . . . 102

7.12 PI controller time response . . . . . . . . . . . . . . . . 103

7.13 Adaptative Controller . . . . . . . . . . . . . . . . . . . . 105

7.14 Load mass estimation _. . . . . . . . . . . . . . . . . . . 107

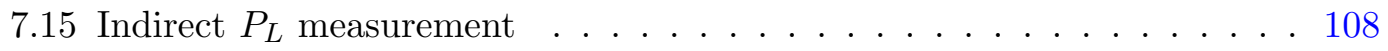

7.16 Step response of the system for different working set point. . . . . . . . 111

7.17 Reference tracking with PI controller . . . . . . . . . . . . . . . 113

7.18 Reference tracking with Adaptive controller . . . . . . . . . . . . . . . . 114 
7.19 Frequency error dependency . . . . . . . . . . . . . . . . . 115

7.20 PI controller response under external perturbations . . . . . . . . . . 116

7.21 Adaptive controller response under external perturbations . . . . . . . . 117

8.1 CAD of the 6 UPS mechanism . . . . . . . . . . . . . . 122

8.2 Universal joint details . . . . . . . . . . . . . . . . . . 123

8.3 Spherical joint details . . . . . . . . . . . . . . . . . . . 124

8.4 Power Hydraulic Station. . . . . . . . . . . . . . . . . . . . 125

8.6 Simulation environment . . . . . . . . . . . . . . . 127

9.1 Schematic diagram of the mechanism 6UPS. . . . . . . . . . . . . . 131

9.2 6UPS maximum dexterous workspace . . . . . . . . . . . . . . 132

9.3 Maximum pure translation . . . . . . . . . . . . . . . . 133

9.4 6Uㅗ maximum pure translations workspace representation with both platform parallel. . . . . . . . . . . . . . . . . 133

9.5 6UㅗS maximum pure translations workspace representation with both platform parallel. 9.5(a): slice of the workspace with plane $z=600$. 9.5(b): slice of the workspace with plane $y=0$.

9.6 6UPS maximum rotation workspace representation. Solid volume representation. . . . . . . . . . . . . . . . . . . . . . 134

9.7 6UㅗS maximum rotation workspace representation. 9.7(a): slice of the workspace with plane $Y a w=0^{\circ}$. 9.7(b): slice of the workspace with plane Roll $=0^{\circ}$. . . . . . . . . . . . . . . . . . . . . 135

9.8 Direct Kinematics Algorithm . . . . . . . . . . . . . . . . . . 136

9.9 Schematic diagram of a two-links leg of a generic PM. . . . . . . . . . 138

9.10 Evaluation Procedure (2 and 4 are symbolic expression). . . . . . . . . 142

9.116 UPS orientation workspace and singular configurations for $\boldsymbol{r}=[0,0,700]$. Solid volumen representation for the workspace and singular configurations surface. . . . . . . . . . . . . . . . . . . . . . 145

9.126 UPS orientation workspace and singular configurations for $\boldsymbol{r}=[0,0,700]$. : slice with plane $R o l l=-30^{\circ}$. : slice with plane $R o l l=0^{\circ}$. : slice with plane Roll $=30^{\circ}$. . . . . . . . . . . . . . . . . . . . . 146

10.1 Schematic diagram of 6 UPS. . . . . . . . . . . . . . 151 


\section{LIST OF FIGURES}

10.2 Schematic diagram for the dynamic model validation . . . . . . . . 155

10.3 Absolute and relative maximum error for all the simulated trajectories . 157

11.1 Velocity profile . . . . . . . . . . . . . . . . . 162

11.2 Movement 1: Point to point movement. . . . . . . . . . . . . 163

11.3 Movement II: Circular trajectory . . . . . . . . . . . . . . . . . . . . . . 164

11.4 Schematic diagram for the SG joint space controller . . . . . . . . . 165

11.5 Implementation of the adaptive controller of the SHA in the SG . . . 165

11.6 Joint space controller with feedforward comensation . . . . . . . . . 166

11.7 Joint space controller with feedforward comensation . . . . . . . . . 166

11.8 End effector position during the diagonal movement. . . . . . . . . . 167

11.9 End effector position during circular movement. . . . . . . . . . . . 168

D.1 Force-velocity characteristic of the linear guide. . . . . . . . . . . . 180

D.2 Estimation of the parameter of the Force-velocity model of the linear guide. . . . . . . . . . . . . . . . . . . . . . . 181

D.3 Force-velocity estimation based on friction model. . . . . . . . . . . . . . 182

D.4 Force-velocity estimation based on unbounded spline interpolation. . . . 183

D.5 Force-velocity characterization of the linear guide . . . . . . . . . . . . 184

E.1 Dynamic Model Validation, trajectory Tx . . . . . . . . . . . . . 186

E.2 Dynamic Model Validation, trajectory Ty . . . . . . . . . . . . . . . 187

E.3 Dynamic Model Validation, trajectory $\mathrm{Tz}$. . . . . . . . . . . . . . . . 188

E.4 Dynamic Model Validation, trajectory Rx ～. . . . . . . . . . . . . 189

E.5 Dynamic Model Validation, trajectory Ry ～. . . . . . . . . . . . . 190

E.6 Dynamic Model Validation, trajectory Rz . . . . . . . . . . . . . . . 191 


\section{List of Tables}

6.1 Estimated parameters for the friction model in steady state regime. . . . 76

6.2 Pre-sliding parameters estimation. . . . . . . . . . . . . . . 80

7.1 Controllers and well-posedness of the feedback system. (see B for the complete Table). . . . . . . . . . . . . . . . . . . . . . . 91

7.2 PI Controllers . . . . . . . . . . . . . . . . . . . . . . . . 104

8.1 Elements of the 6 UPS mechanism . . . . . . . . . . . . . . . . 124

9.1 Performance evaluation of the method. . . . . . . . . . . . . . 143

10.1 Dynamic properties of the PM . . . . . . . . . . . 155

10.2 Simulation Results . . . . . . . . . . . . . . . . 156

A.1 Parameters of motion . . . . . . . . . . . . . . 173

A.2 Sensibility Constants . . . . . . . . . . . . . . . . . . 174

A.3 Parameter of the Transfer Functions . . . . . . . . . . . . . . . . 174

B.1 Controllers and well-posedness of the feedback system. . . . . . . . 176

D.1 Parameters estimated for the force-velocity model of the linear guide . . 182 


\section{1}

\section{Motivation and Aims}

"... if you take the blue pill the history ends, you wake up in your bed and believe whatever you want to believe, you take the red pill and you stay in wonderland and I show you how deep the rabbit hole goes ..."

Morpheous.

\section{$1.1 \quad$ Introduction}

The first industrial revolution was driven by the implementation of machinery in the manufacturing chain process at the end of the XVIII century, principally in the textile production. This was mainly due to the fact that engineers of that time could master the steam powered technology.

In the XX century, another leap forward in the manufacturing process occurred with the implementation of industrial robots, particularly devoted to repetitive and non-stoping tasks, during the '60s. The maturity of this technology reached its crest in the '90s, though given their prohibitive cost they were mainly constrained to the industrial environment.

Nowadays, robot applications had seen their way into other environments interacting not only with technicians and highly trained personal but with regular persons as well. Robots have become a more present actor in daily live activities and they are no 


\section{MOTIVATION AND AIMS}

longer a science fiction promise but a reality. This is mainly caused by the evolution of technology in general, providing more powerful computers, new materials and more accurate sensors that are at hand for any enthusiast, in contrast with the situation thirty years ago.

As a consequence, the great challenge in robotics today is to integrate all the appropriate elements and coordinate them in order to performe a specific task, or what is to say to control them.

Control algorithms have been favored with the increase of computing power in todays hardware, given place to the implementation of complex mathematical formulation and the processing of information coming from several sources in real time that were unthinkable a few decades ago.

In particular, control algorithms become complex when the dynamics of the system is highly coupled and its performance must be kept under certain boundaries for a wide working range.

Parallel mechanisms are clearly within this framework. The inherent closed-chain topology make all their elements dependent of each other providing advantages and drawbacks comparing with their serial counterparts.

On the other hand, to the dynamics of the system, the physical properties and limitations of the actuators must be taken into account when designing the a high performance controller.

Based on these considerations, the present work is developed.

\subsection{Aims of this work}

The hydraulic parallel mechanism under study is an open system for the design and implementation of advance control techniques. Within the framework of this work, the following objectives were achieved:

- Comprehensive analysis and modeling of the hydraulic servo actuator.

- Experimental model identification and validation of the hydraulic servo actuator.

- Experimental identification of the parameters of the Lu-Gre friction model.

- Robustness analysis of classical controllers for the hydraulic servo actuator. 
- Design and implementation of adaptive controller for the hydraulic servo actuator.

- Kinematic Analysis of the Parallel Mechanism.

- Dynamic Modeling of the Parallel Mechanism.

- Complete dynamic model implemented in ADAMS.

- Implementation of control strategy based on the dynamic model of the Parallel Mechanism.

\subsection{Contributions of this work}

In this work it has been addressed several topics in the field of modeling, identification and control, whose main contributions are listed below:

- Modeling and analysis of the hydraulic servo actuator. The model of the hydraulic servo actuator consists on two parts. In general, it is only considered the first part that relates the input/output variables. It was demonstrated that not considering the second part also, may lead to erroneous controller design.

- Development of a methodology for the experimental Identification of the Hydraulic servo actuator. The methodology combines LSQR and an ARMAX model.

- PI and PID controller robust stability analysis. It is demonstrated using the small gain theory that the PI controller is robust and that PID and PD controller may lead to instability by observing the well-possedness of the system. Theoretical analysis is verified experimentally.

- Parametric uncertainty of the model vs identified parameter variability.

- Analysis of velocity and acceleration observers.

- Analysis of the performance of the Direct Kinematics numerical approach.

- Modification of the dynamic model of the Parallel Manipulator using virtual work principle considering center of mass of the links outside the axis of action of the prismatic actuator.

- Implementation of the dynamic model of the PM within the control loop. 


\section{MOTIVATION AND AIMS}

At the moment of writing this document, the contributions of this work had resulted in the following published works in order of appearance:

- I.A. Banfield Chamorro, R.J. Saltaren, L.J. Puglisi, C.Garcia Cena, I. Pena Regueiro, R. Aracil, M.N. Cardona, Design of a Hydraulic 6UPS Mechanism for Experimental Research, International Conference CONCAPAN XXXI, IEEE, El Salvador, 2011.

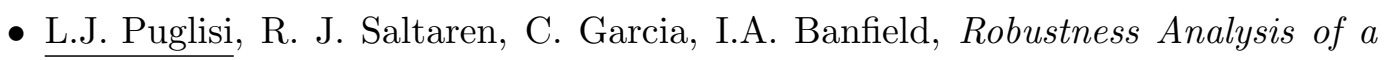
PI Controller for a Hydraulic Actuator, Control Engineering Practice, vol 43, pp 94-108, 2015. DOI: 10.1016/j.conengprac.2015.06.010.

- L.J. Puglisi, R.J. Saltaren, C.E. Garcia Cena, On the Velocity and Acceleration Estimation from Discrete Time-Position Sensors, Control Engineering and Applied Informatics, Vol. 17, N.3, pp. 30-40, 2015.

Also, during the $\mathrm{PhD}$ program it was published:

- H.A. Moreno, R. Saltarn, L.J. Puglisi, I. Carrera, P. Cardenas, C. Alvarez, Robtica Submarina: Conceptos, Elementos, Modelado y Control, Revista Iberoamericana de Automtica e Informtica industrial. Volumen 11, Nmero 01. Enero 2014Marzo2014. Pginas 3?19, Editorial Elsevier, DOI: 10.1016/j.riai.2013.11.001.

- C. Garcia Cena, P. F. Cardenas, R. Saltaren Pazmio, L.J. Puglisi, R. Aracil Santonja, A Cooperative Multi-Agent Robotics System: Design and Modelling, Expert Systems with Applications (2013), Editorial Elsevier, Volume 40, Issue 12, 15 September 2013, Pages 4737?4748. DOI: 10.1016/j.eswa.2013.01.048.

- L.J. Puglisi, R.J. Saltaren, G. Rey Prtoles, H. Moreno, P.F. Crdenas, C. Garcia, Design and Kinematic Analysis of 3PSS-1S Wrist for Needle Insertion Guidance, Robotics and Autonomous Systems (2013), Elsevier Editorial, Volume 61, Issue 5, May 2013, Pages 417-427, DOI: 10.1016/j.robot.2013.02.001.

- L.J. Puglisi, R.J. Saltaren, H. Moreno, P.F. Crdenas, C. Garcia, R. Aracil, Dimensional synthesis of a spherical parallel manipulator based on the evaluation of global performance indexes, Robotics and Autonomous Systems (2012), Volume 60, Issue 8, August 2012, Pages 1037?1045, DOI:10.1016/j.robot.2012.05.013. 
- H. A. Moreno, R. Saltaren, I. Carrera, L.J. Puglisi and R. Aracil, ndices de Desempeo de Robots Manipuladores: una Revisin del Estado del Arte, Revista Iberoamericana de Automtica e Informtica Industrial, Volume 9, Issue 02, AprilJune 2012, Pages 111-122. DOI: 10.1016/j.riai.2012.02.005.

- J.R.Serracin, L.J. Puglisi, R. Saltaren, G. Ejarque, J. M. Sabater-Navarro, R.Aracil, Kinematic Analysis of a Novel 2 DoF Orientation Device, Robotics and Autonomous Systems, Elsevier Editorial, Vol. 60, Issue 6, June 2012, pp. 852-861, DOI: 10.1016/j.robot.2012.01.010.

- C. Garcia, P. Cardenas, L.J. Puglisi, R. Saltaren, Design And Modeling Of The Multi Agent Robotic System: Smart, Robotics and Autonomous Systems, Elsevier Editorial, Vol. 60, Issue 2, Feb. 2012, pp. 143-153. DOI: 10.1016/j.robot.2011.09.007.

- I. Carrera, H. Moreno, R. Saltaren, C. Perez, L.J. Puglisi, C. Garcia, ROAD: Domestic Assistant and Rehabilitation Robot, Medical \& Biological Engineering \& Computing (2011), Volume 49, Issue 10, October 2011, Pages 1201-1211. DOI: 10.1007/s11517-011-0805-4.

\subsection{Structure of this Document}

The work presented in this book is divided into twelve chapter grouped in three main parts.

The first part is completely devoted to the main theoretical aspects treated in this works. In this part is addressed the main concepts related to hydraulic actuation systems, parallel robots and advanced control technique.

Therefore, in this chapter is briefly introduced the main topics concerning this work. It is not the intention to provide a full and detailed treatment of each of them, but instead it should be think of as a first look into their theoretical basis remarking the main literature followed for each of them.

The second part is focused to the modeling, identification and control of the hydraulic servo-actuator. This part can be also thought as a methodology to understand and control any linear hydraulic actuator.

The last part deals with the modeling (kinematics and dynamics) and control of a parallel manipulator, which is powered by a hydraulic servo-actuator. 
Each chapter addresses a specific topic of this research and can be considered individually as a regular paper, which introduce the purposes, the main contributors of that particular field of research and the contribution of this work. 


\section{Part I}

\section{Basic Concepts}





\section{2}

\section{Parallel Mechanism}

"... the world is changed...I feel it in the water, I feel it in the earth, I smell it in the air, Much that once was is lost, for none live to remember it ..."

Lady Galadriel

\section{$2.1 \quad$ Introduction}

In this section is briefly introduced the main concepts regarding parallel mechanism, including a bit of history and some of the most remarkable applications.

\subsection{Parallel Robots}

The mechanical architecture of Parallel Robots (PR) is based on Parallel Mechanisms $(\mathrm{PM})$ in which a member called a moving platform is connected to a reference member by at least two kinematic chains (coined limbs or legs) (see Fig. 2.1). The robot actuators are integrated in the limbs usually closed to the fixed member, also called the base or the fixed platform. The moving platform positions the end-effector in space and may have anything between two and six degrees of freedom. Usually, the number of actuators coincides with the degrees of freedom of the mobile platform, exceeding them only in the case of redundantly-actuated PR (57). 


\section{PARALLEL MECHANISM}

Therefore, a PM can be defined as follows (81):

A parallel mechanism is a multi-degree-of-freedom (multi-DOF) mechanism composed of one moving platform and one base connected by at least two kinematic chains in parallel.

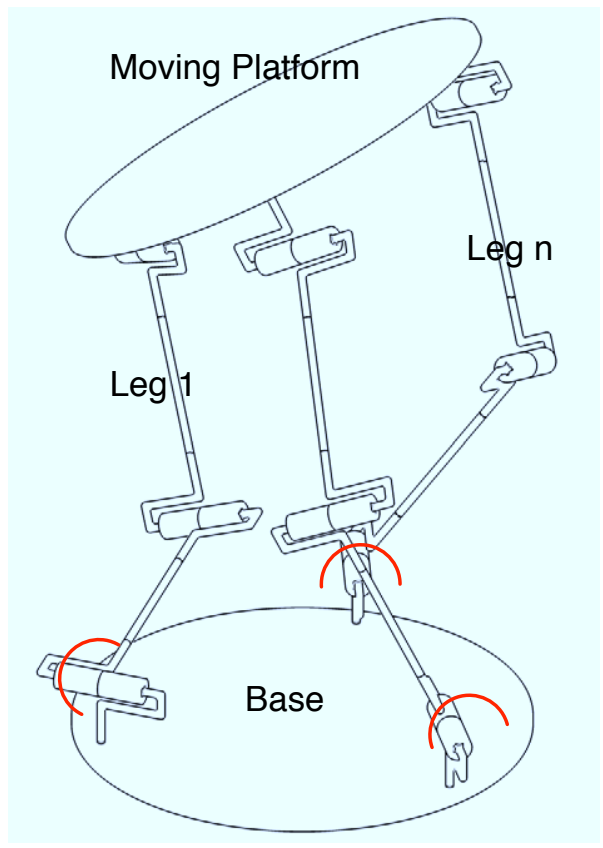

Figure 2.1: Schematic diagram of a general PM.

This structural composition for a mechanism provides several improvement with respect to serial structures. First of all, given that the moving platform (i.e. the end effector) is connected by several legs, the stiffness and rigidity of PM is higher than their serial counterpart. On the other hand, in a PM, the external load is distributed along each leg. Therefore, for a given external load, a PM needs smaller actuator than in serial robot. From another point of view, for a given actuator, the end effector (EF) could reach higher velocity and the mechanism would have higher load capabilities if the mechanism posses a parallel structure.

However, this arrangement of several legs connected to the end effector, is also responsible of the main drawbacks of PM: their reduced workspace. This result is intuitive since from one hand the workspace of the PM will be given by the intersection of the individual workspace of each leg, and from the other hand the possibility of 
collision between their elements increases. There is also another problem associated to $\mathrm{PM}$, and it is the existence of the singular configuration within the workspace, which is also caused by the parallel disposition of kinematic chains and their interaction.

\subsection{Historical Review}

Despite the fact that the implementation of parallel robot has increased considerably in the last few decades, and at certain point it may be considered as new for non-roboticist engineers (indeed, the first book on parallel robot has just appeared in 2000 from the hand of Prof. J.P. Merlet), some of its geometric problem were already introduced during the $X I X$-th century.

However, the concept of the most known parallel robot: the variable length struct octahedral hexapod takes us back to the 50's and 60's with the individually and yet similar contribution of Dr. V.E Gough, D. Stewart and K. Cappel.

Dr. V.E. Gough, inspired by the traditional Multi-Axis Simulation Table (MAST) hexapod (three vertical jacks and three horizontal jacks), rearranged the jacks with an octahedral disposition giving birth to his famous tire-testing machine for analyzing the problems of aero-landing loads, in 1954 at Dunlop Rubber Co.(see Fig. 2.2(a) ).

Nevertheless, it was D. Stewart, who introduced in 1965 the application of an hexapod as a flight simulator. The original concept consisted of three legs composed by two jacks in triangular configuration $2.2(\mathrm{~b})$, but he also suggested the possibility of joining the ends of the jacks at a point linked to the platform, thus reproducing the idea of Gough.

Surprisingly, the first patent of the octahedral hexapod as a motion simulator was filed in 1964 by K. Cappel (see Fig. 2.2(c) ), whose design pursued the improvement of an existing 6DoF MAST machine resulting in the same octahedral arrangement of Dr. Gough.

Even though it can be found records of parallel robots previous to these three examples (see (30) for the disclosure of the history of PM), there is no question that the contribution of these exceptional engineers are the foundational stone for the era of parallel robots. 


\section{PARALLEL MECHANISM}

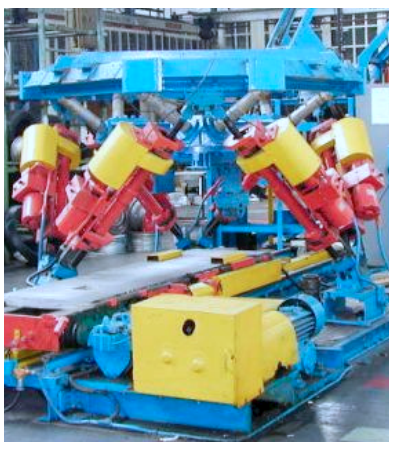

(a)

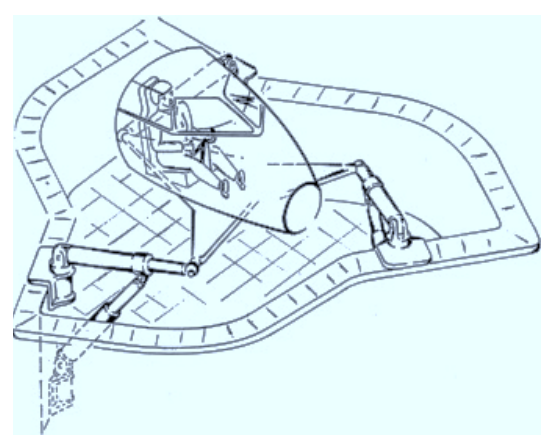

(b)

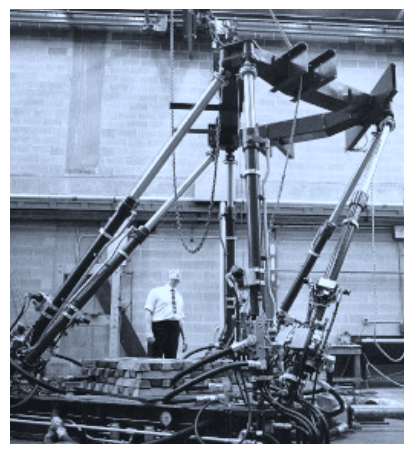

(c)

Figure 2.2: Some of the first implementation of parallel robots. 2.2(a): the tire-test machine of Gough. 2.2(b): the flight simulator concept of Stewart. 2.2(c): the flight simulator proposed by Clavel.

\subsection{Parallel Robots and their applications}

Currently PRs have seen their way into industry, specially for high speed pick and place tasks in mounting lines (see Fig. 2.3(b)). The preferable mechanism for this type of task is the Delta PM first proposed by Prof. R. Clavel (see Fig. 2.3(a) (43)) and their variations. In fact, most of the traditional robot manufacturer offers a version of this PM in their catalogues, such as the ABB IRB 360, FANUC M3iA, YASKAWA MPP3, Adept Quattro series, Delta Series from Asyril, Tosy TI P304-01 among others.

Even though the dynamic properties of the Delta mechanism makes it more than suitable for pick and place tasks, there are many other commercial solutions with this mechanism. One of its main usage is as haptic device, such as the omega3 from force dimension(c) mainly for high-precision medical interfaces purposes (see Fig. 2.4(a)),

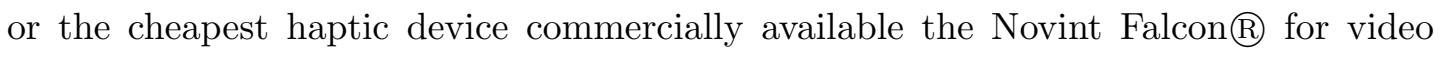
game control. The increasing sector of low cost 3D printer development also presents alternatives based on the Delta mechanism with linear actuator, such as the Rostock MAX and the DeltaMaker ${ }^{\mathrm{TM}}$ (see Fig. 2.4(b)).

On the other hand, when the task requires 6 DoFs the mechanisms based on hexapode are mostly preferred. Traditional robot manufacturers do not provide as many alternative as the Delta robot, indeed the FANUC F-200iB designed for manipulation and assemblage seems to be their only offer (see Fig 2.5(a)). However, there exists consid- 


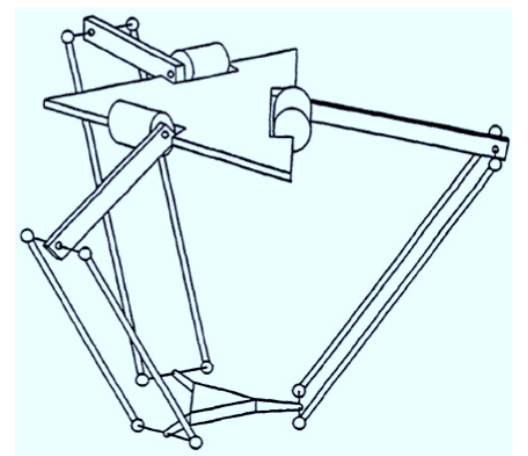

(a)

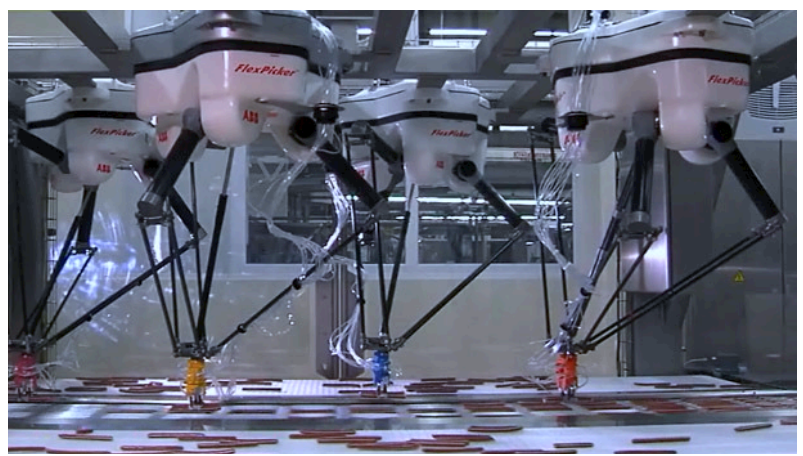

(b)

Figure 2.3: Delta PM. 2.3(a): schematic diagram of the Delta PM from Clavel PhD thesis. 2.3(b): implementation of four Delta PM in a mounting line for sausage packaging (courtesy of ABB robotics).

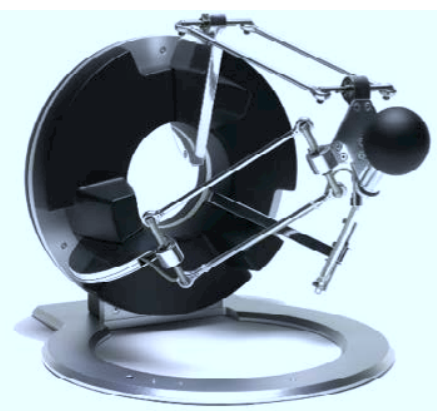

(a)

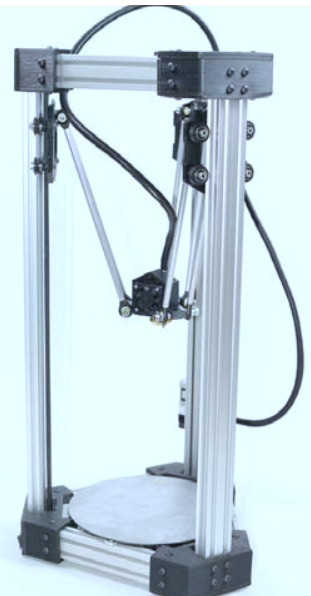

(b)

Figure 2.4: Comertial solutions using Delta robots. 2.4(a): omega3 haptic interfase. 2.4(b): DeltaMaker ${ }^{\mathrm{TM}} 3 \mathrm{D}$ printer.

erably new robot manufacturers that are specialized in PMs, such as PI (specialized in nano positioning, see Fig. 2.5(b)), Mikrolar Inc. (presenting an interesting solution for $360^{\circ}$ see Fig. 2.5(c))rotation, Symétrie and Quaser, among others that cover this spot of the market left by traditional robot manufacturers.

In particular the $6 \mathrm{DoF}$ hexapods has been used in many applications of the most 


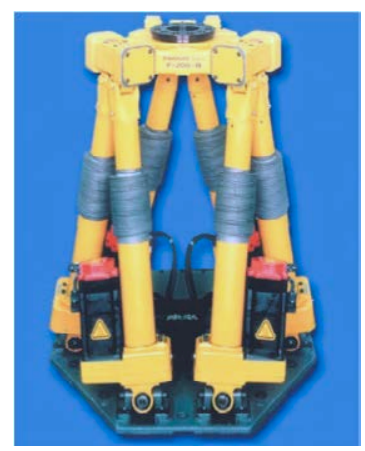

(a)

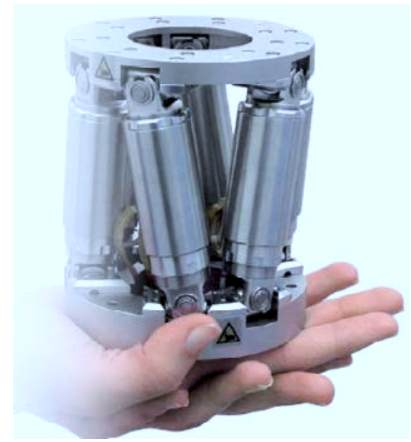

(b)

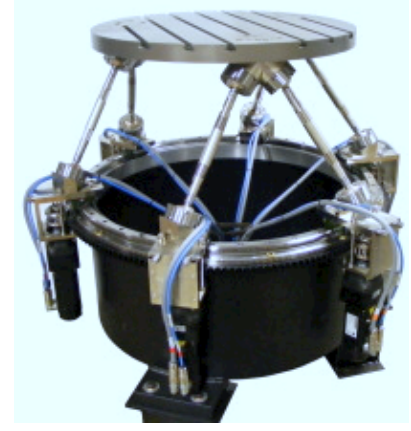

(c)

Figure 2.5: Commercial hexapods. 2.5(a):Fanuc F200iB, 2.5(b): H-810 from PI, 2.5(c): R-3000 Rotopod from Mikrolar Inc.

diverse nature. Continuing with its most known application as motion simulator, it was developed a car driving simulator based on an hexapod embedded on linear rails in order to provide real driving sensation (see Fig. 2.6(a)). Another application that exploits the capabilities of generates $6 \mathrm{DoF}$ motions has seen their way into the special effects industry, such as the hexapod implemented in the PI's Life motion picture as a tide simulator (see Fig. 2.6(b)). Another interesting application is the equestrian gait simulator proposed in [AMIRAT 19996].

The high rigidity that an hexapod provides made it suitable for the torso the Ape Robot created as a demonstrator of the iStruct project developed by the Robotic Innovation Center of the University of Bremen, aiming to improve the efficiency of walking machines by the use of intelligent structures (see Fig. 2.6(c)).

The efforts and ambitious project of the ESO (European Southern Observatory) carried on at the Atacama's desert at the north of Chile, implements hexapods in their telescopes in order to provide precise and accurate positioning of the active mirrors (see Fig. 2.6(d)). Currently, the active optics of the ALMA (Atacama Large Millimeter/submillimeter Array) deployment uses PI's nano-positioning hexapods.

On the other hand, a $6 \mathrm{DoF}$ hexapod can also be seen as a variable geometry structure active device. This characteristic is the fundamental stone of the REMO project developed by the Group of Robots and Intelligent Machine of the Universidad Politécnica de Madrid, where an hexapod is implemented as an underwater vehicle with vectorized 
thrusting providing high maneuverability (131), (16), (154), (105).

Another project that benefits of the variable geometry capabilities of an hexapod is the TREPA. In this project, an hexapod robot climber was first designed for climbing palm trees at the University of Elche (9), (8), and further enhanced for its used in inspection and maintenance of tubular structures (132), (12), (133), (21).

Others application of hexapods are rehabilitation trainnig (27), haptic devices (127), active Helideck for floating structure (36), minimally invasive surgery (137), among others.

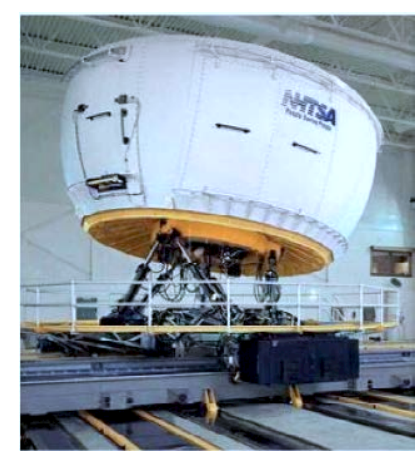

(a)

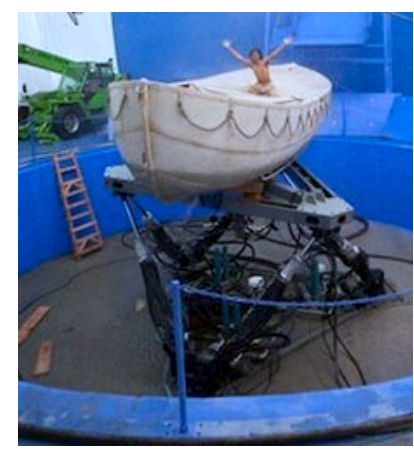

(b)

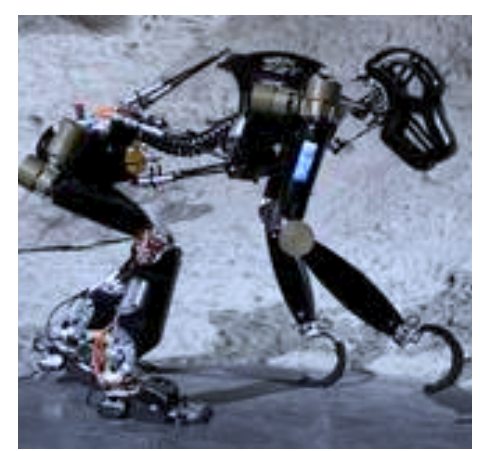

(c)

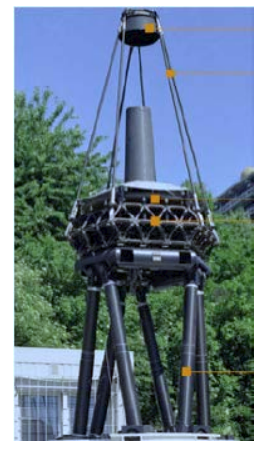

(d)

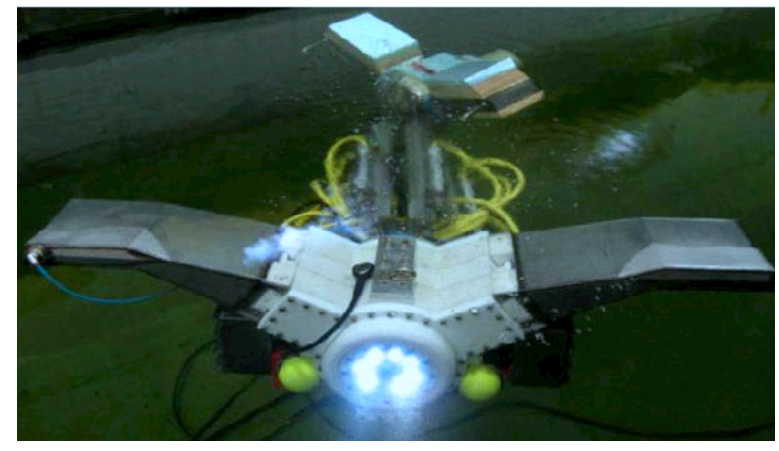

(e)

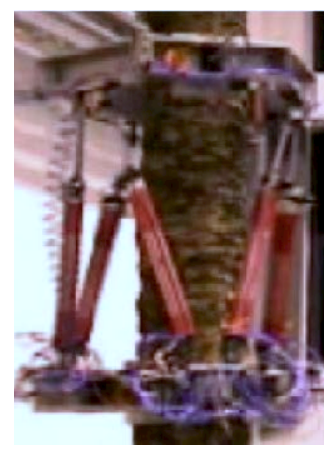

(f)

Figure 2.6: Applications of hexampods PM. 2.6(a): Driving simulator, 2.6(c): the Ape Robot from the iStruct project,2.6(b):tide simulations from PI's Life motion picture special effects, 2.6(d): details of the subreflector mechanism of the telescopes at Atacama, 2.6(e): the underwater vehicle REMO I, 2.6(f): the climbing robot TREPA.

Other PMs commonly used in applications are those topologies that provide spherical motions. Perhaps, the most known spherical parallel mechanism (SPM) is the Agile 


\section{PARALLEL MECHANISM}

Eye (60), a $3 \underline{R R R ~ P M ~ d e v e l o p e d ~ a t ~ t h e ~ L a v a l ~ U n i v e r s i t y ~ f o r ~ i t s ~ i m p l e m e n t a t i o n ~ f o r ~ f a s t ~}$ camera orienting (see Fig.2.7(a)).

The interest of developing SPMs is to provide accuracy and rigidity in the orientation. These characteristics fulfill the requirements of surgical applications, such as the implementation of a 3UPS-1S PM for minimally invasive surgery (109), or the 2UPU1U PM for maxilo-facial intervention (135). This type of mechanism can be combined with serial structure providing an hybrid robotic with large workspace and accurate positioning as the hybrid robot presented in (130) (122) for needle insertion surgery porposes, where a SPM is coupled with a traditional $3 \underline{\mathrm{R}}$ serial robot (see Fig. 2.7(c)). Another example of the combination of serial and parallel topology is the robot for the assembly and maintenance of the ITER. This hybrid robot has ten DOFs and consists of a carriage which provides fours DOFs that enlarge the workspace to offer high mobility and the 6UPS that support the end effector and tool [AILSAEDI 2014].

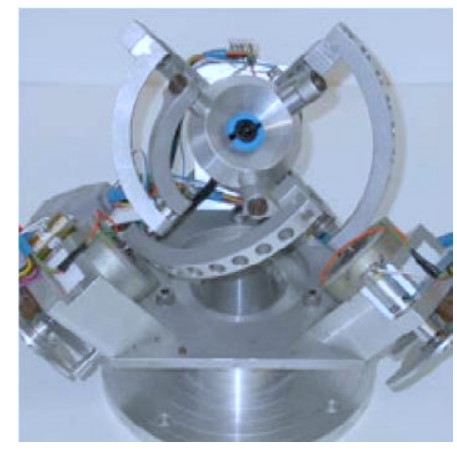

(a)

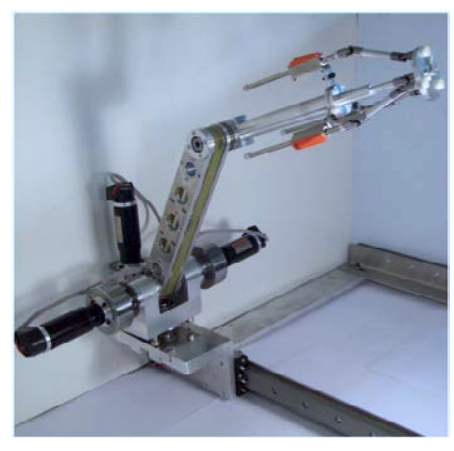

(b)

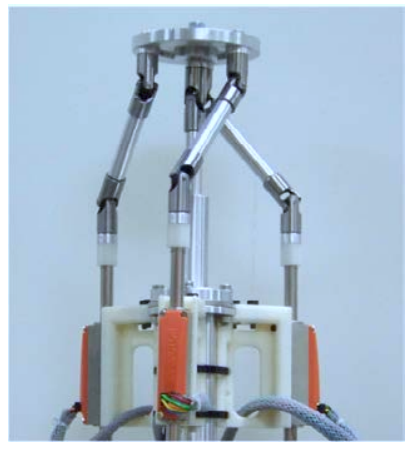

(c)

Figure 2.7: Spherical Parallel Mechanism. 2.7(a): Agile Eye, 2.7(b): hybrid kinematic robot, 2.7(c): 3ㅍSS-1S SPM.

Other topologies and their applications are presented and discussed in chapter two of (98), were an extensive review of parallel robots (until 2006) is introduced.

\subsection{Kinematic Formulation}

Kinematics deals with the aspects of motion without regard to the forces and/or torques that cause it. 
The kinematic modeling of a robot consists on the determination of the relations of the operational coordinates of the robot $\left(\boldsymbol{x} \in \mathbb{R}^{n}\right.$, where $n$ is the dimension of the operational space) and its joints variables $\left(\boldsymbol{q} \in \mathbb{R}^{m}\right.$, where $m$ is the number or joints of the mechanism).

In particular, given the nature of the closed kinematic chains in PMs, this relationship is constrained to:

$$
\boldsymbol{f}(\boldsymbol{x}, \boldsymbol{q})=\mathbf{0}
$$

From the analytical point of view, there are commonly two main aspects of the kinematics modeling: Position Modeling and Velocity Modeling.

\subsubsection{Position Modeling}

It establishes the geometric relation between the coordinates $\boldsymbol{x}$ and $\boldsymbol{q}$. Depending which coordinates are known and which are unknown, there are two main formulations, the inverse formulation: $\boldsymbol{q}=\boldsymbol{g}^{-1}(\boldsymbol{x})$, and the direct formulation: $\boldsymbol{x}=\boldsymbol{g}(\boldsymbol{q})$.

In general, the first one can obtained from simple observation resulting in simple geometric relations. On the other hand, the direct formulation are complex polynomial equations that rarely provide an unique solution and sometimes there is no a solution at all (144) (100). For instance, the solution of the DK of a 3 DoFs planar mechanism may have up to 6 different poses depending on the topology of the mechanism (97),(119). The spherical $3 \underline{R} R R$ posses four different assembly modes (31), and the special offset3U $\underline{P} U$ translational PM has 16 possible solution (74). If the PM has more DoFs the problem is even worse: the decahedral PM has at least 48 different solutions (75), the 5-RPUR PM (5 DoFs) has 208 real solutions (95), and Stewart-Gough-type platforms (6 DoFs) has 40 possible solutions (124), (50), that under certain geometrical conditions they can be reduced to 16 different poses (108). In order to reduce complexity of the polynomial equations, it is sometime implemented redundant sensors (13),(32).

On the other hand, numerical methods appear like a strong alternative to find the solution of DK. In general these methods rely on a search algorithm governed by an optimization criteria. Many authors use neural network (33), (86), (118), genetic algorithm (39), (148), (34), (115), Newton-Raphson (NR) (52), (141), Taylor series (129), fuzzy logic (71) or interval analysis (96). 


\section{PARALLEL MECHANISM}

\subsubsection{Instantaneous or Velocity Modeling}

It establishes the relation between the velocity of the end effector coordinates $\dot{\boldsymbol{x}}$, and the joints velocity vector coordinates $\dot{\boldsymbol{q}}$, given by:

$$
\begin{aligned}
\frac{\partial}{\partial \boldsymbol{x}} \boldsymbol{f}(\boldsymbol{x}, \boldsymbol{q})+\frac{\partial}{\partial \boldsymbol{q}} \boldsymbol{f}(\boldsymbol{x}, \boldsymbol{q}) & =0 \\
\boldsymbol{J}_{\boldsymbol{x}} \dot{\boldsymbol{x}} & =\boldsymbol{J}_{\boldsymbol{q}} \dot{\boldsymbol{q}} \\
\dot{\boldsymbol{x}} & =\boldsymbol{J} \dot{\boldsymbol{q}},
\end{aligned}
$$

where $\boldsymbol{J}_{\boldsymbol{x}}$ is the direct Jacobian matrix, $\boldsymbol{J}_{\boldsymbol{q}}$ is the inverse Jacobian matrix, and $\boldsymbol{J}=$ $\boldsymbol{J}_{\boldsymbol{x}}{ }^{-1} \boldsymbol{J}_{\boldsymbol{q}}$ is the Jacobian matrix of the robot (whenever $\boldsymbol{J}_{\boldsymbol{x}}{ }^{-1}$ exists).

In order to obtain this relationship there exist two techniques. The first approach is the classical method based on the differentiation of the inverse kinematic model. The second, uses the concepts of the screw theory. Both techniques leads to the same results, however the last one provides a better insight of the geometry and physics of the mechanism.

\subsubsection{Jacobian Matrix Based on Screw Theory}

Considering a serial kinematic chain which is composed by $l$ joints, the resulting instantaneous twist at the end effector $\boldsymbol{\$}_{p}=\left[\boldsymbol{w}_{n}, \boldsymbol{v}_{o}\right]$ can be found as the combination of individual instantaneous twists $\$_{i}$ associated to each joint as follows (145):

$$
\boldsymbol{\$}_{p}=\sum_{j=i}^{l} \dot{q}_{i} \hat{\boldsymbol{\$}}_{i}
$$

where $\dot{q}_{i}$ is the intensity of the instantaneous unitary twist $\hat{\$}_{i}, \boldsymbol{w}_{n}$ is the angular velocity and $\boldsymbol{v}_{o}$ is the linear velocity of a point that is instantaneously coincident with the origin.

A parallel manipulator has $m$ limbs, therefore the resulting instantaneous twist at the end effector $\$_{p}$ can be found as:

$$
\boldsymbol{\$}_{p}=\sum_{j=i}^{l} \dot{q}_{i, j} \hat{\$}_{i, j}, \quad \text { for } j=1,2, \cdots, m
$$

However, a parallel manipulator has many unactuated joints, that can be eliminated from (2.4), by applying the theory of reciprocal screws. This concept was first studied 
by Ball (20), and it states that two screws, $\$_{1}$ and $\$_{2}$, are said to be reciprocal if they satisfy the following condition (81):

$$
\$_{1} \circ \$_{2}=\left[\Pi \$_{1}\right]^{T} \$_{2}=0
$$

where

$$
\boldsymbol{\Pi}=\left[\begin{array}{cc}
\mathbf{0} & \boldsymbol{I}_{3} \\
\boldsymbol{I}_{3} & \mathbf{0}
\end{array}\right]
$$

where $\boldsymbol{I}_{3}$ is the $3 \times 3$ identity matrix and $\mathbf{0}$ is the $3 \times 3$ zero matrix. The operator $\circ$ is defined as the reciprocal product of two screws.

In particular, if a wrench (i.e. a system of forces) represented as a screw acts on a body in such a way that it produces no work while the body is undergoing an infinitesimal twist (i.e. an instantaneous motion) represented as a screw, the two screws are said to be reciprocal and its virtual work is null.

Assuming that there exists a reciprocal screw $\$_{r, i}$ for each limb which is reciprocal for all the unactuated joint of that limb and implementing the reciprocal product to (2.4), it results in:

$$
\begin{gathered}
{\left[\begin{array}{c}
\$_{r, 1} \\
\boldsymbol{\$}_{r, 2} \\
\vdots \\
\boldsymbol{\$}_{r, m}
\end{array}\right] \boldsymbol{\$}_{p}=\left[\begin{array}{c}
\boldsymbol{\$}_{r, 1} \circ \hat{\boldsymbol{\$}}_{i, 1} \\
\boldsymbol{\$}_{r, 2} \circ \hat{\boldsymbol{\$}}_{i, 2} \\
\vdots \\
\boldsymbol{\$}_{r, m} \circ \hat{\boldsymbol{\$}}_{i, m}
\end{array}\right] \dot{\boldsymbol{q}}} \\
\boldsymbol{J}_{\boldsymbol{x}} \boldsymbol{\$}_{p}=\boldsymbol{J}_{\boldsymbol{q}} \dot{\boldsymbol{q}},
\end{gathered}
$$

where $\hat{\$}_{i, j}$ is the unitary instantaneous twist associated to the actuated $i-t h$ joint of the $j-t h$ limb.

\subsection{Kinematics Analysis}

The kinematic analysis of PM comprises the study and analysis of the workspace, presence and identification of singular configurations and dexterity of the mechanism.

The workspace of a mechanism is defined as all the possible configurations that it can achieve with out exceeding its geometrical and physical limitations. The geometrical limitation are imposed by the topology of the mechanism, while the physical limitation are given by the range of work of its joints and the collision between its elements. 


\section{PARALLEL MECHANISM}

Unfortunately, the analytical determination of the workspace of a PM is impossible, therefore a try-error approach is the most implemented practice. This approach defines an objective workspace and discretizes it in several space configurations $\boldsymbol{x}$. Then, by means of the inverse position modeling, it is verified whether a particular configuration belongs to its workspace or not, by analyzing the range of work of all the joints and the possible collision between the parts of the PM (see Fig. 2.8).

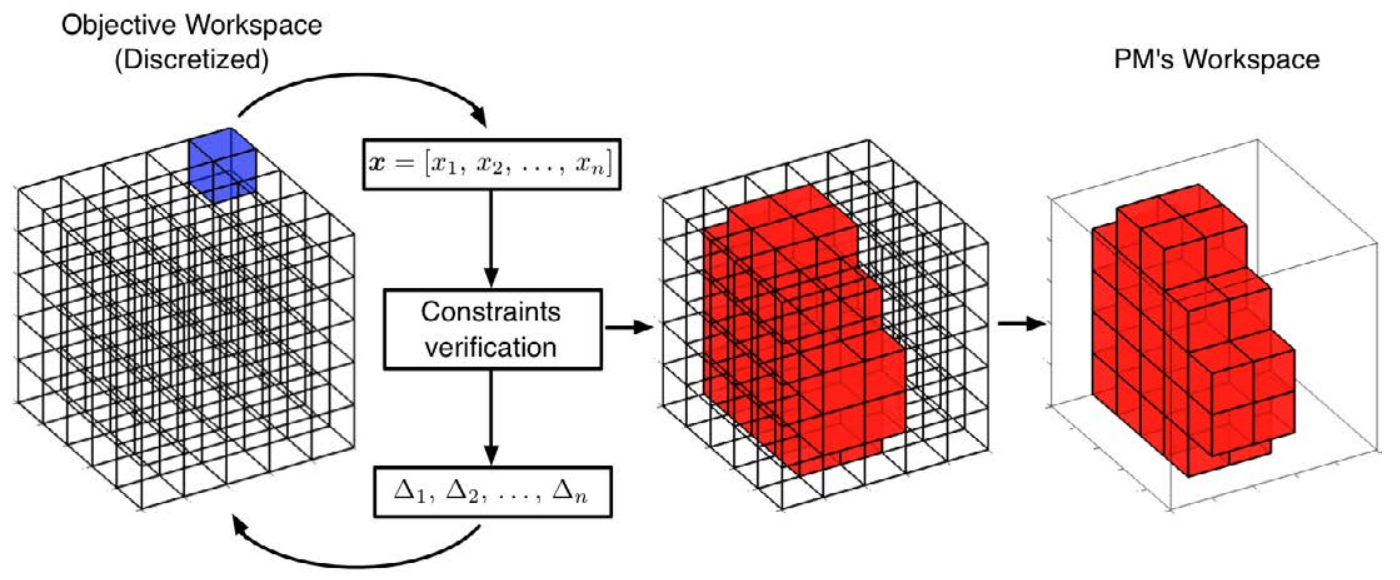

Figure 2.8: PM's workspace determination, where $\boldsymbol{x}$ is the space coordinate vector and $\Delta_{1}, \Delta_{2}, \cdots, \Delta_{n}$ is the discretization parameter associated to each space coordinate.

A singular configurations of a PM is defined as a configuration where the instantaneous kinematic equation $\boldsymbol{J}_{\boldsymbol{x}} \dot{\boldsymbol{x}}=\boldsymbol{J}_{\boldsymbol{q}} \dot{\boldsymbol{q}}$ is not satisfied, which means that either $\boldsymbol{J}_{\boldsymbol{x}}$ or $\boldsymbol{J}_{\boldsymbol{q}}$, or both lose their ranks. In these particular configurations the mechanism may lose or win DoFs, thus turning the mechanism uncontrollable. The analysis of singular configurations is of main importance in PMs since they reduce the effective workspace, resulting in one of the main drawbacks of PMs.

There are two approaches that are commonly implemented for the determination of singular configurations:

1. Numerical. This approach is based on the evaluation of the determinant of the Jacobian matrix, and find the configurations where it is null. Since the probability to find the exact configuration where this situation occurs is infinitesimal, a better approach is to identified where the sign of the determinant changes. 
2. Analysis of the Jacobian Matrix.The Jacobian matrix is given by vectorial operations on elements that are associated with the topology of the PM, such as direction vectors, points of actions, etc. Therefore, it is possible to analyze where the Jacobian matrix looses its ranks by observing the behaviour of these vectors by finding perpendicular, colinear, coplanar patterns.

The dexterity of a PM is related with the capability of transmiting energy from the joint space to the task space (or viceversa), therefore once again the Jacobian matrix of PM has a main role in this analysis. There are many ways to measure this dexterity, in general they are defined in term of performance indexes. The most commonly used indexes in PMs are (94):

- Condition Index (CI): which is given by the inverse of the condition number of the Jacobian matrix for a given configuration.

- Global Condition Index (GCI): defined as:

$$
G C I=\frac{\int_{W} C I(\boldsymbol{J}) d W}{\int_{W} d W},
$$

where $W$ is the PM's workspace, an gives an idea of the performance of the mechanism in all its workspace.

The list of indexes is extent, and in general the indexes are somehow related with the Jacobian matrix. A review of kinetostatic performance indexes can be found in (106). The use of performance indexes as a measure of the dexterity of a PM is not limited to the quantification performance but also they are vastly used for the optimization of the dimensional synthesis of PM (121).

\subsection{Dynamic Modeling}

Dynamics deals with the relations between forces and/or torques applied to a (rigid) body and the motion that they generate. The derivation of this relationship is known as the dynamic model of the rigid body.

The dynamics modeling of rigid bodies presents two aspects:

- Direct Dynamics: it tries to determine the resultant motion of a rigid body (or system of rigid bodies), given a set of forces and/or torques applied to it (or them). 


\section{PARALLEL MECHANISM}

- Inverse Dynamics: it tries to find the needed forces and/or torques to produce a given motion.

The solution of the dynamic problem of a rigid body can be found by two main methodologies:

1. Numerical approach: they are based on recursive calculations and global algorithms that provide solution to almost any mechanical system. Commercial software such as ADAMS and DADS relays in this approach also known as computational dynamics. Even though their performance for real time control is still poor, since they consume too many resources during computation.

2. Algebraic approach: they try to find the dynamic equation of the mechanical system by applying the basic principle of dynamics. The solution must be derived for each mechanism and in general it can not be extended to other mechanism. However, for control purposes this methodology is preferable.

The general algebraic form of the inverse dynamic model of a robot is given by:

$$
\boldsymbol{\tau}=\boldsymbol{M}(\boldsymbol{q}) \ddot{\boldsymbol{q}}+\boldsymbol{C}(\dot{\boldsymbol{q}}, \boldsymbol{q})+\boldsymbol{G}(\boldsymbol{q}),
$$

where $\boldsymbol{q}$ is the joint state coordinate vector, $\boldsymbol{M}(\boldsymbol{q})$ is inertia matrix, $\boldsymbol{C}(\dot{\boldsymbol{q}}, \boldsymbol{q})$ is the Coriolis matrix, and $\boldsymbol{G}(\boldsymbol{q})$ is the gravity matrix.

In particular, the dynamic modeling of a PM brings a level of complexity absent in their serial counterpart. The existence of closed kinematic chains makes necessary a strategy in order to formulate the dynamic equation. The two most used strategies are:

1. Consider all the bodies as individual elements and then apply all of the joint constraints simultaneously.

2. The PM is virtually disassembled into $n$ kinematic open chain (one for each leg) and then apply the loop-closure constraint.

The first strategy results in large and sparse matrix equations. The second one is the best choice for typical closed-loop systems. It allows to extend the formulations used in serial mechanism. Indeed, the individual analysis of each virtual open chain can be performed with classical Newton-Euler or Lagrangian approach. The traditional 
Newton-Euler formulation requires the equations of motion to be written once for each body of a manipulator, which inevitably leads to a large number of equations and results in poor computational efficiency. The Lagrangian formulation eliminates all of the unwanted reaction forces and moments at the outset, and thus its more efficient.

The loop-closure constraint can also be formulated with these classical approach, however, to derive explicit equation of motion in terms of a set o independent generalized coordinates becomes a prohibitive task. To simplify the problem additional coordinates along with the set of Lagrangian multipliers are often introduced.

Besides of the classical approaches to derive the dynamic model, it is also common to use the principle of virtual work, which appears to be the most efficient method of analysis.

\subsubsection{Virtual Work Formulation}

A virtual displacement of a system refers to an infinitesimal change in the configuration of the system as a result of any arbitrary infinitesimal changes of the coordinates that are compatible with the forces and constraints imposed on the system at a given instant in time.

The principle of virtual work states that a system is under equilibrium if and only if the virtual work vanishes for any infinitesimal virtual displacement. This is true if the virtual displacements are compatible with the constraints imposed on the system (145).

\subsubsection{Task Space and Joint Space Dynamics}

The dynamic problem can be formulated either in the joint space or the task space. These two formulations are related by the Jacobian matrix and its time derivative as follows.

The expression 2.2, maps the joint space velocity into the task space velocity, and taking the its time derivation it is found:

$$
\ddot{\boldsymbol{x}}=\dot{\boldsymbol{J}} \dot{\boldsymbol{q}}+\boldsymbol{J} \ddot{\boldsymbol{q}}
$$

On the other hand, the energy consumed by the robot must be the same if its evaluated on the joint space or in the task space, i.e.

$$
\Gamma^{T} \dot{\boldsymbol{x}}=\boldsymbol{\tau}^{T} \dot{\boldsymbol{q}}
$$


equating 2.2 in the previous relation it can be found:

$$
\begin{aligned}
\boldsymbol{\Gamma}^{T} \dot{\boldsymbol{x}} & =\boldsymbol{\tau}^{T} \dot{\boldsymbol{q}}, \\
\boldsymbol{\Gamma}^{T} \boldsymbol{J} \dot{\boldsymbol{q}} & =\boldsymbol{\tau}^{T} \dot{\boldsymbol{q}} \\
\boldsymbol{\Gamma}^{T} \boldsymbol{J} & =\boldsymbol{\tau}^{T}, \\
\boldsymbol{J}^{T} \boldsymbol{\Gamma} & =\boldsymbol{\tau}
\end{aligned}
$$

These expression 2.10 and 2.12 allows to express the dynamic model in the joint space given by 2.9 in the task space:

$$
\boldsymbol{\Gamma}=\boldsymbol{M}_{\Gamma} \ddot{\boldsymbol{x}}+\boldsymbol{C}_{\Gamma}+\boldsymbol{G}_{\Gamma},
$$

where,

$$
\begin{aligned}
\boldsymbol{M}_{\Gamma} & =\left(\boldsymbol{J}^{T}\right)^{-1} \boldsymbol{M} \boldsymbol{J}^{-1} \\
\boldsymbol{C}_{\Gamma} & =\left(\boldsymbol{J}^{T}\right)^{-1}\left(\boldsymbol{C}-\boldsymbol{M} \boldsymbol{J}^{-1} \dot{\boldsymbol{J}} \dot{\boldsymbol{q}}\right) \\
\boldsymbol{G}_{\Gamma} & =\left(\boldsymbol{J}^{T}\right)^{-1} \boldsymbol{G} .
\end{aligned}
$$




\section{3}

\section{Hydraulic Systems}

"you have been given a gift ... with a great power comes a great responsabillity ..."

Ben Parker.

\subsection{Introduction}

Hydraulic actuation systems are very important in modern industry, principally because they can generate very high torques and forces, exhibit rapid responses, have a high power-to-weight ratio, and high stiffness. This conjugation of properties can not be matched by any other current commercial technology.

For the development of this work it was necessary a comprehensive understanding of several concepts related to hydraulic.

Therefore, in this chapter is briefly introduced the main topics and concepts regarding hydraulic system that gives support to the second part of this book.

\subsection{Hydraulic Actuation Systems}

composed by a hydraulic Power station responsible of the circulation of oil fluid along the hydraulic circuit at a given flow and pressure, controlled oriffic

In general, a hydraulic actuation system is mainly composed by a hydraulic power station responsible of the circulation of oil fluid along the hydraulic circuit at a given 


\section{HYDRAULIC SYSTEMS}

flow and pressure; controlled orifices which regulate the flow and a mechanical transducer that transform differential pressure and oil flow into a force or torque (see Fig. 3.1). These main elements can be from different nature and its selection will depend on the application.

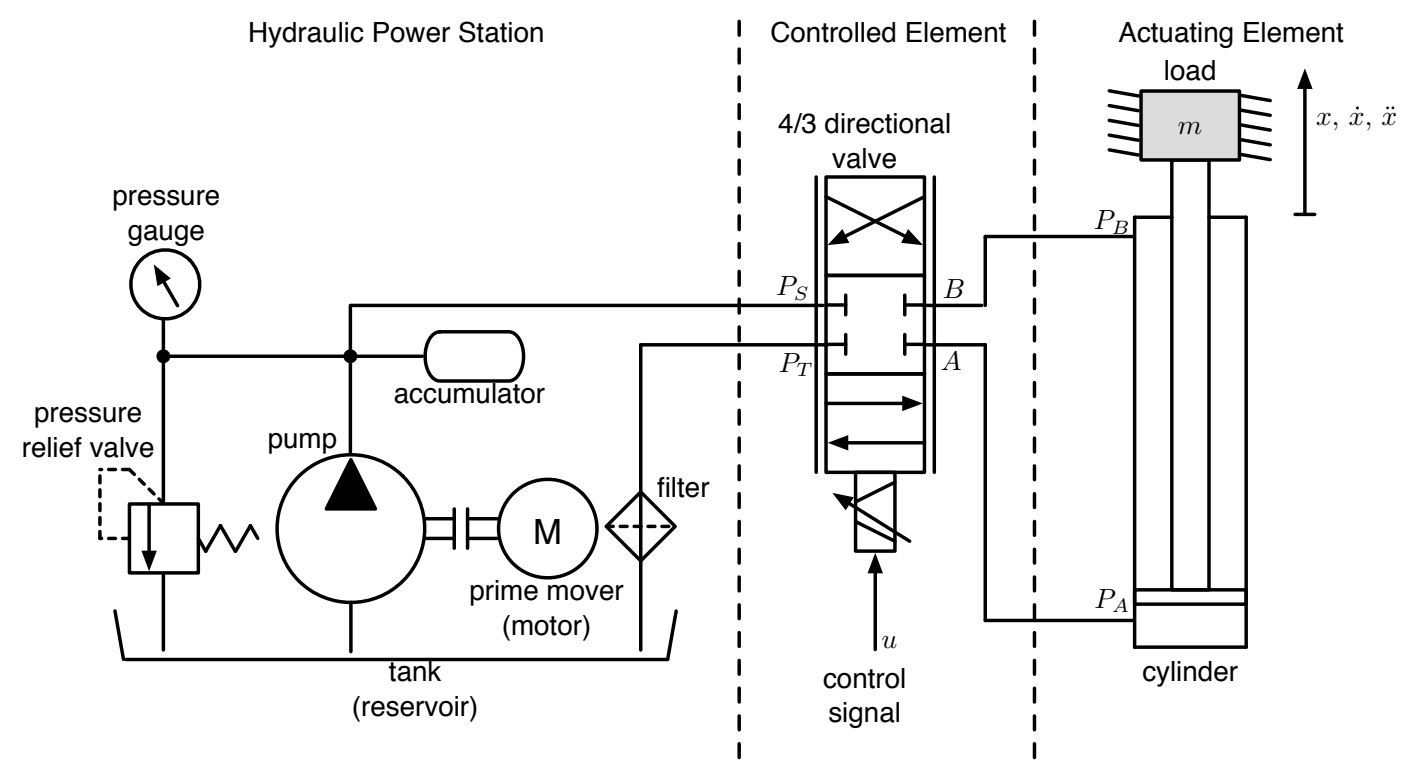

Figure 3.1: Schematic diagram of a general hydraulic system.

In order to provide a controlled force or torque, the control of an hydraulic system can be separated into two different strategies: using a controlled valve or using a controlled power station. In a system with a controlled valve the power efficiency may result poor. However, this scheme permits to use only one power station. The second one, has a very big power efficiency but it requires an individual power station for each actuator, which results very expensive (85).

From the point of view of dynamics and control design, these actuation systems exhibit a significant nonlinear behavior due to the nonlinear flow/pressure characteristics, presence of deadbands in valve operation, oil compressibility, time varying frequency behavior, nonlinear transmission effects, flow and friction forces acting on the spool of the valve, which are not only largely uncertain but also greatly influenced by external load disturbances. Apart from the inherent nonlinearities of the system, there also exists parametric uncertainties which arise from the lack of knowledge of various physical 
parameters of the system, e.g., the payload lifted by an industrial hydraulic manipulator, change in the bulk modulus of the hydraulic fluid due to change in temperature or introduction of foreign particles, etc.

Therefore, for high performance system it is necessary advanced control techniques, which where prohibitive a few decades ago. The complexity of such controllers is becoming more and more feasible given the power and low cost of real time processor available today.

From the point of view of implementation, hydraulic system has two main drawbacks, one is the need of a hydraulic power station, which are in general very heavy, bulky and noisy, and therefore it is not appropriate for portable system, and the other, is that it is considered a dirt technology since its relies on the flow of oil and it is avoided for white-room implementations.

\subsection{Brief Description of Hydraulic Elements}

The main elements of a hydraulic actuation systems is briefly addressed in the following sections. These elements can be from different nature and its selection will depend on the application. For a detailed description of them and their characteristics the reader is referenced to (101), (149), (72).

\subsubsection{Hydraulic Power Station}

The Hydraulic Power Station (HPS) is the power source of the hydraulic system, it is responsible of providing oil flow at a defined flow rate and pressure.

A HPS is mainly composed by a tank (where the oil rest), a hydraulic pump , a filter (to filter the residual particles in the oil) and optionally an accumulator (to filter pressure pulsations from the pump).

The heart of the HPS is the pump, which moves fluid from the tank to the system driven by a mechanical action (i.e. the prime mover, which in general is an electrical motor), thus converting mechanical energy into hydraulic energy.

The pump is an integral part of the control scheme in open loop control, requiring a controlled variable displacement pump. However, in hydraulics servo controls the pump is part of an auxiliary unit that can be considered simply as a source of hydraulic power. 


\section{HYDRAULIC SYSTEMS}

In general, industrial implementations positive displacement or hydrostatic pumps. In this type of pumps an expanding chamber that is initially attached to an inlet port accommodates fluid forced into it by the prevailing fluid pressure at the inlet. The chamber is then mechanically connected to an outlet port, reducing its volume and the fluid is expelled.

Spur Gear pumps are a classical example of this principle. It consists of two identical gears, whose axes are parallel. The fluid is accumulated in the cavity between the teeth of the gear (chamber's expansion, see Fig. 3.2(a)), and carried around its perimeter (see Fig. 3.2(b)- Fig. 3.2(e)). When the teeth of the two gear mesh (chamber's contraction), the fluid is deposited at the outlet port (see Fig. 3.2(f)), and thus the fluid is pumped from the inlet to the outlet port.

These devices are the least expensive of all, and its efficiency is limited. In this arrangement there is significant fluid that is never expelled from the contracting cavity.

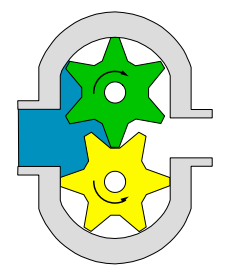

(a)

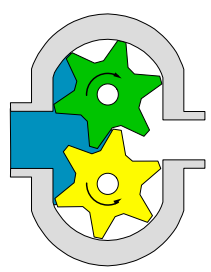

(b)

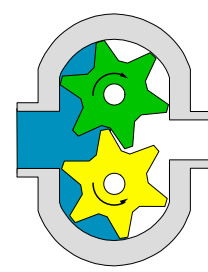

(c)

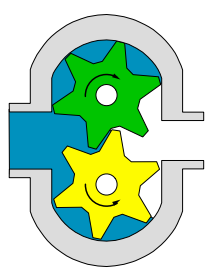

(d)

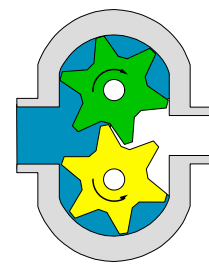

(e)

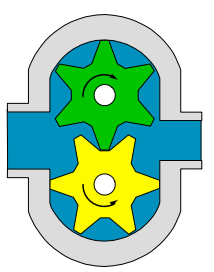

(f)

Figure 3.2: Positive displacement pump concept. Spur gear pump example.

Gear pumps are the most common pumps in industrial environment, however it must be recalled that there exists several approaches to achieve positive displacement. In Fig. ?? it is schematized the tree of the technologies used for PDP, and the reader is addressed to (123) for further details and analysis.

\subsubsection{Valves}

Valves are the most important element in a hydraulic system since they control the amount of power delivered to the actuator and others subsystems. The principle of operation of most valves is the same. Basically, a valve is a variable area orifice by whom the flow passes through. 


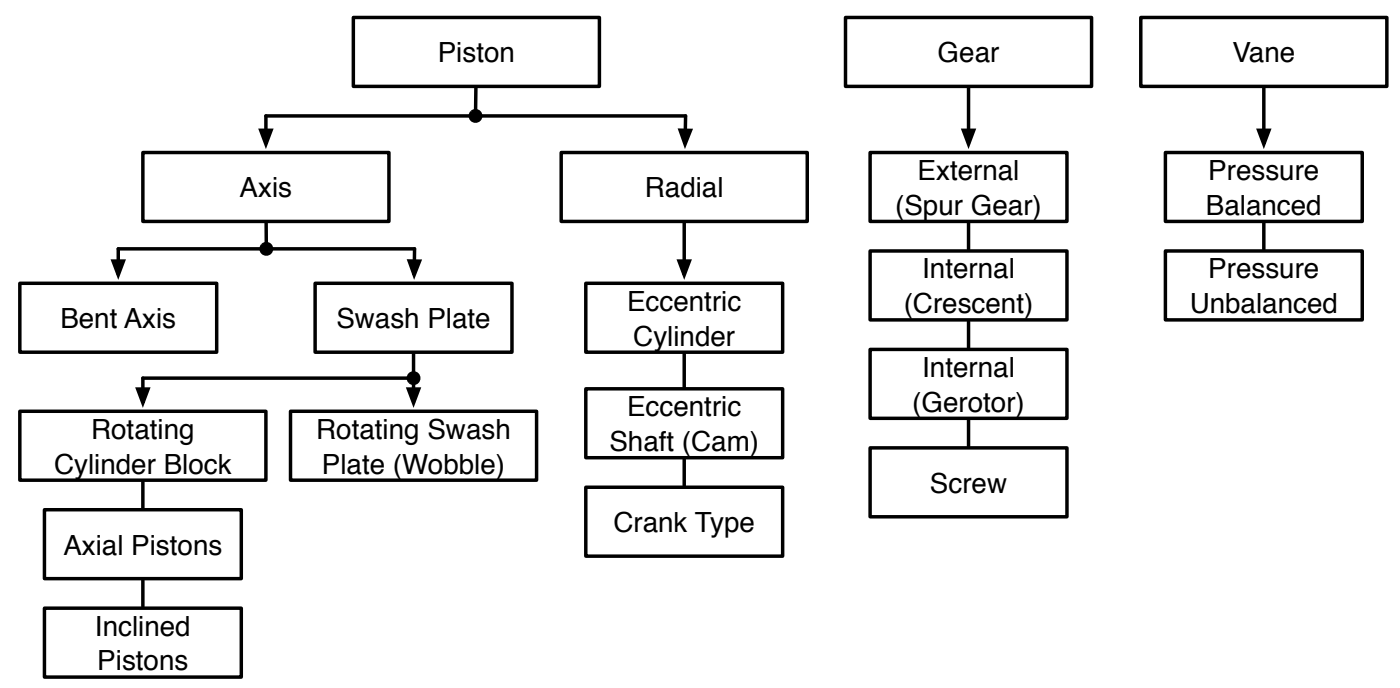

Figure 3.3: Displacement pumps family tree.

The relation between the flow that passes through the orifice and its differential pressure can be derived from the Bernoulli's principle of energy given by:

$$
Q=\alpha_{d} A \sqrt{\frac{2}{\rho}\left(P_{1}-P_{2}\right)},
$$

where $\alpha_{d}$ is the discharge coefficient, $A$ is the surface of the orifice, $\rho$ is the fluid's density, $P_{1}$ and $P_{2}$ are the pressure of the fluid before and after it passes through the orifice (see Fig. ??.

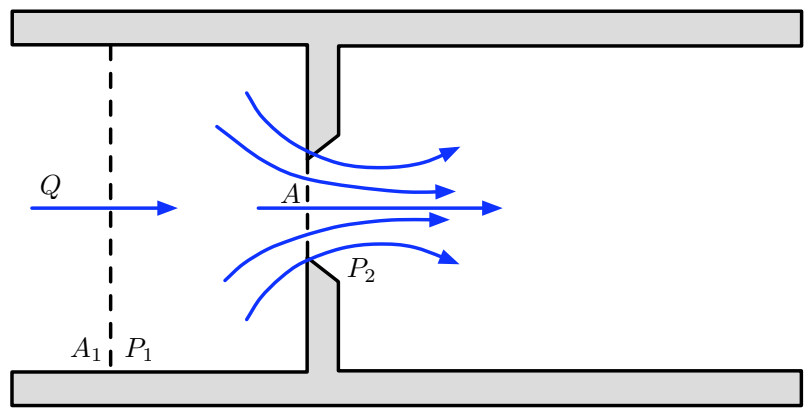

Figure 3.4: Flow through an orifice.

The simplest variant of valves used for fluid power control is the solenoid-valve 3.5 (a), where a controlled element is electrically or mechanically actuated to com- 


\section{HYDRAULIC SYSTEMS}

pletely block or unblock the orifice, turning it into a hydraulic switch. The on/off characteristics of this type of valves limits the performance of controlled fluid power applications.

This limitation was surpassed with the advent of proportional solenoids in 1970s that allows to a sliding element (named spool), to partially blocked the orifice area. Based on this principle, proportional valves can deliver spool position or flow or pressure proportional to the input signal.

On the other hand, servo-valves are high-sophisticated control valves where the position of the spool is controlled by internal electrical and mechanical closed-loop. Servo-valves are commonly classified according to number of stages that it has, number of input/output ports and the number of lands. In general, a single-stage servo-valve comprises a torque motor, which positions the spool via a direct connection. These valves are simple and inexpensive, but have limited flow capacity (up to $20 \mathrm{dm}^{3} / \mathrm{min}$ ) due to the limited power capability of torque motors. Therefore, in order to overcome this limitation one or more hydraulic pre-amplifiers (stages) are needed. These preamplifiers can be flappers, jet pipes, spools, and inclusive another servo-valve. A two stage $4 / 3$ servo-valve based on a combination of flapper-nozzle system is presented in Fig. 3.5(b).

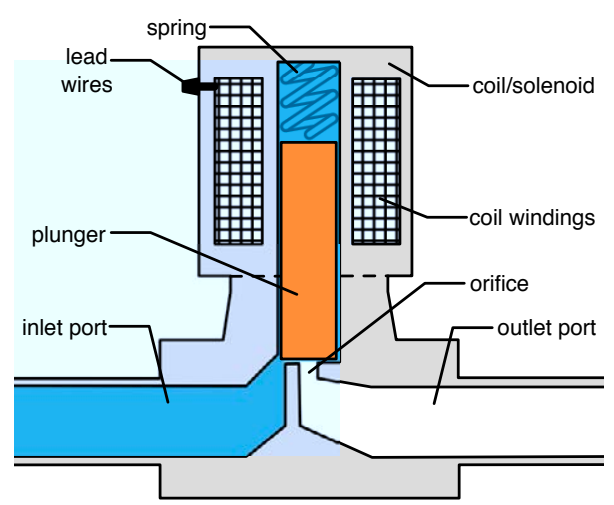

(a)

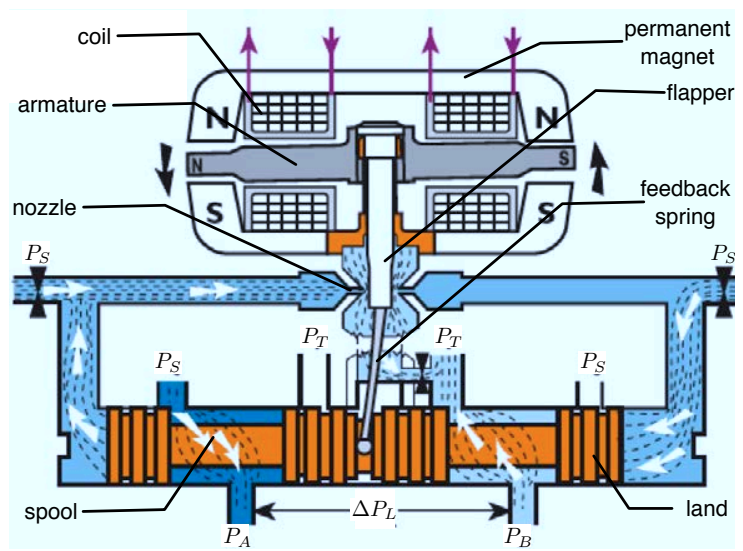

(b)

Figure 3.5: Fluid control valves. 3.5(a): Solenoid valve. (off configuration). 3.5(b): Two stage servo valve with four ways and three lands (4/3). Schematic diagram of its internal connection. (Scheme from the MOOG's technical data). 
The basis of its operation can be summarized as follows. An input current through the torque motor coils creates a magnetic force which causes a deflection of the armature/flapper assembly, restricting the fluid flow through one nozzle and diverts the flow to that end of the spool. The movement of the spool opens the supply pressure port $\left(P_{S}\right)$ to one control port while simultaneously opening the tank port $\left(P_{T}\right)$ to the other control port (e.g $P_{S} \rightarrow P_{A}$ and $P_{T} \rightarrow P_{B}$ ). The spool also pushes the end of the feedback spring creating an opposing torque on the armature/flapper assembly. The spool stops at a position where the torque generated by the feedback spring equals the torque due to the input current. Therefore, the spool position is proportional to the input current and, with constant pressure drop across the valve, the flow to the load is proportional to spool position.

A schematic diagram of its internal loops and dynamics is presented in Fig. ??. Depending on the level of detail, the complete model could require a 6 th order system (72).

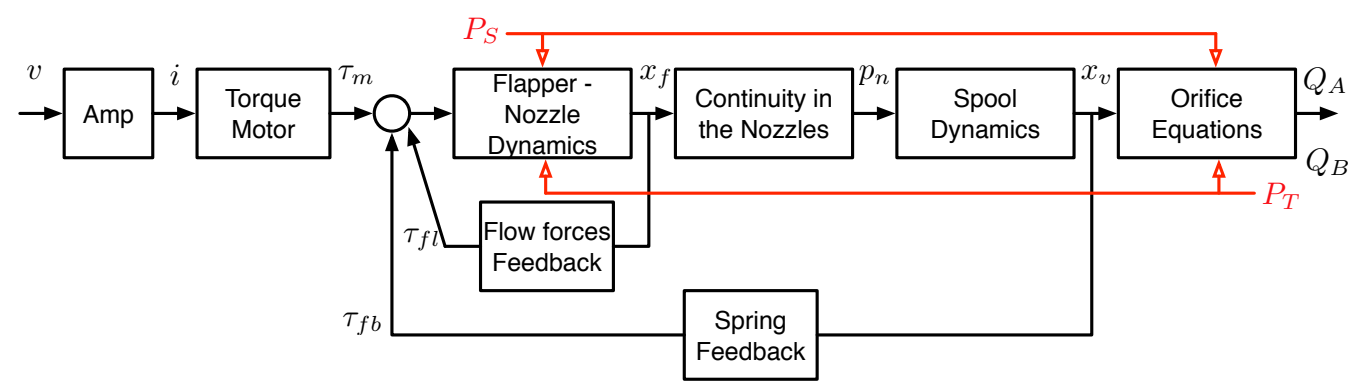

Figure 3.6: Schematic diagram of the dynamics of the servo-valve. $v$ voltage to the amplifier. $i$ current applied to the coil of the motor. $\tau_{m}$ torque generated by the motor. $\tau_{f l}$ torque due to flow forces at the flapper. $\tau_{f b}$ torque of the spring feedback. $x_{f}$ deflection of the flapper. $p_{n}$ pressure at the orifices of the nozzles. $x_{v}$ position of the spool.

\subsubsection{Actuators}

Hydraulics actuators are devices that drives loads by converting hydraulic energy provided by the pump into useful mechanical work. The mechanical power delivered to the load is managed by controlling the fluid pressure and flow rate by using various control valves.

Hydraulic actuators can be classified into three main groups: 


\section{HYDRAULIC SYSTEMS}

Hydraulic rotary actuators perform limited angular displacement. They can be based on rack and pinion drive, parallel piston, or vane approaches, achieving up to $360^{\circ}$ of rotation.

Hydraulic motors perform continuous rotary motion. They can be conceptually thought as reverse pumps, i.e. displacement machines converting the supplied hydraulic power into mechanical. Most of the pumps can be used as motor, however, the most commonly used are based on the vane and swash plate axial piston approaches.

Hydraulic cylinders perform linear motion. They mainly consist of the piston, a piston rod, and a cylinder, where the piston rod is extruded through the cylinder head by means of differential pressure at the faces of the piston. In particular, they can be classified into the following types:

- Single-acting cylinders: the piston is driven hydraulically only in one direction, which implies the need of an external restitution force for the other direction, usually a built-in spring.

- Double acting cylinders: the piston is driven hydraulically in both directions. The cylinder may be single rod, twin-rod symmetrical, or twin-rod nonsymmetrical.

- Tandem cylinders: duplicates the pressure force, for the same barrel diameter.

- Telescopic cylinders: provides long cylinder strokes with relatively small installation space. It can be single acting or double acting.

The basis of working of a generic cylinder can be summarized as follows. The incoming flow $Q_{A}$ through the Port A produces an increase on the pressure at chamber A that acts over the surface A of the piston. If this pressure is bigger than the pressure exerted by the oil fluid at the rod side of the piston and the external load, the oil will flow out of chamber B, producing the piston to move.

In an asymmetric double-acting cylinder, the effective surface at both sides of the piston differs, and it is usual to express them in term of their ratio, i.e. $A_{p}=A_{1} / A_{2}$. 


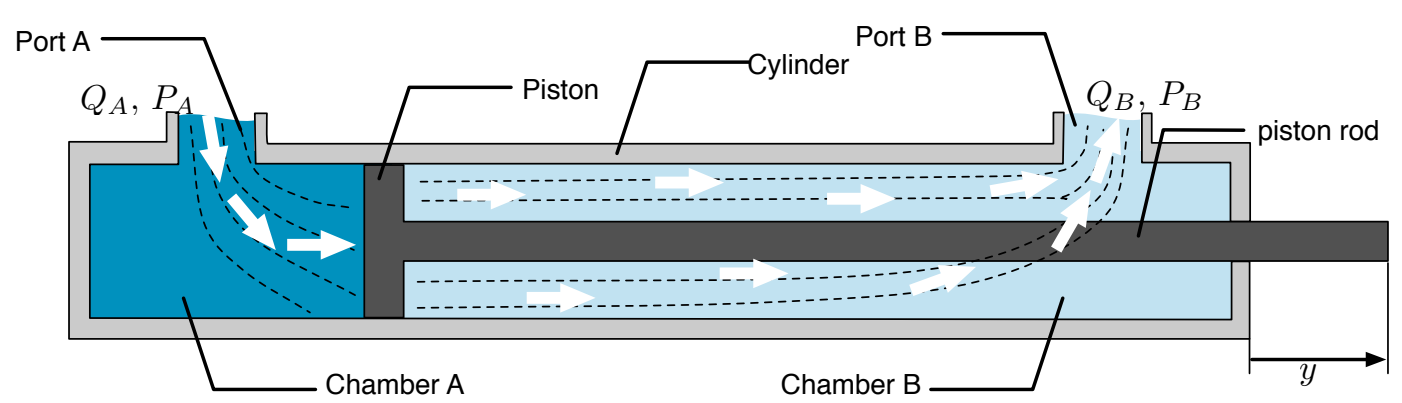

Figure 3.7: Schematic diagram of the internal chamber of the cylinder.

In particular, in a steady-state operation the dynamic of a cylinder is given by the following expressions:

$$
\begin{aligned}
F & =P_{A} A_{1}-P_{B} A_{2}-F_{f}, \\
\dot{y} & =\frac{Q_{A}-Q_{L}}{A_{p}}=\frac{Q_{B}-Q_{L}}{A_{p}},
\end{aligned}
$$

where $F_{f}$ is the friction force, and $Q_{L}$ is the internal leakage.

In transient response the effects of compressibility of the oil inside the chambers governed by the bulk modulus of the fluid, makes that the two oil columns act as two springs which causes the second order behavior which is always found in series with the integrating character of an hydraulic actuator. (72).

\subsection{Implementation of Hydraulic Actuation Systems}

Given the characteristics of the hydraulic actuation system, companies like Caterpillar@, John Deere(C), Komatsu(c) among other, rely on this technology for construction, forestry, material handler heavy machinery (see Fig. 3.8(a)).

Hydraulic system is also the election of many researchers and designers for their innovation, such as the bio-inspired four leg Big dog from Boston Dynamics (c) (see Fig. 3.9(a)), designed for carrying heavy load in rough-terrain.

Another example is the Roboclimber (107) ( see Fig. 3.9(b)), which is a quadruped walking and climbing developed for teleoperated service that could safely perform slope monitoring and consolidation tasks.

An interesting concept of a walking house from Wysing Arts Centre (see Fig.3.9(c)), based on a six legged hydraulic arrangement allows to persons to live a nomadic life, 


\section{HYDRAULIC SYSTEMS}

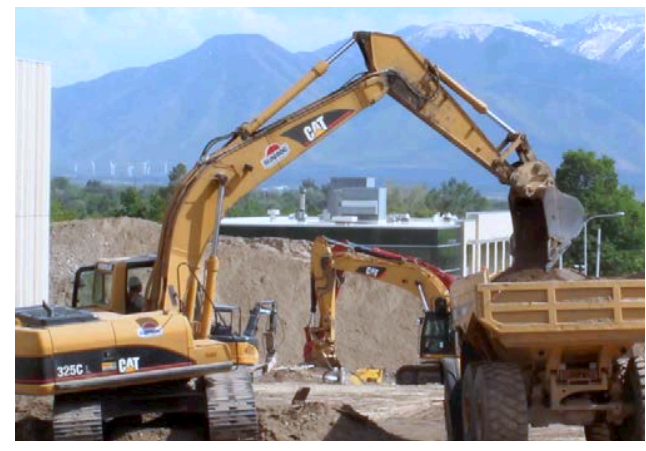

(a)

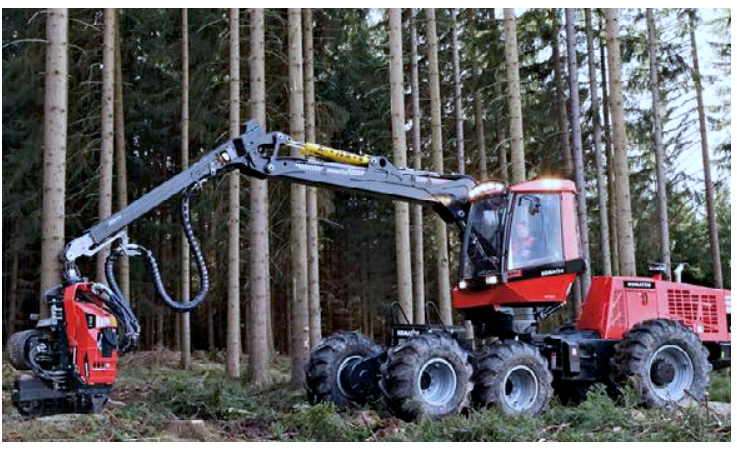

(b)

Figure 3.8: Used of hydraulic systems. 3.8(a): Caterpillar (c), 325C Excavator, $23000 \mathrm{Kg}$ maximum load. 3.8(b): Komatsu 941.1 forest final logging, 10m reach, maximum elevation torque $273 \mathrm{KNm}$.

moving slowly through all sorts of terrain with no dependency on existing infrastructure like roads.

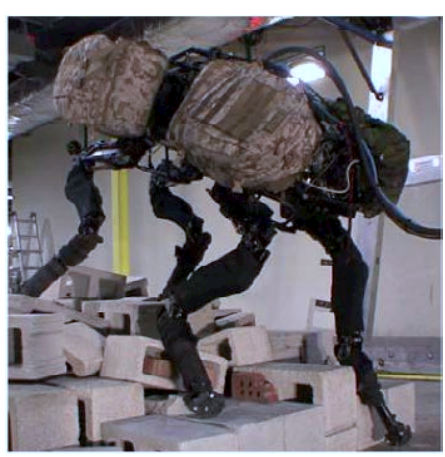

(a)

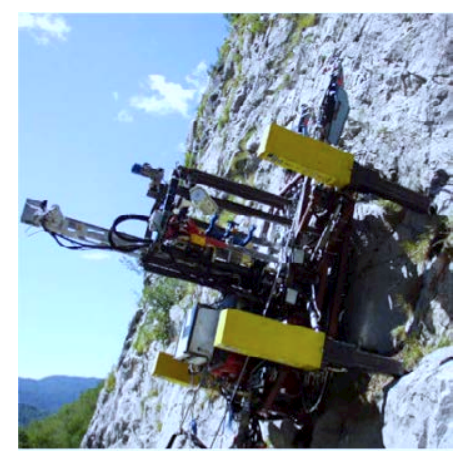

(b)

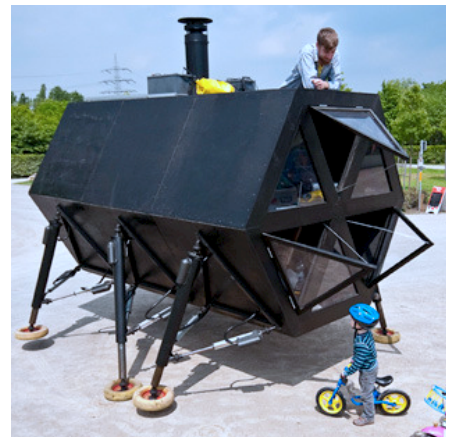

(c)

Figure 3.9: Implementation of hydraulic actuation on innovation. 3.9(a): big dog from Boston Dynamics(c). 3.9(b): Roboclimber. 3.9(c): N55 walking house concept from Wysing Arts Centre. 


\section{Part II}

\section{Hydraulic Actuator}





\title{
4
}

\section{Servo Hydraulic Actuator}

\author{
"... words are, in my not so humble opinion, \\ our most inexhaustible source of magic, capable \\ of both inflicting injury and remedying it ..." \\ Prof. Dumbledore.
}

\subsection{Introduction}

The core of every robotic manipulator are their actuators, therefore the performance of a robot will be always limited by the performance of the actuation systems.

Currently, there are many technologies available that permits the generation of force/moment, among them and perhaps the most used in robotics are the electrical, hydraulic and pneumatic technology. However, there is no comparison with the hydraulic system in terms of power generation - system's volume relation.

The main drawback of hydraulic actuation is that its components have complex nonlinear dynamics associated to the oil compressibility, oil flow through orifices, internal forces/pressure balances, internal leakage, friction effects and oil temperature dependency among others.

Building a mathematical model that includes all the system dynamics is not a trivial matter, several improvements have been made in this topic. 


\section{SERVO HYDRAULIC ACTUATOR}

In the current literature it can be found several approaches for describing this complex dynamic.

Complete model for the SHA is of great importance for the designer and optimization of the component (59) (58), however, for simulation and control simplified models are best suited.

As it is proved in (65) a linearized third order model is sufficient for feedback control design.

However, all models are simplified versions that consider only the governing dynamics. It is a general practice to neglect the internal dynamics of the servo-valve and assume that it behaves as a first or second order system (104). The nonlinear model can be derived from the physical laws, which can be linearized and depending of the assumptions considered it can be obtained a third or fourth order system (72) (149). If the cylinder is symmetric (i.e. both area are equal, also known as doubled rod cylinder), it is possible to combine the pressure at both chamber into a single equation defining an equivalent load flow and load pressure and thus simplifying the parameters of the third order model (101) (41). It is also very common to find fifth order model (77), where the dynamics of the spool of the servo-valve is also considered and approached to a second order system.

Another strategy used for modeling a hydraulic system is by means of the linear graph method. This approach allows a systematic generation of state-space equations, using three sets of equations.: element equations which describe the relationship between pressure and flow for the elementary hydraulic elements such as the inertial, capacitor and resistive element. compatibility equations result in pressure drop equations along a closed circuit and continuity equations result in flow continuity at systems nodes or closed surfaces.(40).

The Bondgraph method, is another powerful graphical tool for modelling complex systems even with interaction of several energy domains. It is based on the bidirectional exchange of energy between the elements (47).

In order to overcome the limitations of the simplified model and the time-variant parameters, in (35) it is introduced a fuzzy model, where the rules are taken from the dominant physical law of the system and they can learn from direct observation of its functioning. 
In this chapter, the hydraulic actuator of the PM is complete analyzed. First, a brief description of its elements and the basis of their operation are presented. Then, a complete model and a linearized model of the actuator is introduced. Experimental identification of a linear and a non linear model of the actuator is also presented. Finally, several control strategies are implemented in simulation and further validation in the real actuator.

\subsection{Servo Hydraulic Actuator Description}

The main parts that compose the hydraulic servo actuator are the servo-valve and the double effect cylinder. Position and force are measured by a linear digital encoder and a load cell at the distal end of the piston rod, respectively. An schematic diagram of the hydraulic circuit is presented in Fig. 4.1.

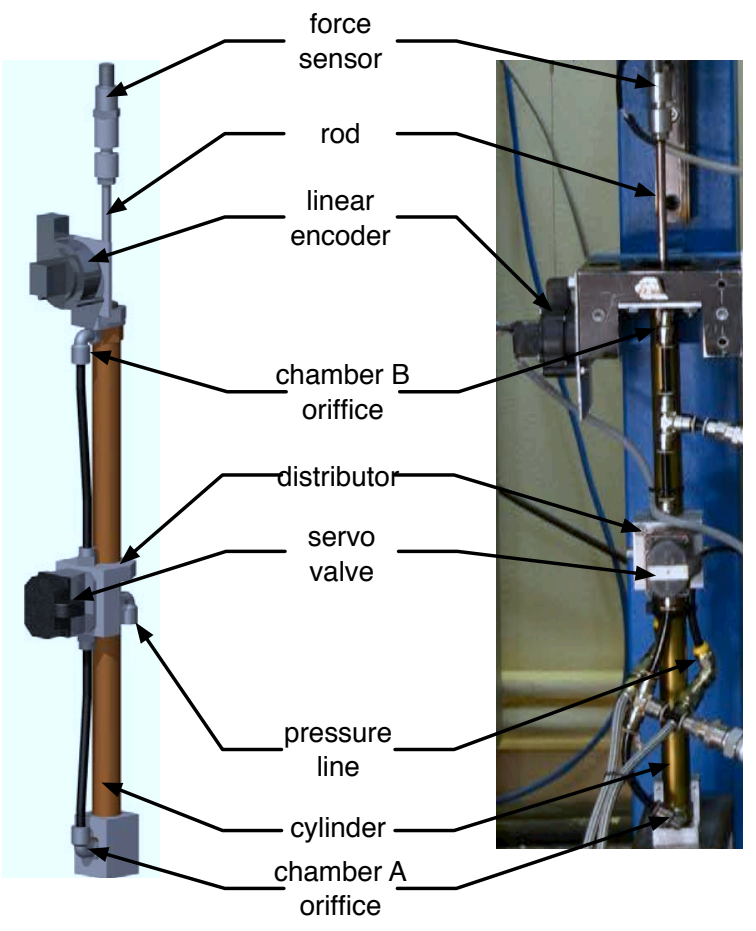

(a)

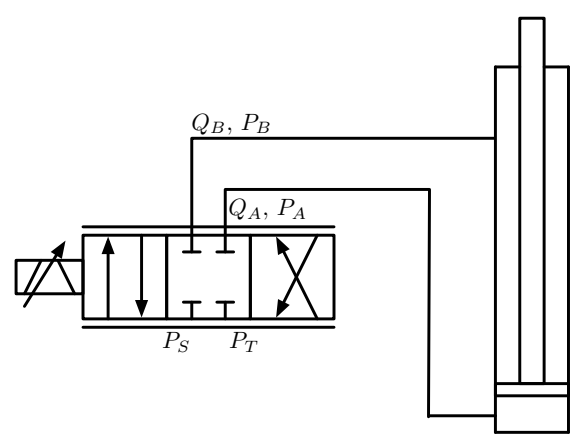

(b)

Figure 4.1: Schematic diagram of the servo hydraulic actuator circuit. 


\section{SERVO HYDRAULIC ACTUATOR}

\subsubsection{Servo-valve}

The servovalve is a Moog E050 series miniature servo-valve (see Fig. ??), which is composed by two stages, the pilot stage and the main stage. The pilot stage is a symmetrical double-nozzle and flapper configuration, driven by a double air gap torque that controls the spool of the output stage. The output stage is a closed-centre, four way, spool sliding directly in a corrosion resistant steel body. (See Fig. 3.5(b)).

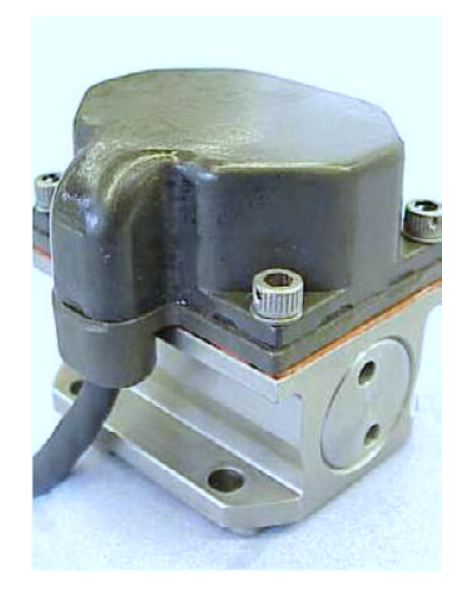

Figure 4.2: Proportional Servo-valve.

\subsubsection{Asymmetric double effect cylinder}

The cylinder is a CylVal 300-series double effect cylinder with a maximum stroke of $304 \mathrm{~mm}$, with $6 \mathrm{~mm}$ of inner radio and $3 \mathrm{~mm}$ of piston rod radio (see Fig. ??).

Since the oil in the two chamber is compressible, the two oil columns act as two springs, which causes the second order behavior which is always found in series with the integrating character of an hydraulic actuator. (72).

\subsection{Simplified Non linear Model}

Despite the fact that the complete model of the SHA represents an extensive insight of the physical behavior of the system, it depends of many parameters that can not be always accurately obtained. Therefore, the rigorousness of the model is lost since some of these parameters must be estimated. 


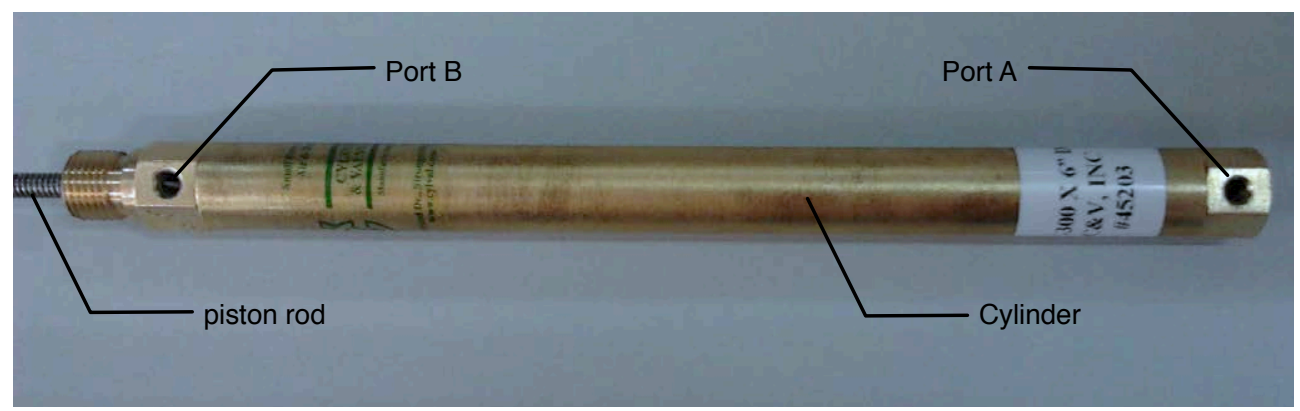

Figure 4.3: Photo of hydraulic cylinder.

On the other hand, it is usually possible to use a simplified dynamic model of the same order as the relevant dynamics of the real system with the dominant non-linearities included. Even though there will always remains a gap between the real system and the simplified model, for control design purposes, once that the model is validated it is useful.

In this work, the simplified model of the SHA is obtained adopting the following considerations:

- motor dynamics of the servo-valve can be neglected.

- flapper dynamics of the servo-valve can be neglected.

- spool dynamics of the servo-valve can be neglected.

- according to (72), the dynamics of the pipeline can be neglected if the length of the pipeline verifies:

$$
l<\frac{c}{10 f_{\max }}
$$

where $c$ is the sonic velocity (or wave speed) in oil, and $f_{\max }$ is the maximum working frequency. The dynamics of the pipelines are eliminated in the simplified model.

- the hydraulic station is ideal, therefore the pressure supply remains constant.

Based on these considerations, the dynamic of the SHA is simplified into the orifice equation for the servo-valve, the pressure dynamics at the cylinder, and the motion equation, as schematically depicted in Fig. 4.4. 


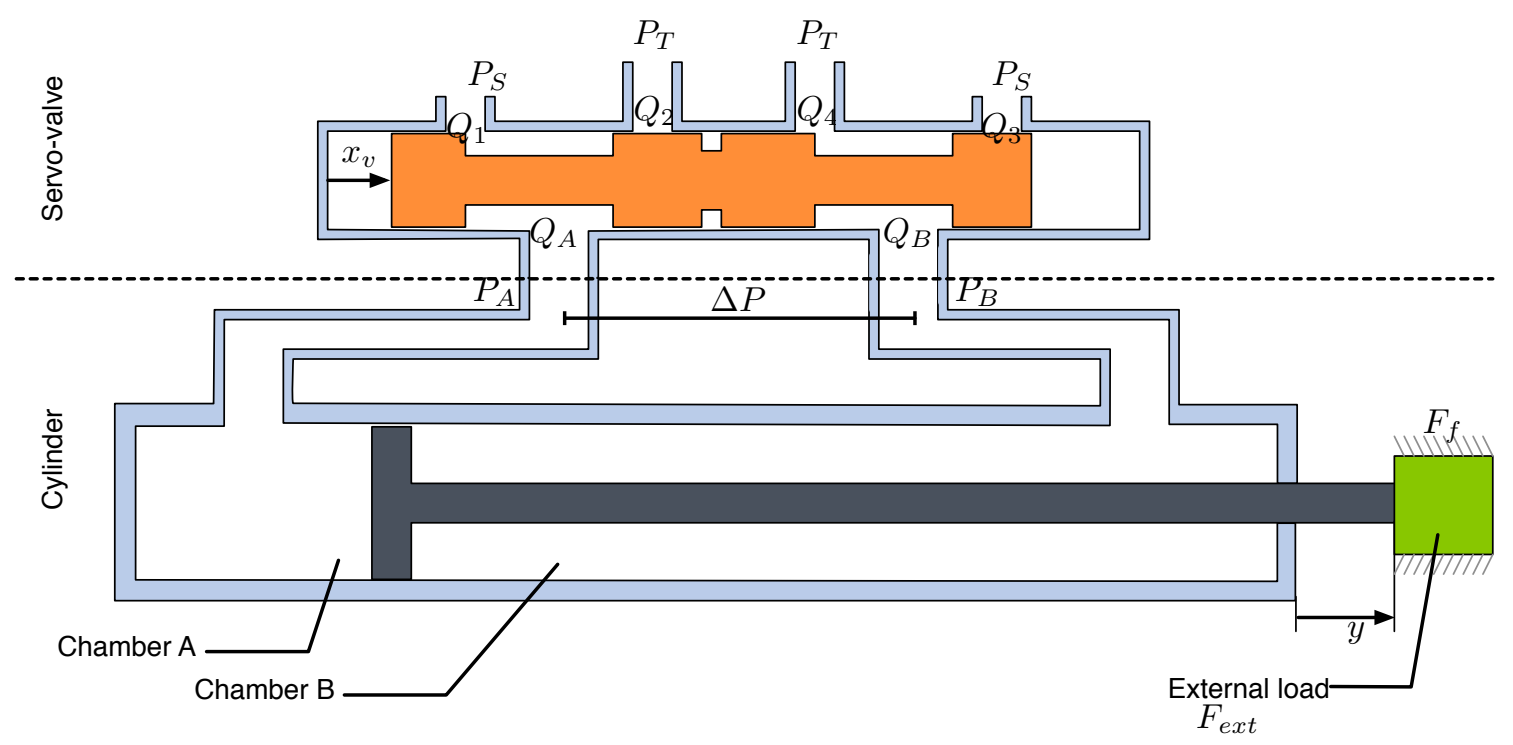

Figure 4.4: Schematic diagram of the simplified SHA.

\section{Orifice equation for the servo-valve}

Considering that a valve regulates the flows delivered through the control ports (namely $P_{A}$ and $P_{B}$ ) by modifying the aperture of its orifices, (3.1) can be modified considering linear ports (i.e. linear relationship between the valve spool position $x_{v}$ and the orifice area) and the direction of the pressure drop $\Delta P$ as follows:

$$
Q=Q\left(x_{v}, \Delta P\right)=c_{v} x_{v} \sqrt{|\Delta P|} \operatorname{sign}(\Delta P),
$$

where the flow coefficient $c_{v}$ for a servo-valve is given by

$$
c_{v}=\pi d_{v} \alpha_{d} \sqrt{\frac{2}{\rho}}
$$

and $d_{v}$ is the diameter of the valve spool.

Customizing (4.2) for a four-way spool valve with an ideal critical centre, the flow equations can be written as (see Fig. 4.4):

$$
\begin{aligned}
Q_{A} & =Q_{1}-Q_{2} \\
& =c_{v 1} \operatorname{sg}\left(x_{v}\right) \operatorname{sign}\left(P_{S}-P_{A}\right) \sqrt{\left|P_{S}-P_{A}\right|}-c_{v 2} \operatorname{sg}\left(-x_{v}\right) \operatorname{sign}\left(P_{A}-P_{T}\right) \sqrt{\left|P_{A}-P_{T}\right|}, \\
Q_{B} & =Q_{3}-Q_{4} \\
& =c_{v 3} \operatorname{sg}\left(-x_{v}\right) \operatorname{sign}\left(P_{S}-P_{B}\right) \sqrt{\left|P_{S}-P_{B}\right|}-c_{v 4} \operatorname{sg}\left(x_{v}\right) \operatorname{sign}\left(P_{B}-P_{T}\right) \sqrt{\left|P_{B}-P_{T}\right|},
\end{aligned}
$$


where the flow direction is determined by the sing of $x_{v}$, and $c_{v i}, i=1, \ldots, 4$ are the discharge coefficients, and the function $s g(x)$ is defined by:

$$
s g(x)=\left\{\begin{array}{lll}
x & \text { for } & x \geqslant 0 \\
0 & \text { for } & x<0
\end{array}\right.
$$

\section{Pressure dynamics}

The flow continuity at both chamber of the asymmetric cylinder yields (see Fig. 4.4):

$$
\begin{aligned}
Q_{A}-Q_{L i} & =\dot{V}_{A}+\frac{V_{A}}{\beta_{A}} \dot{P}_{A}, \\
Q_{B}-Q_{L i}-Q_{L e} & =\dot{V}_{B}+\frac{V_{B}}{\beta_{B}} \dot{P_{B}},
\end{aligned}
$$

where $Q_{L i}$ and $Q_{L e}$ denote the internal and external leakage flow respectively, $\beta_{A}$ and $\beta_{B}$ are the bulk modulus of the fluid at each chamber, and the volumes $V_{A}$ and $V_{B}$ of the chamber $A$ and $B$ can be expressed as:

$$
\begin{aligned}
& V_{A}=V_{A 0}+x_{p} A_{p}, \\
& V_{B}=V_{B 0}-\alpha x_{p} A_{p} .
\end{aligned}
$$

Since the pipeline's dynamics is neglected, the volume of the pipelines that connect the servovalve and the cylinder are included in the initial volume $V_{A 0}$ and $V_{B 0}$.

Taking the time derivatives of (4.9) and (4.10), as follows:

$$
\begin{aligned}
& \dot{V}_{A}=A_{p} \dot{x}_{p}, \\
& \dot{V}_{B}=-\alpha A_{p} \dot{x}_{p},
\end{aligned}
$$

and considering that the internal and external leakage can be neglected (i.e. $Q_{L i}=$ $\left.Q_{L e}=0\right)$, then (4.7) and (4.8) can be rearranged as follows,

$$
\begin{aligned}
& \dot{P}_{A}=\frac{\beta_{A}}{V_{A}}\left(Q_{A}-A_{p} \dot{x}_{p}\right), \\
& \dot{P}_{B}=\frac{\beta_{B}}{V_{B}}\left(Q_{B}+\alpha A_{p} \dot{x}_{p}\right),
\end{aligned}
$$

\section{Equation of motion}

The motion of the external load arises by the action of the hydraulic cylinder, and it can be found by applying Newton's second law on the piston as follows,

$$
A_{p}\left(P_{A}-\alpha P_{B}\right)-F_{f}\left(\dot{x}_{p}\right)-F_{e}=m_{t} \ddot{x}_{p}
$$




\section{SERVO HYDRAULIC ACTUATOR}

where $F_{f}$ is the friction force generated as consequence of the relative movement between the interacting elements, and $F_{e}$ are all the external forces which are considered now on as perturbation (e.g. force due to gravity, interactions with the environment, etc.). The total mass $m_{t}$ considers the mass of the piston $m_{p}$ and the mass of the fluid in each chamber and in the pipelines $m_{A, f l}$ and $m_{B, f l}$

$$
m_{t}=m_{p}+m_{A, f l}+m_{B, f l}
$$

the mass of the fluid can be calculated according to

$$
\begin{aligned}
& m_{A, f l}=\rho\left[V_{p l, A}+\left(x_{p 0}+x_{p}\right) A_{p}\right] \\
& m_{B, f l}=\rho\left[V_{p l, B}+\left(x_{p 0}+x_{p}\right) A_{p}\right]
\end{aligned}
$$

Therefore, defining the following estates and input variables,

$$
\begin{aligned}
& x_{1}=y \\
& x_{2}=\dot{y} \\
& x_{3}=P_{A} \\
& x_{4}=P_{B}
\end{aligned}
$$

the state model is given by

$$
\left\{\begin{array}{l}
\dot{x}_{1}=x_{2} \\
\dot{x}_{2}=\frac{1}{m_{t} x_{1}}\left[A_{p}\left(x_{3}-\alpha x_{4}\right)-F_{f}\left(x_{2}\right)-u_{2}\right], \\
\dot{x}_{3}=\frac{\beta_{A}}{V_{A}}\left[Q_{A}\left(x_{3}, u_{1}\right)-A_{p} x_{2}\right] \\
\dot{x}_{4}=\frac{\beta_{B}}{V_{B}}\left[Q_{B}\left(x_{4}, u_{1}\right)+\alpha A_{p} x_{2},\right]
\end{array}\right.
$$

where $Q_{A}$ and $Q_{B}$ is given by (4.7) and (4.8).

\subsection{Linearized Model}

The model presented in the previous sections requires the measurement of $P_{A}$ and $P_{B}$. This signals are not available in the robotic application, instead each actuator is provided by a force sensor. Therefore, it is a must to develop a model based on this variable.

Lets remember that the force exerted by the piston is related to the pressure at the chambers as follows:

$$
F_{L}=A_{p} P_{L}
$$


where $P_{L}=P_{A}-\alpha P_{B}$ is defined as the load pressure.

Therefore, based on this definition of the load pressure, the expressions that govern the dynamics of the system must be rearranged in order to replace the variables $P_{A}$ and $P_{B}$ with the term $P_{L}$.

Lets remember that in steady state velocity, the flow through the orifices of the piston's chamber is given by,

$$
\begin{aligned}
& Q_{A}=A_{p} \dot{y}, \\
& Q_{B}=\alpha A_{p} \dot{y} .
\end{aligned}
$$

Equating (4.25) and (4.26) with the orifice equation of the servo-valve given by (4.7) and (4.8), and defining the expressions for positive and negative movement as follows:

$$
\begin{aligned}
& \left\{\begin{array}{l}
A_{p} \dot{y}=x_{v} c_{v 1} \sqrt{P_{S}-P_{A}} \\
\alpha A_{p} \dot{y}=-x_{v} c_{v 4} \sqrt{P_{B}-P_{T}} \quad \text { for } x_{v} \geqslant 0,
\end{array}\right. \\
& \left\{\begin{array}{l}
A_{p} \dot{y}=x_{v} c_{v 2} \sqrt{P_{A}-P_{T}} \\
\alpha A_{p} \dot{y}=-x_{v} c_{v 3} \sqrt{P_{S}-P_{B}} \quad \text { for } x_{v}<0 .
\end{array}\right.
\end{aligned}
$$

the pressure at the cylinder's chambers can be expressed in terms of the piston's position and the input signal of the servo-valve:

$$
\begin{gathered}
P_{A}=\left\{\begin{array}{cc}
P_{S}-R_{h 1} A_{p}^{2} \dot{y}^{2} & \text { for } x_{v} \geqslant 0 \\
P_{T}+R_{h 2} A_{p}^{2} \dot{y}^{2} & \text { for } x_{v}<0
\end{array}\right. \\
P_{B}= \begin{cases}P_{T}+R_{h 4} \alpha^{2} A_{p}^{2} \dot{y}^{2} & \text { for } x_{v} \geqslant 0 \\
P_{S}-R_{h 3} \alpha^{2} A_{p}^{2} \dot{y}^{2} & \text { for } x_{v}<0\end{cases}
\end{gathered}
$$

where $R_{h 1}=\left(x_{v}^{2} c_{v 1}^{2}\right)^{-1}, R_{h 2}=\left(x_{v}^{2} c_{v 2}^{2}\right)^{-1}, R_{h 3}=\left(x_{v}^{2} c_{v 3}^{2}\right)^{-1}, R_{h 4}=\left(x_{v}^{2} c_{v 4}^{2}\right)^{-1}$.

Considering the definition of the load pressure (i.e. $P_{L}=P_{A}-\alpha P_{B}$ ), it can found that:

$$
\dot{y}^{2}= \begin{cases}\frac{1}{A_{p}^{2}\left(R_{h 1}+\alpha^{3} R_{h 4}\right)}\left(P_{S}-\alpha P_{T}-P_{L}\right) & \text { for } x_{v} \geqslant 0 \\ \frac{1}{A_{p}^{2}\left(R_{h 2}+\alpha^{3} R_{h 3}\right)}\left(\alpha P_{S}-P_{T}+P_{L}\right) & \text { for } x_{v}<0\end{cases}
$$


Taking (4.31) into (4.29) and (4.30), the pressure at the piston's chambers can be expressed in terms of the load pressure $P_{L}$ as follows:

$$
\begin{gathered}
P_{A}= \begin{cases}\frac{1}{R_{h 1}+\alpha^{3} R_{h 4}}\left(\alpha^{3} R_{h 4} P_{s}+\alpha R_{h 1} P_{T}+R_{h 1} P_{L}\right) & \text { for } x_{v} \geqslant 0 \\
\frac{1}{R_{h 2}+\alpha^{3} R_{h 3}}\left(\alpha R_{h 2} P_{s}+\alpha^{3} R_{h 3} P_{T}+R_{h 2} P_{L}\right) & \text { for } x_{v}<0\end{cases} \\
P_{B}= \begin{cases}\frac{1}{R_{h 1}+\alpha^{3} R_{h 4}}\left(\alpha^{2} R_{h 4} P_{s}+R_{h 1} P_{T}-\alpha^{2} R_{h 4} P_{L}\right) & \text { for } x_{v} \geqslant 0 \\
\frac{1}{R_{h 2}+\alpha^{3} R_{h 3}}\left(R_{h 2} P_{s}+\alpha^{2} R_{h 3} P_{T}-\alpha^{2} R_{h 3} P_{L}\right) & \text { for } x_{v}<0 .\end{cases}
\end{gathered}
$$

Therefore, implementing the linearization technique on the dominant dynamic equations of the system, it can be formulated an even simpler model defined in an operating point.

Taking an operating point $P_{0}=\left[x_{v 0}, P_{A 0}, P_{B 0}\right]$, and assuming first dominance for differentials considering a Taylor series expansion, the set of linearized equations are stated as follows,

\section{Linearized pressure equation .}

$$
\begin{gathered}
\delta P_{A}= \begin{cases}\frac{R_{h 1}}{R_{h 1}+\alpha^{3} R_{h 4}} \delta P_{L} & \text { for } x_{v} \geqslant 0 \\
\frac{R_{h 2}}{R_{h 2}+\alpha^{3} R_{h 3}} \delta P_{L} & \text { for } x_{v}<0\end{cases} \\
\delta P_{B}= \begin{cases}-\frac{\alpha^{2} R_{h 4}}{R_{h 1}+\alpha^{3} R_{h 4}} \delta P_{L} & \text { for } x_{v} \geqslant 0 \\
-\frac{\alpha^{2} R_{h 3}}{R_{h 2}+\alpha^{3} R_{h 3}} \delta P_{L} & \text { for } x_{v}<0\end{cases}
\end{gathered}
$$

\section{Linearized orifice equation for the servo-valve .}

$$
\begin{aligned}
& \delta Q_{A}=K_{x A} \delta x_{v}+K_{P A} \delta P_{A}, \\
& \delta Q_{B}=K_{x B} \delta x_{v}+K_{P_{B}} \delta P_{B},
\end{aligned}
$$

where $K_{x A}, K_{x_{B}}$, are the flow-gains given by:

$$
K_{x A}=\left.\frac{\partial Q_{A}}{\partial x_{v}}\right|_{P_{0}}= \begin{cases}c_{v 1} \sqrt{P_{S}-P_{A 0}} & \text { for } x_{v}>0, \\ -c_{v 2} \sqrt{P_{A 0}-P_{T}} & \text { for } x_{v}<0,\end{cases}
$$




$$
K_{x B}=\left.\frac{\partial Q_{B}}{\partial x_{v}}\right|_{P_{0}}= \begin{cases}-c_{v 4} \sqrt{P_{B 0}-P_{T}} & \text { for } x_{v}>0, \\ c_{v 3} \sqrt{P_{S}-P_{B 0}} & \text { for } x_{v}<0,\end{cases}
$$

$K_{P A}$ and $K_{P B}$ are the flow-pressure coefficients,

$$
\begin{gathered}
K_{P A}=\left.\frac{\partial Q_{A}}{\partial P_{A}}\right|_{P_{0}}= \begin{cases}\frac{-c_{v 1} x_{v 0}}{2 \sqrt{P_{S}-P_{A 0}}} & \text { for } x_{v}>0, \\
\frac{-c_{v 2} x_{v 0}}{2 \sqrt{P_{A 0}-P_{T}}} & \text { for } x_{v}<0,\end{cases} \\
K_{P B}=\left.\frac{\partial Q_{B}}{\partial P_{B}}\right|_{P_{0}}= \begin{cases}\frac{-c_{v 4} x_{v 0}}{2 \sqrt{P_{B 0}-P_{T}}} & \text { for } x_{v}>0, \\
\frac{-c_{v 3} x_{v 0}}{2 \sqrt{P_{S}-P_{B 0}}} & \text { for } x_{v}<0 .\end{cases}
\end{gathered}
$$

If the geometry of all the orifices of the servo-valve are identical, then $c_{v 1}=c_{v 2}=$ $c_{v 3}=c_{v 4}=c_{v}$.

\section{Linearized pressure dynamics .}

Adopting that fluid flows into chamber A and flows out of chamber B while the pistons's rods extends with positive velocity, then:

$$
\begin{aligned}
& \delta Q_{A}=A_{p} \delta \dot{y}+\frac{V_{A 0}}{\beta} \frac{d}{d t}\left[\delta P_{A}\right], \\
& \delta Q_{B}=\alpha A_{p} \delta \dot{y}-\frac{V_{B 0}}{\beta} \frac{d}{d t}\left[\delta P_{B}\right] .
\end{aligned}
$$

\section{Linearized equation of motion .}

In order to simplify the model, it is considered that the friction is governed by the classic static + viscous friction model given by $F_{f}(y)=F_{s}+\sigma \dot{y}$. Therefore, the linearized equation of motion is given by:

$$
A_{p}\left(\delta P_{A}-\alpha \delta P_{B}\right)-\delta F_{e x t}-\delta F_{f}=m \delta \ddot{y}
$$

Combining (4.36), (4.37), (4.42), (4.43), it can be found:

$$
\begin{aligned}
& K_{x_{A}} \delta x_{v}+K_{P_{A}} \delta P_{A}=A_{p} \delta \dot{y}+\frac{V_{A 0}}{\beta} \frac{d}{d t}\left[\delta P_{A}\right], \\
& K_{x_{B}} \delta x_{v}+K_{P_{B}} \delta P_{B}=\alpha A_{p} \delta \dot{y}-\frac{V_{B 0}}{\beta} \frac{d}{d t}\left[\delta P_{B}\right] .
\end{aligned}
$$

Solving them for $\frac{d}{d t}\left[\delta P_{A}\right]=\delta \dot{P}_{A}$ and $\frac{d}{d t}\left[\delta P_{B}\right]=\delta \dot{P}_{B}$,

$$
\begin{aligned}
\delta \dot{P}_{A} & =\frac{\beta}{V_{A 0}}\left(K_{x_{A}} \delta x_{v}+K_{P_{A}} \delta P_{A}-A_{p} \delta \dot{y}\right), \\
-\delta \dot{P}_{B} & =\frac{\beta}{V_{B 0}}\left(K_{x_{B}} \delta x_{v}+K_{P_{B}} \delta P_{B}-\alpha A_{p} \delta \dot{y}\right) .
\end{aligned}
$$


Recalling that the load pressure was defined as: $P_{L}=P_{A}-\alpha P_{B}$, its time derivative will be given as,

$$
\dot{P}_{L}=\dot{P}_{A}-\alpha \dot{P}_{B}
$$

Replacing (4.47) and (4.48) in (4.49), the load pressure dynamics can be stated in terms of the servo-valve sensibilities constants as follows,

$$
\delta \dot{P}_{L}=K_{x} \delta x_{v}-K_{d} \delta \dot{y}+K_{p} \delta P_{L}
$$

where:

$$
\begin{aligned}
K_{x} & =\frac{\beta_{A}}{V_{A}} K_{x A}+\alpha \frac{\beta_{B}}{V_{B}} K_{x B} \\
K_{d} & =A_{p}\left(\frac{\beta_{A}}{V_{A}}+\alpha^{2} \frac{\beta_{B}}{V_{B}}\right) \\
K_{p} & =\frac{\beta_{A}}{V_{A}} K_{P A} R_{i}-\alpha \frac{\beta_{B}}{V_{B}} K_{P B} R_{j}
\end{aligned}
$$

and

$$
\begin{gathered}
R_{i}= \begin{cases}\frac{R_{h 1}}{R_{h 1}+\alpha^{3} R_{h 4}} & \text { for } x_{v} \geqslant 0 \\
\frac{R_{h 2}}{R_{h 2}+\alpha^{3} R_{h 3}} & \text { for } x_{v}<0\end{cases} \\
R_{j}= \begin{cases}-\frac{\alpha^{2} R_{h 4}}{R_{h 1}+\alpha^{3} R_{h 4}} & \text { for } x_{v} \geqslant 0 \\
-\frac{\alpha^{2} R_{h 3}}{R_{h 2}+\alpha^{3} R_{h 3}} & \text { for } x_{v}<0\end{cases}
\end{gathered}
$$

Therefore, based on (4.44) and (4.50), and defining the following states and inputs variables:

$$
\begin{aligned}
& x_{1}=\delta y \\
& x_{2}=\delta \dot{y} \\
& x_{3}=\delta P_{L} \\
& u_{1}=\delta x_{v} \\
& u_{2}=\delta F_{e}
\end{aligned}
$$

the following state variable model describes the governing dynamics of the servo hydraulic actuator.

$$
\left[\begin{array}{c}
\dot{x}_{1} \\
\dot{x}_{2} \\
\dot{x}_{3}
\end{array}\right]=\left[\begin{array}{ccc}
1 & 0 & 0 \\
0 & -\frac{\sigma}{m} & \frac{A_{p}}{m} \\
0 & -K_{d} & K_{p}
\end{array}\right]\left[\begin{array}{l}
x_{1} \\
x_{2} \\
x_{3}
\end{array}\right]+\left[\begin{array}{c}
0 \\
0 \\
K_{x}
\end{array}\right] u_{1}+\left[\begin{array}{c}
0 \\
-\frac{1}{m} \\
0
\end{array}\right] u_{2} .
$$




\subsubsection{Model Analysis}

In order to simplify the analysis of the model, the Laplace transform (i.e. $\mathcal{L}(\cdot))$ is applied on (4.44) and (4.50), and the following expressions in terms of the complex variable $s$ are obtained:

$$
\begin{aligned}
s \Delta P_{L} & =K_{x} \Delta X-K_{d} \Delta V+K_{p} \Delta P_{L}, \\
A_{p} \Delta P_{L} & =m s \Delta V+\sigma \Delta V+\Delta F_{e},
\end{aligned}
$$

where $\Delta X(s)=\mathcal{L}\left\{x_{v}(t)\right\}, \Delta P_{L}(s)=\mathcal{L}\left\{\delta P_{L}(t)\right\}, \Delta F_{e}(s)=\mathcal{L}\left\{\delta F_{e}(t)\right\}, \Delta V(s)=$ $\mathcal{L}\{\delta \dot{y}(t)\}$. Solving (4.62) for $\Delta P L$, and replacing it in (4.63), the governing dynamics of the system can be expressed as follows (126):

$$
\Delta V=\frac{A_{p} K_{x}}{s^{2} b_{2}+s b_{1}+b_{0}} \Delta X-\frac{s-K_{p}}{s^{2} b_{2}+s b_{1}+b_{0}} \Delta F_{e}
$$

where:

$$
\begin{aligned}
& b_{2}=m \\
& b_{1}=-m K_{p}+\sigma \\
& b_{0}=A_{p} K_{d}-\sigma K_{p}
\end{aligned}
$$

Recalling that $\Delta V=\frac{1}{s} \Delta Y$, and expressing the denominator in the canonical form $s\left(s^{2}+2 \zeta w_{n} s+w_{n}^{2}\right),(4.64)$ can be rearranged as :

$$
\Delta Y=\frac{k_{01} b_{0}^{\prime}}{s\left(s^{2}+b_{1}^{\prime} s+b_{0}^{\prime}\right)} \Delta X-\frac{k_{02} b_{0}^{\prime}\left(s+z_{0}\right)}{s\left(s^{2}+b_{1}^{\prime} s+b_{0}^{\prime}\right)} \Delta F_{e},
$$

where:

$$
\begin{aligned}
b_{1}^{\prime} & =-K_{p}+\frac{\sigma}{m}, \\
b_{0}^{\prime} & =\frac{A_{p} K_{d}-\sigma K_{p}}{m}, \\
k_{01} & =\frac{A_{p} K_{x}}{m b_{0}^{\prime}}, \\
z_{0} & =-K_{p}, \\
k_{02} & =\frac{1}{m b_{0}^{\prime}} .
\end{aligned}
$$

From (4.68), it can be seen that the piston's position $(\Delta Y)$ responds not only on the input signal of the servo-valve $(\Delta X)$ but also of the external perturbations $\left(\Delta F_{e}\right)$. 


\section{SERVO HYDRAULIC ACTUATOR}

For a first analysis, let us assume that there is no external perturbation $\left(\Delta F_{e}=0\right)$, and let us define the transfer function $G(s)=\Delta Y(s) / \Delta X(s)$. This reduced system $G(s)$ posses a pole at the origin, revealing the integrating behavior of the system, i.e. a constant input $\Delta X$, after it transient response produces a displacement at constant velocity. The two other poles form a conjugate complex pole pair (72), and they define most of the system's dynamics. The natural frequency $w_{n}$ and damping $\zeta$ of these pair of poles are given by (4.74) and (4.75) respectively.

$$
\begin{aligned}
w_{n} & =\sqrt{b_{0}^{\prime}}, \\
& =\sqrt{\frac{K_{d} A_{p}-\sigma K_{p}}{m}}, \\
\zeta=\frac{1}{2} \frac{b_{1}^{\prime}}{\sqrt{b_{0}^{\prime}}} & \\
= & \frac{\sigma-m K_{p}}{2 \sqrt{m\left(K_{d} A_{p}-\sigma K_{p}\right)}} .
\end{aligned}
$$

From these expression, it can be seen that the natural frequency depends on the mass $(m)$ and the stiffness of the cylinder's chamber $\left(K_{d}\right.$ and $\left.K_{p}\right)$. The latter one is position dependent. Therefore, for a given position, the location of the complex pole pair tends to the origin of the complex plane $(\mathbb{C})$ as $m$ increases. On the other hand, the dumping is governed by the viscous friction $\sigma / m$.

With regard to the stability of $G(s)$ and according to Routh-Hurtwitz criteria, $b_{1}^{\prime}$ and $b_{0}^{\prime}$ should be positive and non zero. Thus, the following inequalities should be met:

$$
\begin{aligned}
b_{1}^{\prime} & >0, \\
-K_{p}+\frac{\sigma}{m} & >0, \\
K_{p} & <\frac{\sigma}{m} . \\
b_{0}^{\prime} & >0, \\
\frac{K_{d} A_{p}-\sigma K_{p}}{m} & >0, \\
K_{d} & >\frac{\sigma K_{p}}{A_{p}} .
\end{aligned}
$$


From (4.76) it can be seen that since $\sigma>0$ and $m>0$, then $K_{p}<0$, therefore from (4.77) and considering that $A_{p}>0$, then $K_{d}$ is positive.

The current zero $\left(z_{0}\right)$ in (4.68) depends of the flow pressure coefficients (see (4.72)), and it is located on the left half plane $\left(\mathbb{C}^{-}\right)$of the complex plane $(\mathbb{C})$. Even more, the zero is located at the left of the complex pole pair, since the real part of the complex pole pair $\left(\operatorname{Re}\left(p_{i}\right)\right)$ is given by:

$$
\begin{aligned}
\operatorname{Re}\left(p_{i}\right) & =\zeta w_{n}, \\
& =-\frac{1}{2}\left(K_{p}+\frac{\sigma}{m}\right),
\end{aligned}
$$

and therefore: $\operatorname{Re}\left(p_{i}\right)>z_{0}$.

\subsection{Conclusions}

A hydraulic actuator is a complex device that has several interacting elements providing closed-loop physical effects requiring higher order differential equations. Even though, these equations may gather all the dynamics of the system, their parameters are not always easily found and in any case they should be estimated. Therefore simplified model are mostly used. Furthermore, it is common practice to reduce the problem even more by linearizing it. The final linear model is a third order model with two important characteristics: the model has a pole at the origin and the model can be separated in two parts. One of them is the related directly with the command signal of the displacement of the rod. The other, remarks the relation of external perturbation on the displacement of the rod. 
4. SERVO HYDRAULIC ACTUATOR 


\section{5}

\section{Experimental Identification of SHA}

"... Clark Kent is how Superman sees us... he is weak, he is unsure, he is a coward. Clark Kent is the critic to the hole human race..."

\subsection{Introduction}

In the previous chapter the linear model of the servo-hydraulic actuator was introduced, and a brief analysis was presented. However, this basic and global knowledge of the system is not enough in order to design a proper controller for the actuator.

Even though some of the parameters that are involved in the model can be obtained from catalogues it is recommended (when possible), to identified them in the real system.

The identification process consist on exciting the system with a proper input signal, and finding the characterization of the input-output behavior of the system. There are several techniques that permits the characterization of the system. However, all of them rely on the definition of a model and parameters (i.e., state variable, pole-zero, etc.), the searching criteria (i.e. jacobian based search, neural networks, fuzzy logic, 


\section{EXPERIMENTAL IDENTIFICATION OF SHA}

etc.), and a cost function. The selection of the methodology depends on one's expertise and the prior knowledge of the system.

In (73) deals with the identification of nonlinear models in observer canonical form of hydraulic servo-drives, implementing modified Recursive Instrumental Variables algorithm in order to avoid unsuitable initial estimation that leads to convergence and singularity problems.

Frequency identification techniques are implemented in (87), (90).

ARX and ARMAX structures are used in (126) (88), with a multisine input.

In this chapter, the identification of the servo-hydraulic actuator is presented. First, the methodology implemented is introduced, then the experiments are presented and the processing is discussed. Finally the parameters as showed and discussed.

\subsection{Identification Process}

The identification process is not straight forward, it is an iterative process that requires common sense and intuition to get to the correct results. The procedure implemented in this work is schematized in Fig. 5.1.

As it can be observed, the process can be separated into three main steps:

1. Experimentation: this is perhaps the most critical step during the identification process, since it requires the definition of an experiment from whom the system's dynamics could be extracted. The experiment is detailed in 5.2.1.

2. First ID: in this step a first identification of some parameter are carried on. As it is depicted in Fig. 5.1, two different set of parameter are identified. The first set of parameters are related with the piston's motion dynamics (i.e. the total mass, the viscous friction and external perturbation: $m, \sigma, F_{e}$, respectively) detailed in 5.2.3 . The second set of parameters corresponds to the open-loop transfer function $G(s)$ that relates the input-output data detailed in 5.2.2. The parameters are separated into these two set and perform individually, in order to minimize the error estimation.

3. Optimization: in the optimization step, the sensibilities constants $\left(K_{p}, K_{d}\right.$ and $\left.K_{x}\right)$ are calculated from the identified motion's dynamics parameters and the open loop transfer function. Based on these constants and motion dynamics 


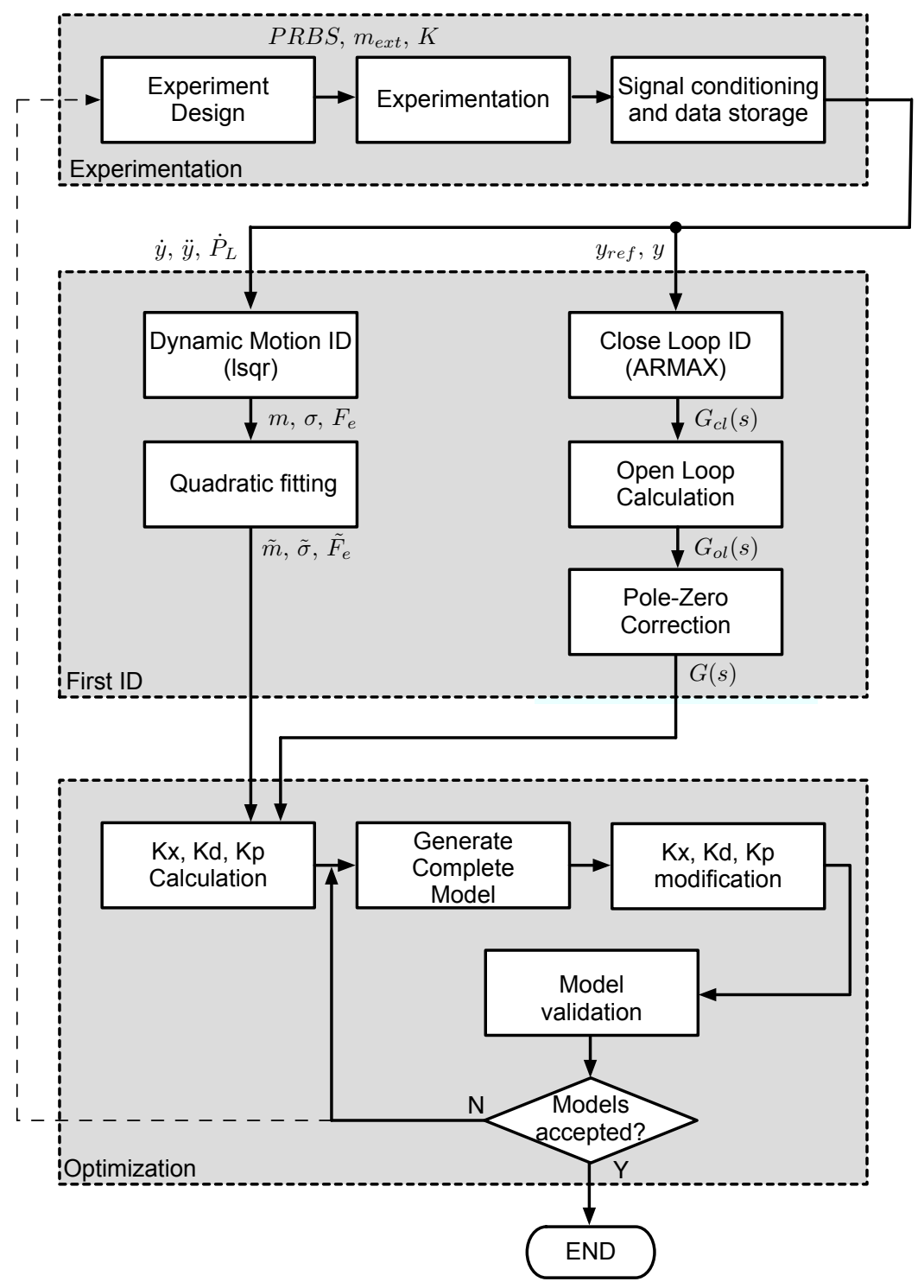

Figure 5.1: Identification Process.

parameter, the linearized model is generated and the validated. Variations on $K_{p}, K_{d}$ and $K_{x}$ are carried on in order to maximize the accuracy of the model $($ see 5.2 .4$)$. 


\section{EXPERIMENTAL IDENTIFICATION OF SHA}

\subsubsection{Experiment Design}

The model presented in the previous chapter was obtained implementing a linearization technique over the governing dynamics of the system, resulting in a model that depends both on the position and pressure at the chambers. Thus, the system has be identified for several position and pressure.

The system (as it was shown in the linearized model (4.68)) is a velocity system (i.e. it presents a pole at the origin), and as a consequence is not possible to identified the model at a given position of the piston in open loop manner.

Therefore the experimental identification of the linearized model must be performed in close loop with a position controller. For identification purposes, a position proportional controller is more than adequate. However, the selection of the proportional term must be chosen in order to obtain an underdamped response providing as much dynamics as possible from the system. Under this consideration all the amplitudes and frequencies and all their combinations must be represented in the input signal. Then, a pseudo-random multilevel signals (PRMS) is implemented as input signal.

On the other hand, in order to provide different pressure points, the experiments are performed for several external load.

During the experimentation, the supply pressure $\left(P_{s}\right)$ is set to 25bar, and the following variables are registered:

- $y_{r e f}$ : PRMS signal.

- $x_{v}$ : input signal of servo-valve.

- $P_{A}$ and $P_{B}$ : pressure at the piston's chambers $\mathrm{A}$ and $\mathrm{B}$, respectively.

- $F$ : force generated by the actuator.

- $y$ : position of the rod.

The signals captured with analog sensors (i.e. $P_{A}, P_{B}$ and $F$ ), are filtered with a 4 th order Butterworth low pass filter with $f_{c}=400[h z]$. 


\subsubsection{Closed Loop Transfer Function Identification}

For the first step of the identification, it is assumed that the open loop system is given by the following third order type-I system:

$$
\begin{aligned}
G(s) & =\frac{\operatorname{num} G(s)}{\operatorname{den} G(s)} \\
& =\frac{k w_{n}^{2}}{s\left(s^{2}+2 \zeta w_{n}+w_{n}^{2}\right)},
\end{aligned}
$$

therefore, the input-output behavior in close loop will be given by:

$$
\begin{aligned}
G_{c l}(s) & =\frac{K G(s)}{1+K G(s)}, \\
& =\frac{\operatorname{Knum} G(s)}{\operatorname{den} G(s)+\operatorname{Knum} G(s)} .
\end{aligned}
$$

For all the input-output data (i.e. $y_{r e f}$ and $y$ ) from the experiment, a third order model is determined implementing an ARMAX structure from the identification toolbox of Matlabß(93).

The identified models correspond to the close-loop system $G_{c l}(s)$. However, since the proportional controller $K$ is known, the open loop transfer function can be found, and it is given by:

$$
G_{o l}(s)=\frac{1}{K} \frac{n u m G_{c l}(s)}{\operatorname{den} G_{c l}(s)-n u m G_{c l}(s)}
$$

As it is common with models with poles at the origin, the identified models may present additional zeros and poles. Therefore, this inaccuracy must be corrected by selecting the poles and zeros that governs the dynamics. The new corrected model $G(s)$ is again validated with the experimental data.

\subsubsection{Dynamic Motion Parameters Identification}

Based on the linearized expression:

$$
A_{p} \delta P_{L}=m \delta \ddot{y}+\sigma \delta \dot{y}+\delta F_{e}
$$

the following linear system $\boldsymbol{A} \boldsymbol{x}=\boldsymbol{B}$ is defined:

$$
\left[\begin{array}{ccc}
\ddot{y}(1) & \dot{y}(1) & 1 \\
\ddot{y}(2) & \dot{y}(1) & 1 \\
\vdots & \vdots & \vdots \\
\ddot{y}(n) & \dot{y}(n) & 1
\end{array}\right]\left[\begin{array}{c}
m \\
\sigma \\
F_{e}
\end{array}\right]=\left[\begin{array}{c}
A_{p} P_{L}(1) \\
A_{p} P_{L}(2) \\
\vdots \\
A_{p} P_{L}(n)
\end{array}\right] .
$$




\section{EXPERIMENTAL IDENTIFICATION OF SHA}

The linear system (5.7) is solved implementing the least squares method provided with the lsqr function of Matlabß.

The parameters obtained are fitted to a second order polynomial dependent of the position, i.e. $\tilde{m}(y), \tilde{\sigma}(y), \delta \tilde{F}_{e}(y)$.

\subsubsection{Sensibilities Constants Optimization}

From and using the parameters of $G(s)$ and $\tilde{m}(y), \tilde{\sigma}(y), \delta \tilde{F}_{e}(y)$, the sensibilities constants can be found as follows:

$$
\begin{aligned}
K_{p} & =-b_{1}^{\prime}+\frac{\sigma}{\tilde{m}} \\
K_{d} & =\frac{1}{A_{p}}\left(\tilde{m} b_{0}^{\prime}+\tilde{\sigma} K_{p}\right) \\
K_{x} & =\frac{1}{A_{p}}\left(\tilde{m} b_{0}^{\prime} k_{01}\right)
\end{aligned}
$$

The complete linearized model is generated

$$
Y=\frac{k_{01} b_{0}^{\prime}}{s\left(s^{2}+b_{1}^{\prime} s+b_{0}^{\prime}\right)} X_{v}+\frac{k_{02} b_{0}^{\prime}\left(s+z_{0}\right)}{s\left(s^{2}+b_{1}^{\prime} s+b_{0}^{\prime}\right)} F_{e}
$$

Using the input-output data of the experiment, the sensibilities constants $\left[K_{x}, K_{d}, K_{p}\right]$ are modified in order to maximize the accuracy of the model, defining the following fitting index:

$$
F=1-\frac{\left\|\left(y-y_{s}\right)\right\|}{\|(y-\bar{y})\|}
$$

where $y$ is the output data from the experiment, and $y_{s}$ is the simulated output data with the transfer function.

\subsection{Experimental Setup}

The experimental setup is basically composed of an steel structure anchored at the floor that supports a stainless steel linear guide. The cylinder is attached to the structure and the cylinder's rod is attached to the sliding element of the linear guide in order to provide a linear movement (see Fig. 5.2).

Two pressure sensors are placed between the output ports of the servo-valve and the input ports of the cylinder, providing the pressure from the respective cylinder's chambers. 


\subsection{Experimental Setup}

A dSPACE DS1103 board is implemented as the control hardware. It captures data from the pressure sensors $\left(P_{A}\right.$ and $\left.P_{B}\right)$, force sensor $(F)$ and linear encoder $(y)$, it also provides the control signal $\left(x_{v}\right)$ to the servo-valve.

The experiment is fully designed in the Simulink®block diagram environment, and by means of the Real-Time Interface (RTI), it is embedded in the controller board. An interface developed with the dSPACE interface developer provides real time full control on the experiment and data visualization.

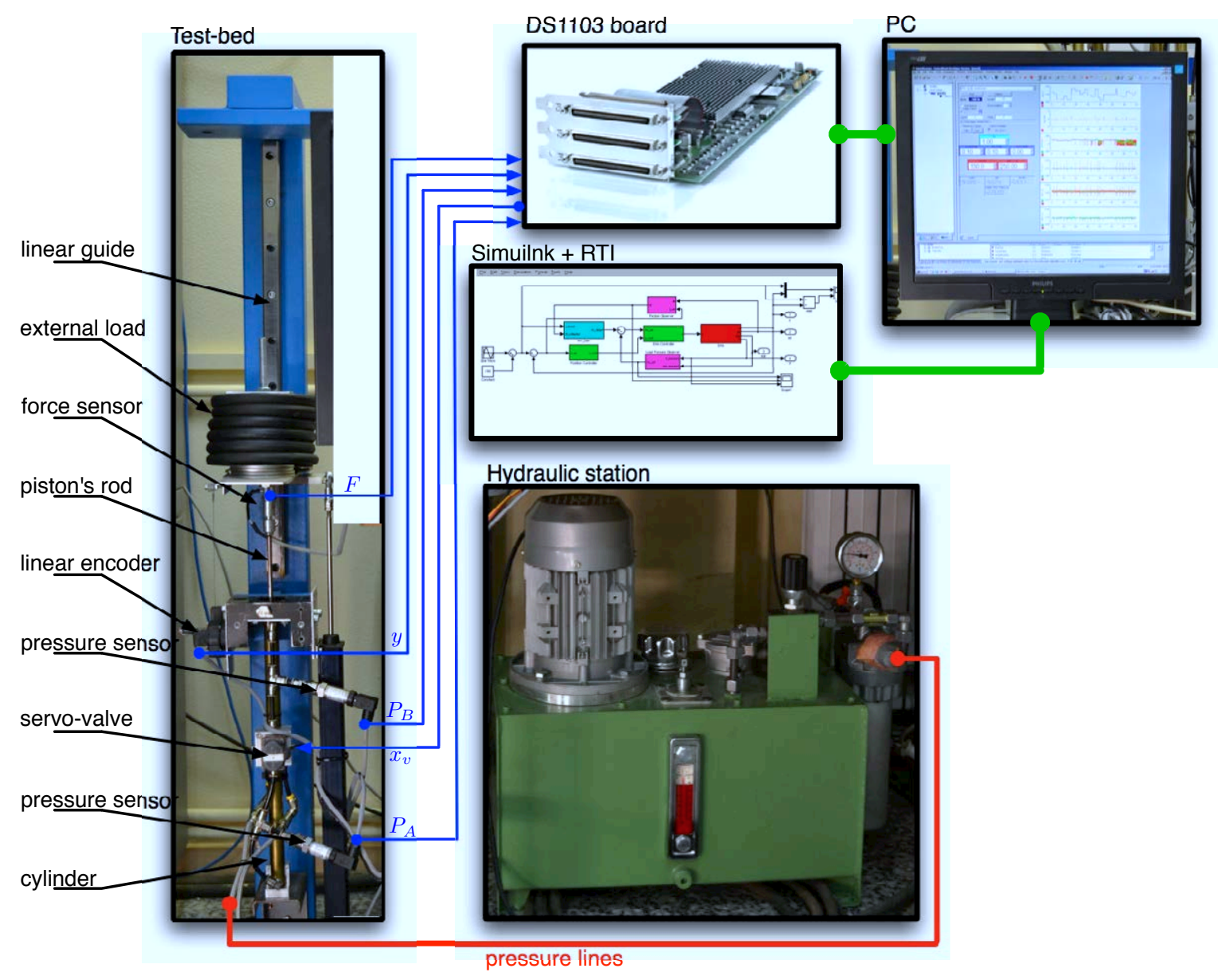

Figure 5.2: Experimental Setup. 


\subsection{Experimental identification}

\subsubsection{Experiment \#1: PRBS response under closed loop}

\section{- Objective}

Finding the parameters that characterizes the behavior of the hydraulic actuator for each set point and external load.

\section{- Description}

The SHA is submitted to PRMS input signals in closed loop, driving external loads of 0,3 and $6[\mathrm{Kg}]$. A proportional position controller is implemented. The range of work of the SHA is divided into 11 set points, separated by $30 \mathrm{~mm}$, except for the first and last set-point that are defined at $10 \mathrm{~mm}$ and $290 \mathrm{~mm}$ respectively. The PRMS signal provides a pseudorandom signal of $\pm 10 \mathrm{~mm}$ of amplitude centered at the set-point established. The period of the PRMS ranges from 1 to 6 seconds. Register of 50s are obtained. For each position and load, five different PRMS signals are used, with different proportional controller.

\section{- Data Processing}

- For the First ID step, it is only considered the transient response of the system. Therefore, each change in the PRMS signal is detected and a window of $150 \mathrm{~ms}$ is used in this step of the identification.

- $\delta P_{L}$ is calculated from $P_{A}$ and $P_{B}$, as follows: $\delta P_{L}=P_{A}-\alpha P_{B}$.

- $\dot{y}$ and $\ddot{y}$ are obtained implementing finite difference on $y$, and a $4 t h$ order Butterworth low-pass filter with a cut off frequency of $200[\mathrm{~Hz}]$.

\section{- Results}

1. In Fig. 5.3 it is presented in blue a typical PRMS signal used during the experimental identification and the position response of the system in red. The curves correspond to an experiment for a set point of $10 \mathrm{~mm}$ with non external load (i.e. $0[\mathrm{Kg}]$ ). As it can be observed, the response presents overshoot and decaying oscillations which were intentionally looked for during the selection of the proportional gain of the controller in order to get all the dynamics as possible without falling into instability. 


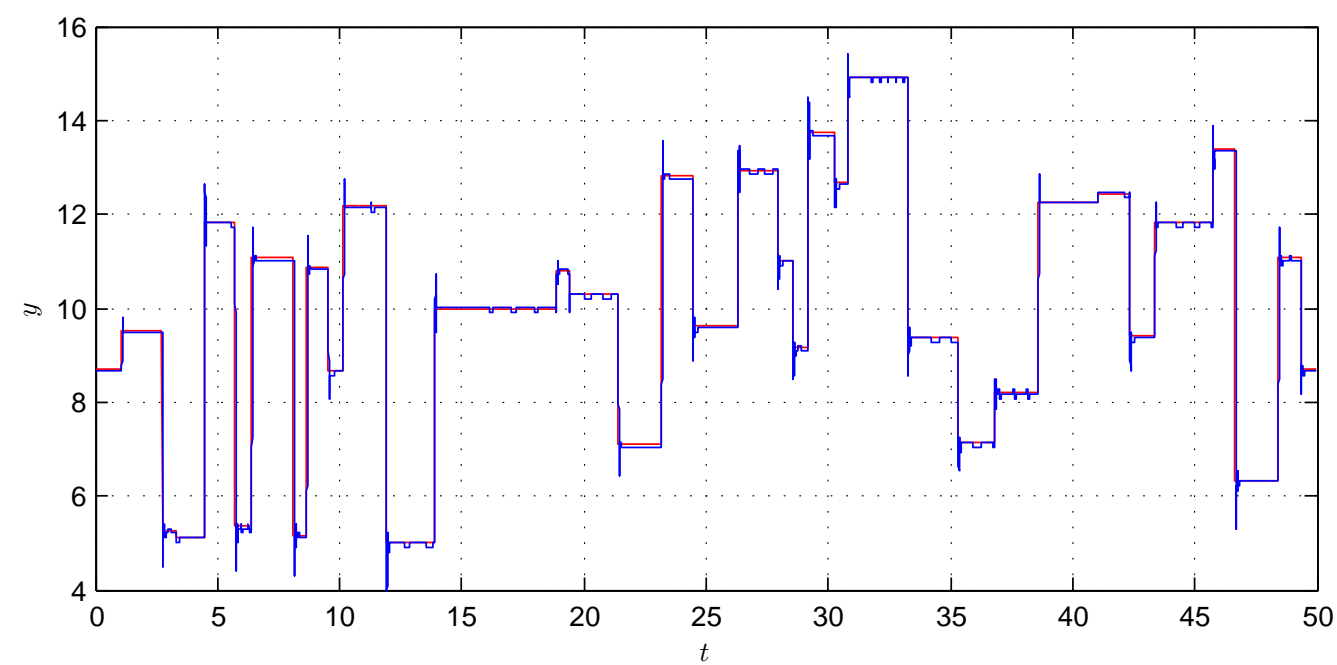

Figure 5.3: Input-output experimental data. PRBS input signal (i.e. $u_{r e f}$ ) in blue, pistons response in red.

2. For each register, more than 25 identified sets of parameters associated to the transient response of the cylinder's motion dynamics were found. A typical result from a single register from an experiment with an external load of $0[\mathrm{Kg}]$ is presented in Fig. 5.4. As it can be observed, the identified parameters for each transient response (dots in red) present a variability for each set point. However, a global behavior along all the set points can also be observed. Evaluating, several fitting criteria, is was found that the identified parameter are best fitted to a quadratic polynomial (dots in blue). On the other hand, it is possible to appreciate the linear relation between the mass and the external perturbation. This is caused by the effect of the gravity $(g)$ since the experimentation is performed against it, thus the external perturbation is basically given by $F_{e} \approx m g$.

3. After all the data is processed, and the identified parameter from the transient response are fitted with a quadratic polynomial, the parameters that govern the dynamics of the system are found (see Fig. 5.5). As it was expected, the parameters vary according the external load varies. The linear relationship between the mass and the external perturbation is maintained. It can also been seen that the quadratic behavior smoothes while increasing the external load. The parameters identified for the 33 working points are attached in the appendix in Table ??. 

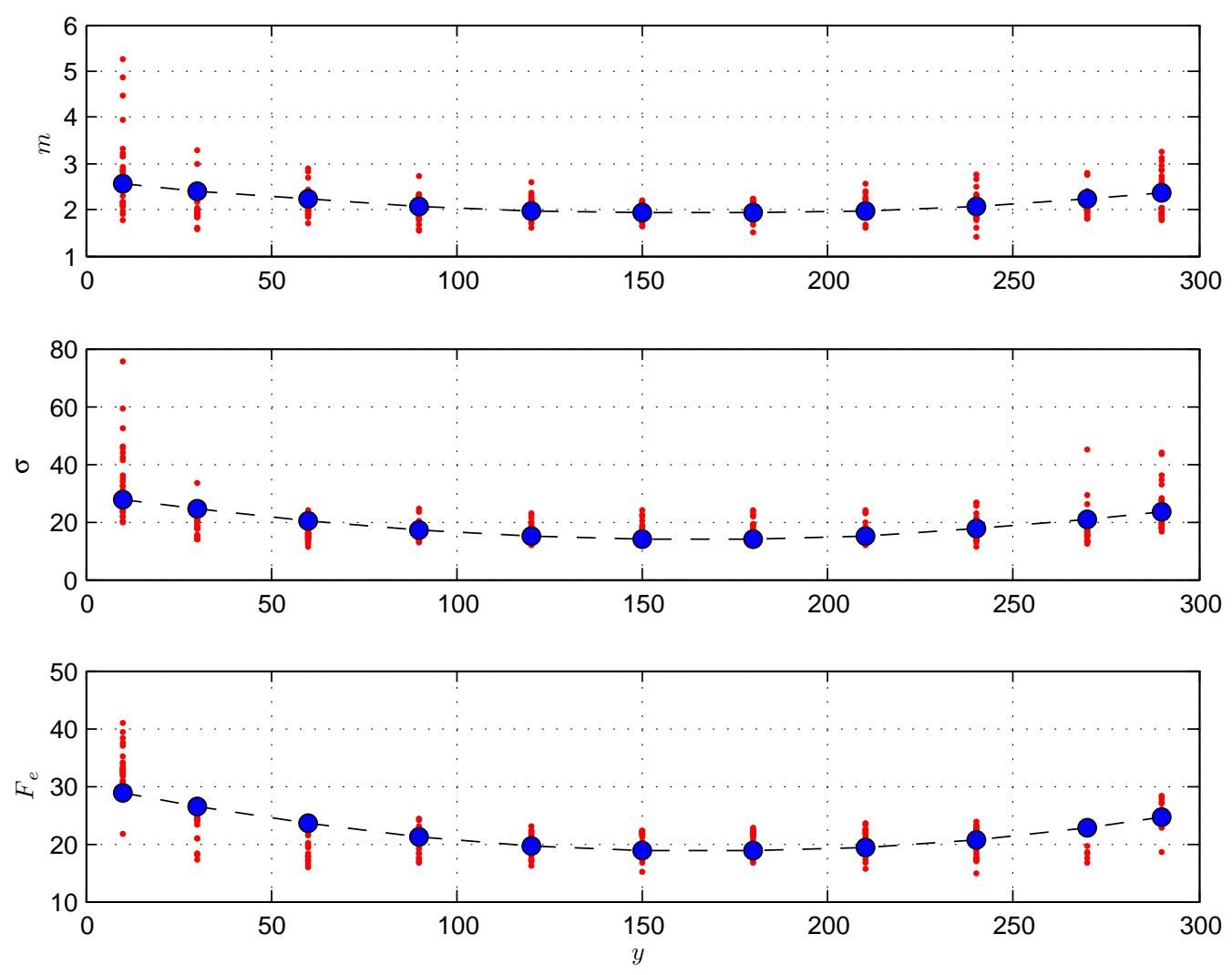

Figure 5.4: A typical result from the identified sets of parameter associated with the motions dynamics for no external load. The identified parameters for each transient response are in red, and in blue it is presented the a fit to a quadratic polynomial. $m$ is in $[\mathrm{Kg}], \sigma$ is in $[\mathrm{Ns} / \mathrm{mm}], F_{e}$ is in $[\mathrm{N}]$ and $y$ is in $[\mathrm{mm}]$.

4. From the input-output data (i.e. $P R M S$ and $y$ ), a close-loop transfer function $G_{c l}(s)$ is found for every transient response. For each set point and load it is selected the $G_{c l}(s)$ that maximizes (5.12) for all the registers. Using (5.5), the open loop transfers functions $G_{o l}$ are found. In Fig. 5.6, it is presented the pole-zero diagram of all the open loop function transfers for and external load of $0 \mathrm{Kg}$. In this picture, it can be observed that the identified models are conformed by a polynomial of fifth order in the denominator and a fourth order for the numerator. The increase of the order of the polynomial while the ARMAX structure is estimating is typical when the system to be identified posses a pole near the origin. However, it can be seen that the there exists zeros and poles that are very close, thus they can be cancelled. In the same way, the poles and zeros that are located away of the dominant 

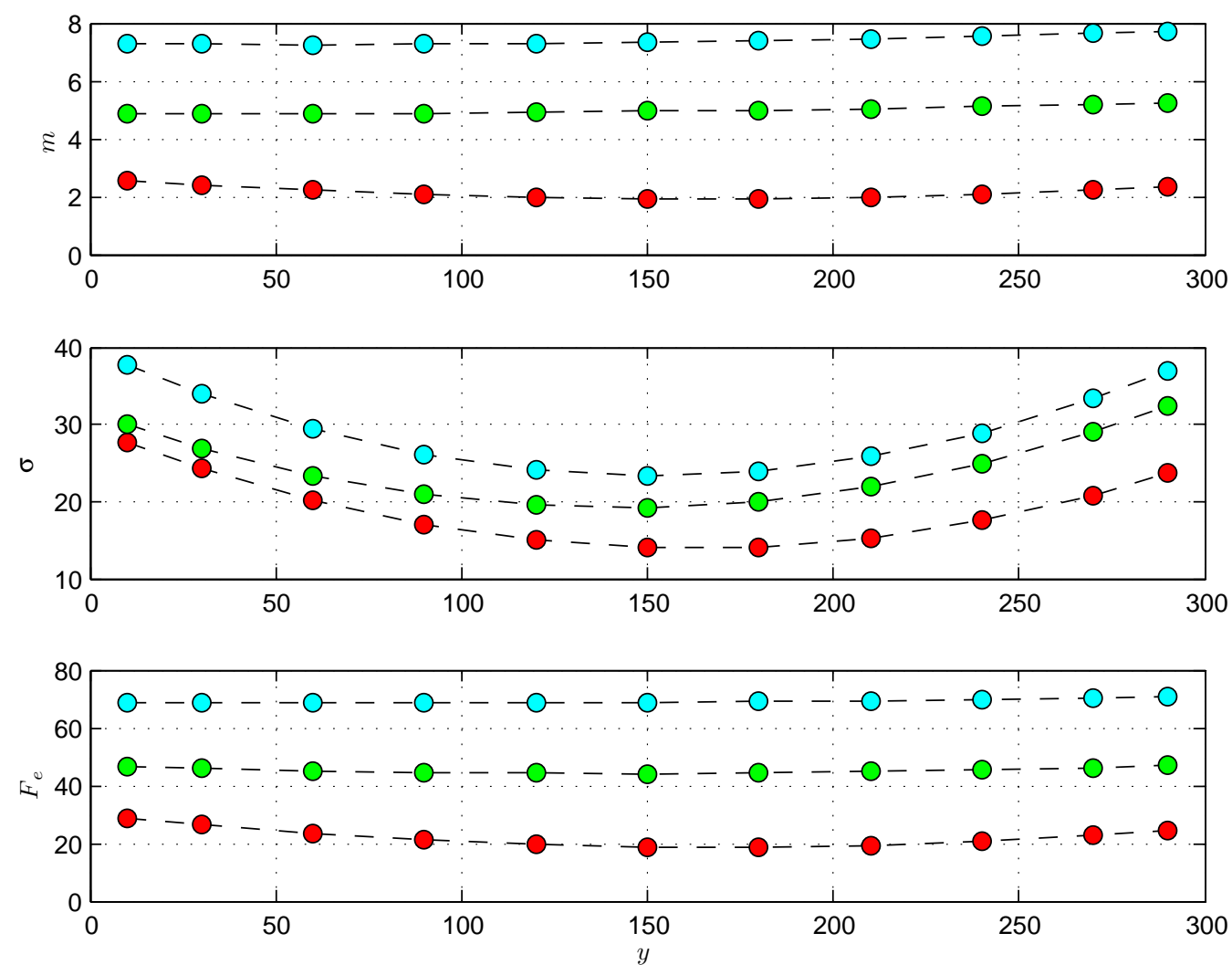

Figure 5.5: Parameters of the system with $0 \mathrm{Kg}$ (red), $3 \mathrm{Kg}$ (cyan) and $6 \mathrm{Kg}$ (green) of external load.

dynamics (see Fig. 5.6(b)) of the system can be also neglected. It is also observed that the identified models do not have poles exactly at the origin, instead they are very close to it. Therefore, this inaccuracy is neglected and the poles are forced to be at the origin.

5. The sensibility constants $K_{d}, K_{p}$ and $K_{x}$ are first approached using the the parameters $\tilde{m}, \tilde{\sigma}, \tilde{F}_{e}$ and the transfer function $G(s)$. Then, they are modified in order to maximize (5.12) using the input-output data from the experiment and the complete linearized model (5.11). The optimized sensibility constants are presented in Fig. 5.7 . As it can be observed, they depend both on the piston's position and the external load, and they present a quadratic behavior. The sensibility constants are summed up in the appendix (see Table ??).

6. The complete linearized model is obtained from the parameters identified, 


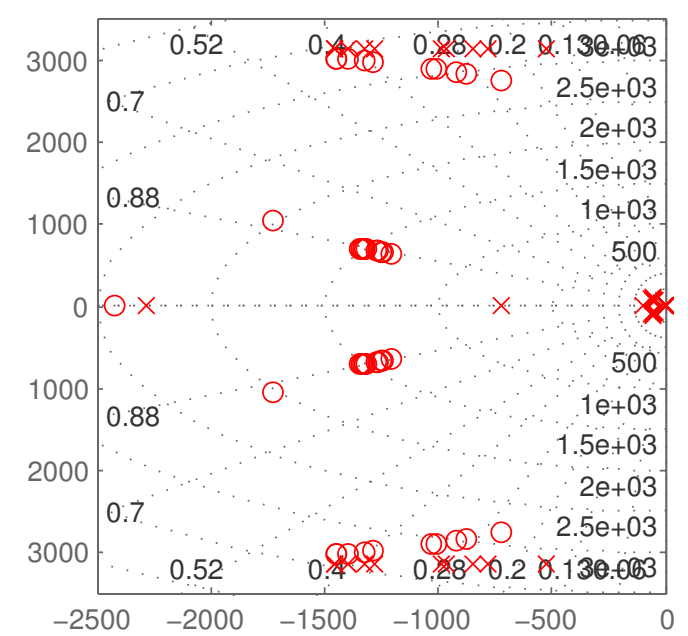

(a)

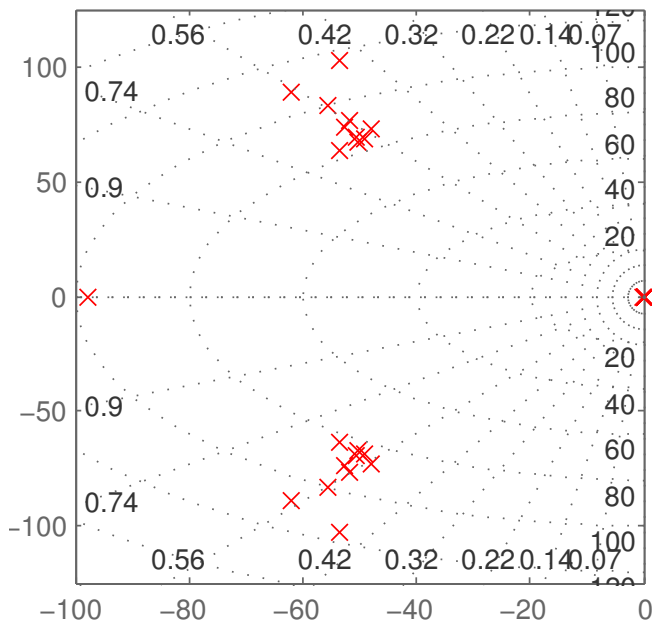

(b)

Figure 5.6: $G_{o l}$ pole-zero diagram. 5.6(a): complete model. 5.6(b):governing dynamics.

and the pole-zero diagram of $P(s)=\Delta Y / \Delta X$ is presented in Fig. 5.8(a). As it can be seen, the systems presents the typical pair of conjugate complex poles with a pole at the origin. It can be observed, that the location of the complex pair varies with the external load. Furthermore, the complex pair tends to the origin as the external load increases. As it was expected, the location of the complex pair also varies with piston's position (see Fig. 5.8(b)). The location of the complex pair varies inside the interval $[-65,-45] s^{-1}$ for the real part, and $[75,125] \mathrm{s}^{-1}$ for the imaginary part.

7. All the identified systems are validated with the input-output data. The accuracy of the identified models are given by the ability to reproduce the output data for a given input data, and it is quantified with (5.12). The accuracy of the systems identified are among the $85 \%$ and $94 \%$. In Fig. 5.9 it is presented the response of the identified system and the real output data. As it can be observed, the simulated response with the identified model it is almost the same than the real output data.

8. In Fig.5.10 is presented the resulting Simulink model of the HSA. As it can be observed, the model is constructed with the set of parameters of the transfer function identified for each working point given in Table ?? presented in the appendix. The transition from one working point to another is performed 

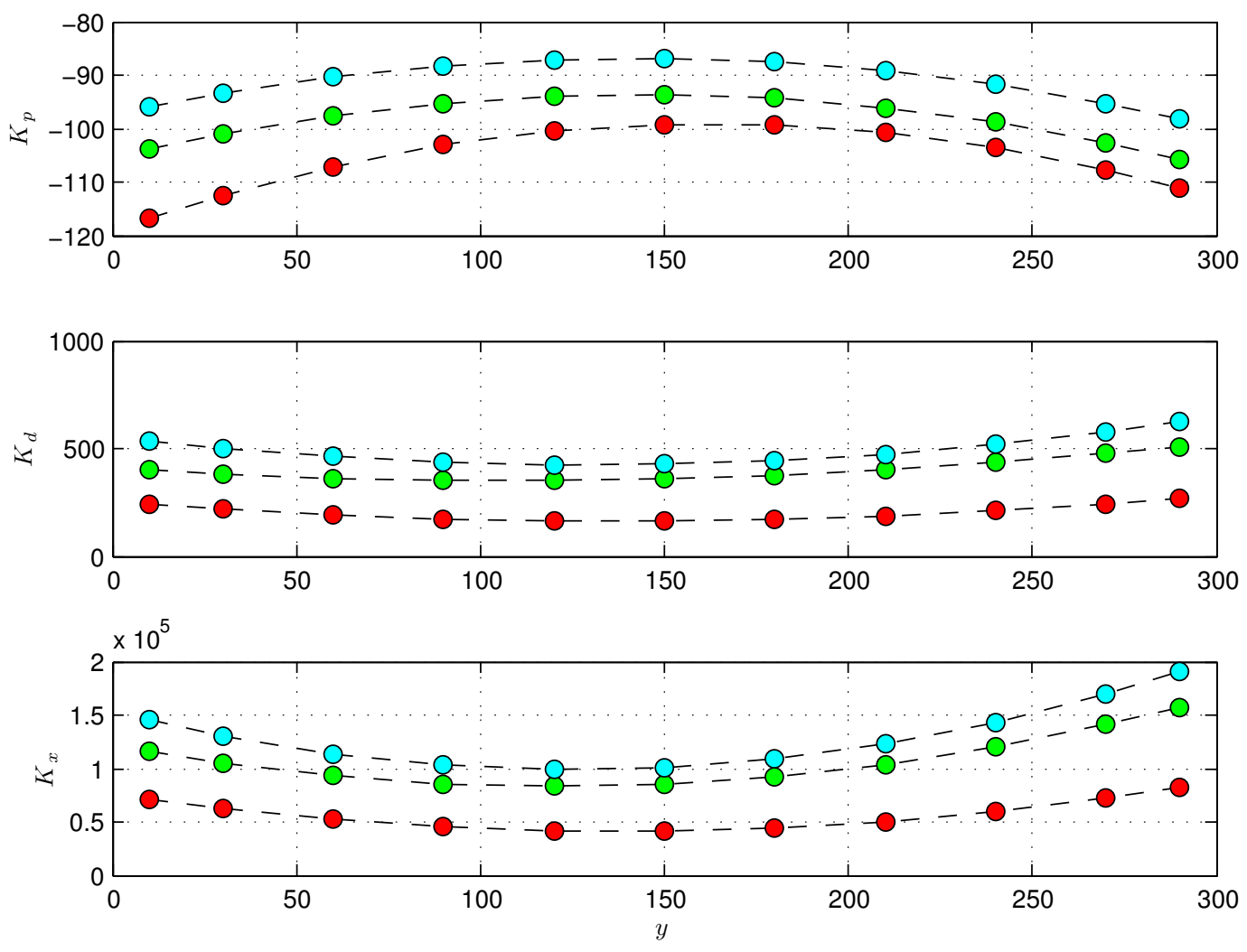

Figure 5.7: Identified sensibility constants. $0 \mathrm{Kg}$ (red), $3 \mathrm{Kg}$ (cyan) and $6 \mathrm{Kg}$ (green) of external load.

using the spline interpolation characteristic of the 2D table of Simulink block. Fig.

\subsection{Conclusions}

The identification process requires a deep knowledge of the system under analysis. The process is not straight forward and practical expertise with the system helps to define and adopt logical criteria, e.g. the mass of the system can not be negative, that simplifies the process. Nevertheless, it is an iterative process that requires intuition to get the most appropriate results.

The system under analysis is a velocity system, i.e. for a given constant input the HSA provides a movement with constant velocity. Therefore, to identify a linearized model for a given working point, the identification process must be performed in a 


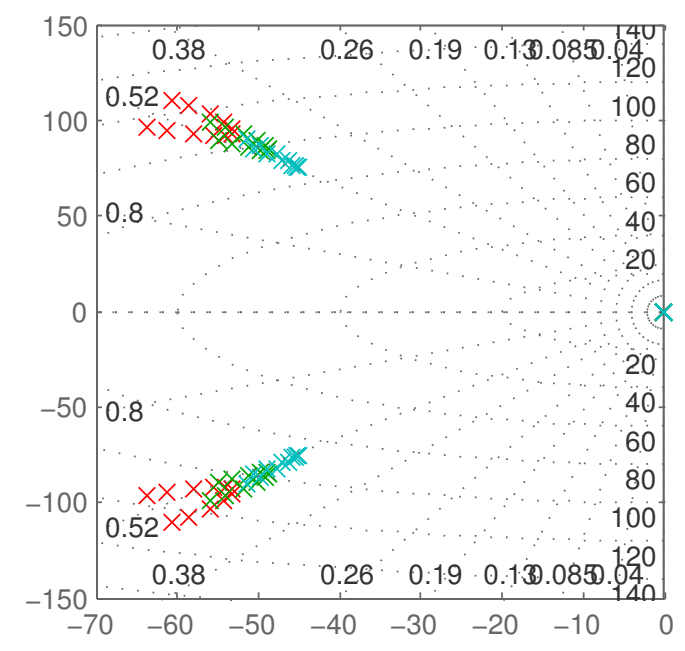

(a)

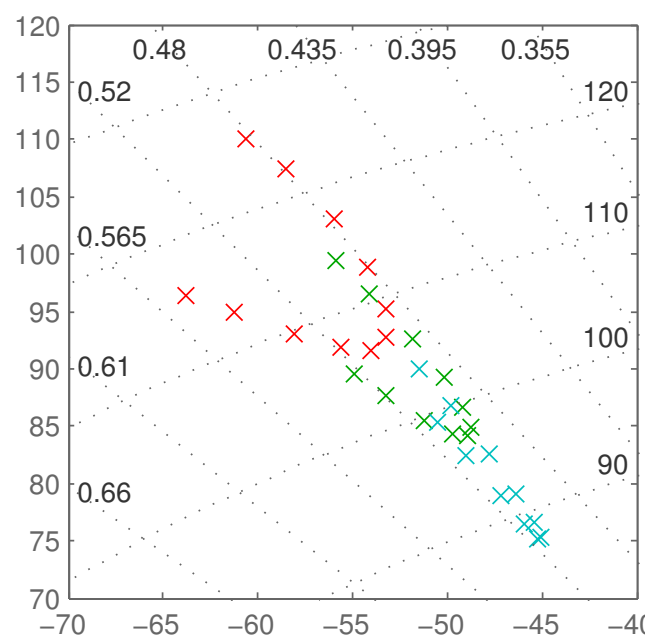

(b)

Figure 5.8: Pole-zero diagram of $P(s)=\Delta Y / \Delta X$. 5.6(a): complete model. 5.6(b): detail of the behavior of the complex pair. $0 \mathrm{Kg}$ (red), $3 \mathrm{Kg}$ (cyan) and $6 \mathrm{Kg}$ (green) of external load.

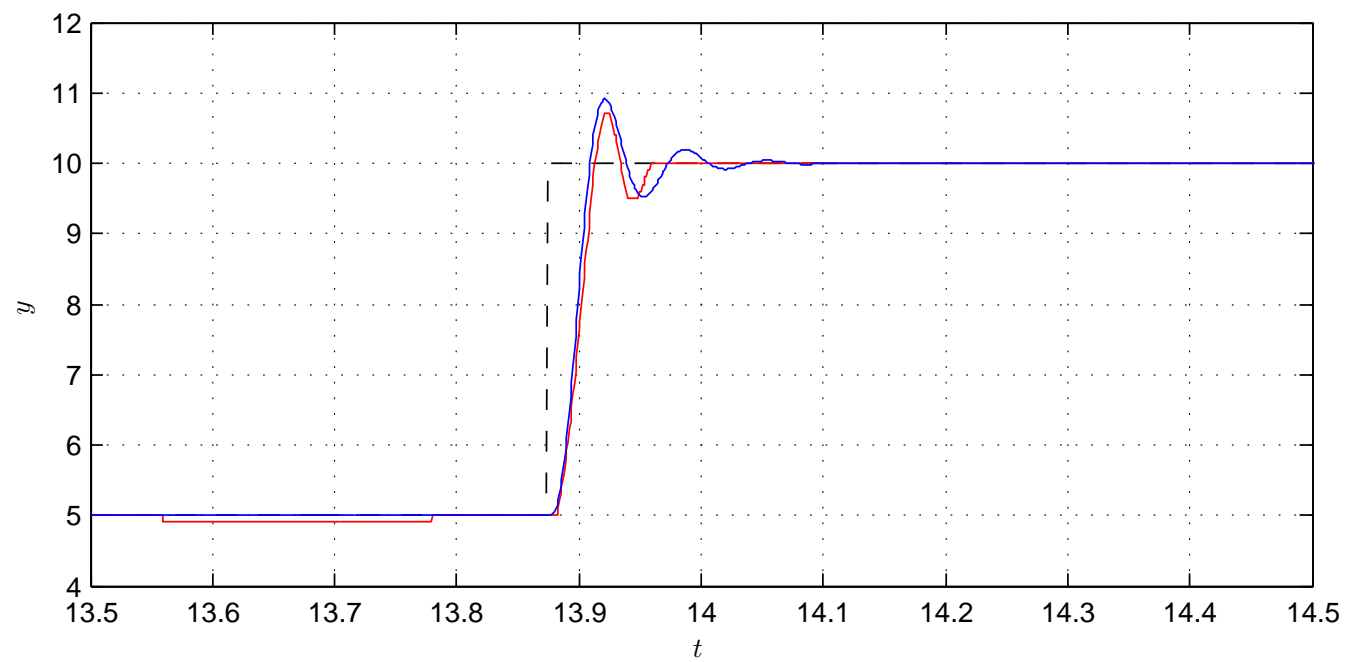

Figure 5.9: Simulated response of the identified model. In dashed black is the PRMS signal, in red the real output data and in blue the simulated response of the identified model.

closed loop with a position controller.

The localization of the pair of complex pole on the complex plane, reveal that the 


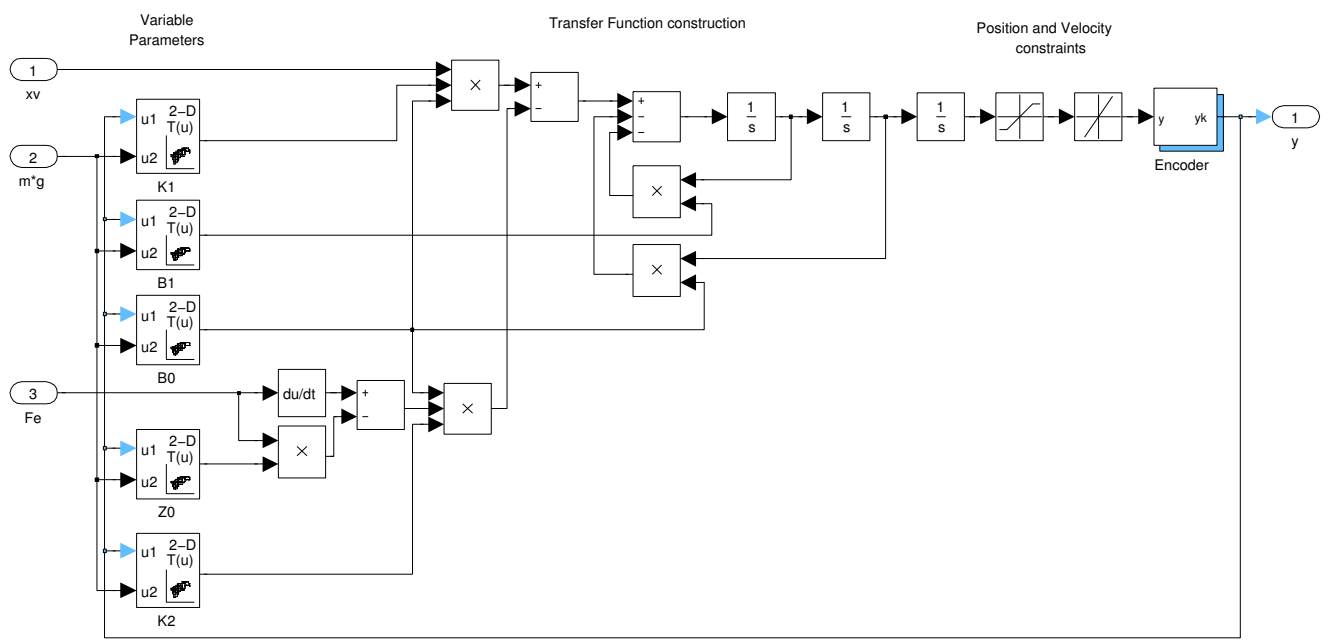

Figure 5.10: Simulink model of the identified HSA

system is more stiff according the external load arises. It is also seen that when the piston approaches to the mid of the maximum stroke localization of the complex poles get nearer the imaginary axis of the complex plane, and thus presenting the worst stability margins (149). 
5. EXPERIMENTAL IDENTIFICATION OF SHA 


\title{
Experimental Friction Model Identification
}

\author{
""... we are good lost now... \\ For certain, you have to be lost \\ to find the place that can not be found... \\ Else way, everybody will know where it was..." \\ Captain Barbosa.
}

\subsection{Introduction}

Friction appear as reaction forces at the physical interface between two surface in contact, which are the results of many different mechanisms that depend on contact geometry and topology, material of the bodies, relative motion and presence of lubrication (15). In a linear hydraulic actuator, the rods and the seals, and between the piston o-rings, seals, and cylinder, and the viscous effects of the hydraulic fluid all generate friction.

It is an inevitable non-linear phenomenon that occurs in all kinds of mechanical system, hence it plays a major role in control systems and it is generally an impediment for servo control resulting in steady state errors and instabilities due to the appearance of limit cycles (28), (38),(117), (112). 


\section{EXPERIMENTAL FRICTION MODEL IDENTIFICATION}

In order to deal with this problem, there are two different approaches. The first one, is to try to diminish these effects by designing the system and/or operate in regions where the nonlinear behavior are minimum. As in (116) where an extra motor is added in order to provide a permanent rotational velocity to a linear actuator avoiding the Stribeck region between the rod and the piston. The other alternative, is to identified the effect and compensate it within the control loop. This last approach requires of the understanding of the effect and further modeling it.

The study of friction forces dates back to the observations of Leonardo Da Vinci, who introduced the concept of the existence of a force that is proportional to load, opposes the motion, and is independent of contact. Since then, many models based on experimental observation have been proposed. A review of frictions models can be found in (114) (19), and a discussion of the properties of some dynamical models are presented in (14), physics-motivated models and empirically motivated models for friction's dynamics are presented and discussed in (2).

In this chapter is presented the frictions effects observed in the SHA, and the experimental identification of the parameters of the LuGre model. The parameters are found by means of two different experiments. The first one is performed with motions at constant velocity and the second one is performed under controlled force. These experiments allow to find separately the set of parameters that govern the steady state and the pre-sliding regime, respectively.

\subsection{Friction effects observed in the SHA}

\subsubsection{Oscillations - Limit cycles}

In Fig. 6.1 is presented a suddenly burst of oscillation of the SHA at low velocity motion under controlled force excitation. The curve at the top of the figure shows the sinusoidal excitation with an amplitude of $10[\mathrm{~N}]$ at $0.04[\mathrm{~Hz}]$ applied to the SHA. As it can be observed, the force exerted by the piston (middle curve) falls into oscillations at $t \approx 17$ [s], and the position of the SHA decays.

Taking a closer look at the curves (see Fig. 6.2), it can be seen that the amplitude of the oscillations enlarges until it reaches a given amplitude and frequency (curve at the top). Oscillations are also present in the position of the SHA (curve at the bottom). 

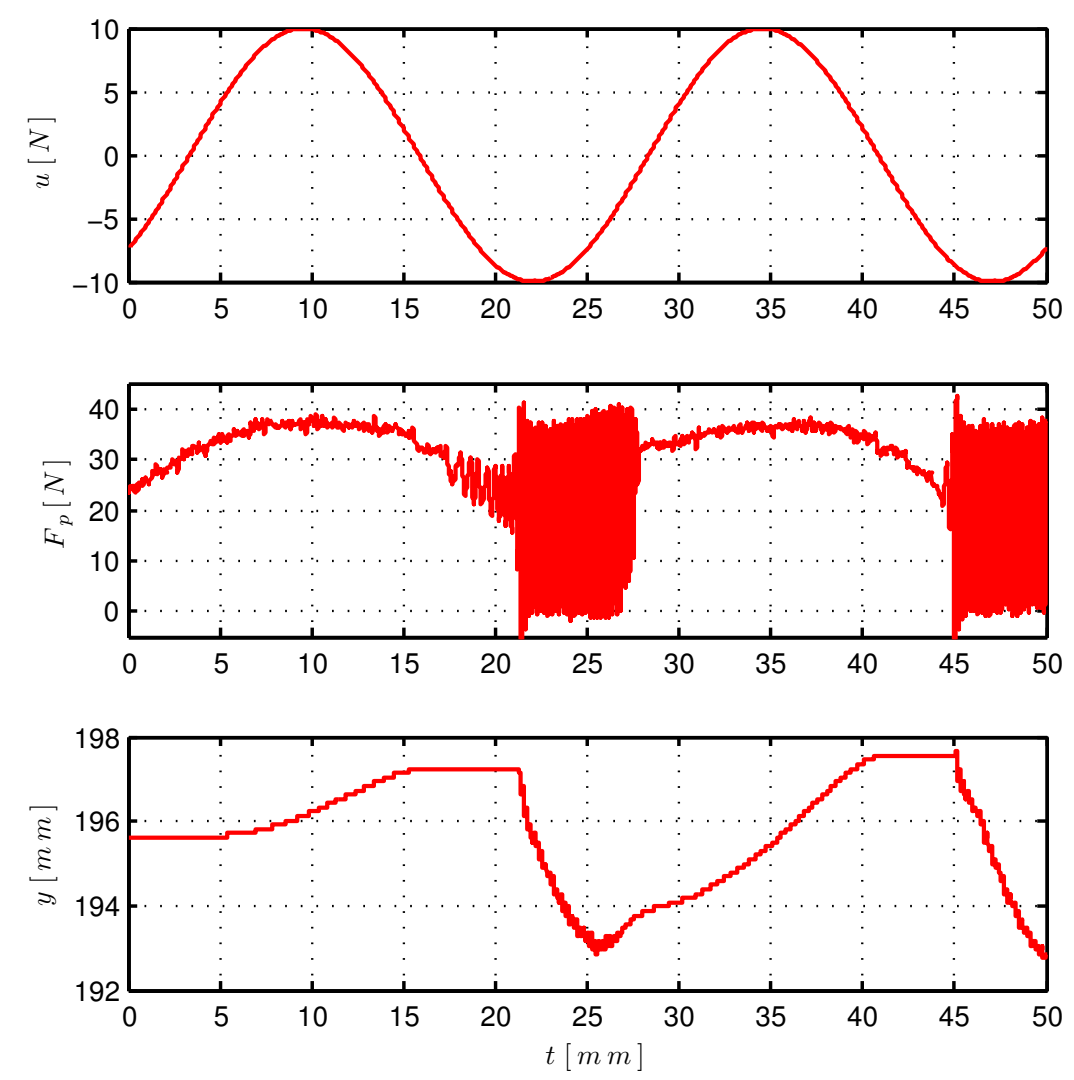

Figure 6.1: Oscillations caused by friction.

This particular behavior, named stick-slip motion is caused by the friction force at zero velocity, which is higher than at a small nonzero velocity. When motion starts, the friction force decreases rapidly and the accelerating force is therefore large. The motion is jerky and switches between periods of sticking and slipping, resulting in poor control performance, and can also give rise to vibrations and noise (113).

\subsection{LuGre Model Friction}

Despite the persistent efforts of many researchers around the globe, there is still no model that captures all of the experimentally observed aspects of friction into one formulation. However, the LuGre model represents a good compromise between simplicity and reproduction of complex friction behavior. It has few parameters and represents a good choice to express friction at zero velocity and gross motion. 


\section{EXPERIMENTAL FRICTION MODEL IDENTIFICATION}
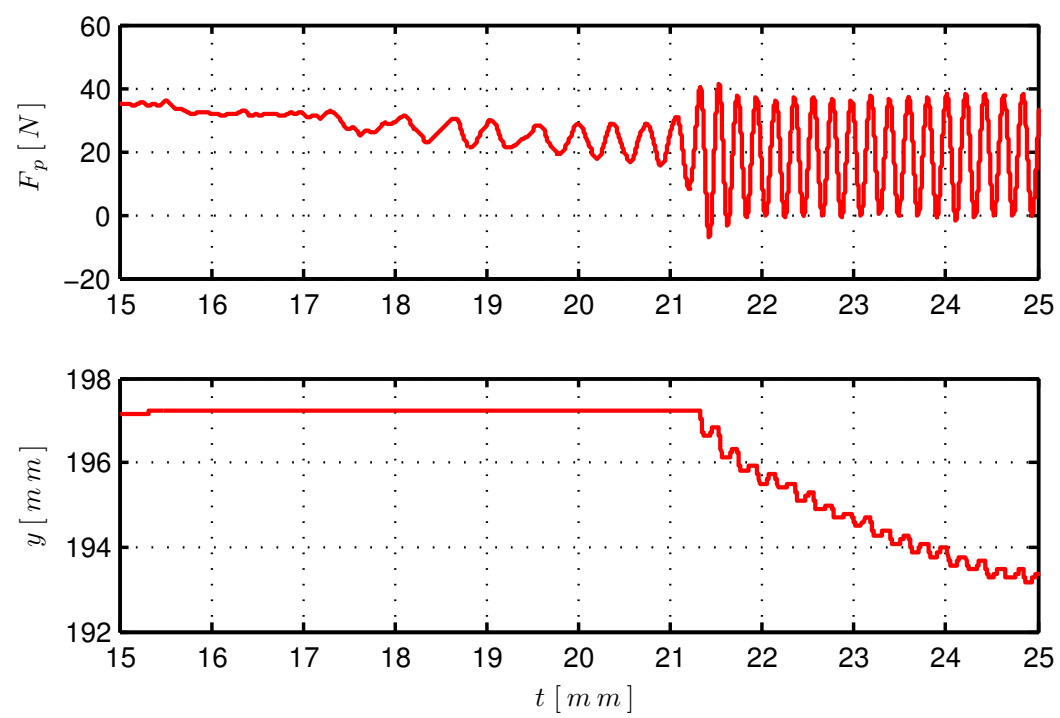

Figure 6.2: Detail of the oscillations.

The LuGre model (37), is an extension of the dynamic Dahl model, but it captures the Stribeck effect and describes the stick-slip motion. More over, the LuGre model has passivity properties that are useful for designing friction compensator that give asymptotically stable closed-loop systems. A comprehensive description of the model and its properties can be found in (113).

The standard LuGre model is described by the following parametrization:

$$
\begin{aligned}
F_{f} & =\sigma_{0} z+\sigma_{1} \frac{d z}{d t}+\sigma_{2} \dot{y} \\
\frac{d z}{d t} & =\dot{y}-\sigma_{0} \frac{|\dot{y}|}{g(\dot{y})} z \\
g(\dot{y}) & =\alpha_{0}+\alpha_{1} e^{-\left(\dot{y} / v_{s}\right)^{2}}
\end{aligned}
$$

where $F_{f}$ is the friction force, $\dot{y}$ is the relative velocity between two the surface in contact, and $z$ is the internal friction state that can be interpreted as the average bristle deflection. The LuGre model reproduces the spring-like behavior for small displacement, where $\sigma_{0}$ is the stiffness and $\sigma_{1}$ is the microdamping. The macrodamping (i.e viscous friction) is govern by $\sigma_{2}$. The velocity dependent function $g(\dot{y})$, provides the Coulomb friction $\left(\alpha_{0}\right)$ and Stribeck effect $\left(\alpha_{1}\right.$ and $\left.v_{s}\right)$. 


\subsection{Experimental Identification of LuGre Model Friction}

The parameters that compose the dynamical model can be found experimentally by considering the different regimes of friction and the set of parameter associated to them (64) (10). Therefore, the two step off-line identification methodology presented in (89) is implemented in this work. First, constant velocity tests are performed to identify the static velocity to friction map. In a second step, stick-slip motions are performed and a simplified friction model is used in order to identify the two dynamic parameters.

\subsubsection{Steady State Regime}

At constant velocity, it is considered that the interaction between the contact surfaces are in steady state regime, therefore the micro-damping effect can be neglected ( i.e. $\dot{z}=0)$ and (6.1) is reduced to the classic static friction model:

$$
F_{s s}(\dot{y})=\alpha_{0}+\alpha_{1} e^{-\dot{y} / v_{s}^{2}}+\sigma_{2} \dot{y}
$$

Hence, the static velocity to friction map, can be obtained by establishing a series of constant velocity settings of the piston and, at each setting, measuring the friction force.

Given the integrating behavior with regard to the position of the cylinder, constant velocity motions can be achieved by providing steps inputs to the servo-valve of the SHA in open loop.

\subsubsection{Experiment \#1: Constant Velocity Motions}

\section{- Objective}

Finding the parameters that govern the behavior of friction in steady state regime: $\alpha_{0}, \alpha_{1}, \sigma_{2}, v_{s}$.

\section{- Description of the experiment}

The SHA is submitted to step inputs in open loop driving external loads of 0 , 3 and $6[\mathrm{Kg}]$. More than 50 tests with different step intensities in the range of $\left[-u_{\min }, u_{\max }\right]$ of the servo-valve for each external load are performed.

\section{- Data processing}




\section{EXPERIMENTAL FRICTION MODEL IDENTIFICATION}

- The velocity and acceleration of the piston are obtained from its position by means of $1 s t$ and $2 n d$ order finite difference, respectively. The signals are filtered with a $4 t h$ order Butterworth low-pass filter with a cut off frequency at $200[\mathrm{~Hz}]$.

- The friction force can not be directly measured, therefore it is found from the SHA's dynamic equation of motion (4.15) as follows:

$$
F_{f p}=F_{P}-m_{T} \ddot{y}(t)-m_{T} g-F_{f g},
$$

where $F_{P}$ is the force exerted by the piston given by: $F_{p}=0.1 A_{p}\left(P_{A}-\right.$ $\left.\alpha P_{B}\right), P_{A}$ and $P_{B}$ are the pressure at the chambers $\mathrm{A}$ and $\mathrm{B}$ of the piston, respectively. $m_{T}$ is the external load, $F_{f g}$ is the friction force of the linear guide, $\ddot{y}$ is the piston's acceleration and $g$ is the constant of gravity.

- It is assumed that the transient response to step input can be neglected after $0.5 \mathrm{~s}$. Hence, the static velocity to friction map is generated with the set of data of $F_{f p}(t)$ and $\dot{y}(t)$ that belongs to $t \epsilon[0.5,1] s$.

- The parameters of $F_{s s}(\dot{y})$ are estimated by solving a non linear curve-fitting problem in least-squares sense implementing the the lsqcurvefit function of Matlabß). All the values of $F_{f p}$ and $\dot{y}$ obtained during the tests are gathered into two matrices and used as input data of this function. Lower and upper bounds for each parameters are defined according to the maximum and minimum values observed during the tests.

\section{- Results}

1. A typical step response of the SHA in open loop is presented in Fig. 6.3. As it can be observed, a constant voltage at the input of the servo-valve produces a motion with constant velocity. The velocity curve, shows that transients can be neglected for $t>0.5 \mathrm{~s}$. For the interval $0.5 \mathrm{~s} \leqslant t \leqslant 1 \mathrm{~s}$, it can be considered that $F_{f p}(t)$ and $\dot{y}(t)$ remain constant.

2. The static velocity to friction map of the SHA for different external loads is depicted in Fig.6.4(a). The data sets are plotted as little grey dots and the mean of the data sets are plotted as coloured markers. As it can be observed, the mapping presents an asymmetrical behavior and it is more sensible to external load changes in motions with negative velocities. Furthermore, for gross motion with positive velocities (i.e. $\dot{y}>v_{s}$ ), it can be said that there is 

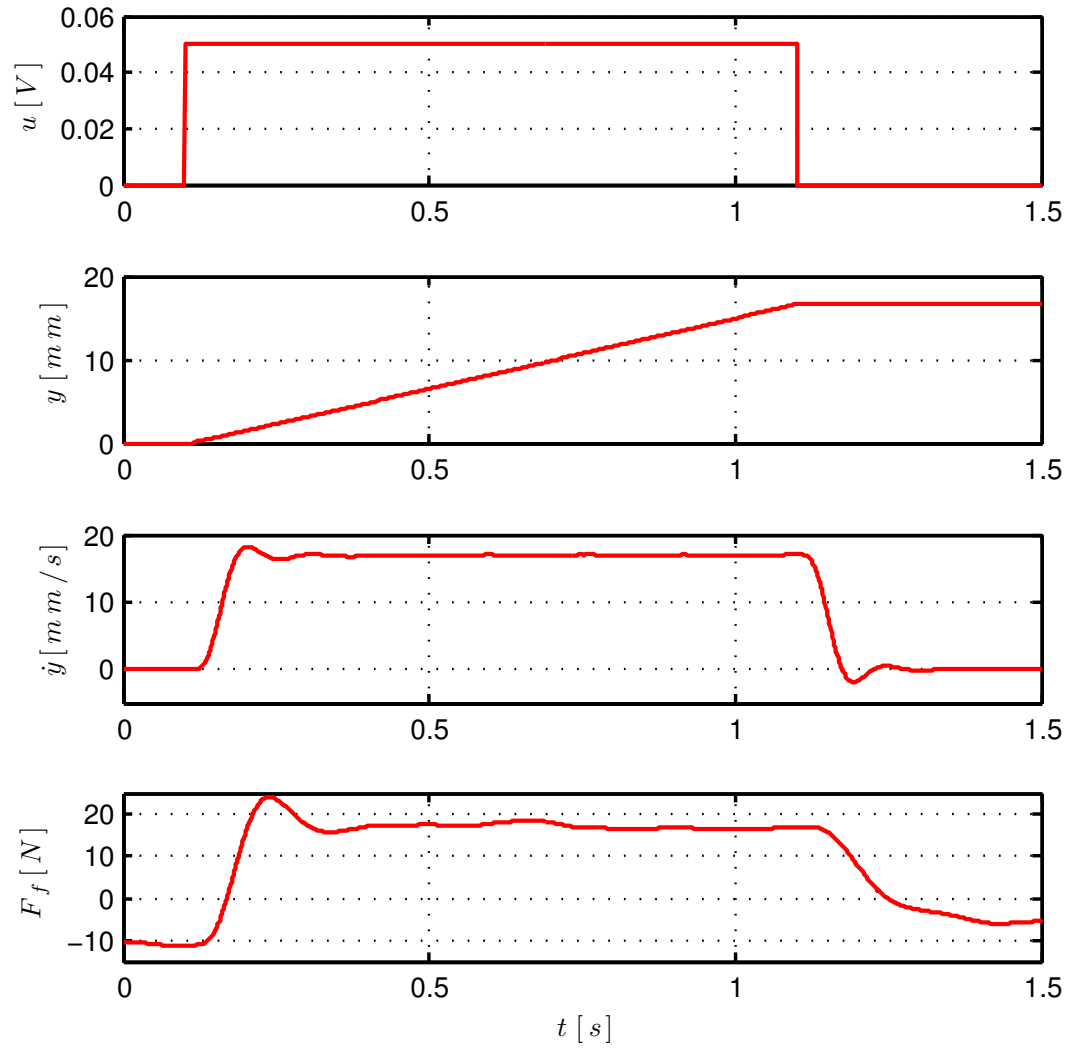

Figure 6.3: Step response in open loop.

no significant difference between the curves for different loads, which is not the case for $\dot{y}<0$. However, it can also be observed that there is a tendency of the curves to converge to a line for $\dot{y}<-150[\mathrm{~mm} / \mathrm{s}]$.

3. The non linear curve fitting of the data obtained during the tests to the friction model (6.4) is presented in Fig. 6.4(b), superposed to the experimental data. It can be observed that the fitted curve, preserves the asymmetrical characteristic of the curves found during experimentation.

4. The parameters of the friction model in steady regime are summed up in Table 6.1.

\subsubsection{Pre-sliding regime}

In pre-sliding regime the friction force is due to elastic and plastic deformations of the asperity contacts. Therefore, the interface between two contact surfaces can be viewed 


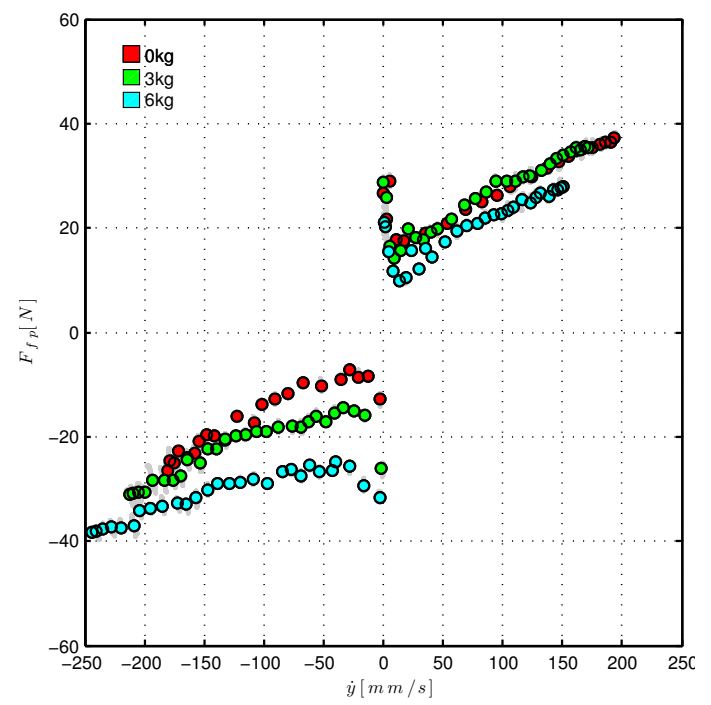

(a)

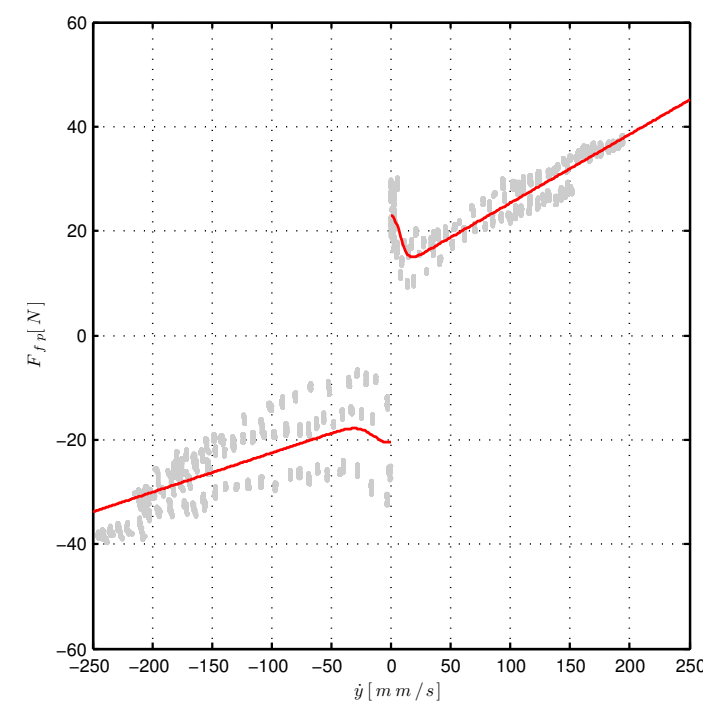

(b)

Figure 6.4: Friction force characteristic of the SHA in steady state regime for different external loads.

Table 6.1: Estimated parameters for the friction model in steady state regime.

\begin{tabular}{cll} 
Parameter & Value & Unit \\
\hline \hline$\alpha_{0}{ }^{+}$ & 12.1994 & {$[\mathrm{~N}]$} \\
$\alpha_{1}{ }^{+}$ & 10.6468 & {$[\mathrm{~N}]$} \\
$v_{s}{ }^{+}$ & 10.0000 & {$[\mathrm{~mm} / \mathrm{s}]$} \\
$\sigma_{2}{ }^{+}$ & 0.1316 & {$[\mathrm{Ns} / \mathrm{mm}]$} \\
\hline$\alpha_{0}{ }^{-}$ & -15.0000 & {$[\mathrm{~N}]$} \\
$\alpha_{1}{ }^{-}$ & -5.3903 & {$[\mathrm{~N}]$} \\
$v_{s}{ }^{-}$ & -20 & {$[\mathrm{~mm} / \mathrm{s}]$} \\
$\sigma_{2}{ }^{-}$ & 0.0753 & {$[\mathrm{Ns} / \mathrm{mm}]$} \\
\hline
\end{tabular}

as an interface formed by springs. As a force is applied the springs are extended which results in the friction force. If the extension becomes too large the springs snap and sliding occurs.

Therefore, the pre-sliding regime is given by motions at very low velocity (i.e. $\dot{y} \approx$ 0 ), produced by an external force $F$ below the static friction force $F_{s}$ (i.e. $F<F_{s}$ ). 
Then, linearizing (6.1) for $z=0$ and $\dot{y}=0$, the friction model is approached to (see (113) for the detailed maths and assumptions considered for the linearization):

$$
\begin{aligned}
\delta F_{f} & =\sigma_{0} \delta z+\left(\sigma_{0}+\sigma_{1}\right) \delta \dot{y}, \\
\frac{d}{d t}[\delta z] & =\delta \dot{y} .
\end{aligned}
$$

Considering that $z \approx 0$ and $\dot{y} \approx 0$, it can be assumed that $\dot{y} \approx \dot{z}$, then:

$$
F_{f} \approx \sigma_{0} y+\left(\sigma_{1}+\sigma_{2}\right) \dot{y}
$$

Hence, the elastic characteristic of friction can be explored experimentally, by applying an external force $F$ smaller than the stiction force to the SHA at rest, and measuring the piston's displacement.

\subsubsection{Experiment \#2: Force Control Response}

\section{- Objective}

Finding the parameters that define the pre-sliding behavior of the friction of the SHA: $\sigma_{0}$ and $\sigma_{1}$.

\section{- Description}

The SHA is submitted to controlled force tests, providing small motions at very low velocities. Controlled force is achieved by feeding back the force exerted by the piston (i.e. $F_{p}$ ) and implementing a proportional controller. A sinusoid input at $0.04[\mathrm{~Hz}]$, with 30 different amplitudes below static friction is applied to the SHA. Tests for loads of 0,3 , and $6[\mathrm{Kg}]$ are performed.

\section{- Data Processing}

- The velocity and acceleration of the piston are obtained from its position by means of 1 st and $2 n d$ order finite difference, respectively. The signals are filtered with a $4 t h$ order Butterworth low-pass filter with a cut off frequency at $10[\mathrm{~Hz}]$.

- The friction force is obtained according to (6.5).

- Since, the resolution of the position sensor is not precise enough, the numerical identification of (6.8) can not be processed. Instead, a graphical estimation is performed. Therefore, in all the tests, it is detected the forces 


\section{EXPERIMENTAL FRICTION MODEL IDENTIFICATION}

that produce the minimum distance that the position sensor is capable of detect (i.e. $0.1[\mathrm{~mm}]$ ), and the corresponding force-displacement chart is generated.

- It is assumed that at small displacement the linearized friction model can be simplified into:

$$
F_{f} \approx \sigma_{0} y,
$$

therefore, at low velocities friction is govern by $\sigma_{0}$ and it is obtained graphically as the slope of the force in the force-displacement chart.

- The effects of the parameter $\sigma_{1}$ are critical at micro-scale, however in mechanical systems where the sensor resolutions are within the millimeter scale, the effect of $\sigma_{1}$ are minor, and its main role is to damp the linearized equation in the pre-sliding regime rather than to finely match the data (17). Therefore, let us replace (6.8) in (4.15), and the motion's dynamic of the SHA in pre-sliding regime results into:

$$
m_{T} \ddot{y}=u-\left(\sigma_{0} y+\left(\sigma_{1}+\sigma_{2}\right) \dot{y}\right),
$$

where $u=F_{p}-m_{T} g$. Let us now define the linear approximated map $G(s): y \rightarrow u$ as follows:

$$
G(s)=\frac{1}{m_{T} s^{2}+s\left(\sigma_{1}+\sigma_{2}\right)+\sigma_{0}} .
$$

In order to obtained a well-behaved stick-slip transition, it is imposed a damping ration $\zeta=1$ for (6.11). Therefore, $\sigma_{1}$ can be obtained as follows:

$$
\sigma_{1}=2 \zeta \sqrt{\sigma_{0} m_{T}}-\sigma_{2}
$$

\section{- Results}

1. In Fig. 6.5 is presented the curves obtained during one of the tests carried on with an external load of $6 \mathrm{Kg}$. A set point of $60[\mathrm{~N}]$ is stablished in order to counteract the weight of the external load. From this result it can be observed that the response in position does not present the same behavior in upward and downward displacement. It can be observed that in upward displacement there is a bigger component that opposes the motion. This result is clearly depicted in the friction force presented in the last curve. As it can be seen, the friction force rises upto almost $30[N]$ in the positive direction of motion, but it hardly reaches the $-10[N]$ in the opposite direction. 

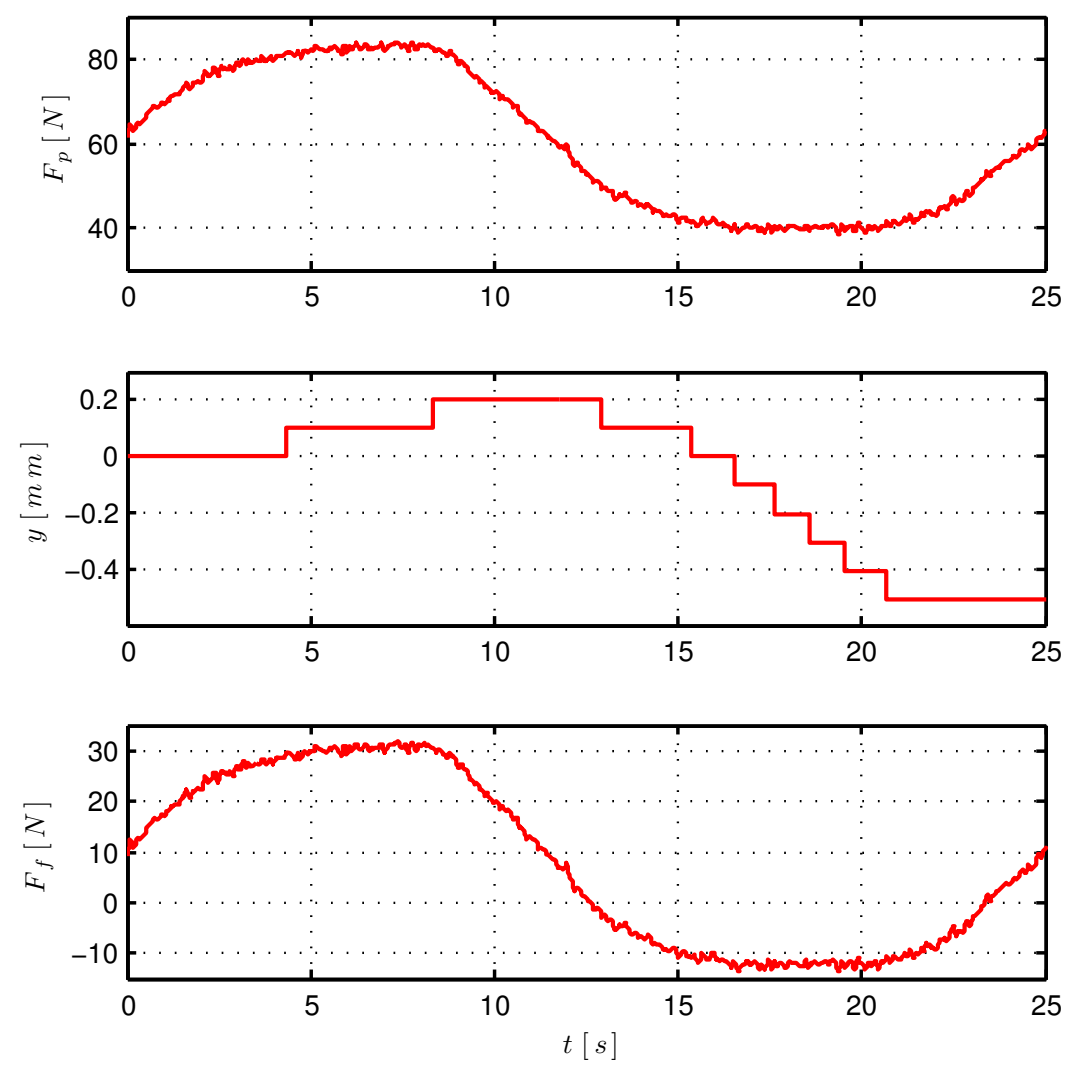

Figure 6.5: Curves obtained from a force control test. Top: force generated exerted by the piston. Middle: position response. Bottom: calculated friction force.

2. The results of the tests are condensed in the chart graph presented in Fig. 6.6(a). As it can be seen, the elastic property of the pre-sliding regime conserves its characteristic even though the tests are carried on with different external loads. The asymmetry of the position response to force excursions can be also observed.

3. The linear fitting to the slope of the Force-displacement chart graph is presented in Fig. 6.6(b), remarking the asymmetry in the slope for positive and negative motions.

4. The parameters that govern the pre-sliding regime are summed up in Table 6.2. The parameter $\sigma_{1}$, was calculated considering an external load of $3[\mathrm{Kg}]$, in order to guaranty passivity of the model, i.e. $\zeta<\frac{\sigma_{2}}{2 \sqrt{\sigma_{0} m_{T}}}\left(\frac{\alpha_{0}}{\alpha_{1}}+1\right)$ (see 


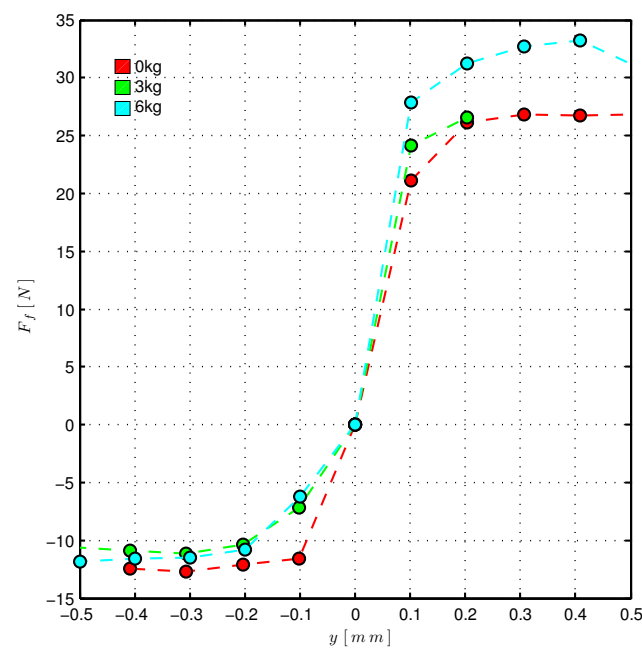

(a)

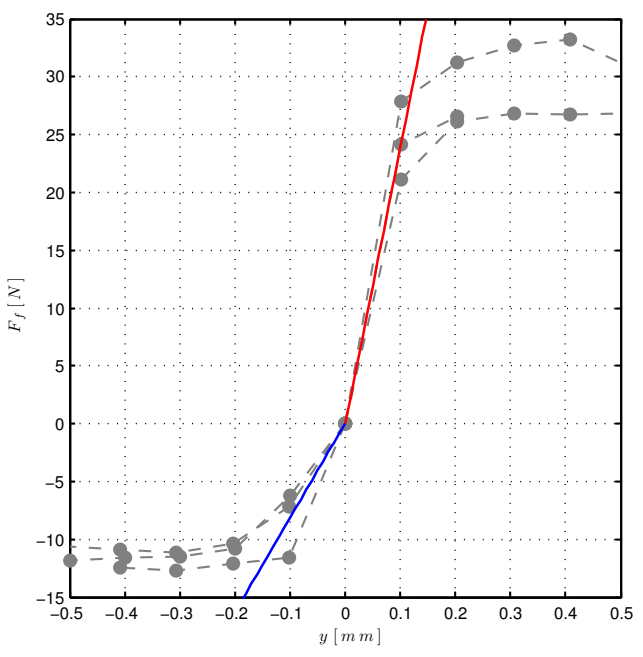

(b)

Figure 6.6: Force-displacement chart graph in pre-sliding regime.

(17),(23) for further details).

Table 6.2: Pre-sliding parameters estimation.

\begin{tabular}{ccc} 
Parameter & Value & Unit \\
\hline \hline$\sigma_{0}{ }^{+}$ & 238.9709 & $\mathrm{~N} / \mathrm{mm}$ \\
$\sigma_{1}{ }^{+}$ & 1.8944 & $\mathrm{Ns} / \mathrm{mm}$ \\
$\sigma_{0}{ }^{-}$ & 81.5808 & $\mathrm{~N} / \mathrm{mm}$ \\
$\sigma_{1}{ }^{-}$ & 1.0911 & $\mathrm{Ns} / \mathrm{mm}$ \\
\hline
\end{tabular}

\subsection{Conclusions}

Friction effects are perhaps one of the most undesirable nonlinearities always present in any dynamic system, leading to instability, limit cycles and offset error.

Friction has several states and regimes that depends of the materials and relative velocity of the contact surface. The models found in the literature are as complex as the regimes that they pretend to recreate. 
One of the critical regimes of friction is the stick-slip effect which are generated in very low velocity. This effect gives origin to limit cycles.

Therefore, for precision control it is needed a model that could represent this effects, such as the Lu-Gre model, that provides almost all the characteristics and regimes of friction: static friction, stick-slip, streabeck effect, viscous friction.

The identification of this model, can only be performed by steps, and identifying each of the parameters associated to each regime. 
6. EXPERIMENTAL FRICTION MODEL IDENTIFICATION 


\section{7 \\ Control Strategies SHA}

“... being brilliant is not enough... you have to work hard... Intelligence is not a privilege is a gift, and you use it for the man kind ..."

Doctor Otto Octavius

\subsection{Introduction}

As it was shown in previous chapters, the dynamics of hydraulic systems are highly nonlinear due to the physical properties present in the system such as fluid compressibility, pressure losses, transient and turbulent flow conditions and non-linear friction characteristics in the hydraulic actuator.

In addition to these nonlinear characteristics which often demand the development of advanced control schemes, model uncertainty is a challenge for high performance hydraulic control problems.

As a consequence, control of hydraulic systems is always a challenge.

In order to achieve high precision control performance, advanced control schemes which consider the real dynamic characteristics of the hydraulic system (nonlinear actuator friction, compressibility of the hydraulic oil, hysteresis, and sensor disturbance) are desirable for many applications. 


\section{CONTROL STRATEGIES SHA}

Precise control of electro-hydraulic actuated systems, due to their inherent nonlinear characteristics, cannot be easily obtained with conventional linear controllers.

In this chapter it is presented two control strategies for the position control of the SHA. The first one is a classical PI controller, assuming that the controller must with stand all the external perturbation, whose stability and performance is demonstrated to be robust. The second one, is a more complex controller that considers in its design information of the external perturbation, such as the gravity, and friction forces and its own dynamical variability.

\subsection{Control Strategies implemented in the SHA}

In the literature can be found several approaches for the control of linear SHA. In this section a summed up of the strategies implemented are presented.

Due to the fact that the SHA are highly used in the industry, the main strategy used for position control is the classic PID, which have proved to be reliable in bounded regimes and most of all due to the fact that are easily implemented by the practitioner.

There are several approaches for the PID design(18), however, due the nonlinear inherent characteristic of an hydraulic system, a fixed PID can not provide a good control performance in all situations. Therefore, another criteria must be adopted for the selection of the PID. In (91), it is presented an optimum tunning for the non linear PID controller, considering the desired time response of a second order system. In (139) is introduced a technique in order to provide a robust PID tunning.

Fuzzy controllers have been identified as promising alternatives to conventional controllers and have been proven to achieve a better performance over linear controllers. In (134), is introduced a fuzzy PD based controller, with an integral and offset rules that are activated only in the fuzzy zero velocity error region in order to provide zero steady estate error for servo valve with dead-band.

In (155), a novel strategy for a full state feedback is implemented by defining membership functions for velocity, final position and stroke for different external loads. The control law is found using a product operator assuming known ending position for an unknown external load and stroke.

In order to overcome the problem of fixed gain PID controller, in (160) is presented a self tunning PID controller. The membership functions are defined for each of the 
parameter of the controller, whose range of work are firstly stated from and offline identified and simulated model.

On the other hand Sliding controller design provides a systematic approach to the problem of maintaining stability and consistent performance in the face of modeling imprecisions.

Sliding mode control (SMC) or also called variable structure, is a nonlinear control method, which provides a symmetric approach to the problem of maintaining stability and consistent performance in the face of modeling imprecision and disturbances. The sliding mode control possesses the following advantages: fast response, less sensitive to uncertainties, and easy implementation. However, sliding mode control relays on a discontinuous switching control, which may lead to chattering. The chattering control input has some drawbacks: easy damage of mechanism and excitation of unmodeled dynamics. Although the boundary layer method can attenuate the degree of highfrequency control input, its stability is guaranteed only outside the boundary layer, and its asymptotic tracking often cannot be achieve if the boundary layer is insufficiently small. One of the pioneer work on sliding control implemented for position control of a hydraulic servo is (92).

In (29), addresses the problem of position control of a SHA in presence of friction and compares its results with a classical PD. The control implements a sliding controller defining an surface based on the state error in position, velocity and acceleration. The friction observer is incorporated in the acceleration feedback loop. In order to reduce the chattering of the control action a Fuzzy technique is incorporated. In order to avoid the chattering produced by the switching function, a varying bounding layer is implemented in (41) for a position tracking, and in (125) for compensate friction forces and internal leakage.

Adaptive control is a valid method to overcome system uncertainties, especially uncertainties derived from uncertain parameters. (26) presents an adaptive controller for a hydraulic system which uses a feedforward with recursive least squares parameter estimation for the dynamic inversion of the system, and a feedback with a discretetime Riccati equation, providing high tracking performance. In (152) a discontinuous projection-based ARC controller is presented for high-performance robust control of electrohydraulic systems driven by single-rod actuators. Projection based adaptive law is also implemented in (103) (102). 


\section{CONTROL STRATEGIES SHA}

In (44) it is presented a position control for a hydraulic system based on a fuzzy logic learning algorithm that allows to enhance the accuracy of the model and thus providing adaptability to the changes in its dynamic. However, due to the computational costs it may present limitation for real-time implementation.

It is also common to combine control strategies, as in (61), where and adapting sliding controller is implemented on a SHA. The adaptation law compensates the nonlinear uncertain parameters caused by the varieties of the original control volumes, and the sliding mode control method is used to implement a novel-type Lyapunov function to construct an asymptotically stable controller.

Force and pressure tracking are important for some applications, such as vibration isolation and automotive active suspension, friction compensation among others.

Unlike electric actuators, force control of hydraulic actuators is a difficult problem, since in a hydraulic actuator, the control current acts to move the spool valve that controls the flow of hydraulic fluid into and out of the actuator. This flow in turn causes a pressure differential buildup that is proportional to the actuator force. Even if the spool valve dynamics are ignored, the control current fundamentally controls the derivative of the actuator force and not the force itself (see (4.4), (4.4), (4.13) and (4.14)), revealing an inherent lag of the system. A common strategy is the implementation of impedance control, i.e., produce a force in response to an imposed position. In (68) is presented a nonlinear PI position controller nested within a force feedback loop with second order target impedance.

In (7), it is shown using simple analytical tools, that basic PID controller can not provide force tracking and that it is necessary more advanced control strategies, such as $H_{\infty}$ (82), sliding controller (142), sliding-adaptive (5), predictive (151).

In (25) the sliding control strategy is implemented for the position control of a 1DoF manipulator. In this work it is implemented and state observer for the velocity and acceleration in order to suppress the chattering effects due to unmodelled highfrequency dynamics.

In general, in order to compensate the effects of friction, this last one has to be estimated and introduced into a force control loop with a feedforward scheme (6), (54), (79), (143).

Increasing the positioning accuracy in such systems requires adequate measures to alleviate the adverse effects of friction. One of the most common ways is to provide 
the controller with quantitative information on friction, achieving what is commonly referred to as model-based friction compensation. As direct measurement of friction is not possible, two options are models based on experimental friction identification or the use of nonlinear friction observers

A new sliding mode control with time-varying switching gain and a time-varying boundary layer has been introduced in (70) to modify the traditional sliding mode avoiding chattering control and ensuring asymptotic tracking. The sliding surface is defined in terms of the error position, its integral and the integral of the force error.

In (140) the inverse relation between the net fluid force of the cylinder and the input current to the servo-valve are used to define an appropriate Lyapunov function, providing force and position tracking with friction compensation. This strategy is further implemented in simulation of a climbing robot in (21). A simpler alternative to the work of (140) is presented In (138), where the outer-loop is replaced with a much simpler and robust scheme based on the application of the time-delay approach to approximate the dynamic inversion, providing better results than (140) and classic PID controllers.

Other nonlinear techniques, such as nonlinear backsteping (76), quantitative feedback (110) are also implemented.

\subsection{A brief discussion on the controller design}

As it was presented on the previous chapter, the linearized model of the SHA is given by:

$$
Y(s)=\frac{k_{1} b_{0}}{s\left(s^{2}+b_{1} s+b_{0}\right)} X_{v}(s)+\frac{k_{2} b_{0}\left(s+z_{0}\right)}{s\left(s^{2}+b_{1} s+b_{0}\right)} F_{e}(s),
$$

where the controlled variable is the position $Y(s)$ and the manipulated variable is the input current of the servo-valve $X_{v}(s) . F_{e}$, as it was presented on chapter 4.4, represents the effects of external forces, which in absent of external perturbations they are mainly due to the action of gravity. Then, without loosing generality, this variable could be considered as a perturbation, and the system could be simplified to:

$$
Y(s)=\frac{k_{1} b_{0}}{s\left(s^{2}+b_{1} s+b_{0}\right)} X_{v}(s) .
$$

Therefore, considering the parameters of the identified models presented in 5.4, classic P, PI, PD, PID are tuned by simulation and implemented in the real model for 


\section{CONTROL STRATEGIES SHA}

control position. The position time responses of the SHA with these controllers are presented in Fig. 7.1.

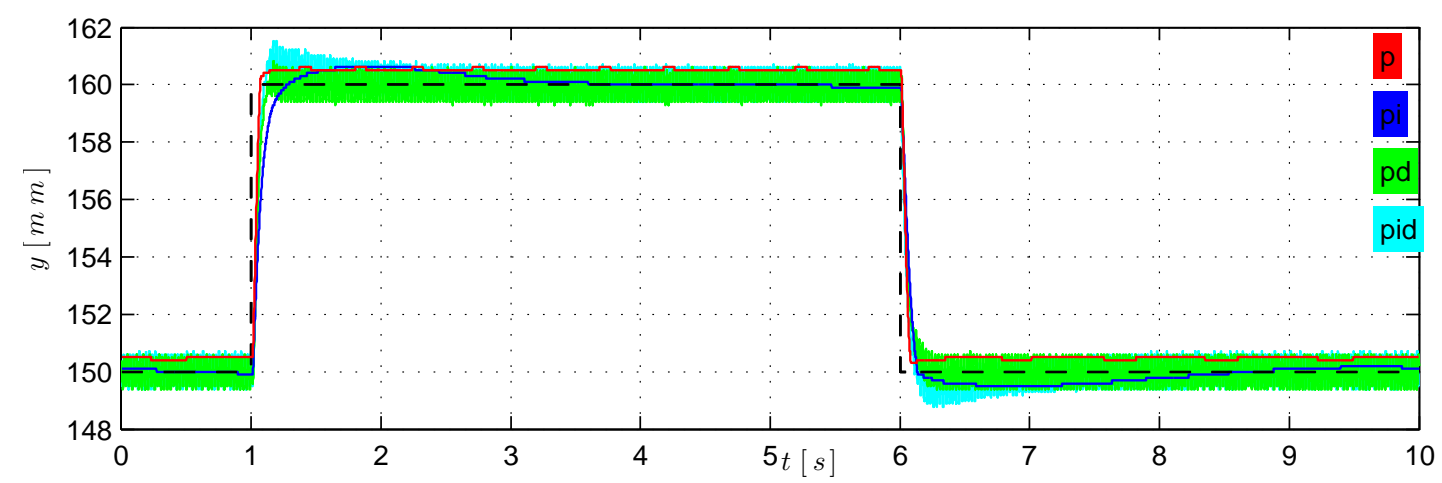

Figure 7.1: Time response of the SHA with classic controller for position control.

These curves show some revealing results:

- P controller does not provide zero steady estate error. For this purpose, a PI controller must be implemented instead.

- PD and PID controller provides a noisy time response, which are unacceptable for position tracking purposes.

From (7.2) it is seen that the system has a pole at the origin, therefore it is expected that a simple $\mathrm{P}$ controller would be enough in order to provide zero steady state error. However, it can be appreciated in the 7.1 (red curve), that this is not the case in the real system.

This can be explained by considering once again the complete model, and closing the loop as presented in Fig. 7.2.

The error $E(s)$ of the system can be found by simply observation and it is given by:

$$
E=\frac{1}{1+K_{p} G_{1}} Y_{R}+\frac{G_{2}}{1+K_{p} G_{1}} F_{E}
$$

From this expression it can be observed that the $F_{E}$ affects directly to the error. Even more, let assume that the reference input is zero (i.e. $Y_{R}=0$ ), and let consider the external perturbation as an unitary step, then, the steady state error $e_{s s}$ will be 


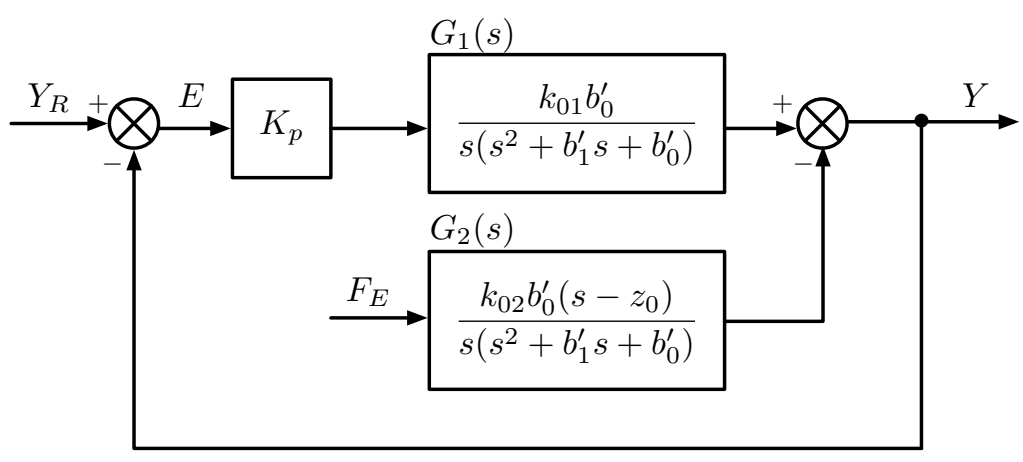

Figure 7.2: Steady state analysis

given by:

$$
\begin{aligned}
e_{s s} & =\lim _{t \rightarrow \infty} e(t), \\
& =\left.\lim _{s \rightarrow 0} s E(s)\right|_{Y_{R}=0}, \\
& =\lim _{s \rightarrow 0} s \frac{k_{02} b_{0}^{\prime}\left(s-z_{0}\right)}{s^{3}+b_{1}^{\prime} s^{2}+b_{0}^{\prime} s+K_{p} k_{01} b_{0}^{\prime}} \frac{1}{s}, \\
& =-\frac{k_{02} z_{0}}{K_{p} k_{01}},
\end{aligned}
$$

where $E(s)=\mathcal{L}\{e(t)\}$, and $K_{p}$ is the gain of the proportional controller. Thus, despite the fact that the model posses a pole at the origin, a proportional controller does not provide zero steady state error, unless $K_{p}$ is very high which is prohibitive for systems with complex conjugate pole.

Let now, consider a PI controller of the canonical form $K(s)=K_{p}+K_{i} / s$, and obtain the steady state error as follows:

$$
\begin{aligned}
e_{s s} & =\lim _{t \rightarrow \infty} e(t), \\
& =\left.\lim _{s \rightarrow 0} s E(s)\right|_{Y_{R}=0}, \\
& =\lim _{s \rightarrow 0} s \frac{k_{02} b_{0}^{\prime} s\left(s-z_{0}\right)}{s^{4}+b_{1}^{\prime} s^{3}+b_{0}^{\prime} s^{2}+K_{p} k_{01} b_{0}^{\prime} s+K_{i} k_{1} b_{0}^{\prime}} \frac{1}{s}, \\
& =0 .
\end{aligned}
$$

This analytical result can also be observed in Fig. 7.1, where it is appreciated that the time response reaches the reference value approximately in $t=4[s]$ (blue curve). However, the use of a PI controller to a first type system is controversial, since it 


\section{CONTROL STRATEGIES SHA}

will add another pole to the origin in the complex plane, thus reducing drastically the phase margin causing stability problems. Therefore the integrator time constant must be carefully selected. Despite this fact, the use of PI controller is implemented in order to increase the open loop gain at low frequencies and to decrease the steady state error (as it was demonstrated above) (72).

The stability of the system with a PI controller is analyzed in the next section, where it is proved that the SHA is robust if a suitable PI controller is implemented.

The noisy time response of the system with PD and PID controller can be analyzed by considering the well-possedness of the system. This concept is the basis of an even further powerful concept denominated the internal stability, which guarantees that all the signals of the system are bounded provided that the injected signals at any location is bounded.

Therefore, let consider the system rearranged as the standard feedback system presented in Fig. 7.3, where $K$ is the controller, and $G_{1}$ and $G_{2}$ are the transfer functions of the linearized model, $r$ is the command or reference signal, $f_{e}$ is the external force perturbation, $n$ is the sensor noise, $d_{i}$ is the disturbance at the plant input, and $d$ is the plant output disturbance.

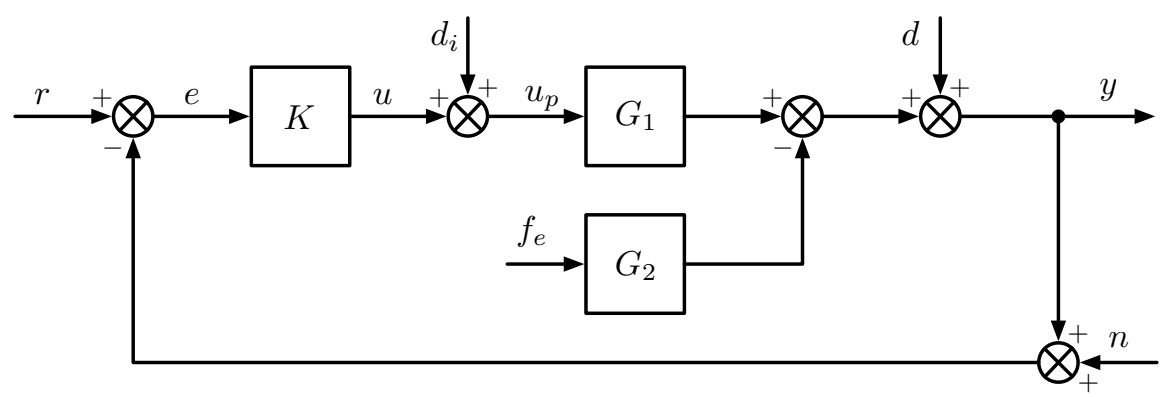

Figure 7.3: Schematic diagram for a generic internal stability analysis.

According to the Definition 5.1 related to the performance specification and limitations of feedback systems in (157):

"a feedback system is said to be well-posed if all closed-loop transfer matrices are welldefined and proper".

Therefore, after a few algebraic manipulation it can be found the following expres- 
sion for the closed loop:

$$
u=\frac{K}{1+K G_{1}}(r-n-d)-\frac{K G_{1}}{1+K G_{1}} d_{i}+\frac{K G_{2}}{1+K G_{1}} f_{e}
$$

Considering the classic P, PI, PD, PID controllers, all the transfer matrix in (7.6) are obtained. In Table 7.1 it is presented the result for $K /\left(1+K G_{1}\right)$ for all the controller. A complete Table with all the transfer matrices can be found in B.

Table 7.1: Controllers and well-posedness of the feedback system. (see B for the complete Table).

\begin{tabular}{cc} 
Controller K(s) & $\frac{K}{1+K G_{1}}$ \\
\hline P & $\frac{K_{p}\left(s^{3}+b_{1} s^{2}+b_{0} s\right)}{s^{3}+b_{1} s^{2}+b_{0} s+K_{p} k_{1} b_{0}}$ \\
PI & $\frac{\left(K_{p} s+K_{i}\right)\left(s^{3}+b_{1} s^{2}+b_{0} s\right)}{s^{4}+b_{1} s^{3}+b_{0} s^{2}+K_{p} k_{1} b_{0} s+K_{i} k_{1} b_{0}}$ \\
PD & $\frac{\left(K_{p}+K_{d} s\right)\left(s^{3}+b_{1} s^{2}+b_{0} s\right)}{s^{3}+b_{1} s^{2}+\left(b_{0}+K_{d} k_{1} b_{0}\right) s+K_{p} k_{1} b_{0}}$ \\
PID & $\frac{\left(K_{d} s^{2}+K_{p} s+K_{i}\right)\left(s^{3}+b_{1} s^{2}+b_{0} s\right)}{s^{4}+b_{1} s^{3}+\left(b_{0}+K_{d} k_{1} b_{0}\right) s^{2}+K_{p} k_{1} b_{0} s+k_{i} k_{1} b_{0}}$
\end{tabular}

As it can be observed in Table 7.1, PD and PID controllers do not provide a wellposed feedback system, since $K /\left(1+K G_{1}\right)$ is not proper, i.e. the order of the polynomial of the numerator is greater than the order of the polynomial of the denominator, and it is necessary that all the transfer matrices of the closed loop be proper. Therefore, the PD and PID controllers are prone to noisy outputs as it was found experimentally and shown in Fig. 7.1 (green and cyan curve respectively ).

\subsection{PI Controller with Robust Stability}

As it was discussed in the previous section, the main concern of implementing a PI controller with the third order system with an integrator, is whether this controller can provide stability to the system or not. 


\section{CONTROL STRATEGIES SHA}

In the previous chapter it was presented that the non linear model of the SHA, was linearized and identified for 33 different defined set points (11 piston's position and 3 external load). Thus, the linearized model presented in 4.4 should be reformulated considering the variability and dependency of its parameters to the set-point, as follows:

$$
Y(s)=\frac{\left.K_{1}(y, m) B_{0}(y, m)\right)}{s\left(s^{2}+B_{1}(y, m) s+B_{0}(y, m)\right)} X_{v}(s)+\frac{\left.K_{2}(y, m) B_{0}(y, m)\right)\left(s-Z_{0}(y, m)\right)}{s\left(s^{2}+B_{1}(y, m) s+B_{0}(y, m)\right)} F_{e}(s) .
$$

This variability on the parameters remarks the parametric uncertainty of the model.

On the other hand, as it was mentioned in 4.4.1, $F_{e}$ corresponds to external force perturbations (mainly due to gravity aspects and other external forces). Therefore for the design and analysis of the controller, it will only be considered $G_{1}(s)=Y(s) / X_{v}(s)$ as the plant, and the transfer matrix $G_{2}(s)=Y(s) / F_{e}(s)$ related with the external perturbation will be incorporated only for the analysis of robustness of the system.

Thus, without loosing generality, (7.7) can be thought as

1. A system whose parameters move from a nominal values through a bounded set:

$$
Y(s)=\frac{k_{10}\left(1+\delta_{k}\right)}{s\left(s^{2}+b_{10}\left(1+\delta_{1}\right) s+b_{00}\left(1+\delta_{0}\right)\right)} X_{v}(s),
$$

where $\delta_{k} \leq\left|\delta_{k \max }\right|, \delta_{1} \leq\left|\delta_{1 \max }\right|, \delta_{0} \leq\left|\delta_{0 \max }\right|$, and $k_{10}, b_{10}$ and $b_{00}$ correspond to the parameters of the nominal system. This formulation, which leads to a structured uncertainties definition, presents a more elegant definition of the problem, since the uncertainties can be associated to individual aspects of the physics phenomenom of the system.

2. A system bounded by some weighting functions that characterizes the spatial and frequency structure of the uncertainty $\Delta(s)$. One of the many canonical formulations is the well known multiplicative uncertainty definition as follows:

$$
\Pi(s)=\left(1+W_{1}(s) \Delta(s) W_{2}(s)\right) P_{0},
$$

where $P_{0}(s)$ is the nominal plant, $W_{1}(s)$ and $W_{2}(s)$ are stable transfer matrices, and $\|\Delta\| \leq 1$. This particular representation of the set of plants, confines $\Pi(s)$ to a normalized neighborhood of the nominal model $P_{0}(s)$. This type of formulation, known as unstructured uncertainties definition, presents the problem as 
a bounding problem, which does not require much knowledge of the system and may lead to conservative solutions. However, this formulation is much simpler.

It is important to recall that both formulations require of the definition of a nominal plant.

\subsubsection{Nominal Plant and Uncertainties Definitions}

In this work, the nominal plant is defined as the identified plant that best fit to all the input and output data collected during the experimental identification. Basically, all the plants are simulated and compared with all the input-output data. The plant that maximizes (7.10), is selected as the nominal plant.

$$
\max _{\forall G_{1}(s)} \sum_{i=1}^{33}\left\|F_{i}\right\|,
$$

where:

$$
F_{i}=\frac{1-\left\|y_{s i}-y_{i}\right\|}{\left\|y_{i}-\bar{y}_{i}\right\|}
$$

where, $y_{s_{i}}$ is the simulated output of $G_{1}(s)$ with the ith input register, $y_{i}$ is the $i t h$ output resgister, and $\bar{y}_{i}$ is its mean.

According to this criteria, the nominal plant corresponds to the following identified plant:

$$
\begin{aligned}
& P_{0}(s)=P_{6 k g, 120 m m}(s), \\
& P_{0}(s)=\frac{1.72210^{6}}{s^{3}+90.31 s^{2}+7692 s} .
\end{aligned}
$$

\subsubsection{Unstructured uncertainty definition}

As it is introduced in (7.9), the system is bounded on the frequency domain by weightings transfer function. Therefore, in order to obtain the unstructured uncertainty bound for this system, first it must be found the discrepancy between the set of plants and the nominal plant, as follows:

$$
W_{1}(s) \Delta(s) W_{2}=\frac{\Pi(s)-P_{0}(s)}{P_{0}(s)} .
$$

Therefore, considering a frequency range of $w \in\left[10^{-2}, 10^{3}\right]$, an error plot is generated and presented in Fig.7.4. From this plot (using the ginput command of Matlabß) it is 


\section{CONTROL STRATEGIES SHA}

selected a set of upper-bound frequency response (beyond the maximum errors found presented in dashed red). This set of points are fitted to a stable and minimum phase third order transfer function, which defines the bounding weighting transfer function and thus the multiplicative uncertainty.

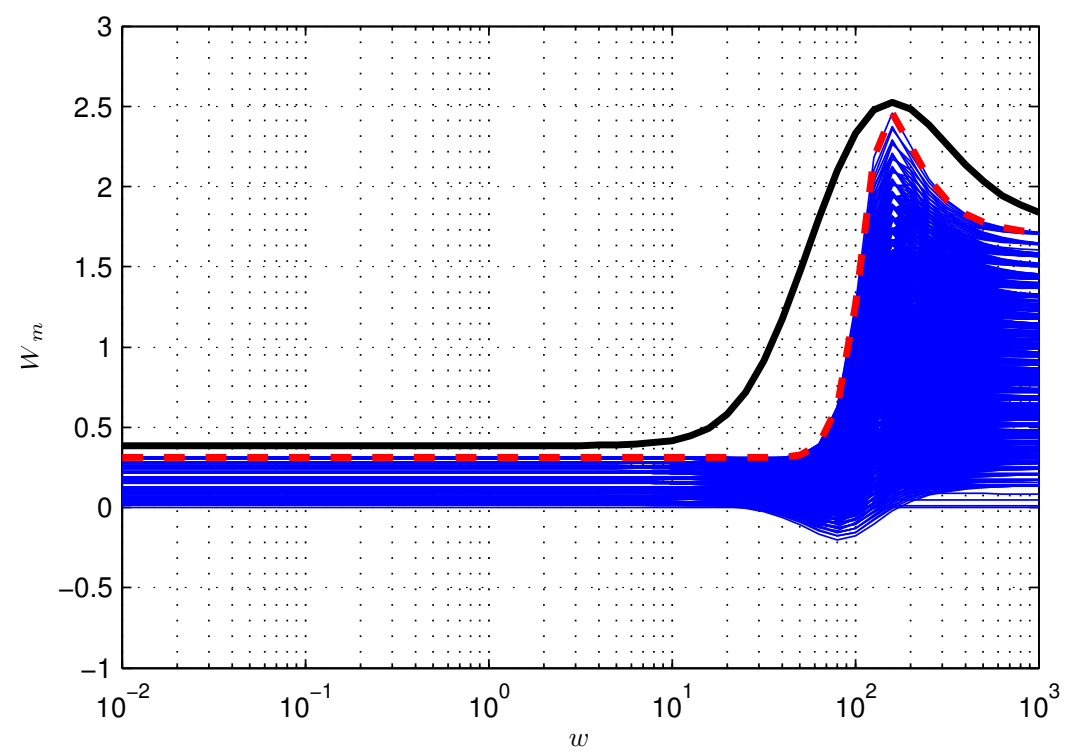

Figure 7.4: Multiplicative uncertainty definition. blue: error plot. red: maximum error. black: multiplicative uncertainty weighting transfer function.

In order to facilitate de analysis and with out loosing generality, it is considered that $W_{2}(s)=1$, therefore the weighting function is given by:

$$
W_{1}(s)=\frac{1.759 s^{3}+724.9 s^{2}+2.15910^{6} s+2.40610^{5}}{s^{3}+313.9 s^{2}+2.6510^{4} s+6.27510^{5}}
$$

\subsubsection{Controller}

The first controller implemented is a classical PI, with a anti-windup (see Fig. 7.5). This controller despite the fact that is very simple, provides certain robustness, as it is proved in the following sections.

The proportional and integral gain of the controller is tuned using the ZieglerNichols technique with the nominal plant, which provides the following values: $K_{p}=$ 0.085 and $K_{i}=0.46$. 


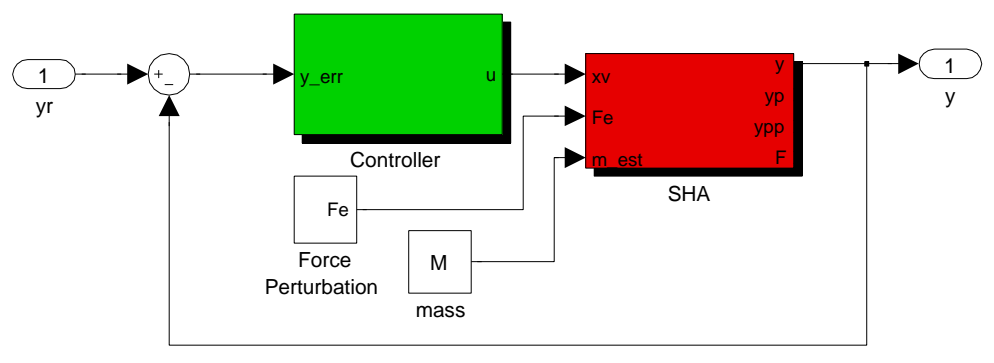

(a)

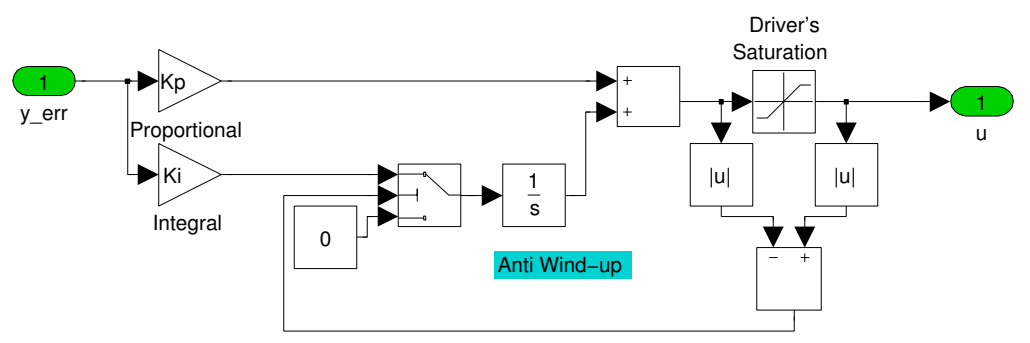

(b)

Figure 7.5: PI Controller with anti-windup.

\subsubsection{Robustness Analysis}

In the following paragraphs, the robustness of the system with the classical PI controller, will be proved.

\subsubsection{Internal Stability (Nominal Stability)}

Internal stability guarantees that all signals in a system are bounded provided that the injected signal at any location are bounded.

Therefore, lets arranged the linear feedback system under analysis presented in Fig.7.6(a), as the canonical form presented in Fig.7.6(b).

Definition 1 The system of Fig.7.6(b) is said to be internally stable if the transfer 


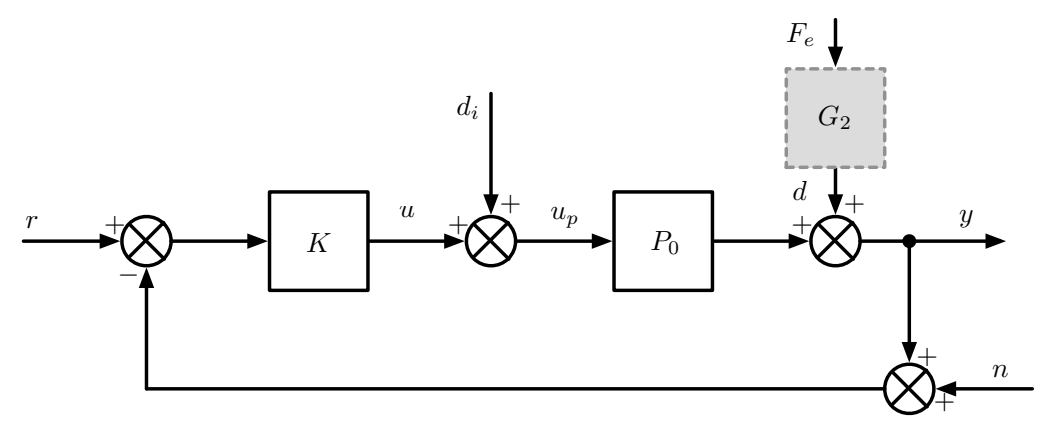

(a)

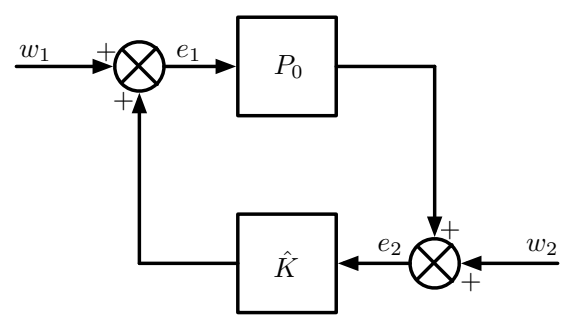

(b)

Figure 7.6: Schematic diagram for Internal Stability Analysis.

matrix:

$$
\begin{aligned}
{\left[\begin{array}{cc}
I & -\hat{K} \\
-P & I
\end{array}\right]^{-1} } & =\left[\begin{array}{cc}
(I-\hat{K} P)^{-1} & \hat{K}(I-P \hat{K})^{-1} \\
P(I-\hat{K} P)^{-1} & (I-P \hat{K})^{-1}
\end{array}\right] \\
& =\left[\begin{array}{cc}
I+\hat{K}(I-P \hat{K})^{-1} P & \hat{K}(I-P \hat{K})^{-1} \\
(I-P \hat{K})^{-1} P & (I-P \hat{K})^{-1}
\end{array}\right]
\end{aligned}
$$

from $\left(w_{1}, w_{2}\right)$ to $\left(e_{1}, e_{2}\right)$ to $\mathcal{R H}_{\infty}(157)$.

Therefore, the four transfer matrices presented in the right side of (7.16) must belong to $\mathcal{R H} \mathcal{H}_{\infty}$, i.e. that all the poles of the transfer matrices are in $\mathbb{C}^{-}$. These 
transfer functions are stated in the following expressions:

$$
\begin{aligned}
(I-\hat{K} P)^{-1} & =\frac{s^{2}\left(s^{2}+b_{1} s+b_{0}\right)}{s^{4}+b_{1} s^{3}+b_{0} s^{2}+K_{p} k_{1} b_{0} s+K_{i} k_{1} b_{0}} \\
P(I-\hat{K} P)^{-1} & =\frac{s k_{1} b_{0}}{s^{4}+b_{1} s^{3}+b_{0} s^{2}+K_{p} k_{1} b_{0} s+K_{i} k_{1} b_{0}} \\
\hat{K}(I-P \hat{K})^{-1} & =\frac{s k_{1} b_{0}}{s^{4}+b_{1} s^{3}+b_{0} s^{2}+K_{p} k_{1} b_{0} s+K_{i} k_{1} b_{0}} \\
(I-P \hat{K})^{-1} & =\frac{s^{2}\left(s^{2}+b_{1} s+b_{0}\right)}{s^{4}+b_{1} s^{3}+b_{0} s^{2}+K_{p} k_{1} b_{0} s+K_{i} k_{1} b_{0}}
\end{aligned}
$$

As it can be observed the denominator in all transfer function are the same, and whose poles are:

$$
\begin{aligned}
p_{1,2} & =-33.3028 \pm 70.4713 i \\
p_{3} & =-20.0222 \\
p_{4} & =-3.3723
\end{aligned}
$$

Since all the poles belong to $\mathbb{C}^{-}$, the system presented in Fig.7.6(b) is internally stable.

\subsubsection{Robust Stability}

In Fig. 7.7 it is presented a reduced Nyquist diagram of $\Pi(s)$. It can be observed how the uncertainty dilates the diagram of the nominal plant.

Therefore, in order to provide robust stability to a system, the controller $K(s)$ must internally stabilizes every plant belonging to $\Pi(s)=P_{0}(s)\left(1+W_{1}(s) \Delta(s)\right)$.

The basis for the robust stability criteria is derived form the small gain theorem which states:

Theorem 1 (Small Gain Theorem) Suppose $M \in \mathcal{R H}_{\infty}$ and let $\gamma>0$. Then the interconnected system presented in Fig.7.8 is well posed and internally stable for all $\Delta(s) \in \mathcal{R H}_{\infty}$ with

1. $\|\Delta(s)\|_{\infty} \leq 1 / \gamma$ if and only if $\|M(s)\|_{\infty}<\gamma$.

2. $\|\Delta(s)\|_{\infty}<1 / \gamma$ if and only if $\|M(s)\|_{\infty} \leq \gamma$.

Hence, the linear feedback system with the unstructured multiplicative uncertainty presented in Fig. 7.9(a), can be easily arranged into the canonical $M-\Delta$ loop (see Fig. $7.9(\mathrm{~b}))$. 


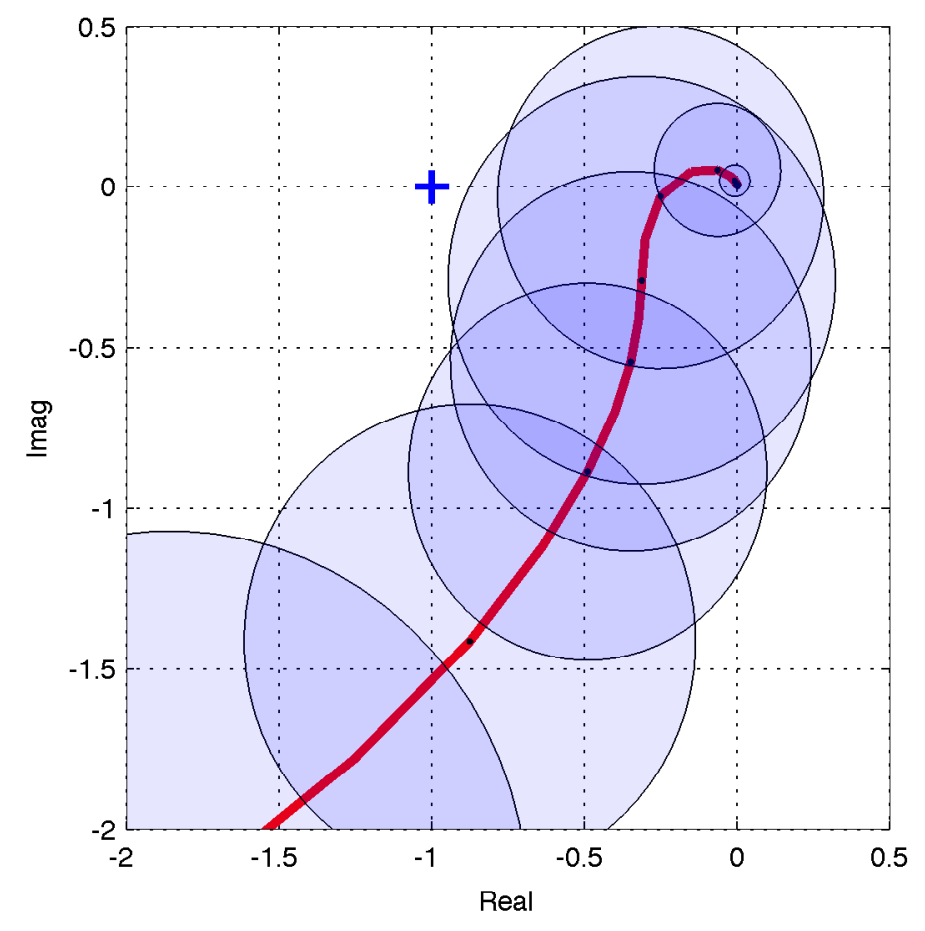

Figure 7.7: Nyquist diagram for $\Pi(s)$. Nominal plant in red. Uncertainty in blue disks.

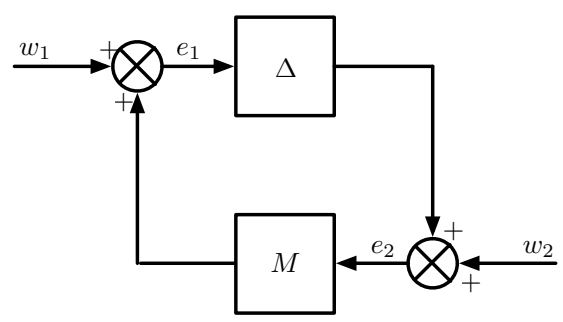

Figure 7.8: $M-\Delta$ loop for stability analysis.

Based in this scheme and applying Theorem $\mathbf{8 . 5}$ from (157) derived from the small gain theorem, that states:

Theorem 2 Let $\Pi:\left\{\left(I+W_{1} \Delta\right) P_{0}: \Delta \in \mathcal{R H}_{\infty}\right\}$ and let $K$ be a stabilizing controller for the nominal plant $P_{0}$. Then the closed loop system is well-posed and internally sable for all $\Delta \in \mathcal{R H}_{\infty}$ with $\|\Delta\|_{\infty}<1$ if and only if $\left\|W_{1} T\right\|_{\infty} \leq 1$.

Since by definition $\Delta \in \mathcal{R} \mathcal{H}_{\infty}$ and $\|\Delta\|_{\infty}<1$, and furthermore $K$ stabilizes the 


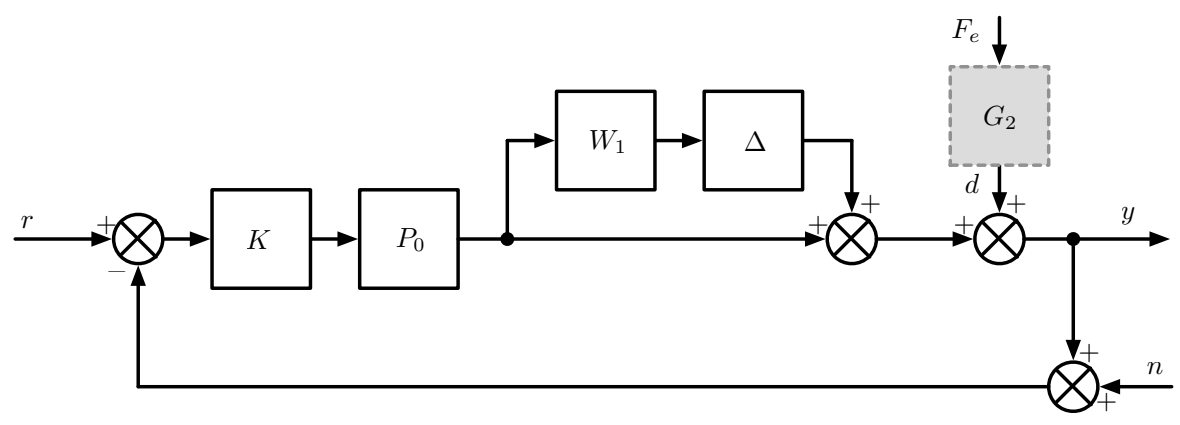

(a)

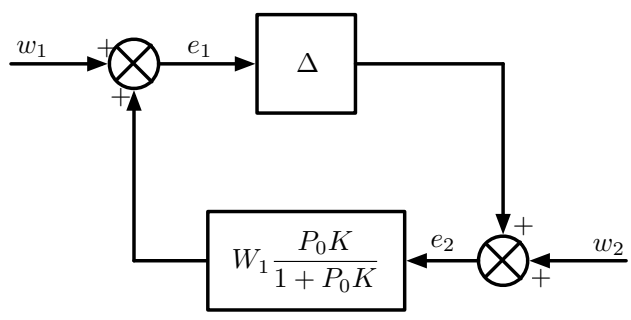

(b)

Figure 7.9: Multiplicative perturbed system. 7.9(a): linear feedback system. 7.9(b): $M-\Delta$ loop arrangement.

nominal plant $P_{0}$, and on the other hand:

$$
\begin{aligned}
\left\|W_{1}(j w) T(j w)\right\|_{\infty} & =\left\|W_{1}(j w) \frac{P_{0}(j w) K(j w)}{1+P_{0}(j w) K(j w)}\right\|_{\infty} \\
& =0.8523 \leq 1,
\end{aligned}
$$

then, the system is internally stable.

\subsubsection{Robust Performance}

Lets consider the general perturbed feedback system presented in Fig.7.9(a). As it was mentioned previously $G_{2}$ is associated to the action of external forces, and it could be considered as a weighting matrix function for the external perturbation.

Thus, the performance robustness of the system is defined as how does the external perturbation affects to the output.

A simple criteria could be to keep the worst-case energy of the output as small as possible over a unit energy input $\tilde{d}$. 


\section{CONTROL STRATEGIES SHA}

Therefore, the robust performance criterion can be described as requiring that the closed-loop system be robustly stable and that transfer matrix $\left\|T_{y \tilde{d}}\right\|_{\infty} \leq 1$.

Lets first analyze the origins of $G_{2}$. As it was presented in 4.4.1, the parameters of this transfer function are variable according to the current set-point. Therefore, in reality $G_{2}$ is a set of transfer function, which for simplicity it will be defined as the bounded set: $\Omega:\left\{\left(1+W_{d m} \Delta_{d}\right) W_{0}: W_{d m}, W_{0}, \Delta_{d} \in \mathcal{R H}_{\infty},\left\|\Delta_{d}\right\|_{\infty} \leq 1\right\}$. This kind of multiplicative unstructured definition for the set of weighting functions, provides a better approach to the formulation of the problem. Where $W_{0}$ is a the nominal weighting function and $W_{d m}$ is the multiplicative bounding weight.

The nominal weighting function, will be defined with the parameters of the identified plant used as $P_{0}$, i.e.:

$$
W_{0}(s)=\frac{0.1372 s+11.93}{s^{3}+90.31 s^{2}+7692 s}
$$

For the definition of the multiplicative weighting function $W_{d m}$, the same procedure used for the definition of $W_{1}$ is used. The resulting, weighting transfer function is:

$$
W_{d m}(s)=\frac{2.951 s^{3}+781.4 s^{2}+2.6610^{4} s+5342}{s^{3}+240.6 s^{2}+1.31910^{4} s+2695} .
$$

Considering these elements, by simple observation of Fig. 7.9(a) it can be found that:

$$
\begin{aligned}
T_{y \tilde{d}} & =(1+K \Pi)^{-1} \Omega \\
& =\left[1+\left(1+W_{1} \Delta\right)\right]^{-1}\left(1+W_{d m} \Delta_{d}\right) W_{0} .
\end{aligned}
$$

The robust stability given by $\left\|W_{1} T_{o}\right\|_{\infty} \leq 1$ was proved in the previous paragraph, and since:

$$
\left\|T_{y \tilde{d}}\right\|_{\infty}=2.143410^{-4} \leq 1
$$

the system presents robust performance for bounded external forces.

\subsection{Adaptive Hybrid Force-Position Controller}

The robust PI controller presented previously attempts to keep consistent performance under possible external perturbation and uncertainties. However, in principle, adaptive controller is superior with robust controller when dealing with uncertainties in constant 
or slowly varying parameter. Indeed, adaptive controller may improve its performance as adaptation goes on.

Therefore, in this section, it is introduced a controller whose parameters adapts to the current set point and it also includes a force feedback loop to provide compensations to external force perturbations, turning the controller into a hybrid force-position controller.

Basically, the controller is much like a Gain Scheduling controller, and the adaptation law is based on the estimation of the current set-point (i.e. position and external load), selecting the set of parameters from a surface defined by the spline interpolation of a table of parameters defined by each identified plant. The schematic diagram of the controller is presented in Fig. 7.10.

As it can be observed, the diagram presents a Friction Observer block, a Load Pressure Estimation, and Inverse Dynamic calculation in the forward loop and a estimation of the external load block.

The adaptation of the controller is governed by the estimation of the external load and the current position.

All the blocks are detailed in the following paragraphs.

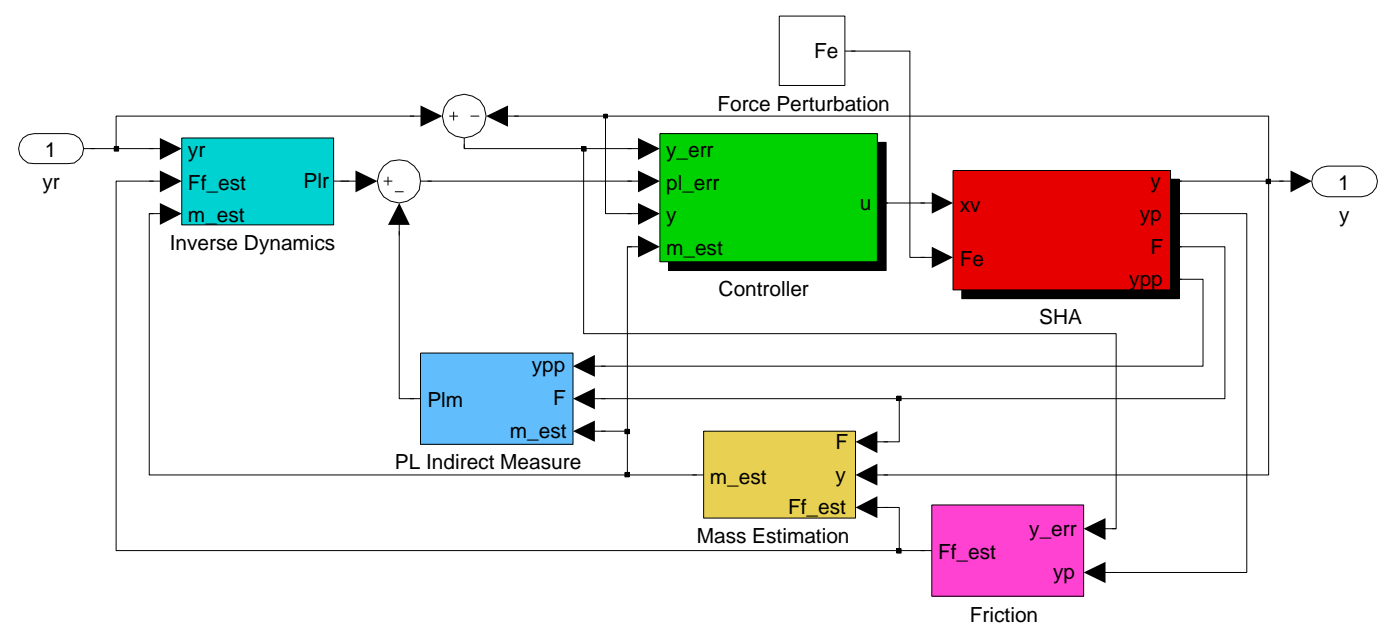

Figure 7.10: Simulink model for the Adaptative Hybrid Controller, Ff_estimated $=\hat{F}_{f}$, Fe_estimated $=\hat{F}_{e}, m_{-}$estimated $=\hat{m}, \quad$ PL_err $=\tilde{P}_{L}, \quad$ y_err $=\tilde{y}, \quad$ PL_est $=\hat{P}_{L}$, ypp $=\ddot{y}, \quad$ yp $=\dot{y}$, sigma $=\sigma$. 


\section{CONTROL STRATEGIES SHA}

\subsubsection{Force-Position Controller Description}

The control law of the system is derived from the previous position tracking PI controller, to whom a force loop is added. The latter one is intended to compensate the external force actions such as a friction and gravity.

The structure of the controller is given by (see Fig. 7.11):

$$
u=K_{p l} \tilde{P}_{L}+K_{p} \tilde{y}+K_{i} \int \tilde{y} d t
$$

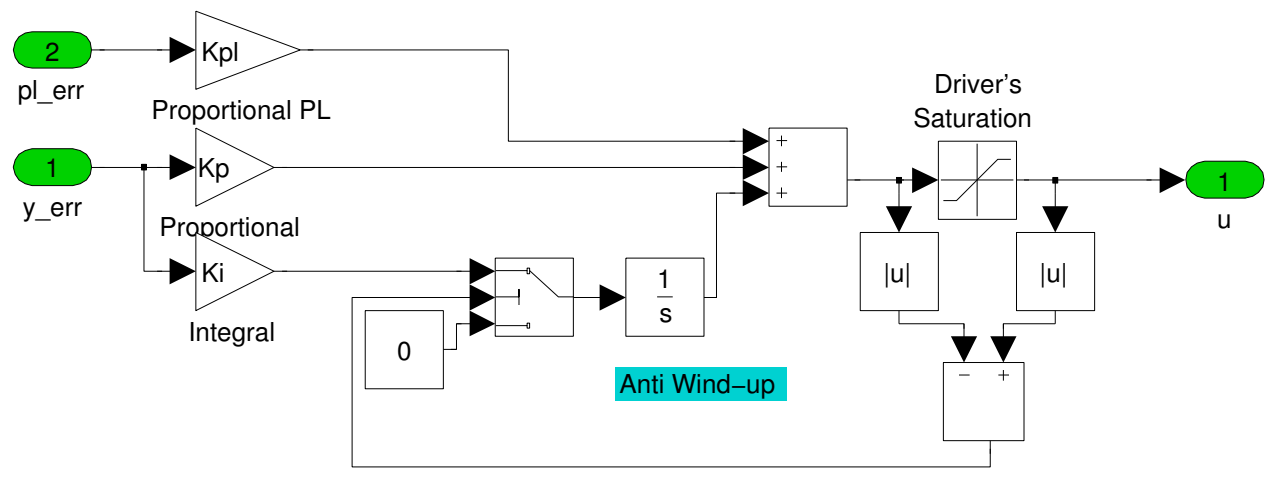

Figure 7.11: Local force-position controller.

\subsubsection{Position PI Controllers tuning}

A PI controller is tuned for each identified plant by simulation in Simulink@, implementing the Ziegler-Nychols technique, and further validated in the Test-bed (126).

A typical time response for the PI controller is presented in Fig. 7.12(a). As it can be observed, the responses obtained from simulation and experimentation are quite similar, which validates the identification process previously performed. On the other hand, it can be also seen that the time response presents an overshoot bigger than $70 \%$ along with a decaying oscillations dynamics, which are not preferable for positioning purposes. Therefore the controller must be adjusted manually in order to improve the time response by reducing its overshoot and further eliminate the oscillations. This goal can be achieved by reducing the integral term of the PI controller, as it is shown in Fig. 7.12(b). 


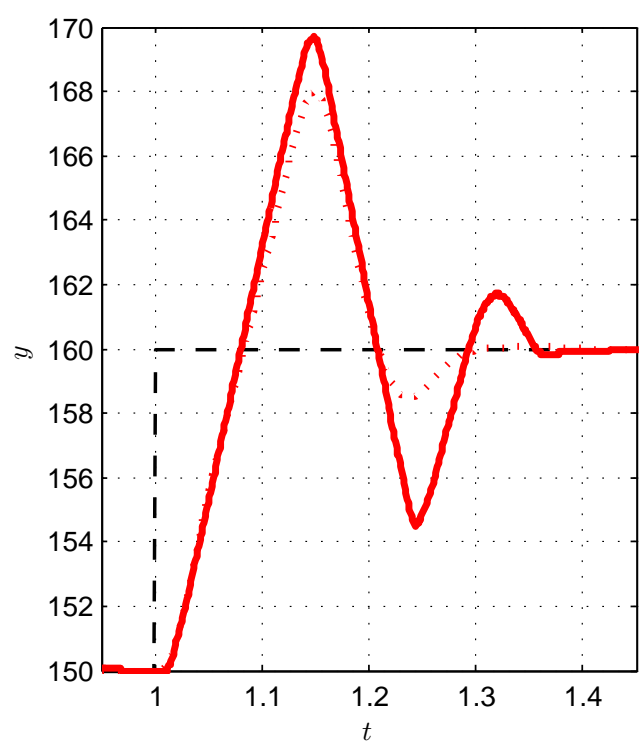

(a)

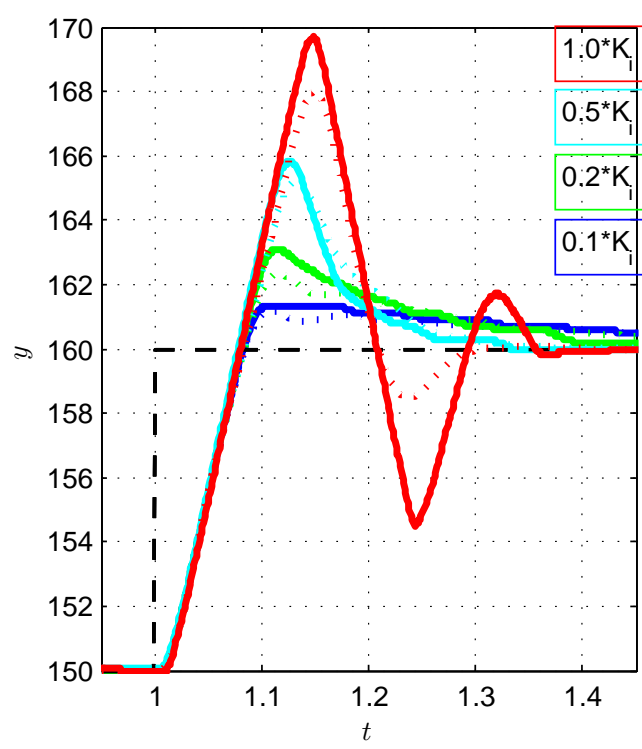

(b)

Figure 7.12: PI controller time response. Experimental time response in full line, and simulations in dot line.

Taking a closer look at the time responses for the different integral terms of the PI controller (see Fig. 7.12(b)), it can be seen that the response for $K_{i}^{\prime}=0.2 K_{i}$, presents an overshoot approximated of $25 \%$ and an establishing time of $500 \mathrm{~ms}$. This result is almost invariant for all the controllers.

Thereby, all the integral terms of the PI controllers obtained with the ZN technique, are modified with this later criteria, resulting in the PI controllers presented in Table 7.2 .

\subsubsection{Controller Implementation}

The controller must be adapted according to the current set point. This adaptation or switching between controllers also known as gain scheduling can be performed by several strategies (e.g. fuzzy logic [refs], markov criteria [refs], etc).

Gain scheduling is conceptually simple, and, indeed, practically successful for a number of applications. The main problem with gain scheduling is that has only limited theoretical guarantees of stability in nonlinear operation, but uses some loose practical 
Table 7.2: PI Controllers

\begin{tabular}{c|cc|cc|cc}
\hline & \multicolumn{2}{|c|}{$0 \mathrm{~kg}$} & \multicolumn{2}{c|}{$3 \mathrm{~kg}$} & \multicolumn{2}{c}{$6 \mathrm{~kg}$} \\
$P_{0}$ & $K_{p}$ & $K_{i}$ & $K_{p}$ & $K_{i}$ & $K_{p}$ & $K_{i}$ \\
\hline \hline 10 & 0.128 & 2.747 & 0.097 & 2.428 & 0.1 & 2.493 \\
30 & 0.122 & 2.633 & 0.097 & 2.535 & 0.098 & 2.499 \\
60 & 0.114 & 2.46 & 0.096 & 2.606 & 0.094 & 2.475 \\
90 & 0.107 & 2.305 & 0.092 & 2.607 & 0.089 & 2.427 \\
120 & 0.102 & 2.189 & 0.088 & 2.573 & 0.085 & 2.378 \\
150 & 0.098 & 2.13 & 0.085 & 2.544 & 0.082 & 2.347 \\
180 & 0.098 & 2.135 & 0.083 & 2.539 & 0.08 & 2.343 \\
210 & 0.101 & 2.203 & 0.083 & 2.565 & 0.079 & 2.372 \\
240 & 0.106 & 2.324 & 0.084 & 2.618 & 0.079 & 2.434 \\
270 & 0.112 & 2.48 & 0.086 & 2.684 & 0.081 & 2.522 \\
290 & 0.117 & 2.593 & 0.087 & 2.728 & 0.082 & 2.592 \\
\hline
\end{tabular}

guidelines such as the scheduling variables should change slowly and the scheduling variables should capture the plant's nonlinearities.

Therefore, in this work, the selection of an appropriate set of parameters for the controller is found by cubic interpolation between the individual controllers, providing continuity at the controller and smooth variations.

Thus, the control law is defined as:

$$
u=\mathcal{C}_{p l}\left(y, m_{L}\right) \tilde{P}_{L}+\mathcal{C}_{p}\left(y, m_{L}\right) \tilde{y}+\mathcal{C}_{i}\left(y, m_{L}\right) \int \tilde{y} d t,
$$

where $\mathcal{C}_{p l}, \mathcal{C}_{p}$ and $\mathcal{C}_{i}$ are the surfaces defined by the table of controller for each set point.

\subsubsection{Friction Estimation}

The friction estimation is based on the friction model given by (6.1), (6.2) and (6.3),

$$
\begin{aligned}
\dot{\hat{z}} & =\dot{y}-\sigma_{0} \frac{|\dot{y}|}{g(\dot{y})} \hat{z}-k \tilde{y} \\
g(\dot{y}) & =\alpha_{0}+\alpha_{1} e^{\dot{y} / v_{s}{ }^{2}} \\
\hat{F}_{f} & =\sigma_{0} \hat{z}+\sigma_{1} \dot{\hat{z}}+\sigma_{2} \dot{y}
\end{aligned}
$$




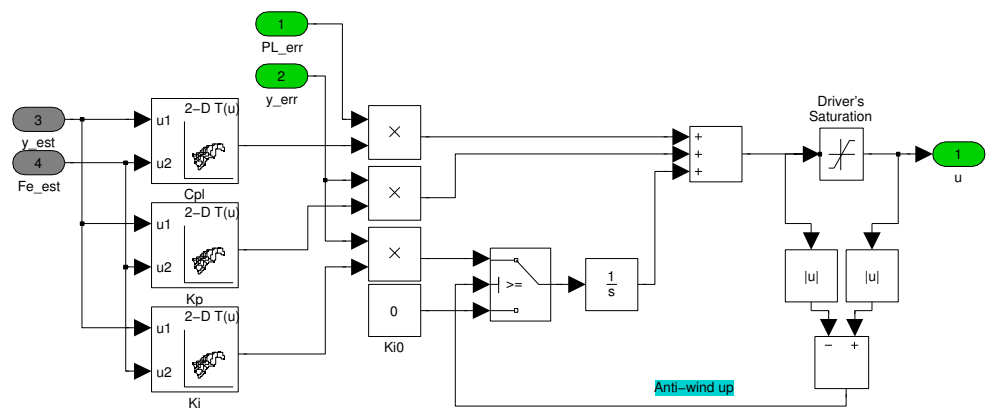

Figure 7.13: Adaptative Controller.

where $\hat{z}$ is the estimated internal state $z$ and $\hat{F}_{f}$ is the estimated friction force (89).

\subsubsection{External Load Mass Estimation}

The external load mass, along with the rod's position define the set-point of the SHA, and therefore the set of parameter of the controller.

The external force perturbations, can be thought as a dynamical variable mass load, thus under this consideration, the dynamics of this mass can be stated as:

$$
m_{L} \ddot{y}=A_{p} P_{L}-F_{f}-F_{e}
$$

where $F_{e}=m_{L} g$, and $m_{L}$ is the equivalent external load mass, and $g$ is the gravity acceleration.

From the other hand, the system posses only two measured variable, the net force acting along the piston $F$, and its position $y$. The net force, obtained from the stresscompression force sensor, measures the following relation:

$$
F_{s}=A_{p} P_{L}+F_{f}+F_{e}
$$

Equating the last two expression it can be found the following relation:

$$
m_{L} \ddot{y}+2 F_{f}+2 m_{L} g=F_{s},
$$

Now, considering that $F_{f}$ can be estimated, (7.35) can be expressed as a linear relation between the known data and the unknown parameter as follows:

$$
m_{L}(\ddot{y}+2 g)=\left(F_{s}-2 \hat{F}_{f}\right)
$$




\section{CONTROL STRATEGIES SHA}

However, the usage of this expression is not recommended for estimation, since the second derivative of $y$ appears in the linear model, and numerically differentiating $y$ is undesirable because of noise considerations (56). To eliminate $y$ in the above equation, a filter $F(s)$ is implemented at both sides of the expression, and abusing of the notation (7.36) can be formulated as follows:

$$
m_{L} F(s)\left\{\left(2 g+s^{2} y(t)\right)\right\}=F(s)\left\{F_{s}(t)-2 \hat{F}_{f}(t)\right\}
$$

From this last model, $m_{L}$ can be estimated using a least squares approach. Considering, that the estimation must be performed on-line and it is assumed that $m_{L}$ varies smoothly, a recursive least square with exponentially forgetting algorithm is implemented.

A typical set of curves during one of the experiments detailed bellow, is presented in Fig. 7.14. In these charts, is depicted the action of the mass estimation block for different external load mass.

\subsubsection{Indirect Measure of Load Pressure}

An indirect measure of the load pressure $P_{L}$ can be easily derived by adding (7.33) and (7.34), resulting into :

$$
2 A_{p} P_{L}=\hat{m}_{L} \ddot{y}+F_{s},
$$

and further, solving for $P_{L}$ as follows:

$$
P_{L}=\frac{1}{2 A_{p}}\left(\hat{m}_{L} \hat{\ddot{y}}+F_{s}\right),
$$

where $\hat{\ddot{y}}$ is obtained from a second-order low-pass filtered differentiator.

This last expression avoids using an extra estate estimator, and thus reducing the computational costs. In Fig. 7.15, it is presented a comparison between the indirect $P_{L}$ measurement and a direct measurement from the pressure sensor of the SHA.

\subsubsection{Inverse Dynamics}

The desired load pressure at the SHA $\left(P_{L_{r}}\right)$, is derived from the piston's equation of motion, considering the desired input command, resulting into the inverse dynamics of the pistons given by:

$$
P_{L r}=\frac{1}{A p}\left(\hat{m} \ddot{y}_{r}-\hat{F}_{f}-\hat{m} g\right) .
$$



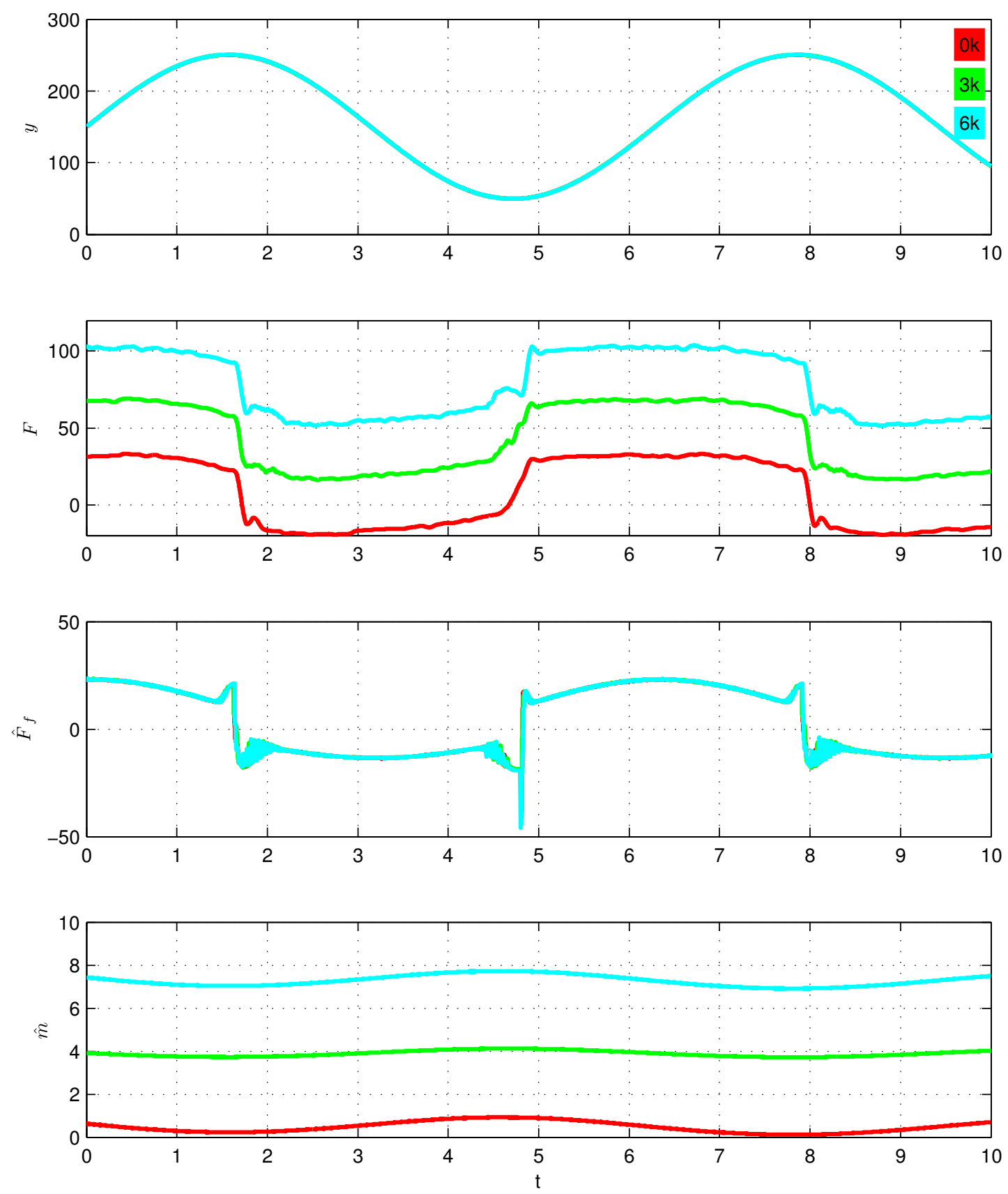

Figure 7.14: Load mass estimation. 


\section{CONTROL STRATEGIES SHA}
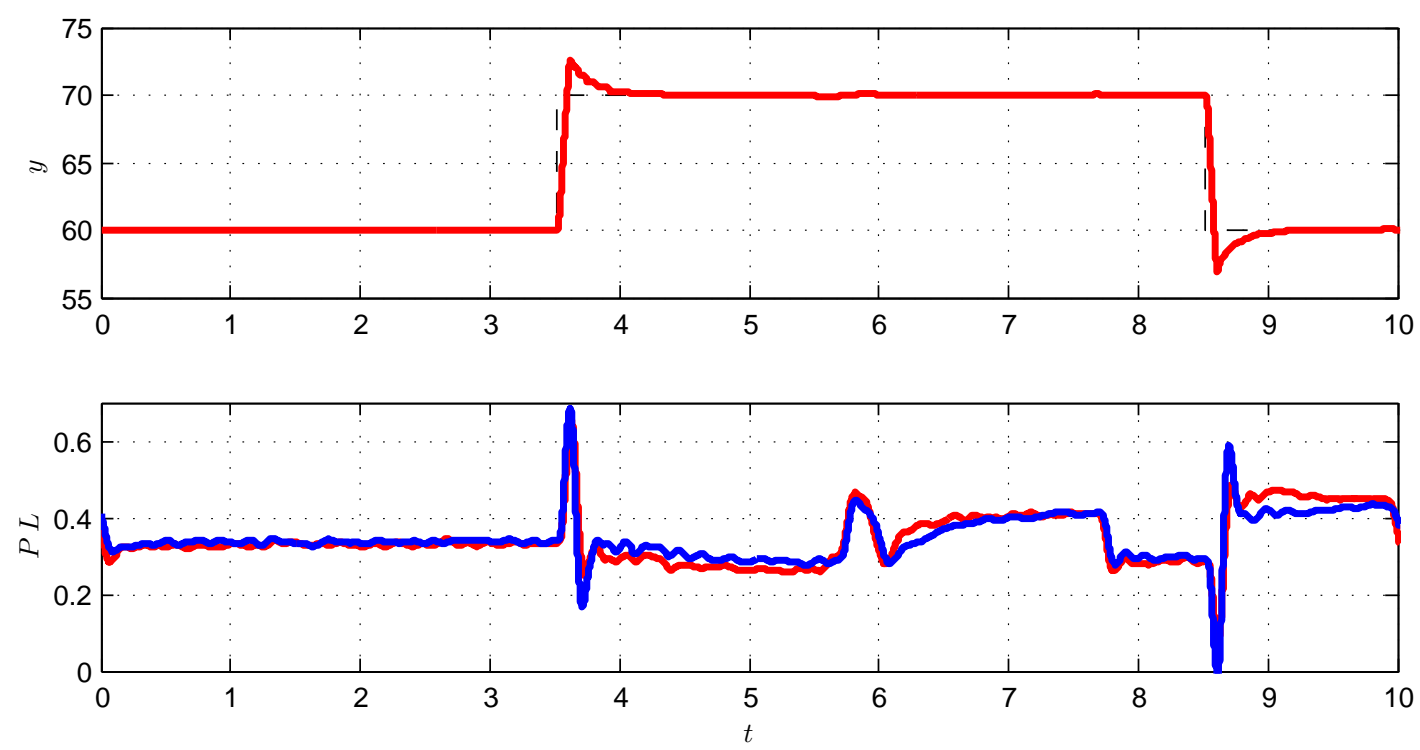

Figure 7.15: Indirect $P_{L}$ measurement.

\subsubsection{Velocity and Acceleration estimation}

It must be appreciated that some of the expression presented above, requires of the velocity and acceleration exerted by the actuator, since these variables are not directly measured they must be estimated.

The main issue regarding a digital encoder is its finite resolution. This gap between two consecutive data from the digital sensor derives in the impossibility of detecting small changes of the measured variable. Therefore, the estimation of velocity and acceleration will be mostly affected in the low-speed range.

A complete analysis of the performance from several approaches was performed and the main outcomes are summed up (120). As a consequence of this study it was find out that the Kalman Filter with adaptive correlation convariance matrices.

The estimation problem is solved recursively by implementing the following sequen- 
tial relations at each sample time ((150)):

$$
\begin{aligned}
\hat{\boldsymbol{x}}_{k}^{-} & =\boldsymbol{A} \hat{\boldsymbol{x}}_{k-1}+\boldsymbol{B} \boldsymbol{u}_{k}, \\
\boldsymbol{P}_{k}^{-} & =\boldsymbol{A} \boldsymbol{P}_{k-1} \boldsymbol{A}^{T}+\boldsymbol{Q}, \\
\boldsymbol{K}_{k} & =\frac{\boldsymbol{P}_{k}^{-} \boldsymbol{H}^{T}}{\boldsymbol{H} \boldsymbol{P}_{k}^{-} \boldsymbol{H}^{T}+\boldsymbol{R}}, \\
\hat{\boldsymbol{x}}_{k} & =\hat{\boldsymbol{x}}_{k}^{-}+\boldsymbol{K}_{k}\left(\boldsymbol{z}_{k}-\boldsymbol{H} \hat{\boldsymbol{x}}_{k}^{-}\right), \\
\boldsymbol{P}_{k} & =\left(\mathbf{1}-\boldsymbol{K}_{k} \boldsymbol{H}\right) \boldsymbol{P}_{k}^{-},
\end{aligned}
$$

For the particular case of velocity and acceleration estimation from position data, the process model can be easily derived by considering the Taylor series expansion of each state variable and assuming that the plant noise is the discrete representation of the remainder of the Taylor series ((156), (128), (136), (24)). Therefore, the process and measurement models are given by:

$$
\begin{aligned}
& {\left[\begin{array}{l}
\hat{y}_{k} \\
\hat{y}_{k} \\
\hat{y}_{k}
\end{array}\right]=\left[\begin{array}{ccc}
1 & T & T^{2} / 2 \\
0 & 1 & T \\
0 & 0 & 1
\end{array}\right]\left[\begin{array}{c}
y_{k} \\
\dot{y}_{k} \\
\ddot{y}_{k}
\end{array}\right]+\left[\begin{array}{c}
T^{3} / 6 \\
T^{2} / 2 \\
T
\end{array}\right],} \\
& y_{k+1}=\hat{y}_{k}+v_{k},
\end{aligned}
$$

and the covariance matrices are:

$$
\begin{aligned}
\boldsymbol{Q} & =q\left[\begin{array}{ccc}
T^{5} / 20 & T^{4} / 8 & T^{3} / 6 \\
T^{4} / 8 & T^{3} / 6 & T^{2} / 2 \\
T^{3} / 6 & T^{2} / 2 & T
\end{array}\right], \\
R & =\frac{e_{r}^{2} / 4+2 r}{3}
\end{aligned}
$$

where $r=E\left[\epsilon_{i}\right]$ and $\epsilon_{i}$ is the error associated to the measurement. A comprehensive study of the stochastic components of the model is presented in $((24))$. In order to enhance the performance of the KF many authors propose to modify the noise covariance matrices considering the last estimation ((136), (156)), or rescaling the gain of the filter $((51))$ turning it into an adaptive filter. In this work, it is proposed the following adaptive law:

$$
R a_{k}=\frac{10 R}{1+\hat{\dot{y}}_{k-1}^{2}} .
$$

This adaptation law allows to preserve the performance of the estimation along the full range of work providing a better result 


\section{CONTROL STRATEGIES SHA}

\subsection{Experiments}

The following section presents several a series of experiments that allows to compare the performance of the controllers proposed.

\subsubsection{Experiment \# 1: Step response}

\section{- Objective}

Analyze the characteristics of time response of the system in closed loop for different set points.

\section{- Description}

The SHA is submitted to step inputs of $10[\mathrm{~mm}]$ of amplitude in closed loop implementing the PI controller and the Adaptive controller, driving external loads of $m_{\text {ext }}:=\{0,3,6\} \mathrm{Kg}$, and working position, $y_{0}:=\{50,150,250\} \mathrm{mm}$.

\section{- Data Processing}

In order to compare the results from the different set point, all the curves obtained during experimentation are synchronized with the step input and the and it is eliminated the bias signal.

\section{- Results}

The time response of the systems for the system under PI controller is presented in Fig.7.16(a). As it can be observed, the time response are very similar even thought the system is working in different set-points, presenting an overshoot of $\approx 25 \%$ with no oscillation. The response of the system stabilizes near the $500 \mathrm{~ms}$. In Fig. 7.16(b) is presented the time response of the system in closed loop using the adaptive controller. These curves, depict a variation on the characteristics of the time response for the different set-point. The overshooting and the settling time vary according to the set-point.

However, comparing the results obtained with both controllers it can be appreciated that the adaptive controller provides less overshooting than the PI controller, indeed it is less than $14 \%$. The settling time increases to almost $1 s$. 


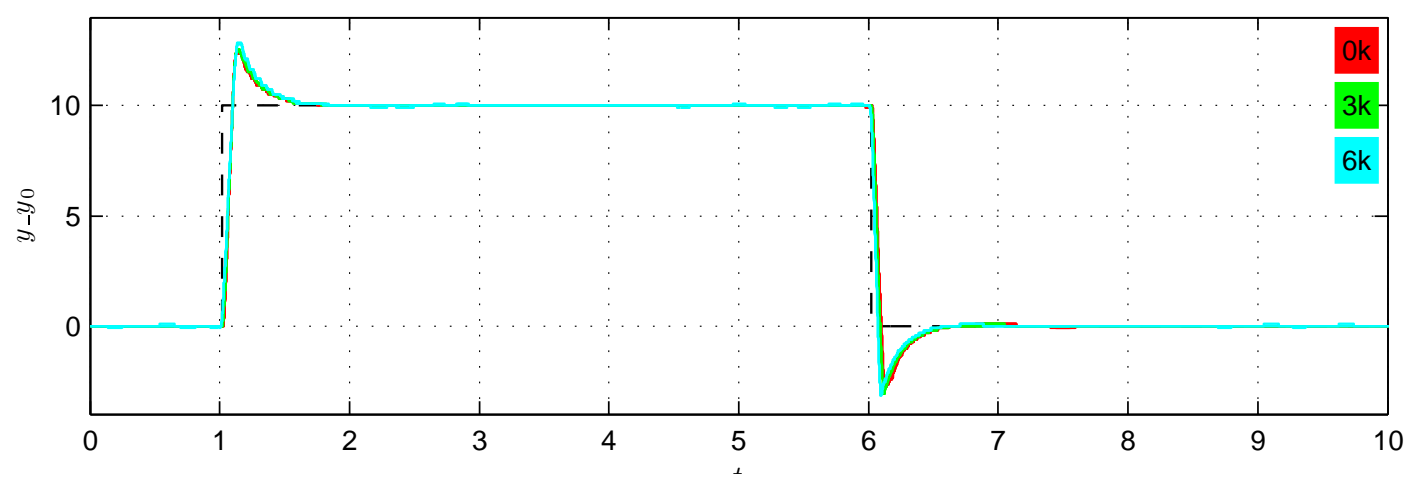

(a)

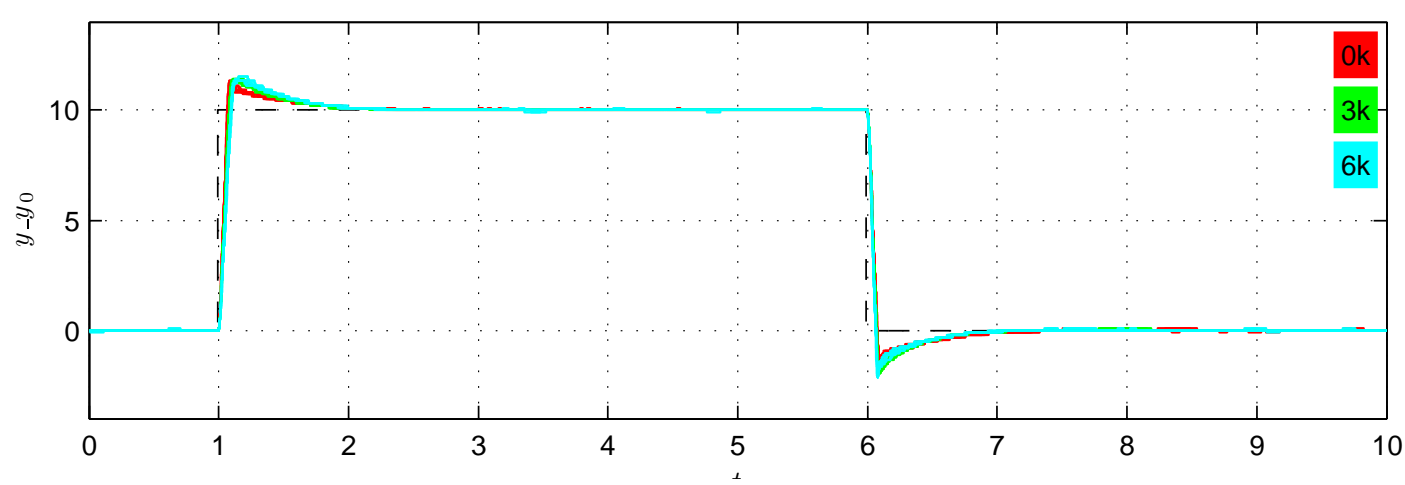

(b)

Figure 7.16: Step response of the system for different working set point.

\subsubsection{Experiment \# 2: Position Reference Tracking}

\section{- Objective}

Analyze the reference tracking capabilities of the controller, by evaluating the error between the command signal and the response of the system, and its dependency with the frequency of the input signal.

\section{- Description}

The controlled system is submitted to follow the trajectory: $y_{r}(t)=150+$ $125 \sin (w t) m m$, driving externals load of $m_{e x t}:=\{0,3,6\} \mathrm{kg}$, and frequencies $w \in[0.1,10] \mathrm{rad} / \mathrm{s}$.

- Data Processing 


\section{CONTROL STRATEGIES SHA}

- The signals obtained during experimentation are synchronized with the input signal at the biassed position.

- In order to quantify the dependency of the error in terms of the frequency of the input signal it is considered the maximum absolute error for data set, i.e. $|\tilde{y}(t)|_{\max }$.

\section{- Results}

- The response of the system to the desired trajectory $y_{r}(t)$ using the PI controller and the Adaptive controller driving different external load, are presented in Fig.7.17 and Fig. 7.18 respectively. As it is seen, both controller present good tracking characteristics, since the tracking error $\tilde{y}(t) \leq 1 \mathrm{~mm}$. It can also be appreciated that maximum of $\tilde{y}(t)$ corresponds with the change of direction on the piston motion. Even further, it can be appreciated an abrupt change in the error, that could be associated to stick-slip phenomena between the moving elements.

- The dependency of the error to the frequency of the input signal is depicted in Fig. 7.19. As it is to be expected, the error increases with the frequency of the signal input. However, the adaptive controller presents a bandwidth than the PI controller.

\subsubsection{Experiment \# 3: Robustness to external perturbations}

\section{- Objective}

Demonstrate the robustness of the system in face of external force perturbation.

\section{- Description}

The actuator is submitted to a sinusoidal input in closed loop. External force perturbation is performed by adding and removing weights during operation.

\section{- Data Processing}



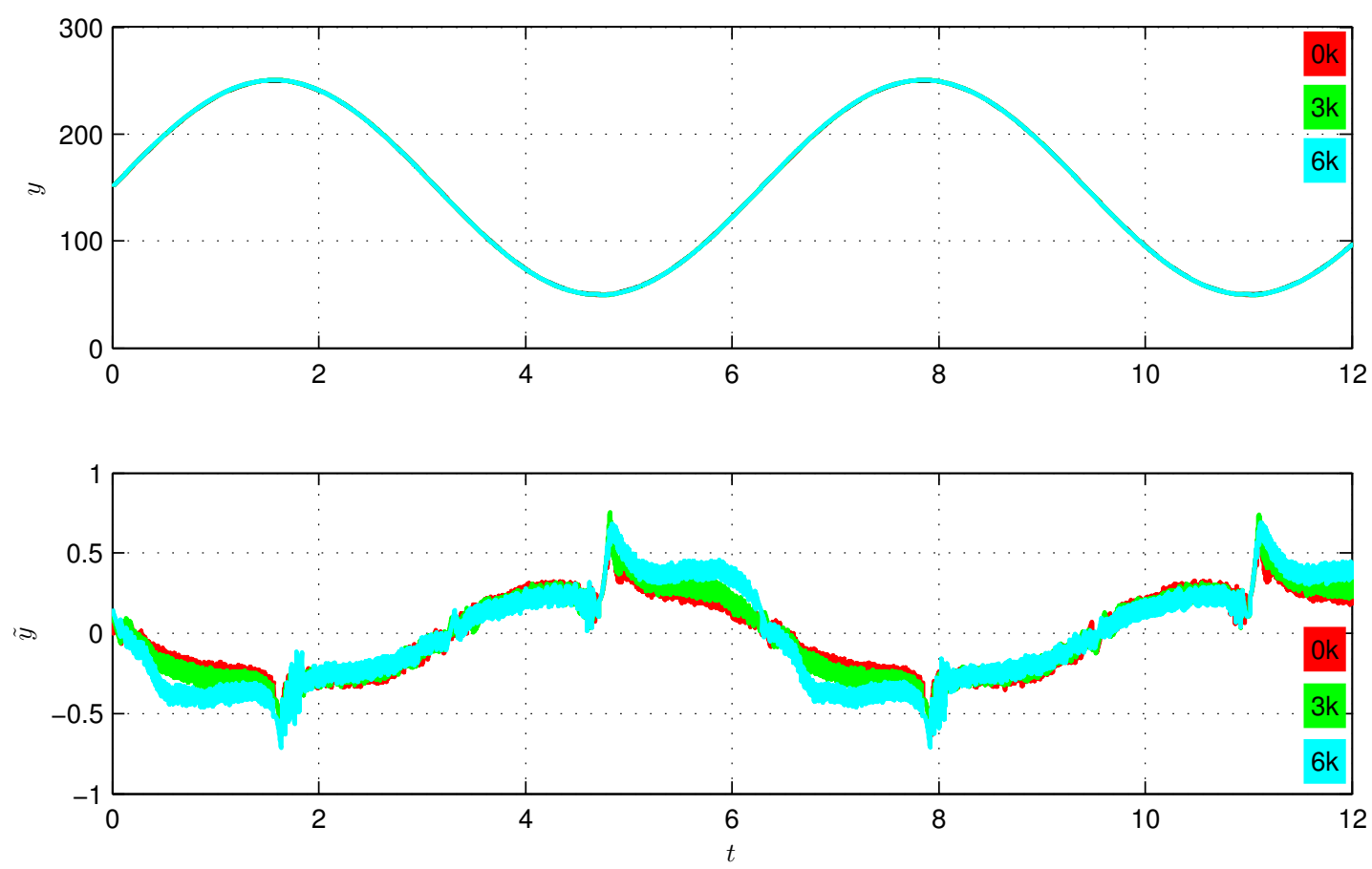

Figure 7.17: Reference tracking with PI controller.

\section{- Results}

The time response of the system with PI controller and Adaptive controller in front of external forces perturbation is presented in Fig. 7.20 and Fig. ?? respectively. In these charts, it is presented the position of the systems $y(t)$, the error tracking $\tilde{y}(t)$ and the measure of the force sensor $F(t)$. In both figures, the chart depicted at the bottom, it can be observed the action of the external mass (signaled as dashed grey vertical lines), and how it alter the force system of the SHA. As it can be observed on $y(t)$ and $\tilde{y}(t))$, these perturbations do not present significance on the output of the system. Nevertheless, in $\tilde{y}(t)$ for the adaptive controller, there is a very small perturbation due to the force loop of the hybrid controller, but once again it does not present a meaningful action to the output system. 


\section{CONTROL STRATEGIES SHA}
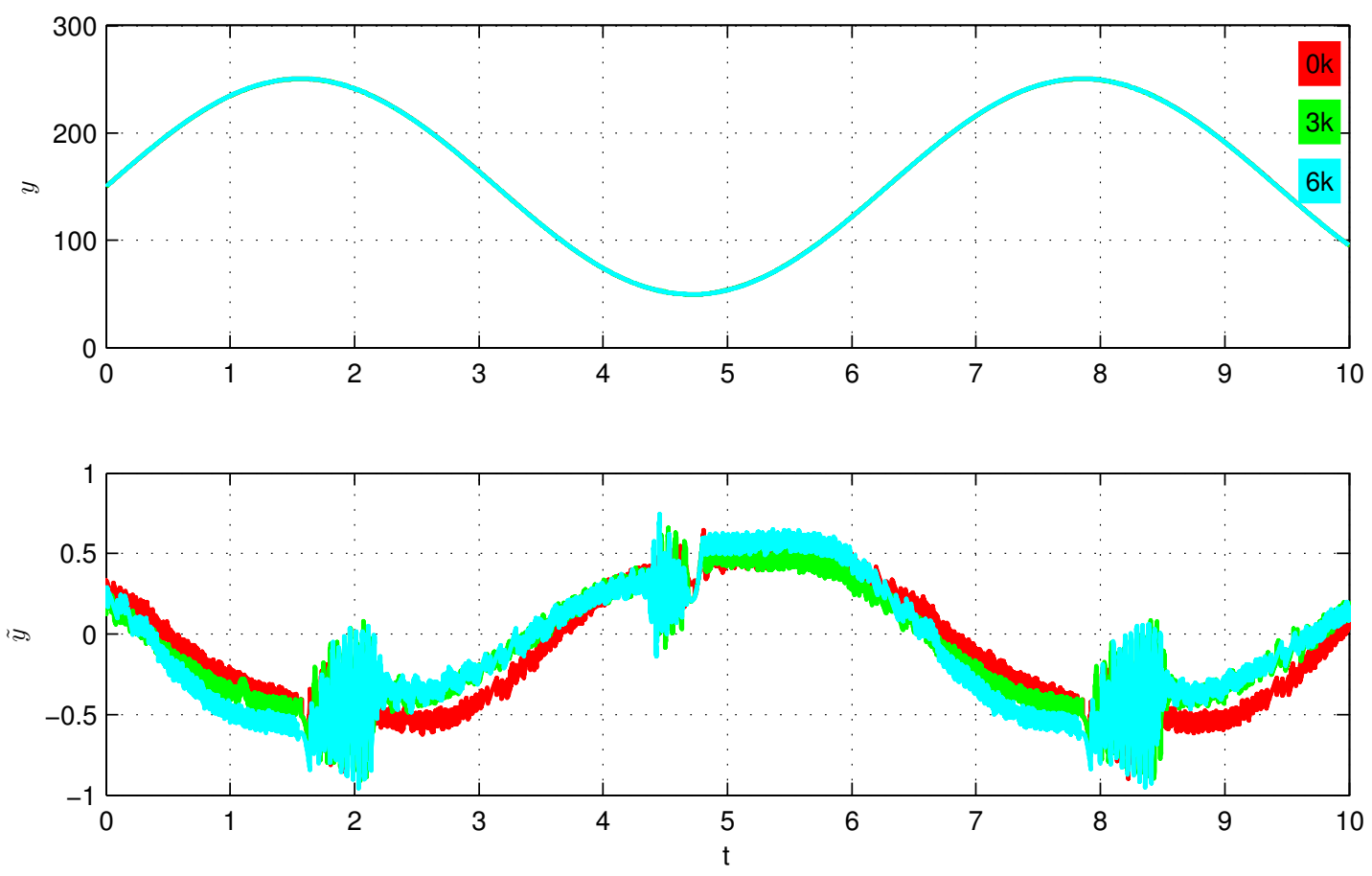

Figure 7.18: Reference tracking with Adaptive controller.

\subsection{Conclusions}

In this chapter is analyzed an adaptive force-position controller for the SHA.

The SHA model consists of two parts, one of them is associated with the manipulated variable and the other with the action of external forces.

If the model is simplified to just to the first part of the model, it is easy to fall into erroneous design of the controller, since the second part is directly coupled to the output affecting the steady state error.

In order to provide zero state error, it is needed a PI controller even though this controller adds another integrator to the closed-loop system. However, as it was proved it is possible to achieve robustness of the system if the controller is properly designed.

The tuning of a controller of each set-point of the SHA allows to keep the performance of the system in all its range of work. However, a new discussion arises when a switch of the controller is needed. Therefore, some parameters of the system must be 


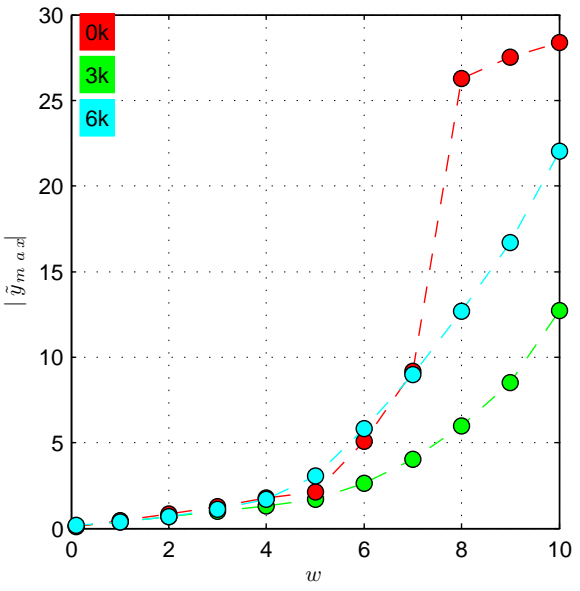

(a)

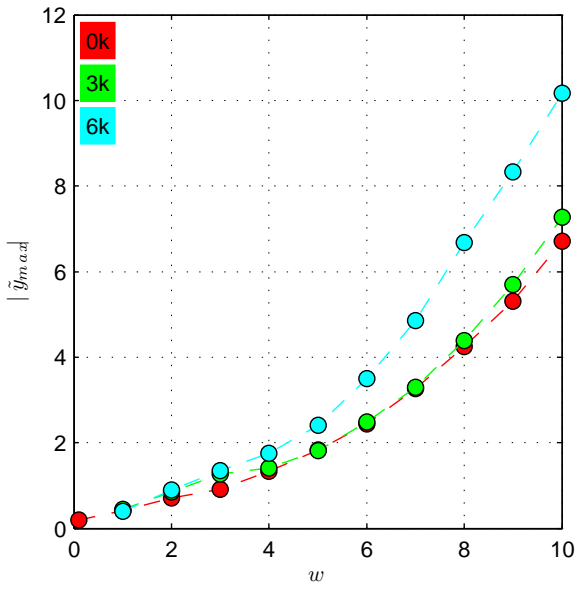

(b)

Figure 7.19: Frequency error dependency. 7.19(a): with PI controller. 7.19(b): with Adaptive controller.

estimated online in order to select the appropriate controller for current set-point. In order to provide smoothness and continuity on the controller parameters, their variation is conceived through splines.

The incorporation of a force loop to the control strategy enhance the performance of the controller, reducing the overshooting of the time response and improving its frequency response.

The adaptive force-position controller has proved to be robust during experimentation in face of external perturbation.

Even though experience indicates that control laws based on transfer functions do not provide the performance nor robustness of a nonlinear system, results shows that the adaptive controller presented in this chapter does provide them. 

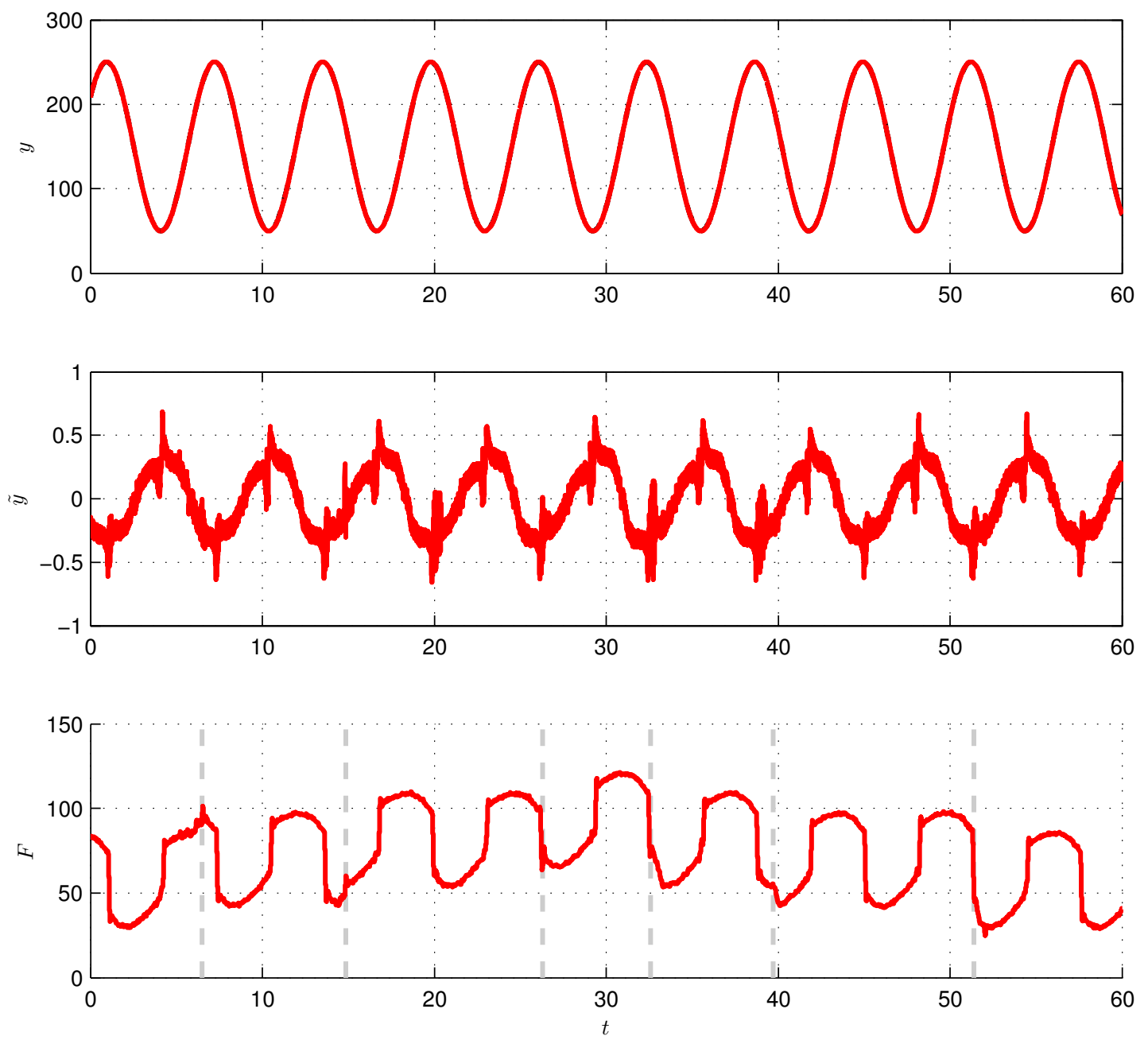

Figure 7.20: PI controller response under external perturbations. 

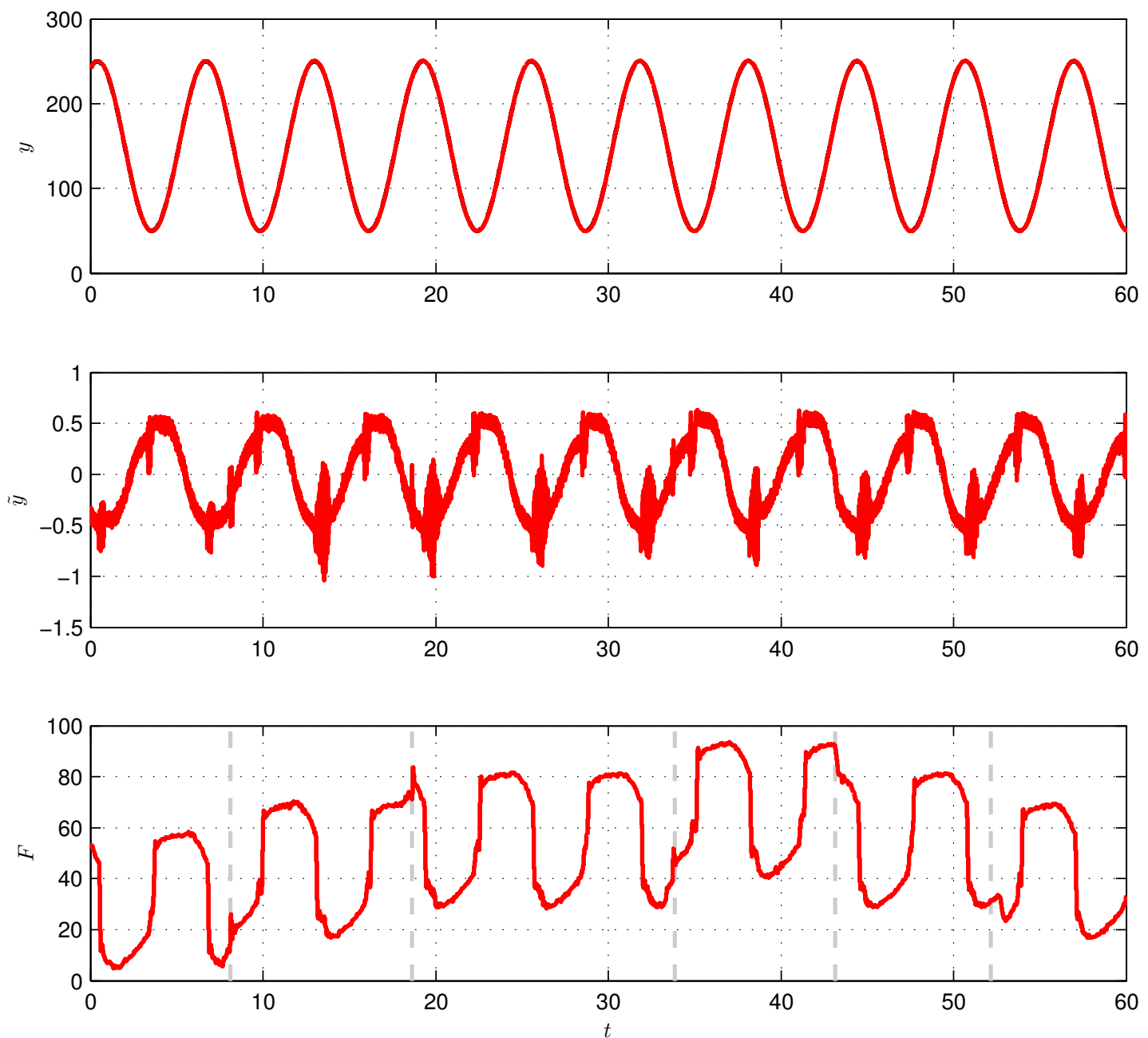

Figure 7.21: Adaptive controller response under external perturbations. 
7. CONTROL STRATEGIES SHA 


\section{Part III}

\section{Hydraulic Parallel Manipulator}





\section{8}

\section{Hydraulic 6 DoF Parallel Robot}

"... fear leads to anger, anger leads to hate, hate to suffering, I sense much fear in you..."

Master Yoda

\subsection{Introduction}

The Hydraulic 6DoF Parallel Robot is a 6UPS PM, powered by six of the Hydraulic Servo Actuator presented in Part II of this book.

The Hydraulic 6 DoF Parallel Robot was first conceived as the successor of the climbing robots framed within the TREPA program (21). The intention of this design was to overcame the power limitation that presented the previous versions of the climbing robot (8) (12), which were powered by electrical actuators.

The design of this former climbing robot was adapted for its implementation as a test-bed for advanced control strategies (22), where the main modification consisted on removing the claws and the re-allocation of the main hydraulic manifold. In this Chapter it is briefly introduced its mechatronic components of the hydraulic test-bed. 


\section{HYDRAULIC 6 DOF PARALLEL ROBOT}

\subsection{Description of the SG Mechanism}

\subsubsection{Mechanical Design}

The 6UPS parallel mechanism consists on two rings (upper ring and lower ring) connected by six legs, attached with a passive universal joint to the lower ring (U), and a passive spherical joint to the upper link (S). These legs are composed by two links coupled by a prismatic actuator $(\underline{\mathrm{P}})$, as shown in Fig.8.1. The position and orientation of the platform in space are prescribed by changing the length of the six legs.

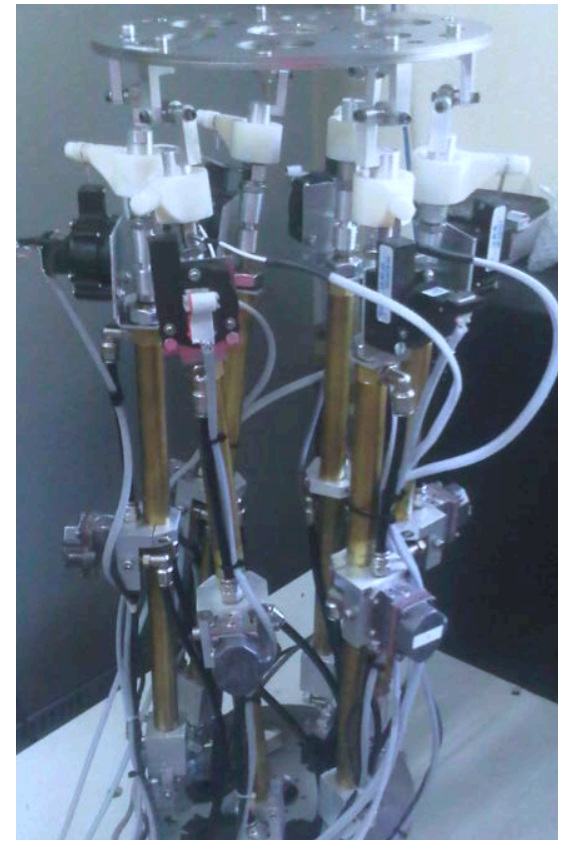

(a)

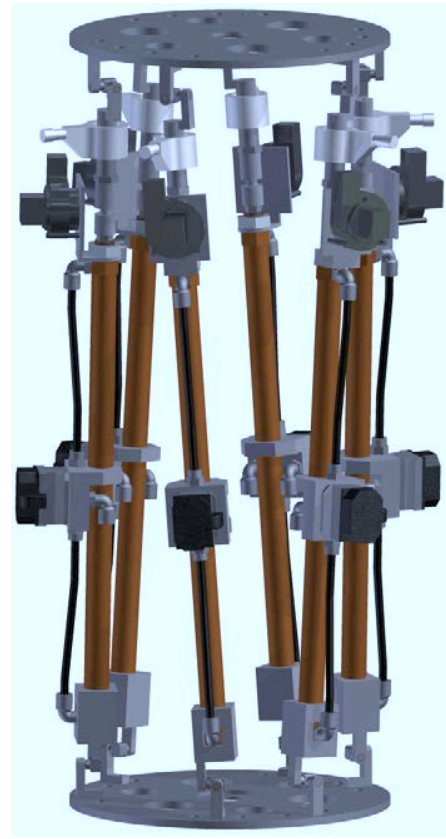

(b)

Figure 8.1: CAD of the 6UPS mechanism.

The design of a 6UPS parallel platform presents several alternatives that depends on the criteria adopted. For the former purpose as a climbing robot (21), the design of the mechanism was focused on the improvement of its workspace and dexterity, and dynamics, by keeping it as compact as possible. This consideration allowed that the mechanism could be easily adapted as a desktop test-bench.

In closed-loop kinematic chains, the workspace of the mechanism is strongly constrained by the range of work of the joints and the collision between the elements of the 
mechanism. For this particular mechanism the range of work of the universal joints and the spherical joints are critical for its workspace capabilities, therefore they must be enhanced. In particular, the range of work of the universal joints are vastly improved by considering $L$-shape elements which leads to the called open universal joints (12), instead of the traditional $U$-shape elements (see Fig. 8.2). A complete analysis of its range of work is detailed in (135) where the universal joint was the main element of the PM.

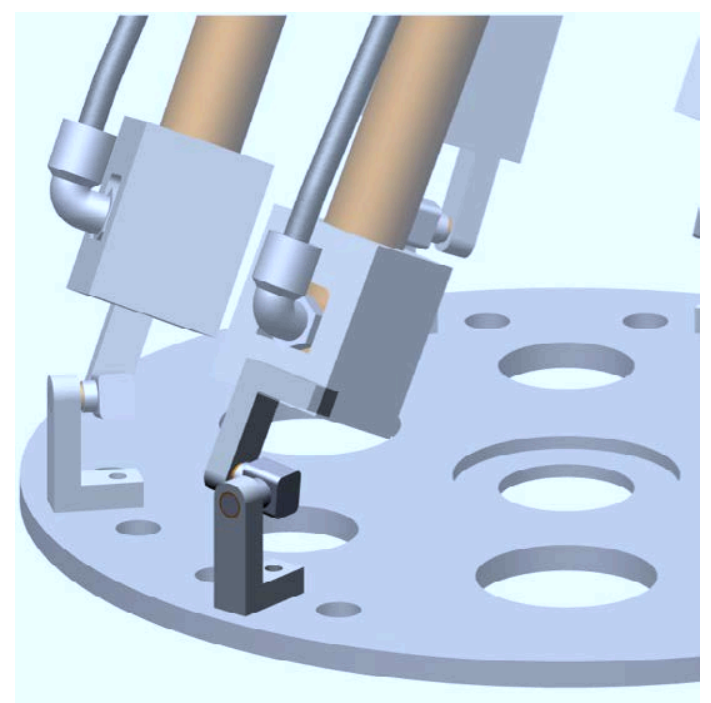

(a)

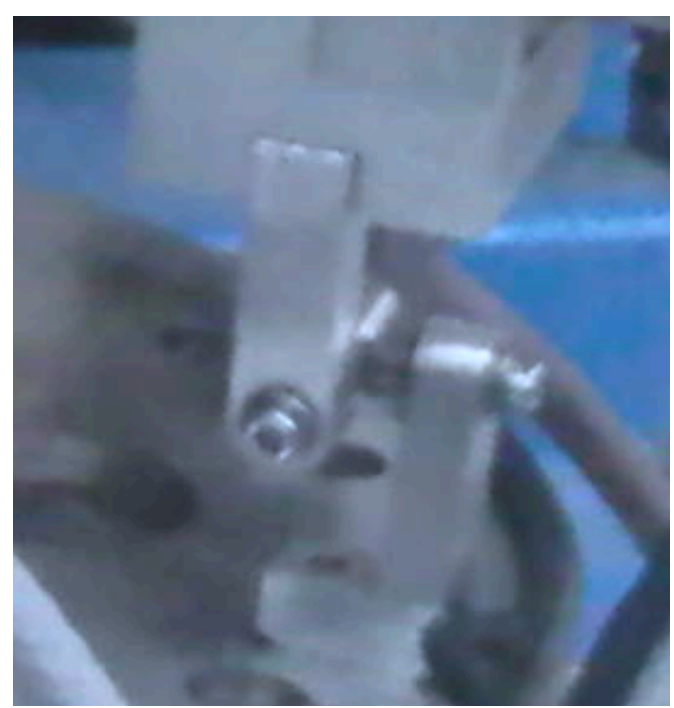

(b)

Figure 8.2: Universal joint details.

On the other hand, commercial spherical joints based on ball-socket assembly are expensive and their range of work are also limited. An alternative to ball-socket configuration is to combine an universal joint with a revolute joint whose axes are non-collinear and they intersects at a common point, which defines its center of rotation. The latter approach is commonly used in robotics (122) with the only precaution that during normal operation it must be avoided that axis of the revolute joint becomes co-llinear with any of the universal axes. Therefore, the the free rotation of the rod of the hydraulic actuator along its axis of action is taken into consideration along the universal joint, to create a virtual spherical joint (see Fig. 8.3). 


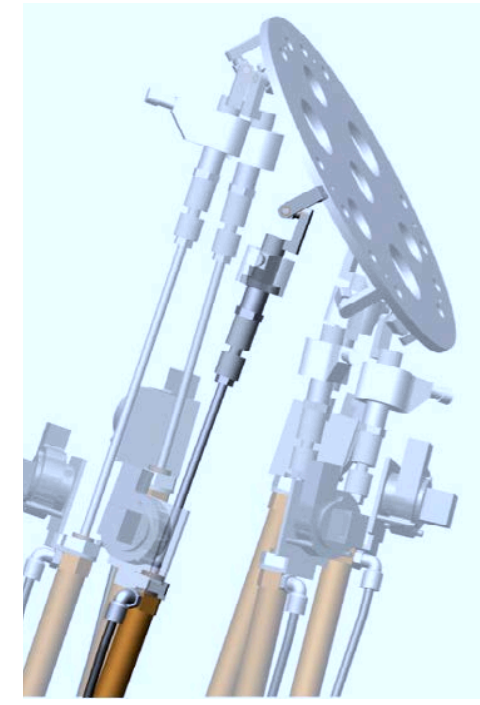

(a)

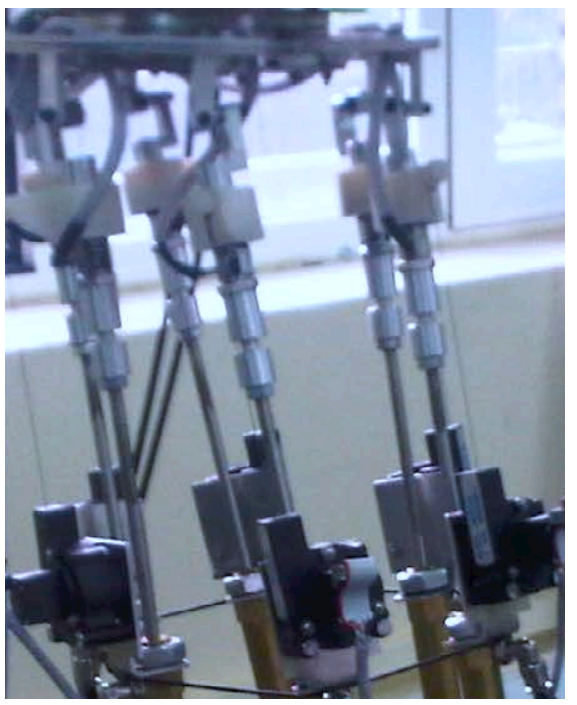

(b)

Figure 8.3: Spherical joint details.

The dimension of the elements of the mechanism are summed up in Table 8.1 (22).

Table 8.1: Elements of the 6UPS mechanism

\begin{tabular}{lccl} 
Element & Parameter & Size & Material \\
\hline \hline lower and upper ring & diameter & $190 \mathrm{~mm}$ & $6 \mathrm{~mm}$ steel plate \\
lower link & diameter & $20 \mathrm{~mm}$ & $300 \mathrm{~mm}$ inox cylinder \\
upper link & diameter & $6 \mathrm{~mm}$ & $300 \mathrm{~mm}$ inox rod \\
universal joint & aperture & $\pm 90^{\circ}$ & aluminum body - steel shaft \\
prismatic joint & stroke & $290 \mathrm{~mm}$ & \\
\hline
\end{tabular}

\subsubsection{Hydraulic Actuation System Design}

The PM has six hydraulic servo-actuator (detailed in Chapter 4), powered by a hydraulic power station. The latter one, consists of a positive displacement gear pump of $6.6 \mathrm{~cm}^{3} / \mathrm{min}$ driven by a electric motor of $1 H P$, providing $10 \mathrm{l} / \mathrm{min}$ at a maximum 
pressure of 40Bar (see Fig. 8.4(a)).

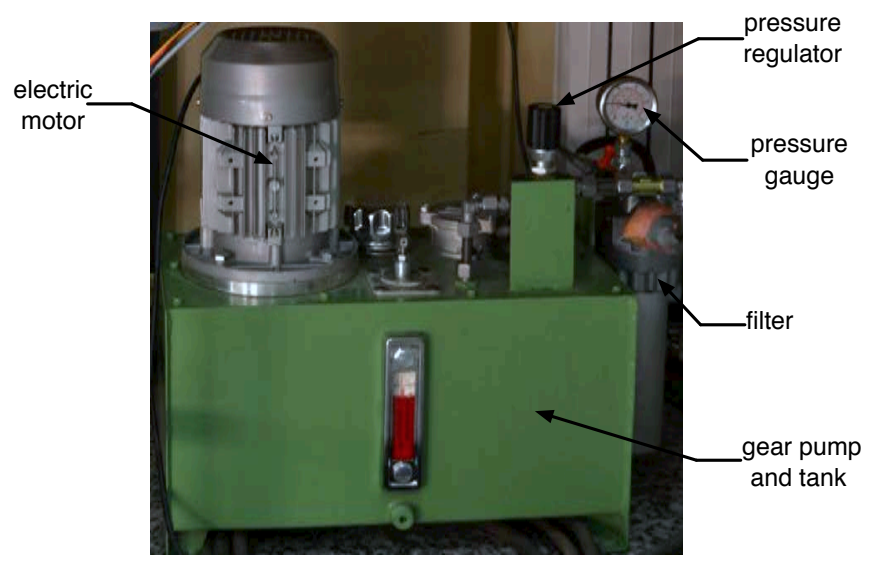

(a)

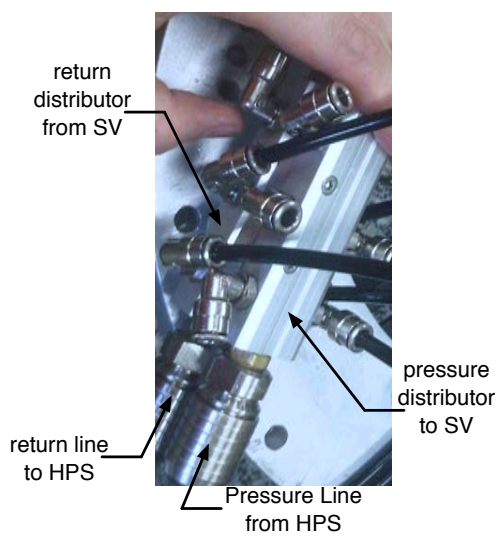

(b)

Figure 8.4: 8.4(a):Power Hydraulic Station. 8.4(b): flow distributor from/to the HPS and the SV.

The hydraulic flow is delivered to the six HSA using two monoblock distributors. One of them is connected to the pressure line and distributes the flow to the servovalves, and the other connects all the return lines of the servo-valve to the tank (see Fig. 8.4(b)).

\subsubsection{Electronics and Sensorization}

The main board of the open experimental platform is a dSPACE signal processor board which includes onboard $\mathrm{A} / \mathrm{D}$ and $\mathrm{D} / \mathrm{A}$ converters and a slave digital processor (DSP) that feeds the servovalve drivers.

The length and force exerted by all the prismatic actuator are measured by a LXEP40 linear encoder and a ELHS load cell respectively.

The ELHS load cell consists on a piezoresistive strain gage Wheatstone bridge, that provides $200 \mathrm{mV}$ full scale output. The sensor is provided with threads for tension and compression applications. The load cells are attached directly to the piston rod. It must be remarked, that this type of sensor only provide information of the net force applied in its extremes when one of extreme is grounded. In the case of this application, in order to get information of the net force, it must also take into account the net movement of the parts. 


\section{HYDRAULIC 6 DOF PARALLEL ROBOT}

The LXEP40 linear encoders provide continuous, precise position feedback with a resolution of $0.125 \mathrm{~mm}$, which for general purposes could be considered enough. Nevertheless, for accurate and low velocity movements this characteristic limits the overall performance of the positioning task. As it was mentioned before, a adaptive Kalman filter was implemented to overcome this constraint, which if could be diminished but not eliminated..

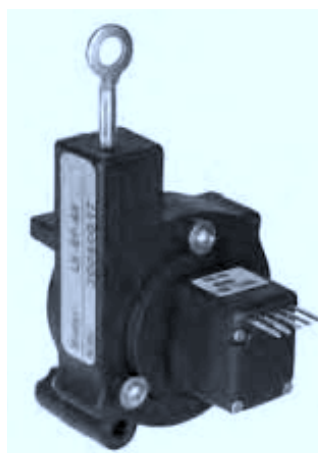

(a)

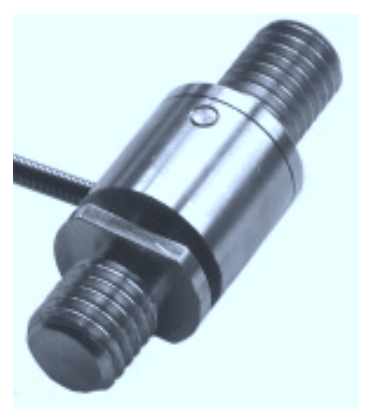

(b)

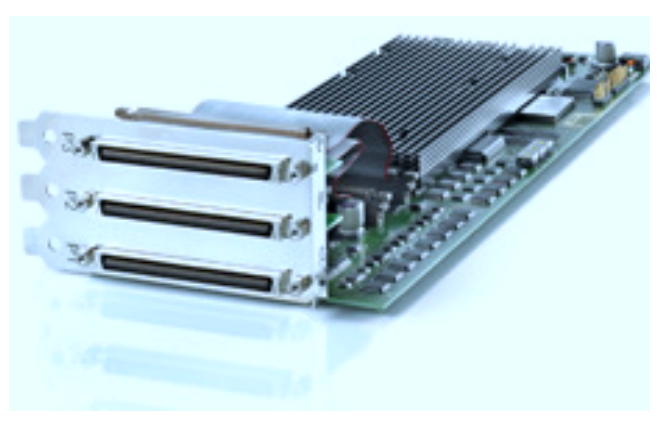

(c)

Figure 8.5

An 3-axis inertial measurement unit, is placed at the center of the upper ring, providing the orientation, angular velocity and angular acceleration.

\subsection{Simulation Environment}

The ADAMS environment is implemented to give support to the simulations where the control strategies are tested. More than 200 pieces that integrate the mechanism is imported in ADAMS, each one with its corresponding physical properties, i.e. mass and inertia, in order to have the more realistic results as possible.

The ADAMS environment is linked with Simulink, where it is incorporated the dynamic model of the hydraulic servo actuator presented in the second part of this book to drive each prismatic actuator defined in ADAMS. It is also incorporated the limitation of the encoders from the position sensors attached at each hydraulic cylinder. 


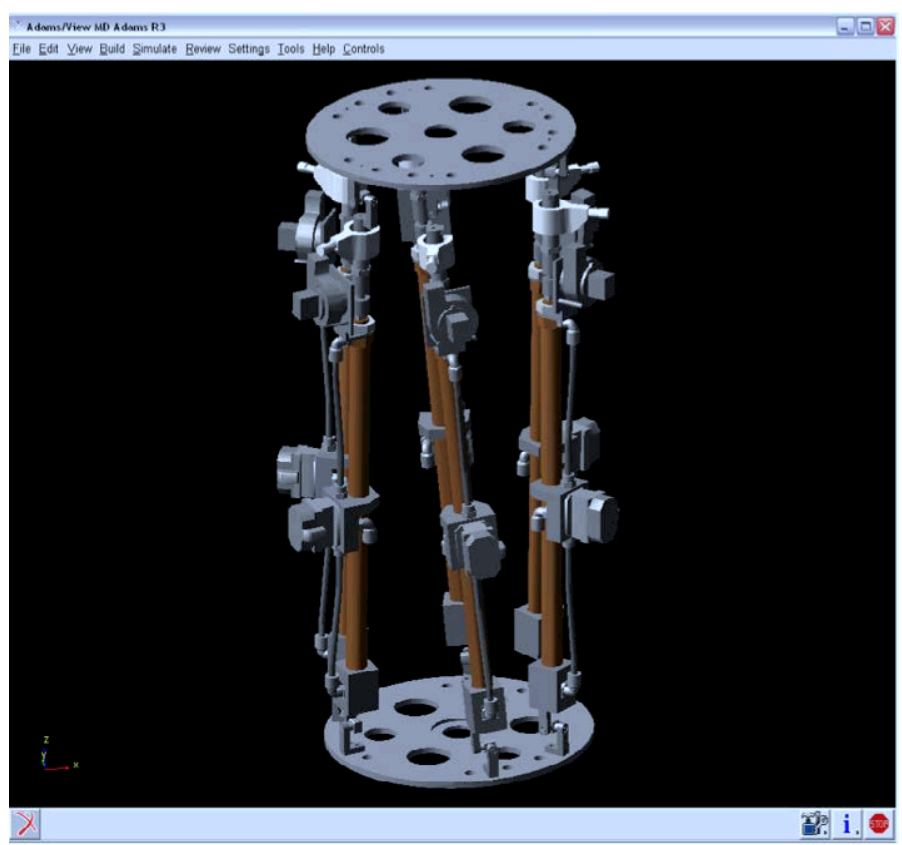

Figure 8.6: Simulation environment

\subsection{Conclusions}

The main parts of the parallel mechanism were introduced and some general considerations regarding the mechanical parts and sensors were discussed.

The detailed knowledge of the parts that integrate the mechanism under analysis has provided a requirement for the implementation of the simulator environment in order to provide results that could be easily taken to the real mechanism in the future. 
8. HYDRAULIC 6 DOF PARALLEL ROBOT 
9

\title{
Kinematic Analysis
}

\author{
"... what ever comes in our way, \\ what ever battle we have inside us, \\ we always have a choice... \\ it is the choice that makes us who we are, \\ and we can always chose to do what is right ..."
}

Peter Parker

\subsection{Introduction}

The solution of the direct kinematics (DK) of a PM is a complex problem due to the highly non-linear and coupled equations involved in the model. Several methods had been proposed: analytical, numerical, and based on redundant sensorization, among others.

In particular, the NR method was widely studied and implemented for finding the solution of the DK of PMs. Some of the variants of the NR method and their performance can be found in (100). Basically, the NR method searches the solution of a given function by successive approximations governed by its tangent. Therefore, the appropriate definition of the corresponding function it is crucial for its performance.

As the literature reveals, there exists several criteria for the formulation of this function. In (8) the DK of a 6 UPS PM is solved implementing a $49 \times 1$ constraint vector 


\section{KINEMATIC ANALYSIS}

that includes the holonomic constraints imposed by all the joints, the displacement of each actuator and the normalization of the Euler parameters. Even though this formulation presents a complete description of the kinematics of the PM, the calculation of its Jacobian it is not straight forward. On the other hand, a scalar function for each leg derived from the vector loop-closure equation is implemented in (69) for a 6 PSU PM, and each element of the Jacobian matrix is obtained by taking the partial derivative of the corresponding scalar function with respect to the corresponding unknown variable. In (49), the DK of an octahedral Stewart-Gough type platform is obtained with the NR method and the definition of a kinematically equivalent 3-legged mechanism. Another example is (159), where the position problem of a planar $3 \underline{R R R}$, is based on a constraint vector that depends on which joint is sensorized and whether extra sensors are used. All these formulations are specific for each mechanism, then their adaptation for other mechanism is very difficult and in some cases impossible.

\subsection{Inverse Kinematics}

As in most of the parallel mechanisms, the inverse kinematic modeling can be achieved by a simple observation of its geometry. Let consider the schematic diagram presented in Fig.9.1(a). Therefore the length of each leg is given by:

$$
L_{i}=\left\|{ }^{O} \boldsymbol{r}+{ }^{O} R_{P}{ }^{P} \boldsymbol{b}_{i}-{ }^{O} \boldsymbol{a}_{i}\right\|
$$

where ${ }^{O} R_{P}$ is the relative orientation between the reference frames $P_{u v w}$ and $O_{x y z}$ expressed as a rotation matrix. ${ }^{P} \boldsymbol{b}_{i}$ is the localization of the anchoring of the i-limb on the moving platform expressed on the reference frame $P_{u v w} .{ }^{O} a_{i}$ in the localization of the anchoring of the i-limb on the fixed platform expressed on the reference frame $O_{x y z}$.

\subsection{Workspace}

The workspace of the mechanism is defined by all the positions and orientations that it can achieve without exceeding its physical limitations, which are given by the implicit constraints of the kinematic chains, such as the range of work of each joints and the collisions between the elements of the mechanism, among others. In this work, 


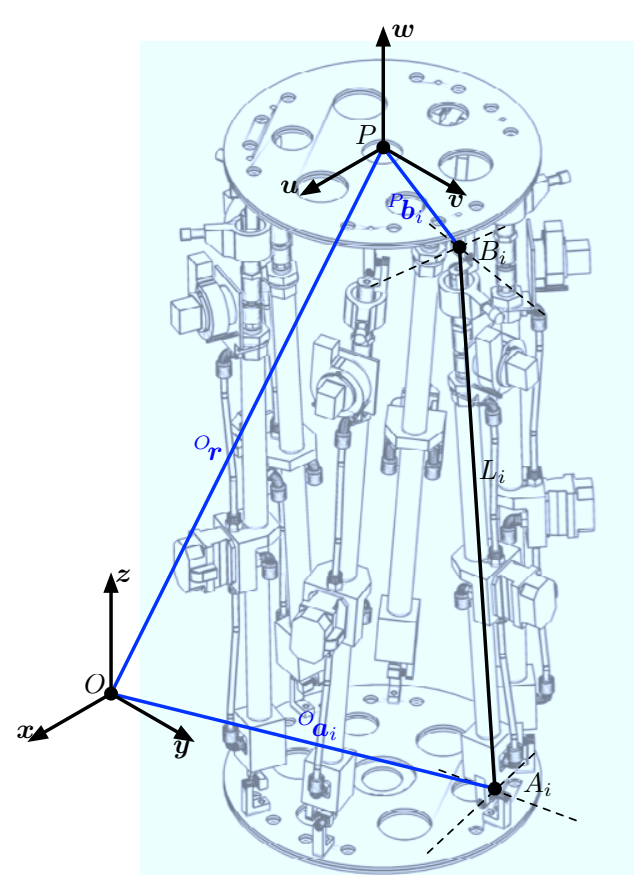

(a)

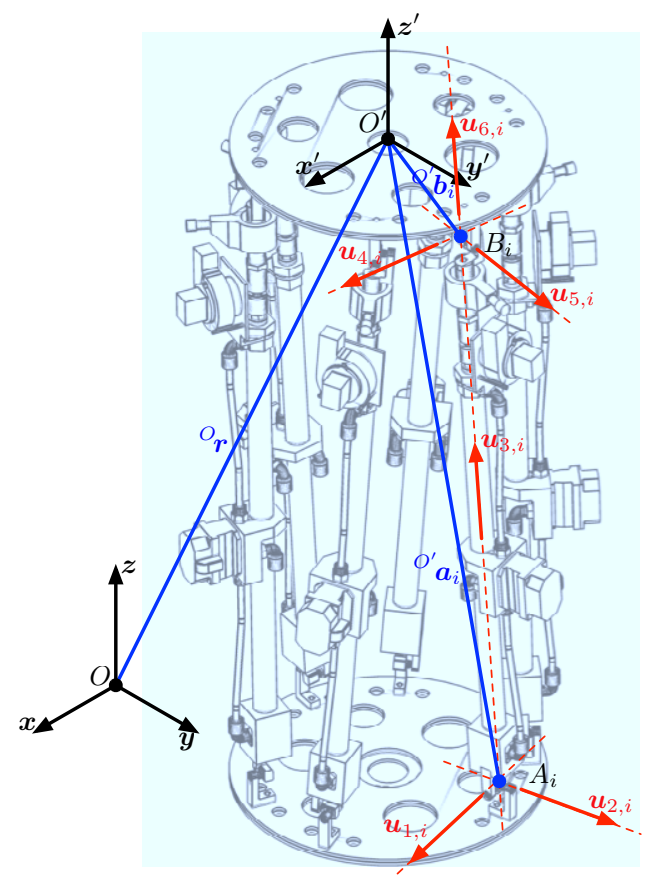

(b)

Figure 9.1: Schematic diagram of the mechanism $6 \mathrm{UPS}$.

the evaluation of the physical limitation of the parallel mechanism follows the same formulation that (135), which considers:

1. Prismatic Stroke Constraint: the prismatic stroke constraint imposed on each leg can be written as $L_{\min } \leqslant d_{i} \leqslant L_{\max }$. Where $L_{i}$ is the state of the prismatic joint of the i-limb given by (9.1), and $L_{\min }$ and $L_{\max }$ are the minimum and maximum stroke of the actuator.

2. Universal and Spherical Joint Constraint: it is considered that its relative movement can never exceed the region limited by the circular cone defined by an axis normal to the joint, an aperture $\delta_{\max }$ and its apex located at the center of the joint (111).

3. Leg Interference: it is based on the evaluation of the minimum distance between two cylinders (99). In this work, it is only considered the interference between the limbs of the mechanism. 


\section{KINEMATIC ANALYSIS}

The simpler way to obtain the workspace of a mechanism is to propose states for the end effector and verify if the geometric limitations are met. Therefore, a grid $W_{q}$ composed by all the nodes $\boldsymbol{x}_{i}=\left[x_{1}, x_{2}, \cdots, x_{n}\right]$ is defined, where $n=6$ are the degrees of freedom of the mechanism, and each elements is bounded according to: $x_{j_{\min }} \leqslant x_{j} \leqslant$ $x_{j_{\text {max }}}$, for $j=1,2, \cdots, 6$ and $i=1,2, \cdots, m . m=\prod_{j=1}^{m}\left(x_{j_{\text {max }}}-x_{j_{\text {min }}}\right) / \Delta_{j}$ is the number of nodes to evaluate and $\Delta_{j}$ is the increment for each element.

Given that the 6UPS mechanism is a $6 \mathrm{DoF}$ spatial mechanism, in this work three different workspace representation for the mechanism are presented and detailed.

In Fig.9.2 it is presented the dexterous workspace of the mechanism as a solid volume. This representation shows all the positions that the moving platform can reach performing Roll - Pitch - Yaw rotations up to $\pm 25^{\circ}$.

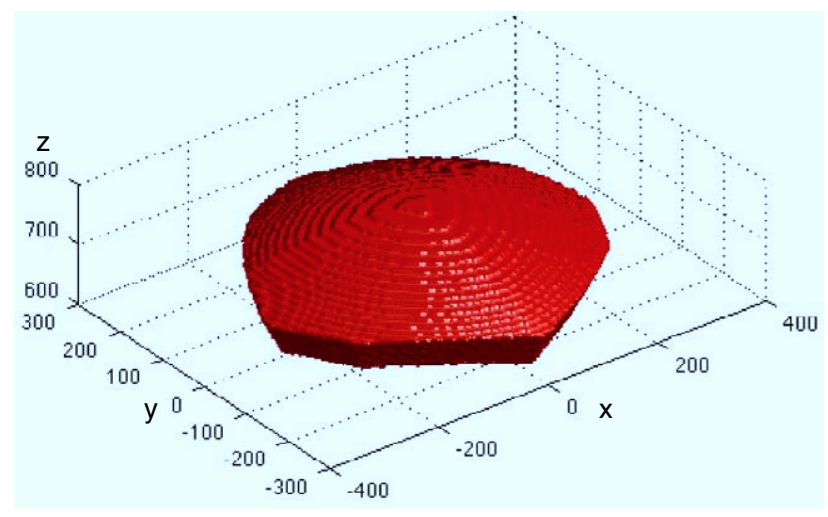

Figure 9.2: $6 \mathrm{U} \underline{\mathrm{PS}}$ maximum dexterous workspace

The maximum pure translation can be obtained from the slices of the workspace presented in Fig.9.3(a) and Fig.9.3(b). As it can be seen, at $z=675 \mathrm{~mm}$ the mechanism can achieve maximum pure translation of $\pm 300 \mathrm{~mm}$ along the $\boldsymbol{x}$ and $\boldsymbol{y}$ axis. From Fig.9.3(b) it is observed that the workspace poses a $100 \mathrm{~mm}$ thick shell type geometry.

The maximum translation workspace of the moving platform for a fixed orientation is presented in Fig. 9.4. This representation corresponds with the moving platform always parallel to the fixed platform, i.e. Roll - Pitch $-Y a w=\left[0^{\circ}, 0^{\circ}, 0^{\circ}\right]$.

The workspace still conserves the shell type geometry, however its thickness is almost $200 \mathrm{~mm}$ for all the configurations (see Fig.9.5(b)), and the maximum pure translation at plane $z=600$ is $\pm 500 \mathrm{~mm}$. 


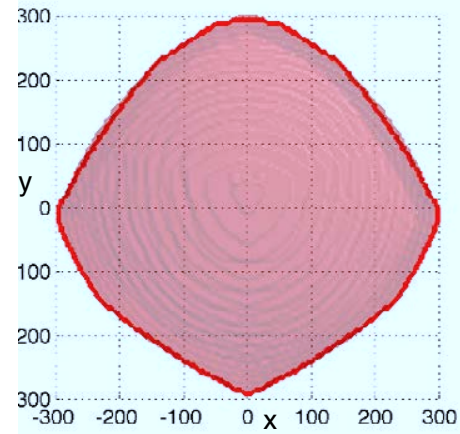

(a)

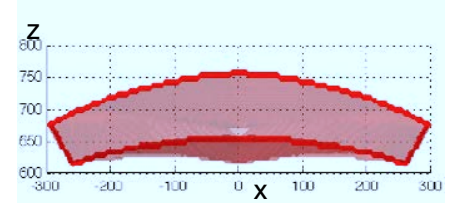

(b)

Figure 9.3: Maximum pure translation. 9.3(a): slice of the workspace with plane $z=675$. 9.3(b): slice of the workspace with plane $y=0$.

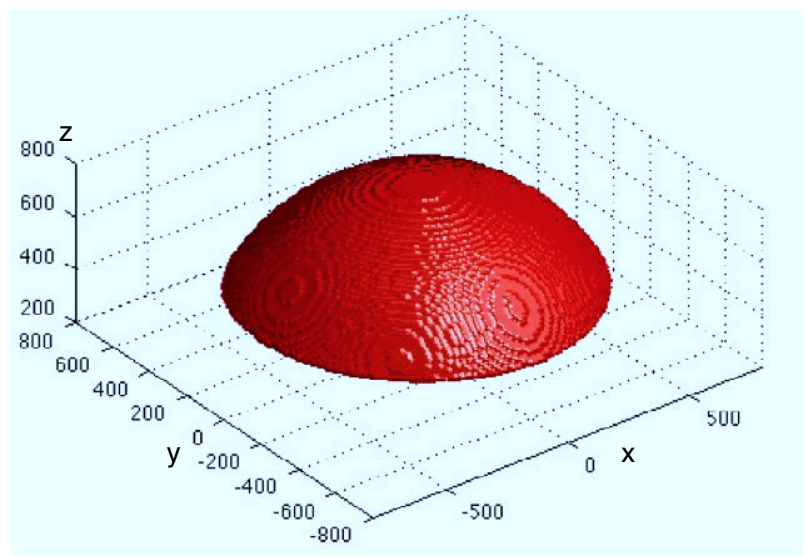

Figure 9.4: 6 UPS maximum pure translations workspace representation with both platform parallel.

The maximum orientation workspace for a fixed position $\boldsymbol{r}=[0,0,700]$ of the moving platform is presented in Fig.9.6.

From $9.7(\mathrm{a})$ and $9.7(\mathrm{~b})$ it can be seen that the maximum pure rotation of the mechanism are $\pm 55^{\circ}$ for Pitch and Roll, and $\pm 115^{\circ}$ for $Y a w$. 


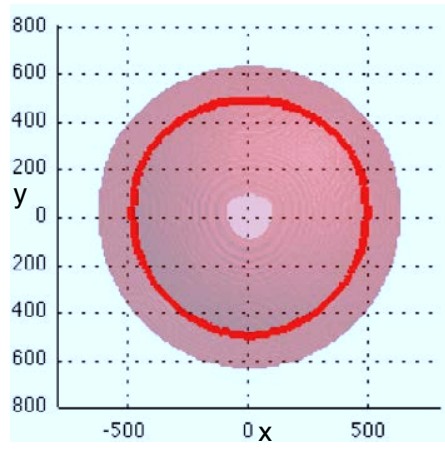

(a)

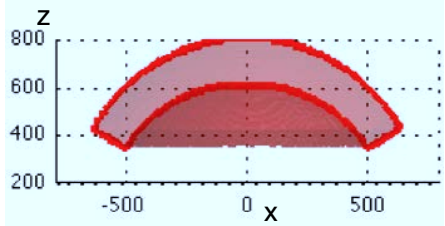

(b)

Figure 9.5: $6 \mathrm{UPS}$ maximum pure translations workspace representation with both platform parallel. 9.5(a): slice of the workspace with plane $z=600$. 9.5(b): slice of the workspace with plane $y=0$.

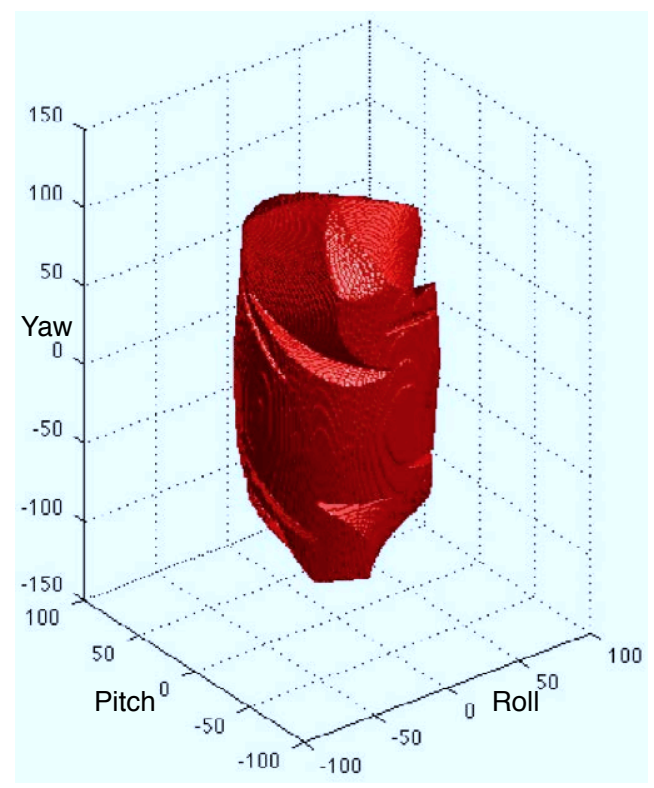

Figure 9.6: 6 U $\underline{P S}$ maximum rotation workspace representation. Solid volume representation.

\subsection{Direct Kinematics}

The methodology proposed in this work consists on the definition of a distance constraint vector $\boldsymbol{\Phi}(\boldsymbol{q})=\mathbf{0}$ of the mechanism using the multibody formulation and the 


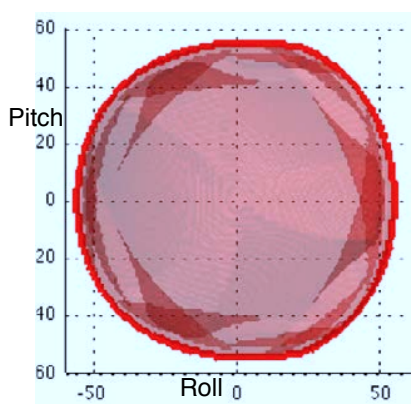

(a)

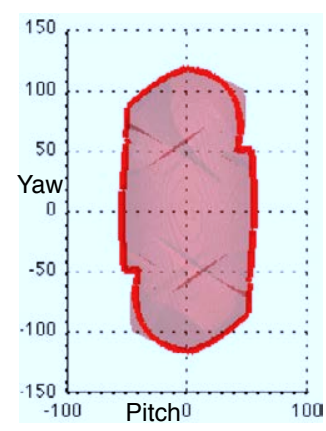

(b)

Figure 9.7: 6 ULS maximum rotation workspace representation. 9.7(a): slice of the workspace with plane $Y a w=0^{\circ} .9 .7(\mathrm{~b})$ : slice of the workspace with plane $R o l l=0^{\circ}$.

implementation of the successive approximation method of NR.

\subsubsection{Direct Kinematics Approach}

A mechanism is composed by several rigid bodies that are interconnected by joints. Its configuration can be described in terms of the generalized coordinates of its elements, which are affected by the kinematics constraints of the mechanism. A kinematic constraint between two bodies imposes the conditions on the relative motion between them, and it must imply the geometry of the joint that couples these elements. A mechanism with $n$ holonomic kinematic constraints can be expressed as (66):

$$
\boldsymbol{\Phi}(\boldsymbol{q})=\left[\begin{array}{c}
\phi_{1}(\boldsymbol{q}) \\
\vdots \\
\phi_{n}(\boldsymbol{q})
\end{array}\right]=\mathbf{0}
$$

where $\boldsymbol{q}$ is the generalized coordinate vector of the mechanism.

It is important to remark that the definition of the kinematic constraints $\phi_{i}(\boldsymbol{q})$ that compose the constraint vector $\boldsymbol{\Phi}(\boldsymbol{q})$ are not unique and it is directly related with the complexity of the Jacobian matrix. Therefore, in this work a generic distance constraint function is used for each leg that provides a simple calculation of the Jacobian matrix whose elements represent geometric characteristics of the PM.

The numerical method proposed proceeds as follows (see Fig. 9.8):

1. The constraint vector of the mechanism is calculated. 


\section{KINEMATIC ANALYSIS}

2. If the constraint vector satisfies the error condition (i.e. $\left\|\mathbf{\Phi}\left(\boldsymbol{q}_{k}\right)\right\|<\epsilon$ ), then the method finds a solution and the configuration of the mechanism is given by $\boldsymbol{q}_{k}$. If not, the method proceeds with the iterative calculation.

3. The Jacobian matrix $\boldsymbol{\Phi}_{\boldsymbol{q}}\left(\boldsymbol{q}_{k}\right)$ is calculated.

4. A new estimation for the configuration of the mechanism $\boldsymbol{q}_{k+1}$ is approximated implementing the Newton-Raphson method with the Jacobian matrix $\boldsymbol{\Phi}_{\boldsymbol{q}}\left(\boldsymbol{q}_{k}\right)$.

5. The new estimation is employed to calculate the constraint vector in the following iteration.

As it is often common with numerical methods, the methodology proposed may not converge. Therefore, a forced exit is generated if the method finds no solution after 100 iterations.

It must be noticed that the norm of $\boldsymbol{p}$ must always equals one, thus, after the new estimation is performed in step 4, its norm is forced to be one by considering $\boldsymbol{p}_{k+1}=\boldsymbol{p}_{k+1} /\left\|\boldsymbol{p}_{k+1}\right\|$.

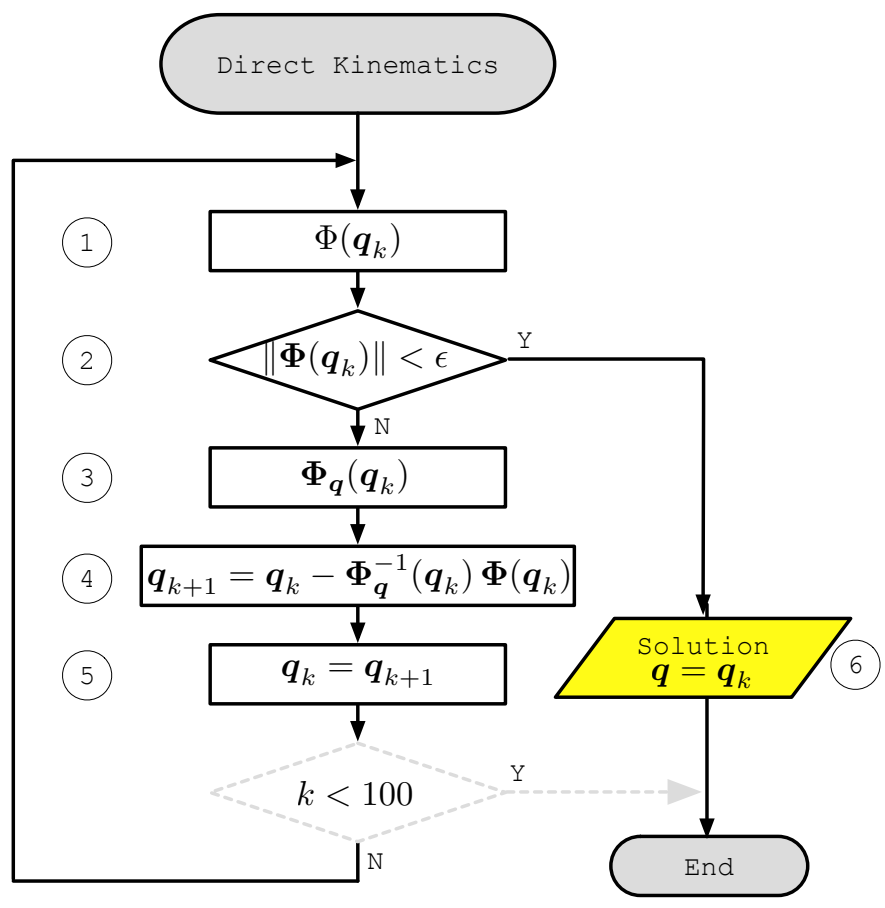

Figure 9.8: Direct Kinematics Algorithm. 


\subsubsection{Constraint vector definition}

Taking into consideration a generic PM with $n$ legs composed by two links, let us define a fixed reference frame $O_{x y z}$ and let us attach a moving frame $P_{u v w}$ in the moving platform of the PM as shown in Fig. 9.9. The location and orientation of the moving platform is given by the generalized coordinate vector: $\boldsymbol{q}=[\boldsymbol{r}, \boldsymbol{p}]$, where $\boldsymbol{r}=[x, y, z]^{T}$ defines its position and $\boldsymbol{p}=\left[e_{0}, e_{1}, e_{2}, e_{3}\right]^{T}$ defines its orientation in terms of the Euler parameters (i.e. quaternion representation). Euler parameters are used in this methodology since they not only provide a compact and non-singular representation for orientation, but also they provide useful properties and identities for the manipulation of rotations.

Independently of the PM's topology, the following vectorial equation must be met for the $n$ legs of the mechanism:

$$
\overrightarrow{O A_{i}}+\overrightarrow{A_{i} B_{i}}+\overrightarrow{B_{i} C_{i}}=\overrightarrow{O_{i} P_{i}}+\overrightarrow{P_{i} C_{i}}
$$

Without loosing generality, let say that the length of $\overrightarrow{B_{i} C_{i}}$ remains invariant for all the configurations of the mechanism, i.e. $\left\|\overrightarrow{B_{i} C_{i}}\right\|=l_{0 i}$. Therefore, for all the postures of the mechanism (i.e. $\forall \boldsymbol{q}$ ) a distance constraint function for the $i t h$ leg can be defined as follows:

$$
\phi_{i}(\boldsymbol{q})=\left\|\boldsymbol{l}_{i}(\boldsymbol{q})\right\|-l_{0 i}=0
$$

where $\boldsymbol{l}_{i}(\boldsymbol{q})=\overrightarrow{O A_{i}}+\overrightarrow{A_{i} B_{i}}-\overrightarrow{O_{i} P_{i}}-\overrightarrow{P_{i} C_{i}}$. The latter one, can be expressed as:

$$
\boldsymbol{l}_{i}(\boldsymbol{q})={ }^{O} \boldsymbol{a}_{i}+\boldsymbol{b}_{i}-\left({ }^{O} \boldsymbol{r}+{ }^{O} \boldsymbol{R}_{P}(\boldsymbol{p}){ }^{P} \boldsymbol{c}_{i}\right),
$$

where ${ }^{O} \boldsymbol{R}_{P}(\boldsymbol{p})$ is the orientation of the end effector expressed as a rotation matrix (hereafter ${ }^{O} \boldsymbol{R}_{P}$ for simplification). Considering the constraint distance function given by (9.4) for the $n$ legs and arranging them into a single vector, the constraint vector (9.2) for a generic PM can be stated as,

$$
\boldsymbol{\Phi}(\boldsymbol{q})=\left[\begin{array}{c}
\left\|\boldsymbol{l}_{1}(\boldsymbol{q})\right\|-l_{01} \\
\vdots \\
\left\|\boldsymbol{l}_{n}(\boldsymbol{q})\right\|-l_{0 n}
\end{array}\right]=\mathbf{0} .
$$




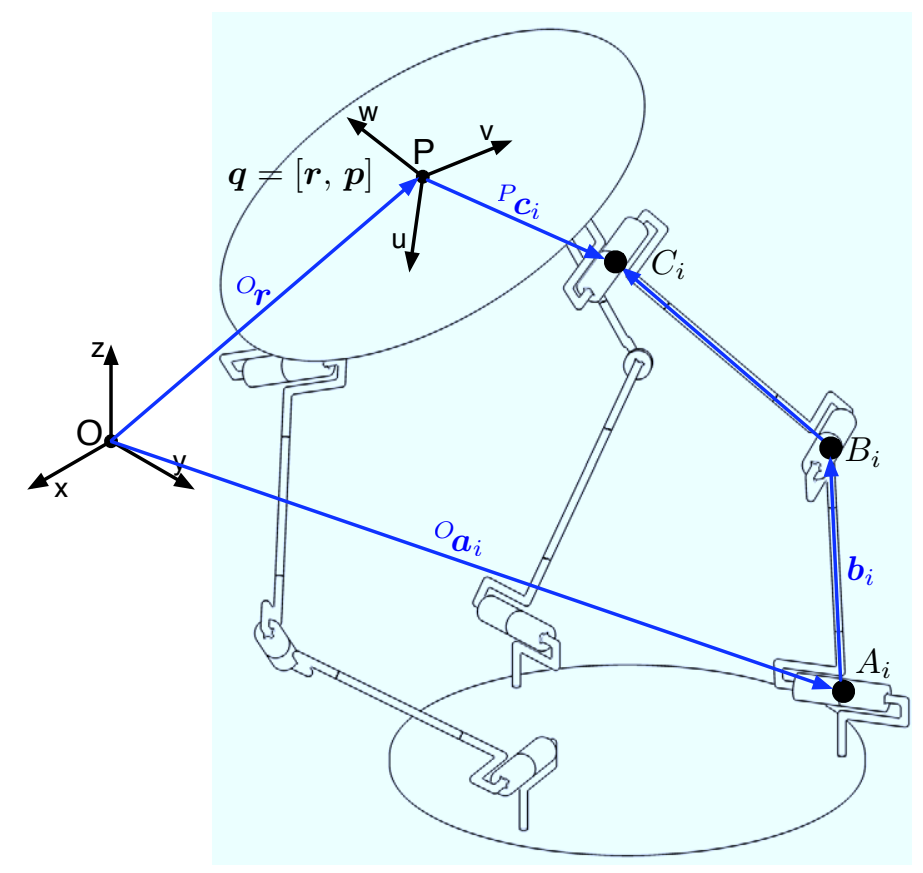

Figure 9.9: Schematic diagram of a two-links leg of a generic PM.

\subsubsection{Jacobian matrix of the constraint vector}

Let us remember that the generalized coordinate vector of the end effector was defined as $\boldsymbol{q}=[\boldsymbol{r}, \boldsymbol{p}]$. Therefore, the Jacobian matrix $\boldsymbol{\Phi}_{\boldsymbol{q}}(\boldsymbol{q})$ of the constraint vector $\boldsymbol{\Phi}(\boldsymbol{q})$ can be found as follows,

$$
\boldsymbol{\Phi}_{\boldsymbol{q}}(\boldsymbol{r}, \boldsymbol{p})=\left[\begin{array}{cc}
\frac{\partial}{\partial \boldsymbol{r}}\left[\phi_{1}(\boldsymbol{r}, \boldsymbol{p})\right] & \frac{\partial}{\partial \boldsymbol{p}}\left[\phi_{1}(\boldsymbol{r}, \boldsymbol{p})\right] \\
\vdots & \vdots \\
\frac{\partial}{\partial \boldsymbol{r}}\left[\phi_{n}(\boldsymbol{r}, \boldsymbol{p})\right] & \frac{\partial}{\partial \boldsymbol{p}}\left[\phi_{n}(\boldsymbol{r}, \boldsymbol{p})\right]
\end{array}\right]
$$

In order to simplify the notation, let us define the following vector $\boldsymbol{u}_{i}$ as follows:

$$
\boldsymbol{u}_{i}={ }^{O} \boldsymbol{a}_{i}+\boldsymbol{b}_{i}-\left({ }^{O} \boldsymbol{r}+{ }^{O} \boldsymbol{R}_{P}{ }^{P} \boldsymbol{c}_{i}\right) .
$$

The derivate of the constraint function with respect to the position of the end 
effector can be found as follows,

$$
\begin{aligned}
\frac{\partial}{\partial \boldsymbol{r}}\left[\phi_{i}(\boldsymbol{r}, \boldsymbol{p})\right] & =\frac{\partial}{\partial \boldsymbol{r}}\left[\left\|\boldsymbol{l}_{i}(\boldsymbol{r}, \boldsymbol{p})\right\|-l_{0 i}\right] \\
& =\frac{\partial}{\partial \boldsymbol{r}}\left[\sqrt{\boldsymbol{u}^{T} \boldsymbol{u}}-l_{0 i}\right] \\
& =\underbrace{\frac{1}{2 \sqrt{\boldsymbol{u}^{T} \boldsymbol{u}}} 2 \boldsymbol{u}_{i}}_{\hat{\boldsymbol{u}}} \underbrace{\frac{\partial}{\partial \boldsymbol{r}}\left[\boldsymbol{u}_{i}\right]}_{\boldsymbol{I}_{3 x 3}} \\
& =\hat{\boldsymbol{u}}_{i} .
\end{aligned}
$$

In the same way, the derivative of the constraint function with respect to the orientation of the end effector is obtained according to the following operations,

$$
\begin{aligned}
\frac{\partial}{\partial \boldsymbol{p}}\left[\phi_{i}(\boldsymbol{r}, \boldsymbol{p})\right] & =\frac{\partial}{\partial \boldsymbol{p}}\left[\left\|\boldsymbol{l}_{i}(\boldsymbol{r}, \boldsymbol{p})\right\|-l_{0 i}\right] \\
& =\frac{\partial}{\partial \boldsymbol{p}}\left[\sqrt{\boldsymbol{u}^{T} \boldsymbol{u}}-l_{0 i}\right] \\
& =\underbrace{\frac{1}{2 \sqrt{\boldsymbol{u}^{T} \boldsymbol{u}}} 2 \boldsymbol{u}_{i}}_{\hat{\boldsymbol{u}}} \frac{\partial}{\partial \boldsymbol{p}}\left[\boldsymbol{u}_{i}\right] \\
& =\hat{\boldsymbol{u}}_{i}\left(-\frac{\partial}{\partial \boldsymbol{p}}\left[{ }^{O} \boldsymbol{R}_{P}{ }^{P} \boldsymbol{c}_{i}\right]\right) \\
& =\hat{\boldsymbol{u}}_{i}\left(-2{ }^{O} \boldsymbol{R}_{P}{ }^{P} \tilde{\boldsymbol{c}}_{i} \boldsymbol{G}\right),
\end{aligned}
$$

where ${ }^{P} \tilde{\boldsymbol{c}}_{i}$ is the skew antisymmetric matrix of ${ }^{P} \boldsymbol{c}_{i}=\left[\begin{array}{lll}c_{i x}, & c_{i y}, & c_{i z}\end{array}\right]^{T}$, given by:

$$
P_{\tilde{\boldsymbol{c}}_{i}}=\left[\begin{array}{ccc}
0 & -c_{i z} & c_{i y} \\
c_{i z} & 0 & -c_{i x} \\
-c_{i y} & c_{i x} & 0
\end{array}\right],
$$

and $\boldsymbol{G}=\left[\boldsymbol{e},-\tilde{\boldsymbol{e}}+e_{0} \boldsymbol{I}\right]$ is an identity matrix (see C).

Then, the Jacobian matrix of the constraint vector for a generic PM can be stated as the following $n \times 7$ matrix:

$$
\boldsymbol{\Phi}_{\boldsymbol{q}}(\boldsymbol{q})=\left[\begin{array}{cc}
\hat{\boldsymbol{u}}_{1} & -2 \hat{\boldsymbol{u}}_{1}{ }^{O} \boldsymbol{R}_{P}{ }^{P} \tilde{\boldsymbol{c}}_{1} \boldsymbol{G} \\
\vdots & \vdots \\
\hat{\boldsymbol{u}}_{n} & -2 \hat{\boldsymbol{u}}_{n}{ }^{O} \boldsymbol{R}_{P}{ }^{P} \tilde{\boldsymbol{c}}_{n} \boldsymbol{G}
\end{array}\right] .
$$

As it was mentioned above, the method is governed by the inverse of the Jacobian matrix $\left(\boldsymbol{\Phi}_{\boldsymbol{q}}\right)$. However, since $\boldsymbol{\Phi}_{\boldsymbol{q}}$ is not square, its inverse can not be directly calculated and the Moore-Penrose pseudo-inverse is implemented instead. 


\section{KINEMATIC ANALYSIS}

\subsubsection{Performance Evaluation}

The performance of the methodology is quantified in terms of the following key performance indexes (KPI):

1. Convergence: it measures the ratio of convergence independently of the result, and it is defined as:

$$
C \%=\frac{\sum_{j=1}^{n_{W S}} n_{C}}{n_{W S}} \times 100,
$$

where $n_{W S}$ are the nodes of the workspace evaluated and $n_{C}$ are the nodes where the method finds a solution in less than 100 iteration.

2. Accuracy: it evaluates the overall error of the method. It is defined as the ratio of the solutions that satisfy a predefined error tolerance and the nodes where it converges, in other words:

$$
A_{c c i} \%=\frac{\sum_{k=1}^{n_{n c}} n_{\delta_{i}}}{\sum_{j=1}^{n_{W S}} n_{C}} \times 100,
$$

where $n_{\delta_{i}}$ are the nodes of the workspace that satisfy the error tolerance. For this work two error tolerances are defined:

$$
\begin{array}{ll}
\delta_{1}: & \delta_{P}<1 \times 10^{-6} \mathrm{~mm}, \delta_{O}<0.01^{\circ}, \\
\delta_{2}: & \delta_{P}<1 \times 10^{-3} \mathrm{~mm}, \delta_{O}<0.1^{\circ},
\end{array}
$$

where $\delta_{P}$ and $\delta_{O}$ are the errors in position and orientation respectively (the orientation error is defined in the next paragraphs). During the evaluation, it is also obtained the maximum $\left(\delta_{O M}\right.$ and $\left.\delta_{P M}\right)$, the mean $\left(\overline{\delta_{O}}\right.$ and $\left.\overline{\delta_{P}}\right)$ and the standard deviation $\left(\sigma_{\delta_{O}}\right.$ and $\left.\sigma_{\delta_{P}}\right)$ of the error in position and orientation, respectively.

3. Velocity: the number of iterations needed until the method finds a solution is considered as a measure of velocity. This measure eliminates the dependability on the hardware where the simulation are carried on. The maximum $\left(i_{M}\right)$, average $(\bar{i})$ and standard deviation $\left(\sigma_{i}\right)$ of the number of iterations are taken as performance indexes. 
The constraint function proposed for the DK is evaluated for all the configurations of the workspace of thePM, following the procedure depicted in Fig. 9.10 and detailed in the following paragraphs:

1. For a given configuration $\boldsymbol{q}=[\boldsymbol{r}, \boldsymbol{p}]$ of the PM that belongs to its workspace, the joint states $\boldsymbol{Q}$ is obtained by means of the calculation of the inverse kinematics (IK) of the PM.

2. An initial estimation $\boldsymbol{q}_{0}=\left[\boldsymbol{r}_{0}, \boldsymbol{p}_{0}\right]$ for the end effector is randomly generated (detailed bellow).

3. The state of the end effector $\boldsymbol{q}_{D}=\left[\boldsymbol{r}_{D}, \boldsymbol{p}_{D}\right]$ is obtained using the numerical method presented in section 9.4, considering the initial estimation generated $\boldsymbol{q}_{0}$ and the joint states $\boldsymbol{Q}$.

4. If the method converges (i.e. $\exists \boldsymbol{q}_{D}$ ), the error of the solution found is calculated as follows:

$$
\begin{array}{r}
\left\|\boldsymbol{r}-\boldsymbol{r}_{D}\right\|<\delta_{P}, \\
\left\|[\phi, \theta, \psi]-\left[\phi_{D}, \theta_{D}, \psi_{D}\right]\right\|<\delta_{O},
\end{array}
$$

where the vector $[\phi, \theta, \psi]$ is the orientation in Roll-Pitch-Yaw representation.

The initial estimation $\boldsymbol{q}_{0}=\left[\boldsymbol{r}_{0}, \boldsymbol{p}_{0}\right]$ is generated by considering initial distances of $L_{E r r_{0}}=1,10,25,50 \mathrm{~mm}$ and orientation of $\theta_{e r r_{0}}=1,10,25,50$, of the real value randomly placed.

Thus, the initial estimation for the position $\boldsymbol{r}=\left[r_{x}, r_{y}, r_{z}\right]$ is defined as follows:

$$
\boldsymbol{r}_{0}=\left[r_{x} \pm L_{e r r_{0}}, r_{y} \pm L_{e r r_{0}}, r_{z} \pm L_{e r r_{0}}\right],
$$

where the sign \pm is randomly selected.

The generation of the initial estimation for the orientation is more complex. The real orientation $\boldsymbol{p}$ is expressed in the angle-axis representation $(\theta, \boldsymbol{v})$, where $\boldsymbol{v}$ is a unit vector associated with the Euler axis of rotation and $\theta$ is the magnitude of rotation. These elements are modified with a $\theta_{e r r_{0}}$ distance as follows: 


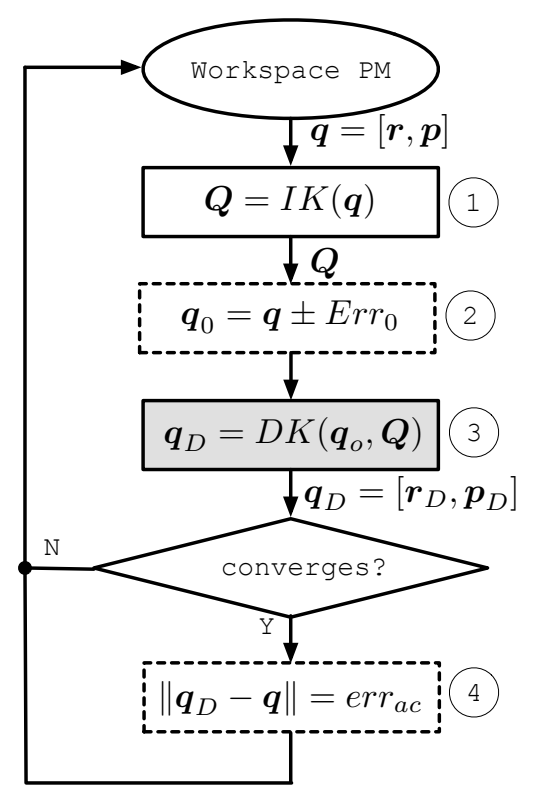

Figure 9.10: Evaluation Procedure (2 and 4 are symbolic expression).

$$
\begin{aligned}
& \theta_{0}=\theta \pm \theta_{e r r_{0}}, \\
& \boldsymbol{v}_{0}=\boldsymbol{R}_{E r r 0 x} \boldsymbol{R}_{E r r 0 y} \boldsymbol{v},
\end{aligned}
$$

where $\boldsymbol{R}_{E r r 0 x}$ and $\boldsymbol{R}_{E r r 0 y}$ are pure rotation of $\pm \theta_{e r r_{0}}$ along the $\boldsymbol{x}, \boldsymbol{y}$ axes, respectively. The sign \pm is randomly selected. Then, the new orientation $\left(\theta_{0}, \boldsymbol{v}_{0}\right)$ is expressed again as a quaternion (i.e: $\left.\left(\theta_{0}, \boldsymbol{v}_{0}\right) \rightarrow \boldsymbol{p}_{0}\right)$.

Since the home posture $\boldsymbol{q}_{H}$ is always a reference configuration of any manipulator, it is also considered as an initial estimation.

The exploration is defined by discretized intervals for each coordinate that describes the state of the end effector (i.e., X, Y, Z, $\psi, e_{i}$ ).

The results obtained during simulations are summed up in Table 9.1. The columns labeled as $\boldsymbol{q}_{*}$ presents the results for the initial estimation.

As it can be seen in Table 9.1, the worst rate of convergence $(C \%)$ found is $84.45 \%$, which corresponds with a considerably poor initial estimation (i.e. $\boldsymbol{q}_{50}$ for the 6 UPS PM). However, for better initial estimations (i.e. $\boldsymbol{q}_{1}, \boldsymbol{q}_{10}, \boldsymbol{q}_{25}$ ), the rate of convergence is above the $98 \%$. 
Table 9.1: Performance evaluation of the method.

\begin{tabular}{|c|c|c|c|c|c|c|}
\hline $\mathrm{PM}$ & index & $q_{H}$ & $q_{1}$ & $q_{10}$ & $q_{25}$ & $q_{50}$ \\
\hline $6 \mathrm{UPS}$ & $C \%$ & 100 & 99.98 & 99.93 & 98.89 & 84.45 \\
\hline \multirow{8}{*}{$\left(5838767^{*}\right)$} & $i_{M}$ & 8 & 32 & 40 & 100 & 100 \\
\hline & $\bar{i} \pm \sigma_{i}$ & $6.2 \pm 0.4$ & $5.4 \pm 0.7$ & $5.7 \pm 0.7$ & $6.2 \pm 0.8$ & $7.2 \pm 2.7$ \\
\hline & $\delta_{O M}$ & $1.110^{-6}$ & 130.7 & 137.7 & 276.5 & 283.4 \\
\hline & $\overline{\delta_{O}} \pm \sigma_{\delta_{O}}$ & $(2.0 \pm 5) 10^{-8}$ & $0.006 \pm 0.8$ & $0.01 \pm 1.2$ & $0.23 \pm 5$ & $6.6 \pm 29$ \\
\hline & $\delta_{P M}$ & $6.5510^{-6}$ & 125 & 1390 & 1444 & 1520 \\
\hline & $\overline{\delta_{P}} \pm \sigma_{\delta P}$ & $(6.2 \pm 15) 10^{-7}$ & $0.006 \pm 1.2$ & $0.01 \pm 3.1$ & $0.8 \pm 28$ & $15.8 \pm 116$ \\
\hline & $A c c_{1} \%$ & 99.62 & 99.20 & 99.92 & 98.44 & 79.58 \\
\hline & $A c c_{2} \%$ & 100 & 99.97 & 99.67 & 98.66 & 79.76 \\
\hline
\end{tabular}

It can be observed from the maximum errors found (i.e. $\delta_{O M}$ and $\delta_{P M}$ ) that the outcome of the calculation may provide an erroneous solution. However, by examining the mean value and the standard deviation of the errors, it can be seen that it does not occur frequently, and they are highly dependent on the initial estimation (a typical characteristic of numerical methods). This is clearly shown in the results obtained for the initial estimations $\boldsymbol{q}_{1}$ and $\boldsymbol{q}_{10}$, where the index $A c c_{1}$ reveals that the method provides an accuracy of $1 \times 10^{-6} \mathrm{~mm}$ and $1^{\circ} \times 10^{-2}$ above the $90 \%$ of all the simulation. Even more, if the performance index Acc 2 is considered, it can be seen that $97 \%$ of convergences guarantee an accuracy of $1 \times 10^{-3} \mathrm{~mm}$ and $0.1^{\circ}$.

From Table 9.1 it is also observed that in a worst case scenario a solution is found in 100 iterations $\left(i_{M}=100\right)$. However, the average of the iterations and their standard deviations demonstrate that this is an unlikely case. Indeed, it can be seen that the method can provide a solution in less than 10 iterations (see columns $\boldsymbol{q}_{1}$ and $\boldsymbol{q}_{10}$ for index $\bar{i}$.

\subsection{Instantaneous Kinematics}

The instantaneous kinematics of a parallel mechanism is given by the relation between the joint velocity space $\dot{\boldsymbol{q}}$ and the end effector velocity space $\dot{\boldsymbol{x}}$, stated as follows:

$$
J_{x} \dot{\boldsymbol{x}}=J_{q} \dot{\boldsymbol{q}}
$$

where $J_{x}$ is often known as the direct jacobian matrix, and $J_{q}$ is the inverse jacobian matrix.

The traditional process of finding the instantaneous kinematics of a parallel mechanism consists in differentiating the inverse kinematic equation. However, this process is 


\section{KINEMATIC ANALYSIS}

tedious and may lead to possible errors. A much better methodology is the use of screw theory and the concept of reciprocal screws (158). They provide a better geometrical insight into the problem and allow the precise and complete analysis of singularities of the mechanism (122).

Lets consider the twists associated to the joints of the i-limb of the 6UPS mechanism as shown in Fig.9.1(b). According to (145), the resulting twist for the end effector $\hat{\$}_{e f}$ can be found as:

$$
\begin{aligned}
\hat{\$}_{e f} & =\left[\begin{array}{c}
\boldsymbol{\omega} \\
\boldsymbol{v}_{o}
\end{array}\right] \\
& =\dot{q}_{1, i} \hat{\$}_{1, i}+\dot{q}_{2, i} \hat{\Phi}_{2, i}+\cdots+\dot{q}_{6, i} \hat{\Phi}_{6, i}
\end{aligned}
$$

Where $\hat{\$}_{j, i}$ is the unitary j-twist associated to the j-joint of the i-limb, and $\dot{q}_{j, i}$ is the intensity of the $\hat{\$}_{j, i}$. $\boldsymbol{\omega}$ is the angular velocity and $\boldsymbol{v}_{o}$ is the linear velocity.

Using the property of reciprocal screws, all the twists of the passive joints can be eliminated from (9.24). The reciprocal screw to any spherical and universal joint, is a $\$_{0}$ screw that passes throw their centers. In particular, the reciprocal screw for each limb of the 6 UPS mechanism is given by

$$
\$_{r, i}=\left[\begin{array}{c}
\boldsymbol{u}_{3, i} \\
\boldsymbol{b}_{i} \times \boldsymbol{u}_{3, i}
\end{array}\right]
$$

where $\boldsymbol{u}_{3, i}=\overline{A_{i} B_{i}} /\left\|\overline{A_{i} B_{i}}\right\|$ is the direction vector associated to the $\mathrm{i}$-limb.

Taking the reciprocal product between $\$_{r, i}$ and $\$_{e f}$, (9.23) can be simplified, as follows:

$$
\left[\prod \hat{\$}_{r, i}\right]^{T} \$_{e f}=\dot{q}_{3, i}\left[\prod \hat{\$}_{r, i}\right]^{T} \$_{3, i}
$$

where $\Pi=\left[\begin{array}{cc}0_{3} & I_{3} \\ I_{3} & O_{3}\end{array}\right], I_{3}$ is the $3 \times 3$ identity matrix, and $O_{3}$ is the $3 \times 3$ zero matrix. Expanding (9.26) for all the limbs of the mechanism, the velocity equation is found:

$$
\left[\begin{array}{cc}
\left(\boldsymbol{b}_{1} \times \boldsymbol{u}_{3,1}\right)^{T} & \boldsymbol{u}_{3,1}^{T} \\
\left(\boldsymbol{b}_{2} \times \boldsymbol{u}_{3,2}\right)^{T} & \boldsymbol{u}_{3,2}^{T} \\
\vdots & \vdots \\
\left(\boldsymbol{b}_{6} \times \boldsymbol{u}_{3,6}\right)^{T} & \boldsymbol{u}_{3,6}^{T}
\end{array}\right]\left[\begin{array}{c}
\boldsymbol{\omega} \\
\boldsymbol{v}_{o}
\end{array}\right]=I_{6}\left[\begin{array}{c}
\dot{q}_{3,1} \\
\dot{q}_{3,2} \\
\vdots \\
\dot{q}_{3,6}
\end{array}\right]
$$

where the first term on the left of (9.27) accounts for $J_{x}$, and the first term on the right of (9.27) accounts for $J_{q}$, being $I_{6}$ the $6 \times 6$ identity matrix. 


\subsection{Singular Configurations}

On the other hand, a singular configuration of the mechanism occurs when the instantaneous kinematic equation of the mechanism: $J_{x} \dot{\boldsymbol{x}}=J_{q} \dot{\boldsymbol{q}}$ can not be solved since $J_{x}$ and/or $J_{q}$ becomes singular, which implies that the determinant of $J_{x}$ and/or $J_{q}$ goes to zero.

In these particular configurations, the mechanism gains or loses one or more degrees of freedoms instantaneously, and therefore loses its stiffness completely.

The detection of the singular configurations of the $6 \mathrm{UPS}$ is a very complex task. Even though, there are some singular configurations already identified (145), the analytical detection still remains open. Therefore, a more practical approach is the numerical detection of the configurations where the determinant of $J_{x}$ goes zero. $J_{q}$ it is not considered, since for this mechanism $J_{q}=I_{6}$, where $I_{6}$ is the $6 \times 6$ identity matrix, therefore: $\operatorname{det}\left(J_{q}\right)=1$, for all the configurations.

The orientation workspace of the mechanism is reduced by the singular configurations of the mechanism, as it is presented in Fig.9.11.

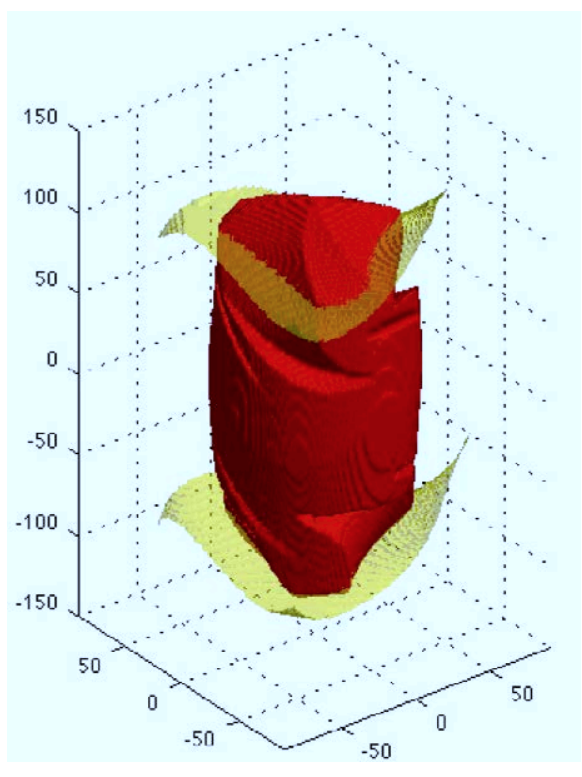

Figure 9.11: 6UPS orientation workspace and singular configurations for $\boldsymbol{r}=[0,0,700]$.

Solid volumen representation for the workspace and singular configurations surface.

This singular surface divides the workspace into three regions: top, middle and bottom. Thus the effective workspace of the mechanism is reduced, to $\pm 90^{\circ}$ for $Y$ aw rotation. 


\section{KINEMATIC ANALYSIS}

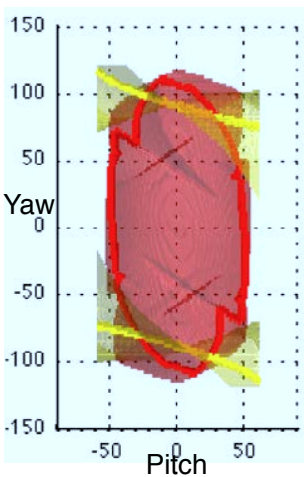

(a)

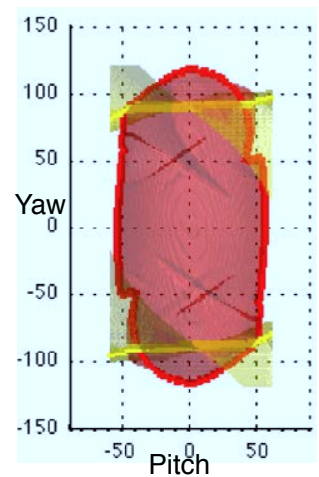

(b)

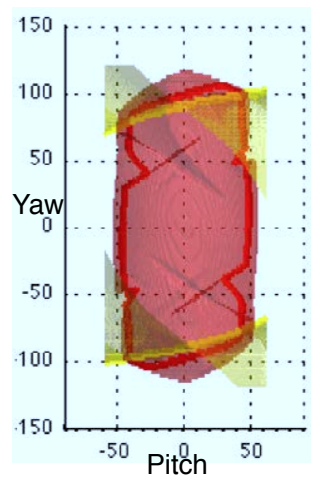

(c)

Figure 9.12: 6 UPS orientation workspace and singular configurations for $\boldsymbol{r}=[0,0,700]$. : slice with plane $R o l l=-30^{\circ}$. : slice with plane $R o l l=0^{\circ}$. : slice with plane $R o l l=30^{\circ}$.

\subsection{Conclusions}

As it is well known, the inverse kinematic problem of the PM is really straight forward. In contrast, its direct kinematic counterpart is not, therefore numerical approaches must be implemented.

In this work, the Newton-Raphson method was implemented with a generic constraint function. Even though, this alternative is currently exploited by many researchers, in this work was performed a complete analysis of its performance in terms of three Key Performance Indexes along the complete workspace of the mechanism considering different initial conditions. The results has proven an $98 \%$ in the rate of convergence of the method implemented and in general the method converges in less that 10 iterations.

Regarding the workspace of the mechanism, it was shown that it is not possible to give global information about it, given that the mechanism has 6DOF. Therefore, it most usual give local information about it, such as maximum possible translation for given orientation and vice-versa. As it was shown, the effective workspace of the mechanism is drastically reduced by presence of singular configurations. Furthermore, in the pictures presented it is observed that the maximum effective workspace is almost $80 \%$ of the total workspace. 


\section{0}

\section{Dynamic Analysis}

"... Mutation is the key to our evolution.

It has enabled to evolve from a single cell into the dominant specie on the planet. ... every few hundred of millenniums evolution leaps forward..."

Charles Xavier

\subsection{Introduction}

The accurate development of a dynamic model play an important role in the field of robotics, since it allows to simulate the robotic system in a computer and design control strategies of higher performance by incorporating more structural information.

In particular, the development of a closed form solution for the dynamical model capable to be implemented in a real time system is of especial interest for control purposes of real mechanism.

The formulation of the dynamic model of PM and the derivation of its dynamic equations is a very complex task since its inherent closed-loop structure provides highly coupled kinematic constraints. 


\subsection{Dynamic Modeling of PMs}

There are several techniques used to solved the dynamic problem of closed-loop systems. One of the most classical method is the definition of a spanning tree of the system where substructures are defined and solved individually, and then loop-closure constraints are applied (53), (98). In particular, in (91), each leg of the PM is a substructure and their dynamics equations are derived using Kane's equation, and then the loop-closure constraints is imposed by implementing Lagrange multipliers. Another approach based on the definition of an equivalent tree structure of the PM is presented in (78), where the dynamic and kinematics models of the legs can be obtained using any classic method.

Traditional approaches derived from Newton-Euler (NE) and Lagrange formulations and their variations are also used (145).

Approaches based on the method of NE permits the modeling of closed-loop mechanisms by deriving explicit equations of motions for all the elements. Even though NE is a powerful method, this formulation results in poor computational efficiency, since all the forces acting on the individual links are computed, including all constraint forces and moments between links, which are useful for design purposes but not for real-time control.

In order to overcame the unnecessary computation of all the forces, in (46) it is presented an strategy for efficient elimination of the constraint reaction forces, resulting in a computationally economical procedure for inverse dynamics and a straightforward derivation of dynamic equations.

Another strategy to improve the efficiency of the NE formulation, is to exploit the inherent parallelism of the computation of the dynamic equation in a parallel computing environment as proposed in (60),(45).

The Lagrange formulation is based on the computation of the energy of the system, it is well structured and can be expressed in closed form. It eliminates all of the unwanted reaction forces and moments at the outset, and thus it is more efficient than the NE method. In contrast, this formulation requires of the definition of a set of independent generalized coordinated, complex symbolic calculation in order to obtain the partial derivatives of the Lagrangian and large amount of numerical computation to evaluated them. In (83) it is presented the dynamics analysis of a 3DoF PM using this approach, defining the dynamics equations in the joint space and determining the forces/torques required to follow a prescribed trajectory. In (11), the dynamic analysis of a $4 \mathrm{DoF}$ PM with parallelogram joints considering all the passive variable is presented. The Lagrangian of the PM is formulated from two independent substructure: limbs 
(whose dynamics are obtained in terms of the actuated and the passive joints), and moving platform (whose dynamics is expressed in terms of the output coordinate). The dynamic model is completed combining these two subsystem, using Jacobian matrices that implicitly contain the kinematic constraints that link them.

There are also some attempts to take advantage of both method by combining them as in (62), where it is used the Lagrange formulation to derive the constraint forces at the joint in the moving platform for each leg, and then incorporate them with N-E to obtain the complete expression of the dynamics of the platform.

The principle of virtual work (VW) is another technique employed for the dynamic modeling of PM. This formulation is also based on the computation of the energy of the system. This principle states that a system is under equilibrium if and only if the virtual work developed by all external, internal and inertial forces vanish for any infinitesimal virtual displacement, who is compatible with the kinematics constraints of the system (145). Since constraint forces and moments do not need to be computed, this approach leads to faster computational algorithms, which is an important advantage for the purposes of control of a manipulator.

One of the first formulation implementing the virtual work was introduced in (147), where the inertial force and moment are computed using the linear an angular accelerations of each body. Then, the PM is considered to be in static equilibrium, therefore the input force are derived by means of the virtual work concept. In (146), is introduced the concept of the link Jacobian matrices, presenting a methodology for the formulation of the inverse dynamic of PM. This work is further extended and completed in (153), formulating the direct and inverse dynamic model of the general 6UPS as the general form used in serial manipulators. The principle of virtual work is also implemented in (1) for solving the direct dynamics of a 3RRP spherical PM, which also includes an algorithm for the selection of the admissible solution of the direct kinematics and the definition of the direct link Jacobian matrices .

Another approach based on the principle of the virtual work that make use of the screw theory for its formulation is presented in (55).

\subsection{Dynamic Model Based on Virtual Work Principle}

The principle of virtual work for a parallel manipulator can be stated as (145):

$$
\delta \boldsymbol{q}^{T} \boldsymbol{\tau}+\delta \boldsymbol{x}_{p}^{T} \boldsymbol{F}_{p}+\sum \delta \boldsymbol{x}_{i}^{T} \boldsymbol{F}_{i}
$$

On the other hand, the virtual displacement of the moving platform must be compatible with the kinematic constraints imposed by the joints, i.e. there exist a relation- 
ship between $\delta \boldsymbol{x}_{p}$ and $\delta \boldsymbol{q}$ and $\delta \boldsymbol{x}_{i}$. This relationship is given by the respective Jacobian matrices:

$$
\begin{gathered}
\delta \boldsymbol{q}=\boldsymbol{J}_{p} \delta \boldsymbol{x}_{p}, \\
\delta \boldsymbol{x}_{i},=\boldsymbol{J}_{i} \delta \boldsymbol{x}_{p},
\end{gathered}
$$

Thus, (10.1) can be reformulated as follows:

$$
\delta \boldsymbol{q}^{T}\left(\boldsymbol{J}_{p} \boldsymbol{\tau}+\boldsymbol{F}_{p}+\sum \boldsymbol{J}_{i} \boldsymbol{F}_{i}\right)=0 .
$$

Since (10.5), is valid for any virtual displacement $\delta \boldsymbol{x}_{p}$, it can be expressed:

$$
\boldsymbol{J}_{p} \boldsymbol{\tau}+\boldsymbol{F}_{p}+\sum \boldsymbol{J}_{i} \boldsymbol{F}_{i}=0
$$

This last expression describes the dynamics of a parallel manipulator. However, it doesn't resembles at all with the general form presented in (2.9).

In order to do so, all the forces of the model must be expressed as follows:

$$
\boldsymbol{F}_{j}=\tau_{e x t, j}-\boldsymbol{M}_{j} \ddot{\boldsymbol{x}}_{j}-\boldsymbol{C}_{j} \dot{\boldsymbol{x}}_{j}-\boldsymbol{g}_{j}
$$

and reformulate the model:

$$
\boldsymbol{J}_{p} \boldsymbol{\tau}+\left(\tau_{e x t, p}-\boldsymbol{M}_{p} \ddot{\boldsymbol{x}}_{p}-\boldsymbol{C}_{p} \dot{\boldsymbol{x}}_{p}-\boldsymbol{g}_{p}\right)+\sum \boldsymbol{J}_{i}\left(\tau_{e x t, i}-\boldsymbol{M}_{i} \ddot{\boldsymbol{x}}_{i}-\boldsymbol{C}_{i} \dot{\boldsymbol{x}}_{i}-\boldsymbol{g}_{i}\right)=0 .
$$

Considering the following velocities and accelerations relations:

$$
\begin{aligned}
\dot{\boldsymbol{x}}_{i} & =\boldsymbol{J}_{i} \dot{\boldsymbol{x}}_{p} \\
\ddot{\boldsymbol{x}}_{i} & =\dot{\boldsymbol{J}}_{i} \dot{\boldsymbol{x}}_{p}+\boldsymbol{J}_{i} \ddot{\boldsymbol{x}}_{p}
\end{aligned}
$$

Rearranging the elements as the following matrices, the virtual work approach can be stated as the general form of the dynamic model (? ):

$$
\begin{aligned}
\boldsymbol{M}_{t} & =\boldsymbol{M}_{p}+\sum_{i} \boldsymbol{J}_{j, i}^{T} \boldsymbol{M}_{j, i} \boldsymbol{J}_{j, i} \\
\boldsymbol{C}_{t} & =\boldsymbol{C}_{p}+\sum_{i}\left(\boldsymbol{J}_{j, i}^{T} \boldsymbol{C}_{j, i} \boldsymbol{J}_{j, i}+\boldsymbol{J}_{j, i}^{T} \boldsymbol{M}_{j, i} \dot{\boldsymbol{J}}_{j, i}\right) \\
\boldsymbol{g}_{t} & =m_{p}\left[\begin{array}{l}
\boldsymbol{g} \\
\mathbf{0}
\end{array}\right]+\sum_{i} m_{j, i} \boldsymbol{J}_{j, i}^{T}\left[\begin{array}{l}
\boldsymbol{g} \\
\mathbf{0}
\end{array}\right]
\end{aligned}
$$




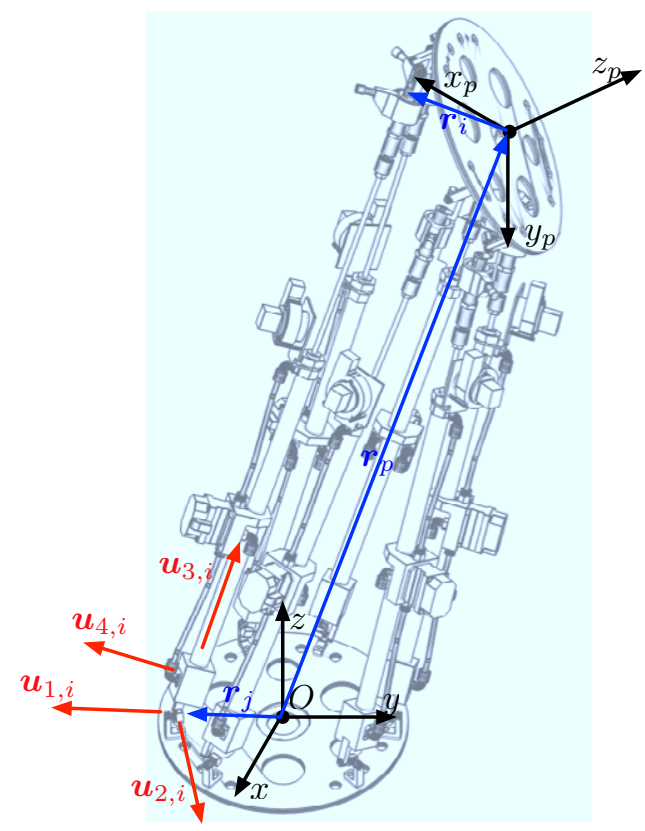

Figure 10.1: Schematic diagram of 6UPSS.

As it can be appreciated in (10.10), the matrices depends of the calculation of the robot's Jacobian matrix $\boldsymbol{J}_{p}$, the Jacobian matrix of each limb $\boldsymbol{J}_{j, i}$ and its time derivative $\dot{\boldsymbol{J}}_{j, i}$.

Therefore, let consider the schematic diagram of the mechanism presented in Fig. 10.1, and lets find the position and velocities of the elements of the system.

The state of the end effector is given by its position $\left[\begin{array}{ll}\boldsymbol{x}_{p} & \boldsymbol{q}_{p}\end{array}\right]$, its velocity $\left[\begin{array}{ll}\boldsymbol{v}_{p} & \boldsymbol{w}_{p}\end{array}\right]$ and its acceleration $\left[\begin{array}{ll}\dot{\boldsymbol{v}}_{p} & \dot{\boldsymbol{w}}_{p}\end{array}\right]$

The first thing is to define the position of the center of mass of each link in terms of the state of the end effector.

The position of the center of gravity of each leg can be defined as

$$
\begin{aligned}
& \boldsymbol{c}_{1, i}={ }^{O} \boldsymbol{R}_{L, i} \boldsymbol{c}_{10, i}, \\
& \boldsymbol{c}_{2, i}=\boldsymbol{l}_{i}-{ }^{O} \boldsymbol{R}_{L, i} \boldsymbol{c}_{20, i}
\end{aligned}
$$

and the rotation matrix ${ }^{O} \boldsymbol{R}_{L i}=\left[\begin{array}{lll}\hat{\boldsymbol{u}}_{4, i}, & \hat{\boldsymbol{u}}_{2, i}, & \hat{\boldsymbol{u}}_{3, i}\end{array}\right]$, is obtained from the elements of 
the axes of the first three joints of each limb as follows:

$$
\begin{aligned}
\hat{\boldsymbol{u}}_{3, i} & =\frac{\boldsymbol{u}_{3, i}}{\left\|\boldsymbol{u}_{3, i}\right\|}, \\
\hat{\boldsymbol{u}}_{2, i} & =\hat{\boldsymbol{u}}_{3, i} \times \hat{\boldsymbol{u}}_{1, i}, \\
\hat{\boldsymbol{u}}_{4, i} & =\hat{\boldsymbol{u}}_{2, i} \times \hat{\boldsymbol{u}}_{3, i},
\end{aligned}
$$

The velocity at the anchoring point of each leg on the moving platform can be expressed in terms of the velocity of the end effector, as follows:

$$
\begin{aligned}
\boldsymbol{v}_{B_{i}} & =\boldsymbol{v}_{p}+w_{p} \times \boldsymbol{b}_{i}, \\
& =\boldsymbol{v}_{p}-\tilde{\boldsymbol{b}}_{i} w_{p},
\end{aligned}
$$

or in terms of the velocities of the joints of each leg as follows:

$$
\begin{aligned}
\boldsymbol{v}_{B_{i}} & =\dot{\theta}_{1, i} \hat{\boldsymbol{u}}_{1, i} \times \boldsymbol{l}_{i}+\dot{\theta}_{2, i} \hat{\boldsymbol{u}}_{2, i} \times \boldsymbol{l}_{i}+\dot{\theta}_{3, i} \hat{\boldsymbol{u}}_{3, i}, \\
& =-\dot{\theta}_{1, i} \tilde{\boldsymbol{l}}_{i} \hat{\boldsymbol{u}}_{1, i}-\dot{\theta}_{2, i} \tilde{\boldsymbol{l}}_{i} \hat{\boldsymbol{u}}_{2, i}+\dot{\theta}_{3, i} \hat{\boldsymbol{u}}_{3, i}
\end{aligned}
$$

from (10.20) it can be obtained useful expression for $\dot{\theta}_{1, i}, \theta_{2, i} \dot{\theta}_{3, i}$ by premultiplying it by $\hat{\boldsymbol{u}_{2, i}} \hat{\boldsymbol{u}_{1, i}} \hat{\boldsymbol{u}_{3, i}}$, respectively as follows,

$$
\begin{aligned}
& \hat{\boldsymbol{u}}_{2, i}^{T} \boldsymbol{v}_{B_{i}}=-\dot{\theta}_{1, i} \hat{\boldsymbol{u}}_{2, i}^{T} \tilde{\boldsymbol{l}}_{i} \hat{\boldsymbol{u}}_{1, i}, \\
& \hat{\boldsymbol{u}}_{1, i}^{T} \boldsymbol{v}_{B_{i}}=-\dot{\theta}_{2, i} \hat{\boldsymbol{u}}_{1, i}^{T} \tilde{\boldsymbol{l}}_{i} \hat{\boldsymbol{u}}_{2, i}+\hat{\boldsymbol{u}}_{1, i}^{T} \hat{\boldsymbol{u}}_{3, i} \dot{\theta}_{3, i}, \\
& \hat{\boldsymbol{u}}_{3, i}^{T} \boldsymbol{v}_{B_{i}}=\dot{\theta}_{3, i},
\end{aligned}
$$

and equating for $\theta_{j, i}$ :

$$
\begin{aligned}
& \theta_{1, i}=\frac{1}{f_{i}} \hat{\boldsymbol{u}}_{2, i}^{T} \boldsymbol{v}_{B_{i}}, \\
& \theta_{2, i}=\frac{1}{f_{i}} \hat{\boldsymbol{u}}_{1, i}^{T}\left(\hat{\boldsymbol{u}}_{3, i} \hat{\boldsymbol{u}}_{3, i}^{T}-\boldsymbol{I}_{3}\right) \boldsymbol{v}_{B_{i}},
\end{aligned}
$$

where $f_{i}=\hat{\boldsymbol{u}}_{1, i}^{T} \tilde{\boldsymbol{l}}_{i} \hat{\boldsymbol{u}}_{2, i}$.

Equating (10.23) and (10.20) the velocity of the actuated joint can be expressed in terms of the the velocity of the end effector, hence, the Jacobian matrix can be derived:

$$
\begin{aligned}
& \dot{\theta}_{3, i}=\hat{\boldsymbol{u}}_{3, i}^{T}\left(\boldsymbol{v}_{p}-\tilde{\boldsymbol{b}}_{i} \boldsymbol{w}_{p}\right), \\
& =\left[\begin{array}{ll}
\hat{\boldsymbol{u}}_{3, i}^{T} & -\hat{\boldsymbol{u}}_{3, i}^{T} \tilde{\boldsymbol{b}}_{i}
\end{array}\right]\left[\begin{array}{c}
\boldsymbol{v}_{p} \\
\boldsymbol{w}_{p}
\end{array}\right] \\
& =\boldsymbol{J}_{p, i}\left[\begin{array}{c}
\boldsymbol{v}_{p} \\
\boldsymbol{w}_{p}
\end{array}\right] \text {. }
\end{aligned}
$$


The velocity at each center of gravity is given by:

$$
\begin{aligned}
\dot{\boldsymbol{c}}_{1, i} & ={ }^{O} \dot{\boldsymbol{R}}_{L, i} \boldsymbol{c}_{10, i}, \\
& =\tilde{\boldsymbol{w}}_{1}{ }^{O} \boldsymbol{R}_{L, i} \boldsymbol{c}_{10, i}, \\
& =\left(\dot{\theta}_{1, i} \tilde{\hat{\boldsymbol{u}}}_{1, i}+\dot{\theta}_{2, i} \tilde{\hat{\boldsymbol{u}}}_{2, i}\right) \boldsymbol{c}_{1, i}, \\
& =-\tilde{\boldsymbol{c}}_{1, i} \hat{\boldsymbol{u}}_{1, i} \dot{\theta}_{1, i}-\tilde{\boldsymbol{c}}_{1, i} \hat{\boldsymbol{u}}_{2, i} \dot{\theta}_{2, i}, \\
& =-\tilde{\boldsymbol{c}}_{1, i} \frac{1}{f_{i}}\left(\hat{\boldsymbol{u}}_{1, i}^{T} \hat{\boldsymbol{u}}_{2, i}+\hat{\boldsymbol{u}}_{2, i} \hat{\boldsymbol{u}}_{1, i}^{T} \hat{\boldsymbol{u}}_{3, i} \hat{\boldsymbol{u}}_{3, i}^{T}-\hat{\boldsymbol{u}}_{2, i} \hat{\boldsymbol{u}}_{1, i}^{T}\right) \boldsymbol{v}_{B_{i}}
\end{aligned}
$$

$$
\begin{aligned}
\dot{\boldsymbol{c}}_{2, i} & =\dot{\boldsymbol{l}}_{i}+{ }^{O} \dot{\boldsymbol{R}}_{L, i} \boldsymbol{c}_{20, i}, \\
& =-\dot{\theta}_{1, i} \tilde{\boldsymbol{u}}_{i} \hat{\boldsymbol{u}}_{1, i}-\dot{\theta}_{2, i} \tilde{\boldsymbol{l}}_{i} \hat{\boldsymbol{u}}_{2, i}+\dot{\theta}_{3, i} \hat{\boldsymbol{u}}_{3, i}+\left(\dot{\theta}_{1, i} \tilde{\boldsymbol{u}}_{1, i}+\dot{\theta}_{2, i} \tilde{\boldsymbol{\boldsymbol { u }}}_{2, i}\right) \boldsymbol{c}_{2, i} \\
& =-\left(\tilde{\boldsymbol{l}}_{i}+\tilde{\boldsymbol{c}}_{2, i}\right)\left(\hat{\boldsymbol{u}}_{1, i} \dot{\theta}_{1, i}+\hat{\boldsymbol{u}}_{2, i} \dot{\theta}_{2, i}\right)+\hat{\boldsymbol{u}}_{3, i} \dot{\theta}_{3, i}, \\
& =-\left(\tilde{\boldsymbol{l}}_{i}+\tilde{\boldsymbol{c}}_{2, i}\right) \frac{1}{f_{i}}\left(\hat{\boldsymbol{u}}_{1, i}^{T} \hat{\boldsymbol{u}}_{2, i}+\hat{\boldsymbol{u}}_{2, i} \hat{\boldsymbol{u}}_{1, i}^{T} \hat{\boldsymbol{u}}_{3, i} \hat{\boldsymbol{u}}_{3, i}^{T}-\hat{\boldsymbol{u}}_{2, i} \hat{\boldsymbol{u}}_{1, i}^{T}\right) \boldsymbol{v}_{B_{i}}+\hat{\boldsymbol{u}}_{3, i} \hat{\boldsymbol{u}}_{3, i}^{T} \boldsymbol{v}_{B_{i}}, \\
& =-\left(\left(\tilde{\boldsymbol{l}}_{i}+\tilde{\boldsymbol{c}}_{2, i}\right) \frac{1}{f_{i}}\left(\hat{\boldsymbol{u}}_{1, i}^{T} \hat{\boldsymbol{u}}_{2, i}+\hat{\boldsymbol{u}}_{2, i} \hat{\boldsymbol{u}}_{1, i}^{T} \hat{\boldsymbol{u}}_{3, i} \hat{\boldsymbol{u}}_{3, i}^{T}-\hat{\boldsymbol{u}}_{2, i} \hat{\boldsymbol{u}}_{1, i}^{T}\right)+\hat{\boldsymbol{u}}_{3, i} \hat{\boldsymbol{u}}_{3, i}^{T}\right) \boldsymbol{v}_{B_{i}}
\end{aligned}
$$

Defining the following matrices,

$$
\begin{aligned}
\boldsymbol{H}_{i} & =\hat{\boldsymbol{u}}_{2, i} \hat{\boldsymbol{u}}_{1, i}^{T} \hat{\boldsymbol{u}}_{3, i} \hat{\boldsymbol{u}}_{3, i}^{T}, \\
\boldsymbol{G}_{i} & =\hat{\boldsymbol{u}}_{1, i} \hat{\boldsymbol{u}}_{2, i}^{T}, \\
\boldsymbol{K}_{1, i} & =\frac{1}{f_{i}}\left(\boldsymbol{G}_{i}-\boldsymbol{G}_{i}^{T}+\boldsymbol{H}_{i}\right), \\
\boldsymbol{K}_{2, i} & =\left(\tilde{\boldsymbol{l}}_{i}+\tilde{\boldsymbol{c}}_{2, i}\right) \boldsymbol{K}_{1, i}+\hat{\boldsymbol{u}}_{3, i} \hat{\boldsymbol{u}}_{3, i}^{T}
\end{aligned}
$$

the Jacobian matrices for each leg can be defined as,

$$
\begin{aligned}
\boldsymbol{J}_{1, i} & =\left[\begin{array}{cc}
-\tilde{\boldsymbol{c}}_{1, i} \boldsymbol{K}_{i} & -\tilde{\boldsymbol{c}}_{1, i} \boldsymbol{K}_{i} \tilde{\boldsymbol{b}}_{i} \\
\boldsymbol{K}_{i} & -\boldsymbol{K}_{i} \tilde{\boldsymbol{b}}_{i}
\end{array}\right], \\
\boldsymbol{J}_{2, i} & =\left[\begin{array}{cc}
-\boldsymbol{K}_{2, i} & \boldsymbol{K}_{2, i} \tilde{\boldsymbol{b}}_{i} \\
\boldsymbol{K}_{1, i} & -\boldsymbol{K}_{1, i} \tilde{\boldsymbol{b}}_{i}
\end{array}\right]
\end{aligned}
$$

The derivatives of the Jacobian matrices will be given by,

$$
\begin{aligned}
\dot{\boldsymbol{J}}_{1, i} & =\left[\begin{array}{cc}
-\dot{\tilde{\boldsymbol{c}}}_{1, i} \boldsymbol{K}_{1, i}-\tilde{\boldsymbol{c}}_{1, i} \dot{\boldsymbol{K}}_{1, i} & -\dot{\tilde{\boldsymbol{c}}}_{1, i} \boldsymbol{K}_{1, i} \tilde{\boldsymbol{b}}_{1, i}-\tilde{\boldsymbol{c}}_{1, i} \dot{\boldsymbol{K}}_{1, i} \tilde{\boldsymbol{b}}_{i}-\tilde{\boldsymbol{c}}_{1, i} \boldsymbol{K}_{1, i} \dot{\tilde{\boldsymbol{b}}}_{i} \\
\dot{\boldsymbol{K}}_{1, i} & -\dot{\boldsymbol{K}}_{1, i} \tilde{\boldsymbol{b}}_{i}-\boldsymbol{K}_{1, i} \dot{\tilde{\boldsymbol{b}}}_{i}
\end{array}\right], \\
\dot{\boldsymbol{J}}_{2, i} & =\left[\begin{array}{cc}
\dot{\boldsymbol{K}}_{2, i} & \dot{\boldsymbol{K}}_{2, i} \tilde{\boldsymbol{b}}_{1, i}+\boldsymbol{K}_{2, i} \dot{\tilde{\boldsymbol{b}}}_{i} \\
\dot{\boldsymbol{K}}_{1, i} & -\dot{\boldsymbol{K}}_{1, i} \tilde{\boldsymbol{b}}_{i}-\boldsymbol{K}_{1, i} \dot{\tilde{\boldsymbol{b}}}_{i}
\end{array}\right],
\end{aligned}
$$


where,

$$
\begin{aligned}
\dot{\boldsymbol{K}}_{1, i} & =\frac{1}{f_{i}}\left(\dot{\boldsymbol{G}}_{i}-\dot{\boldsymbol{G}}_{i}^{T}+\dot{\boldsymbol{H}}_{i}\right)-\frac{\dot{f}_{i}}{f_{i}^{2}}\left(\boldsymbol{G}_{i}-\boldsymbol{G}_{i}^{T}+\boldsymbol{H}_{i}\right) \\
\dot{\boldsymbol{K}}_{2, i} & =\tilde{\boldsymbol{c}}_{2, i} \boldsymbol{K}_{1, i}+\boldsymbol{c}_{2, i} \dot{\boldsymbol{K}}_{1, i}-\dot{\boldsymbol{u}}_{3, i} \boldsymbol{u}_{3, i}^{T}-\boldsymbol{u}_{3, i} \dot{\boldsymbol{u}}_{3, i}^{T} \\
\dot{f}_{i} & \left.=\boldsymbol{u}_{1, i}^{T} \tilde{\boldsymbol{l}}_{i} \dot{\boldsymbol{u}}_{2, i}-\boldsymbol{u}_{1, i}^{T} \tilde{\boldsymbol{u}}_{2, i}\right) \dot{\boldsymbol{d}}_{i} \\
\dot{\boldsymbol{G}}_{i} & =\dot{\boldsymbol{u}}_{1, i} \boldsymbol{u}_{2, i}^{T}+\boldsymbol{u}_{1, i} \dot{\boldsymbol{u}}_{2, i}^{T} \\
\dot{\boldsymbol{H}}_{i} & =\dot{\boldsymbol{u}}_{2, i} \boldsymbol{u}_{1, i}^{T} \boldsymbol{u}_{3, i} \boldsymbol{u}_{3, i}^{T}+\boldsymbol{u}_{2, i} \boldsymbol{u}_{1, i}^{T} \dot{\boldsymbol{u}}_{3, i} \boldsymbol{u}_{3, i}^{T}+\boldsymbol{u}_{2, i} \boldsymbol{u}_{1, i}^{T} \boldsymbol{u}_{3, i} \dot{\boldsymbol{u}}_{3, i}^{T}
\end{aligned}
$$

\subsection{Model Validation}

The accuracy of the dynamic model presented in the previous section is analyzed by comparing its results with a simulated model in ADAMS imported from the CAD design with its real physical properties.

The forces exerted by the prismatic actuator are obtained for several trajectories. These trajectories are sinusoidal type and defined in the task space. Since input of the model implemented in ADAMS are the states of the prismatic actuator, the trajectories are converted in the joint space by means of the inverse kinematics.

The forces obtained from the simulation are analyzed and discussed. The accuracy of the model is quantified in terms of the maximum absolute and relative difference.

An schematic diagram of the methodology used for the validation is presented in Fig.10.2.

The physical properties of the PM used in the inverse dynamic model are taken from the CAD design in Inventor@, and are presented in Table 10.1.

\subsubsection{Simulations}

The trajectories are defined in the task space by the coordinate vectors:

- $\boldsymbol{q}(t)=[\boldsymbol{r}(t), \boldsymbol{p}(t)]$, where $\boldsymbol{r}(t)=[x, y, z]$ is the position of the center of mass of the end effector (i.e. the moving platform), and $\boldsymbol{p}(t)=\left[e_{0}, e_{1}, e_{2}, e_{3}\right]$ is its orientation.

- $\dot{\boldsymbol{q}}(t)=\left[\dot{\boldsymbol{r}}(t), \boldsymbol{w}_{p}(t)\right]$, where $\dot{\boldsymbol{r}}(t)=\left[v_{x}, v_{y}, v_{z}\right]$ is the linear velocity and $\boldsymbol{w}_{p}=$ $\left[w_{x}, w_{y}, w_{z}\right]$ is the angular velocity.

- $\ddot{\boldsymbol{q}}=\left[\ddot{\boldsymbol{r}}, \dot{\boldsymbol{w}}_{p}\right]$, where $\ddot{\boldsymbol{r}}=\left[a_{x}, a_{y}, a_{z}\right]$ is the linear acceleration and $\dot{\boldsymbol{w}}_{p}=\left[n_{x}, n_{y}, n_{z}\right]$ is the angular acceleration. 


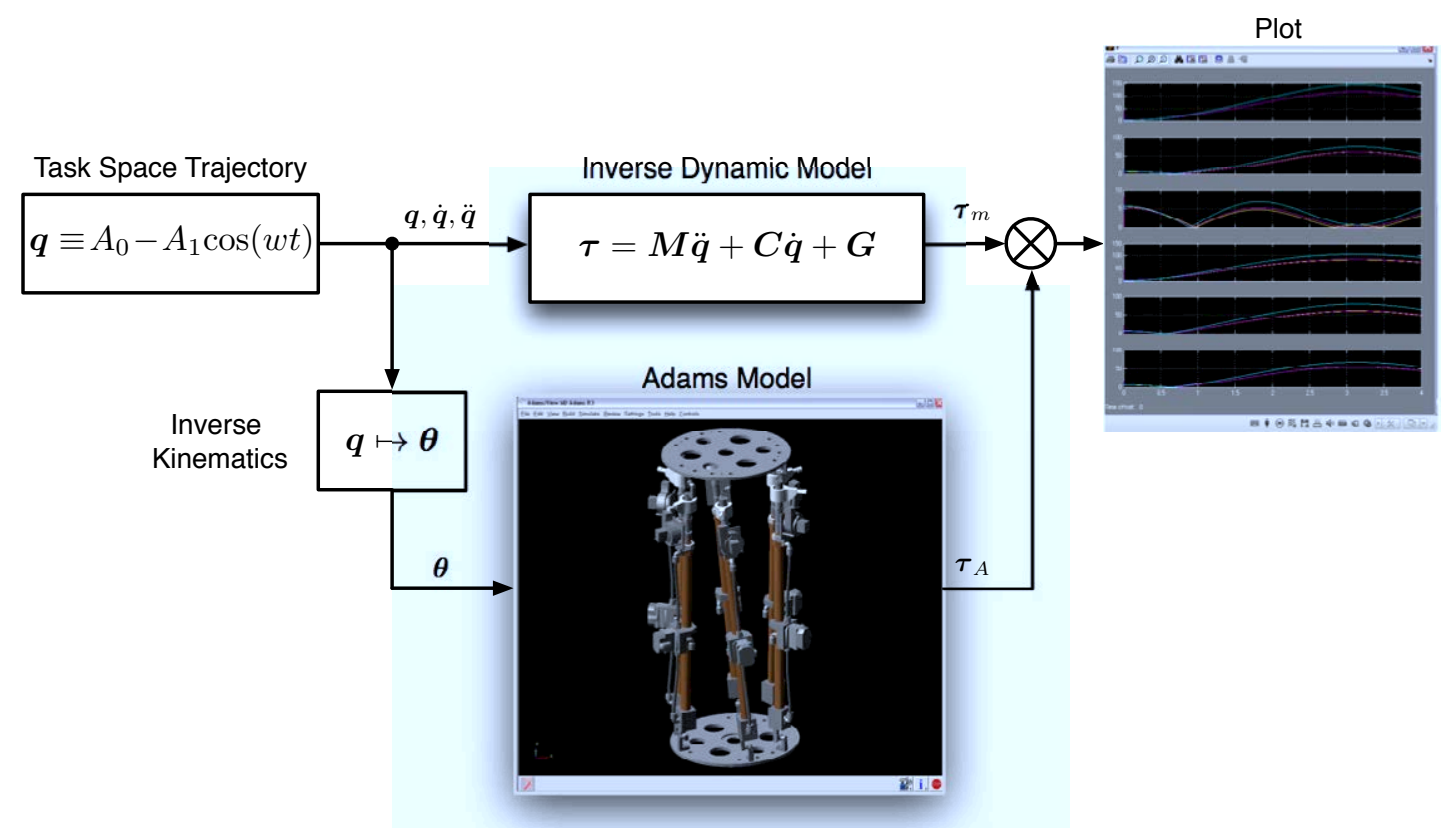

Figure 10.2: Schematic diagram for the dynamic model validation.

Table 10.1: Dynamic properties of the PM

\begin{tabular}{|c|c|c|c|c|c|}
\hline \multirow[t]{2}{*}{ Element } & \multirow[t]{2}{*}{$\begin{array}{l}\operatorname{mass} \\
{[K g]}\end{array}$} & $\begin{array}{c}\text { Center of Gravity } \\
\qquad[\mathrm{mm}]\end{array}$ & \multicolumn{3}{|c|}{$\begin{array}{c}\text { Inertia Matrix } \\
{\left[\mathrm{Kg} \mathrm{mm}^{2}\right]}\end{array}$} \\
\hline & & & $\lceil 7016$ & 0 & 0 \\
\hline \multirow[t]{3}{*}{ Mov } & 1.78 & {$[0,0,34.2]^{\star}$} & 0 & 7016 & 0 \\
\hline & & & 0 & 0 & $14002]$ \\
\hline & & & $\lceil 15637$ & -0.4 & -42.9 \\
\hline \multirow[t]{3}{*}{$L 1_{i}$} & 1.108 & {$[0,0,226]^{*}$} & -0.4 & 15231 & -614 \\
\hline & & & -42 & -614 & 946 \\
\hline & & & 3151 & 0 & $-16.9]$ \\
\hline \multirow[t]{2}{*}{$L 2_{i}$} & 0.193 & {$[0,0,-152]^{\dagger}$} & 0 & 3156 & -7.9 \\
\hline & & & -16.9 & -7.9 & 11.5 \\
\hline
\end{tabular}

* referenced from the center of the circle defined by the center of the spherical.

* referenced from the center of the $i t h$ universal joint.

$\dagger$ referenced from the center of the ith spherical joint. 
The desired coordinate vector are defined implementing a sinusoidal type planing for position and orientation separately, and then performing the corresponding time derivative. Therefore, the desired position can be defined as follows,

$$
\begin{aligned}
\boldsymbol{r} & =\boldsymbol{a}_{0}-a_{1} \cos (w t) \boldsymbol{u}_{r}, \\
\dot{\boldsymbol{r}} & =a_{1} w \sin (w t) \boldsymbol{u}_{r}, \\
\ddot{\boldsymbol{r}} & =a_{1} w^{2} \cos (w t) \boldsymbol{u}_{r},
\end{aligned}
$$

where $\boldsymbol{u}_{r}$ is the direction of the translation, and the constants $\boldsymbol{a}_{0}$ and $a_{1}$ define the initial position and maximum displacement, respectively. $w$ is the frequency of the displacement.

Since for the simulations it is only considered pure rotations, then the orientation planing can be defined as follows:

$$
\begin{aligned}
\theta & =\theta_{0}(1-\cos (w t)), \\
\boldsymbol{p} & =\left[\cos (\theta / 2), \sin (\theta / 2) \boldsymbol{u}_{p}\right], \\
\boldsymbol{w} & =\theta_{0} w \sin (w t) \boldsymbol{u}_{p}, \\
\dot{\boldsymbol{w}} & =\theta_{0} w^{2} \cos (w t) \boldsymbol{u}_{p},
\end{aligned}
$$

where $\theta_{0}$ is the maximum rotation along the axis of rotation $\boldsymbol{u}_{p}$.

For all the trajectories it was defined a frequency of $w=1[\mathrm{rad} / \mathrm{s}]$, and it was considered a simulation time of $4[s]$. The parameters for all the trajectories are presented in Tab.10.2.

During the simulations it is obtained the maximum absolute error $\left(\left|\tilde{F}_{\text {Max }}\right|\right)$ and the maximum relative error $\left(\tilde{F}_{M a x} / F_{A}\right)$. The curves obtained during simulation are presented in the appendix.

Table 10.2: Simulation Results

\begin{tabular}{cccccccc}
\hline Trajectory & $\boldsymbol{a}_{0}$ & $a_{1}$ & $\boldsymbol{u}_{r}$ & $\theta_{0}$ & $\boldsymbol{u}_{p}$ & $|\tilde{F}|_{\max }$ & $\tilde{F}_{M a x} / F_{A} \star \%$ \\
\hline Tx & {$[100,0,583.5]$} & 100 & {$[1,0,0]$} & - & - & 0.64 & 5.54 \\
Ty & {$[0,100,583.5]$} & 100 & {$[0,1,0]$} & - & - & 0.97 & 5.11 \\
Tz & {$[0,0,683.5]$} & 100 & {$[0,0,1]$} & - & - & 0.40 & 1.84 \\
Rx & {$[0,0,583.5]$} & - & - & $\pi / 4$ & {$[1,0,0]$} & 0.70 & 10.00 \\
Ry & {$[0,0,583.5]$} & - & - & $\pi / 4$ & {$[0,1,0]$} & 0.82 & 9.39 \\
Rz & {$[0,0,583.5]$} & - & - & $\pi / 4$ & {$[0,0,1]$} & 5.50 & 37.06 \\
\hline
\end{tabular}



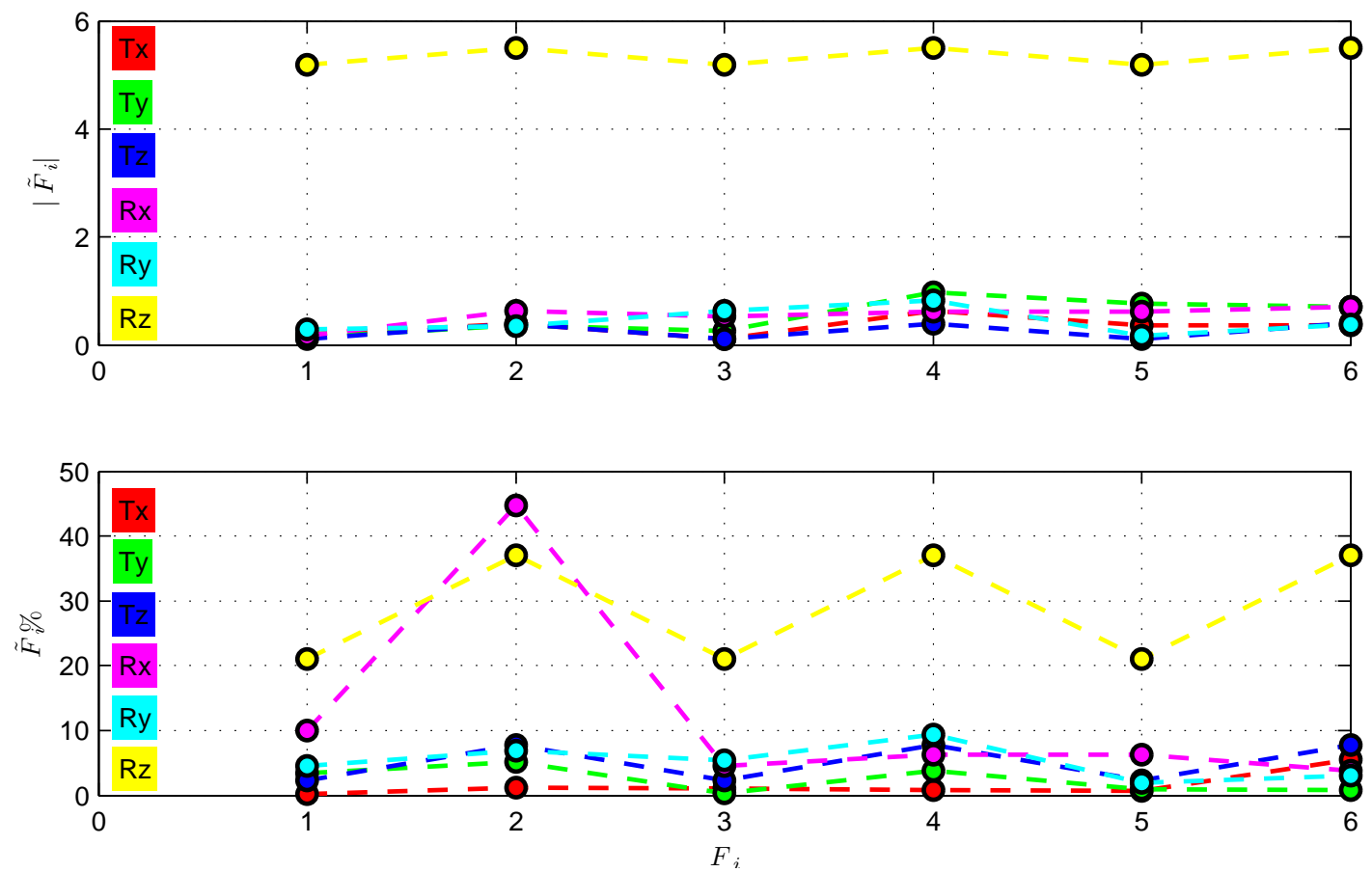

Figure 10.3: Absolute and relative maximum error for all the simulated trajectories.

\subsection{Conclusions}

The model based on the virtual work principle allows a fast computation of the resulting dynamics of the mechanism. The validation process with ADAMS environment have proven to be an efficient and reliable formulation. 
10. DYNAMIC ANALYSIS 


\title{
11
}

\section{Control Strategies for SG}

\author{
"Most men would rather deny a hard truth than face it" \\ Tyrion Lannister
}

\subsection{Introduction}

The objective of the design and development of strategies and algorithms to control a robot is to guarantee that the robot is capable of accomplishing a defined task withstanding external perturbation and uncertainties present in the environment.

Even the more simple strategies requires of path planing, processing information coming from the sensors, implementation of laws and decision making, and of course a basic knowledge of the state of the robot at a given time.

From the conceptual point of view, the control of a robot can be performed in the joint space or in the task space. In general, the latter one is mostly recommended when the robot interacts directly with the environment, although the implementation of such strategies are more complex since it will require of the $6 \mathrm{DOF}$ sensor to measure the displacement of the end effector.

In this chapter, is presented the control strategies implemented for the PM and their performance is evaluated over some movements that tries to emulate the normal pick-and-place task and trajectory following. 


\section{CONTROL STRATEGIES FOR SG}

\subsection{Controllers for Hydraulic Hexapode PM}

The high nonlinear nature of parallel robots discards the implementation of traditional and single PID control since it may only perform well at a particular operating range. Therefore, in order to overcome this limitation, it is preferred the implementation self tuning PID, where the parameters can be manipulated online based on parameter estimation. The criteria and the tools implemented for the self tuning process is very diverse. In (3), it is proposed fuzzy technique to give the logic capability of computing the membership function parameters that effectively allow the associated fuzzy inference system to track the given input and output data and tuning the PID gains of the control system.

In high precision applications, vibration phenomena are always present and are usually undesired. To overcome this issue (4), implements a gain scheduling approach, where the workspace of the PM under study is divided into several working points and a robust $H_{\infty}$ controller is synthesized for each of them. For intermediate points an interpolated model is calculated based on the model of the surrounding working points which are responsible for the determination of the interpolated controller. Small Gain Theorem is used for the determination of the overall robust stability. $H_{\infty}$ is also adopted in (84), to compensate the modeling errors in the inverse dynamic controller, or also called computed-torque controller for position control. Force computation is

needed to reduce tracking error in high speed tracking task. For this purpose, the inverse dynamic is implemented in (42).

(48) introduces a model based feedback controller that consider both dynamic model of the PM and the hydraulic actuator model, in order to find a decoupled invariant error dynamics that could describe the complex relation between the force and current input of the actuator.

In (63) is presented a cascade control based on the sliding mode in the joint space to realize trajectory tracking, where the hydraulics dynamic is separated from the mechanical part. The inner loop is aimed at controlling the actuator load pressure, independent of the resulting motions of load. The outer loop is concerned with the control of the actuator load.

(80) is proposed a robust nonlinear task space control with friction estimator. In order to perform the control in the task space it is implemented a estimation method based on the Newton-Raphson method and alpha -beta tracker algorithm to avoid costly 6 DoF sensor and model based nonlinear observer. 


\subsection{Trajectory Generation}

The movement of the PM is defined in the task space as a timed sequence set of configurations (i.e. position and orientation of the moving platform). This set of configurations is mapped into the joint space implementing the inverse kinematic of the manipulator and the resulting states of the actuator are interpolated using the strategy described below.

The objective of the strategy implemented in this work is to safeguard the continuity on the velocity and acceleration profile in order to generate smooth trajectories. Because of this, it is defined a three regime velocity profile governed by the following set of equations (see Fig. 11.1 for clarification) :

Acceleration regime: $t_{0} \leq t<t_{a}$

$$
\begin{aligned}
& y=a_{0}+a_{1}\left(t-t_{0}\right)-a_{2} \sin \left(w\left(t-t_{0}\right)\right) \\
& \dot{y}=a_{1}-a_{2} w \cos \left(w\left(t-t_{0}\right)\right) \\
& \ddot{y}=a_{2} w^{2} \sin \left(w\left(t-t_{0}\right)\right)
\end{aligned}
$$

Constant velocity regime: $t_{a} \leq t<t_{c v}$

$$
\begin{aligned}
& y=b_{0}+b_{1}\left(t-t_{0}-t_{a}\right) \\
& \dot{y}=b_{1} \\
& \ddot{y}=0
\end{aligned}
$$

Deceleration regime: $t_{c v} \leq t<t_{d}$

$$
\begin{aligned}
& y=c_{0}+c_{1}\left(t-t_{0}-t_{a}-t_{c v}\right)-c_{2} \sin \left(w\left(t-t_{0}-t_{a}-t_{c v}\right)\right) \\
& \dot{y}=c_{1}-c_{2} w \cos \left(w\left(t-t_{0}-t_{a}-t_{c v}\right)\right) \\
& \ddot{y}=c_{2} w^{2} \sin \left(w\left(t-t_{0}-t_{a}-t_{c v}\right)\right)
\end{aligned}
$$

Given the initial conditions $y_{0}$ and $\dot{y}_{0}$ and final conditions $y_{f}$ and $\dot{y}_{f}=0$, the desired duration of the point-to-point movement $t_{m}=t_{a}+t_{c v}+t_{d}$ and considering the boundaries conditions of each regime and a symmetrical velocity profile (i.e $t_{a}=t_{d}$ ); 


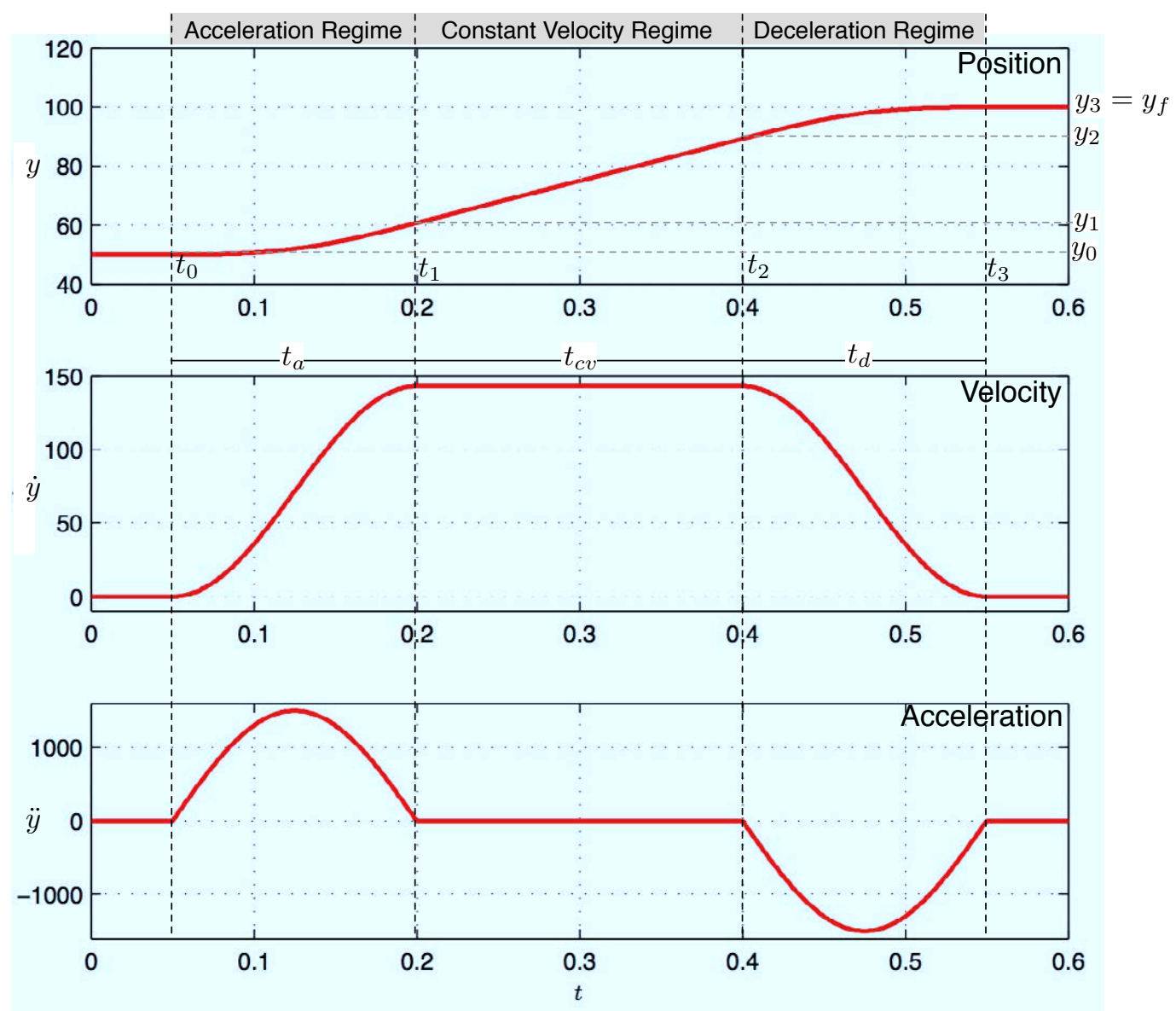

Figure 11.1: Velocity profile for actuators.

the coefficients of (11.1), (11.2) and (11.3) can be found and are given as follows:

$$
\begin{aligned}
t_{c v} & =t_{m}-2 t_{a} & b_{0} & =a_{0}+a_{1} t_{a} \\
w & =\frac{\pi}{t_{a}} & b_{1} & =2 a_{1}-\dot{y}_{0} \\
a_{0} & =y_{0} & c_{0} & =b_{0}+b_{1} t_{c v} \\
a_{1} & =\frac{y_{f}-y_{0}+\dot{y}_{0}\left(t_{c v}+0.5 t_{a}\right)}{2\left(t_{m}-t_{a}\right)} & c_{1} & =0.5 b_{1} \\
a_{2} & =\frac{a_{1}-\dot{y}_{0}}{w} & c_{2} & =\frac{c_{1}}{w}
\end{aligned}
$$

The sinusoidal-linear-sinusoidal (SLS) strategy with zero initial and final velocity is implemented for the point-to-point task movement presented in Fig.11.3(henceforth, movement 1 - diagonal movement). In this movement, the PM is taken from its home 
position to the center point (defined by the intersection of the diagonals of the cube) and then the PM must reach each vertex of the cube returning to the center point (see the black circles in left figure), without modifying its orientation.

The red curves, in the 3D plot, are the expected resulting movement of the PM using the SLS planner in the joint space. Given that the trajectory is generated in the joint space it can be seen, as it is expected, that the resulting movement of the PM does not follow a strait line (see the dashed-black line).

At the right of Fig.11.3 is presented the time evolution of the $x, y$ and $z$ coordinates of the center of mass of the moving platform (red, green and blue curves respectively).
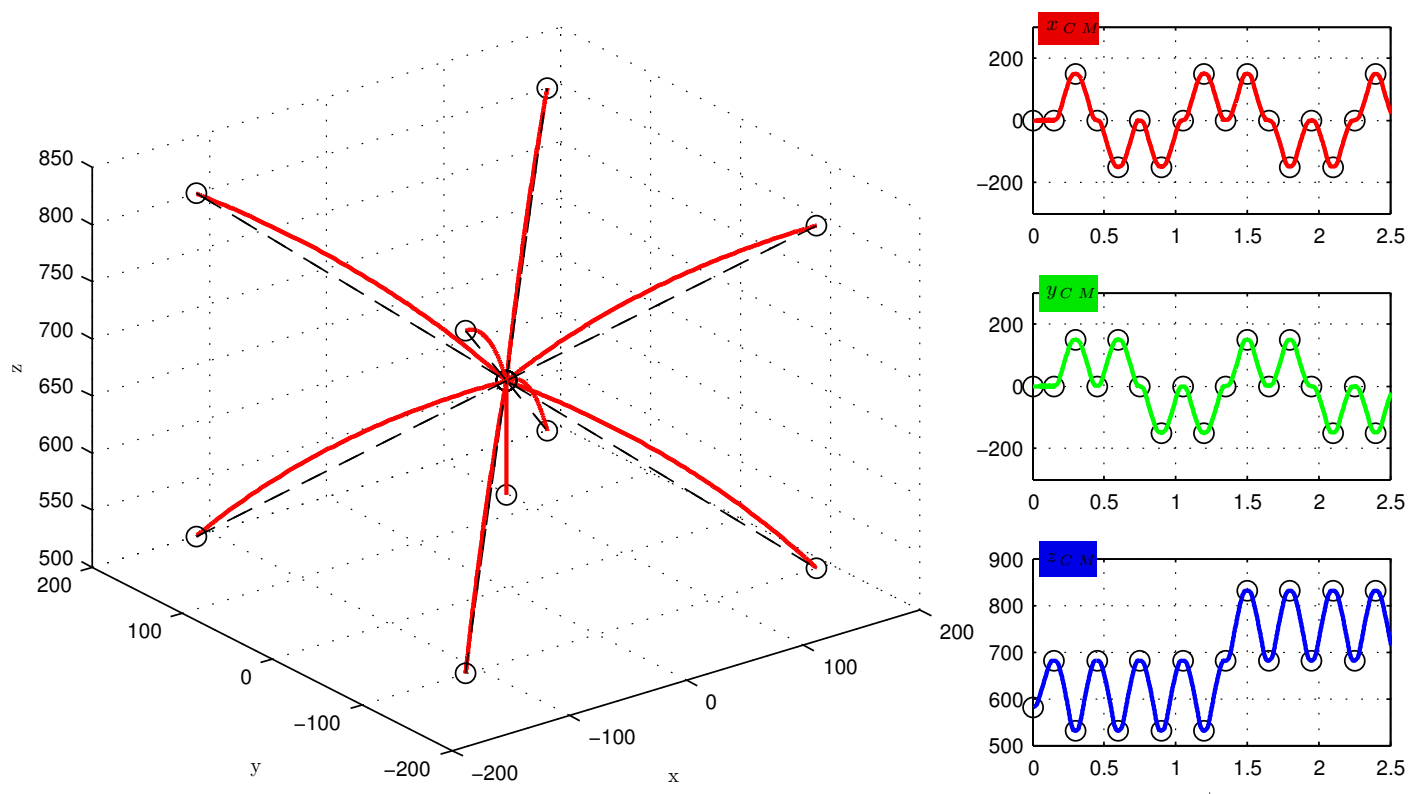

Figure 11.2: Movement 1: Point to point movement.

Considering a trajectory defined by a set of configurations in the task space, the SLS strategy can be easily adapted for the interpolation of several points. As a manner of fact, this adaptation consist on removing the deceleration regime from the interpolation but for the last point of the trajectory. This approach was implemented for the generation of the circular movement presented in Fig.11.3. In more detail, the movement is composed of two part, the first one takes the end effector of the PM from home to the beginning of the circular trajectory; and the second, is the circular trajectory in plane $z=631$.Both part begins and end with zero velocity. 

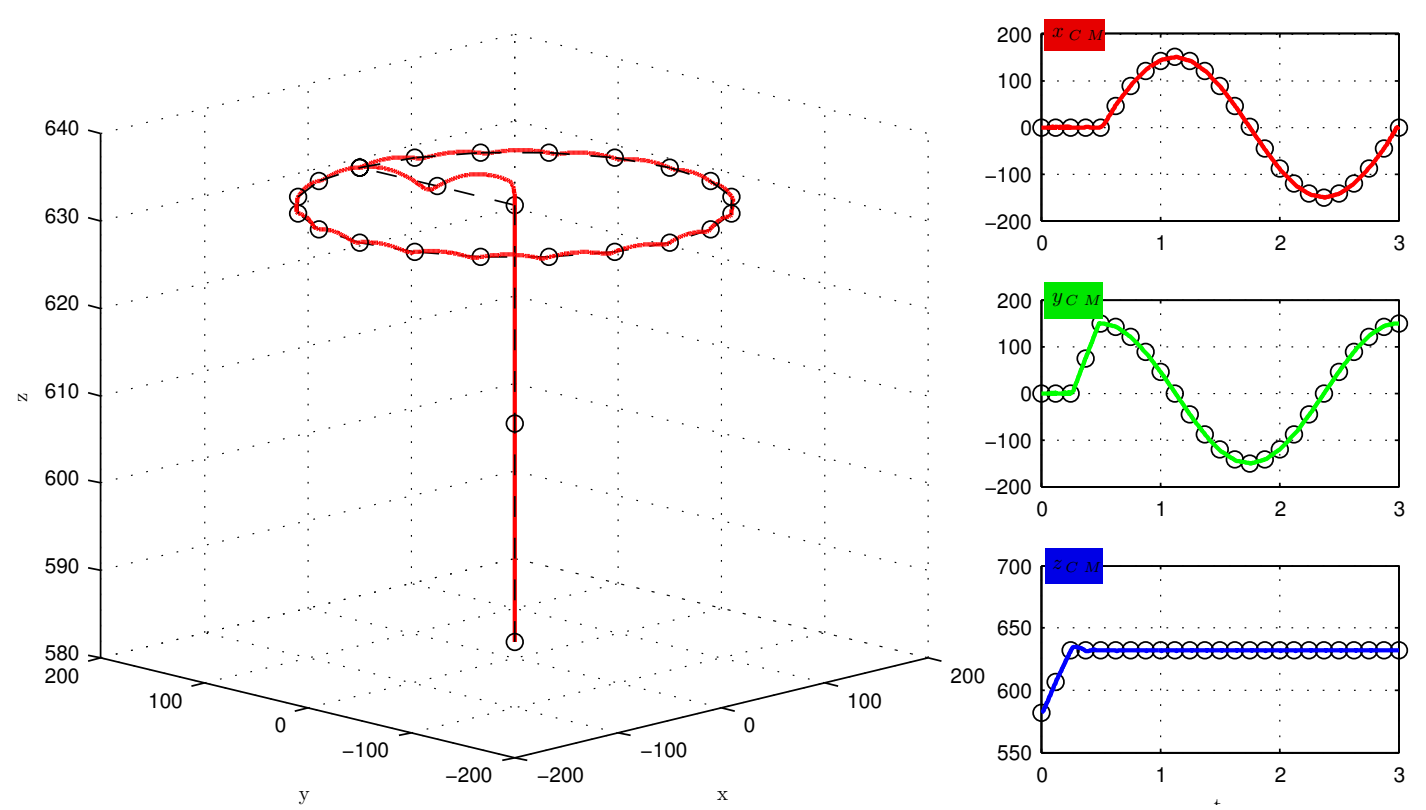

Figure 11.3: Movement II: Circular trajectory.

\subsection{Controllers}

In this section it is detailed all the approaches put into test in order to control the PM.

\subsubsection{Decoupled Controllers}

Naturally, the first approaches are based on the controllers presented in chapter 7 for the control of the SHA, and considering the challenge as a decoupled problem where the action of the actuators is tackled individually. This strategy also known as kinematic control is based on the fact that if the actuators follows the desired trajectory it is expected that the resulting movement of the moving platform is the desired.

The general architecture of this controller is presented in Fig.11.4. The first two blocks in the diagram corresponds to the trajectory generation described in the previous section.

Based on this architecture, it is implemented the $\mathrm{PI}+\mathrm{P}$ adaptive position-force controller, which was modified in order to receive the information from the force sensor integrated in the PM. 


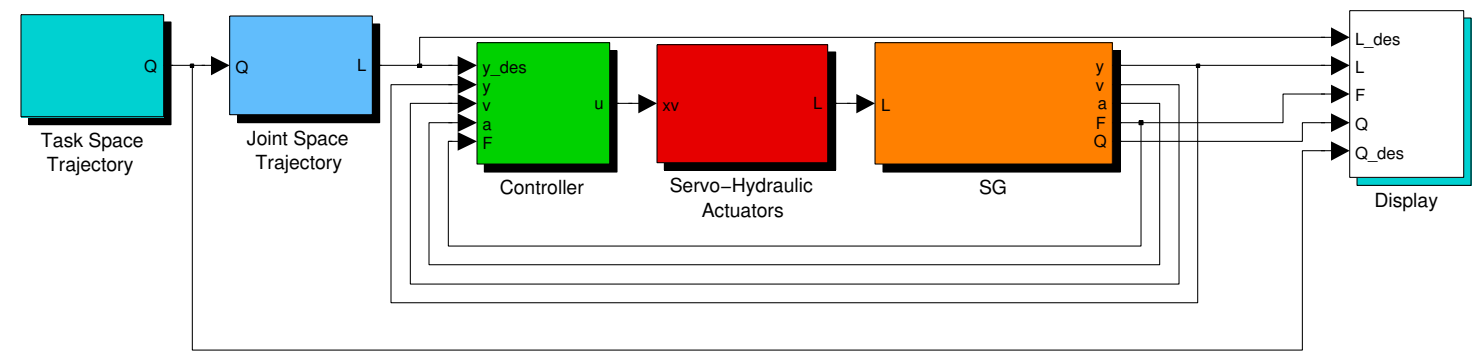

Figure 11.4: Schematic diagram for the SG joint space controller.

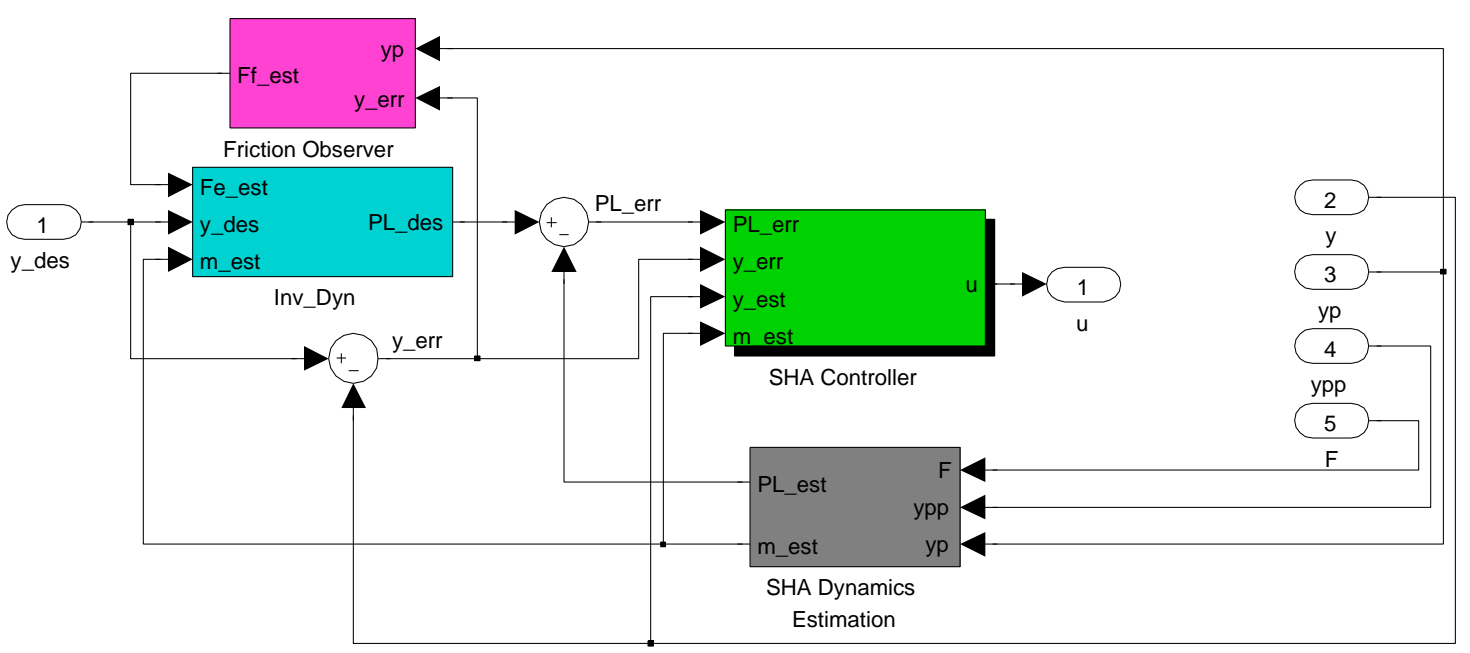

Figure 11.5: Implementation of the adaptive controller of the SHA in the SG.

\subsubsection{Dynamic Model Based Controller}

The $\mathrm{PI}+\mathrm{P}$ adaptive controller presented previously is modified in order to include a feedforward compensation as presented in Fig. 11.6. In this diagram it can be seen that modification affects the way that $P L_{\text {target }}$ is calculated, as a manner of fact it is given by:

$$
P_{L_{\text {target }}}=\frac{\tau_{i}+\hat{F}_{f}}{\Delta P}
$$

where $\tau_{i}$ is force obtained from the dynamic model (see Fig. 11.7.) 


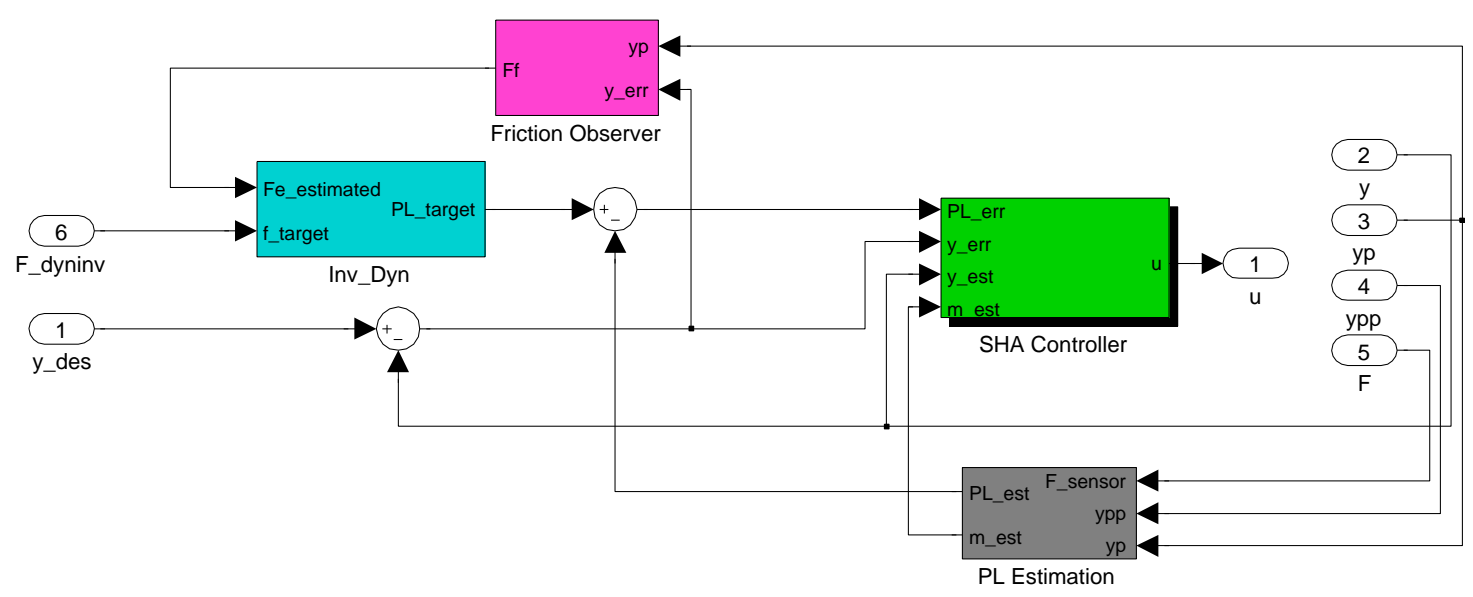

Figure 11.6: Joint space controller with feedforward compensation.

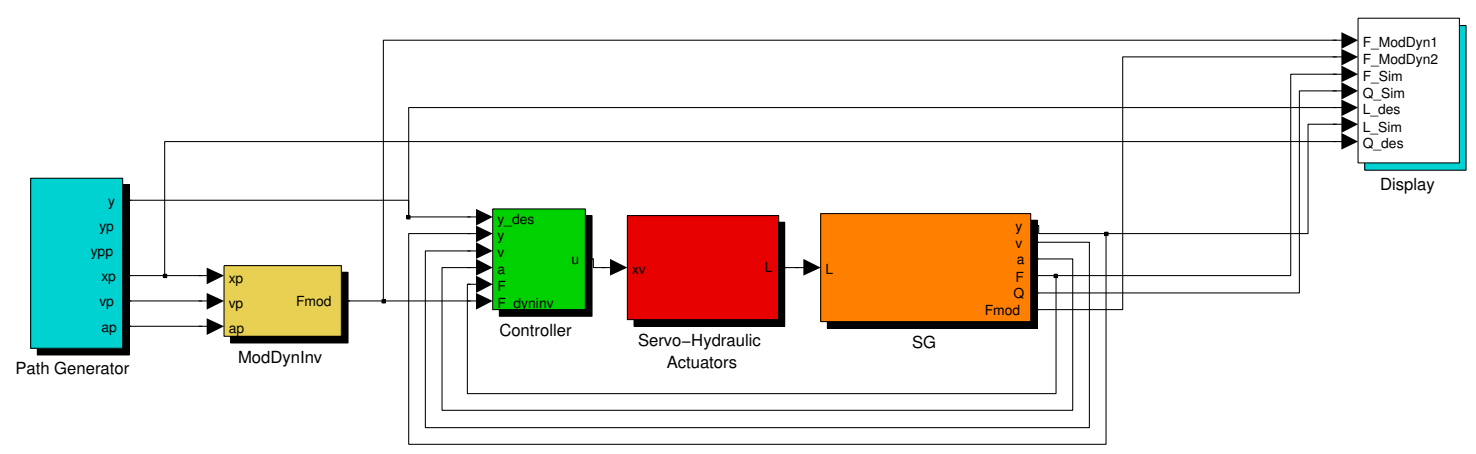

Figure 11.7: Joint space controller with feedforward compensation.

\subsection{Simulation and Results}

The controller under analysis were submitted to the two movements previously described.

For the first movement, i.e. point-to-point, it was defined an acceleration time of $t_{a}=50 \mathrm{~ms}$ and a transition from point to point of $t_{m}=400 \mathrm{~ms}$. The result for this movement is presented in Fig.11.8 (x, y and $\mathrm{z}$ coordinates of the end effector in the top, middle and bottom position respectively). The red curves shows the corresponding coordinate of the end effector using the model-based controller. The blue curves corresponds with the results implementing the adaptive PI+P controller. The black-dashed curve is the target position. In order to objectively quantify the performance of the 
controllers, it is also shown in the red and blue colored boxed the integral absolute error of the curves (IAE).

As it can be observed in these charts, both controllers follow the target position. Nevertheless, it can be appreciated that the model-based controller follows the target slightly better as it is also indicated in the IAE index. Additionally, the model-based controller presents a better response in the peak and valleys of the target position.
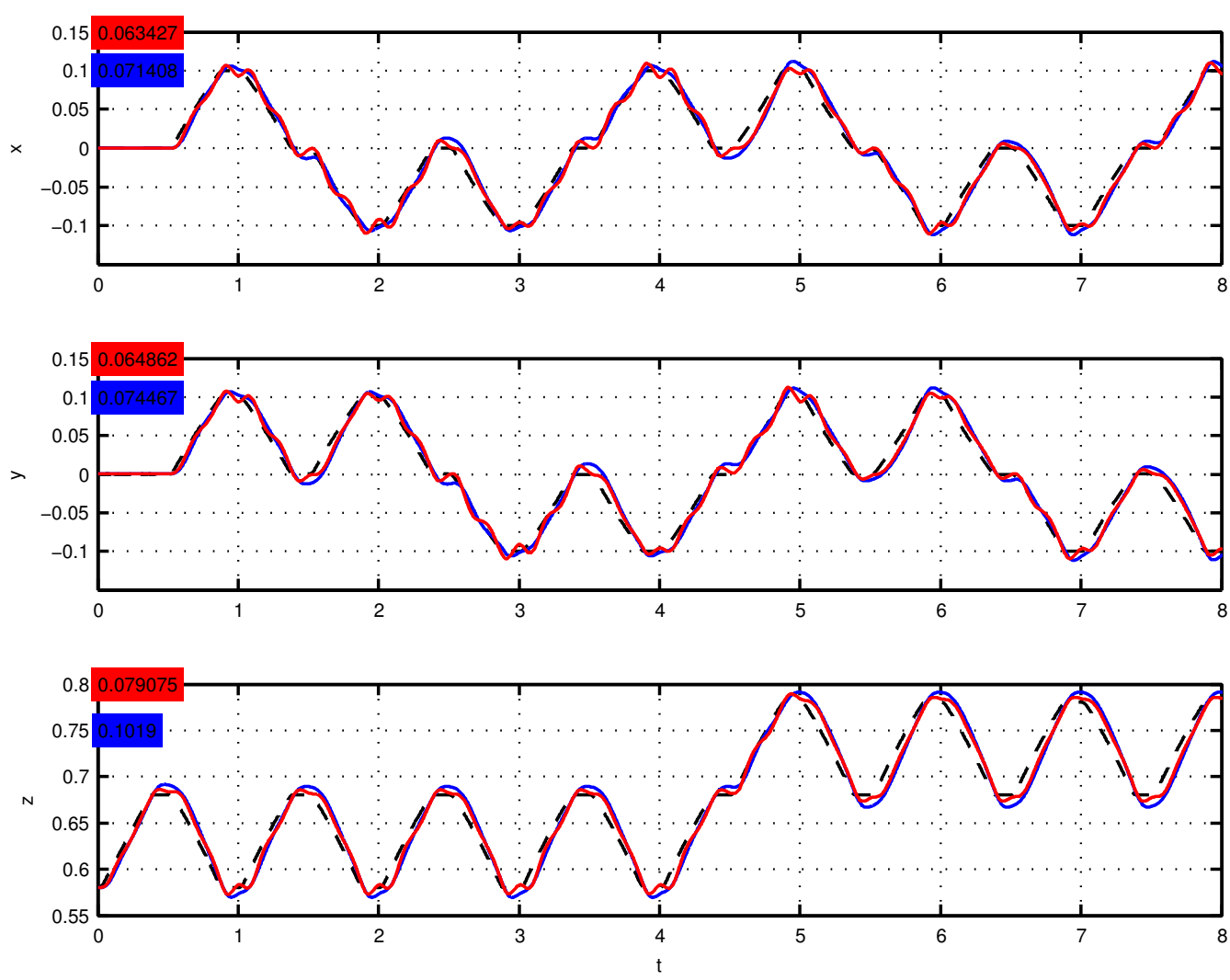

Figure 11.8: End effector position during the diagonal movement.

For the circular trajectory, it was also defined an acceleration time of $t_{a}=50 \mathrm{~ms}$ but the transition time was settled to $t_{m}=250 \mathrm{~ms}$. The result for this movement is presented in Fig. 11.9 following the same guidelines that the previous one, i.e.: red curves is the result from the implementation of the model-based controller and the blue curves correspond to the result with the adaptive $\mathrm{PI}+\mathrm{P}$ controller. The first portion of the circular trajectory corresponds to the movement of the end effector from its 
home position to the position from where the circular movement begins. As it can be observed in the charts, is in this transition were the major difference between the results from both controller occurs, then the difference is not appreciated. Considering the IAE index, once again the model based controller provides better performance.
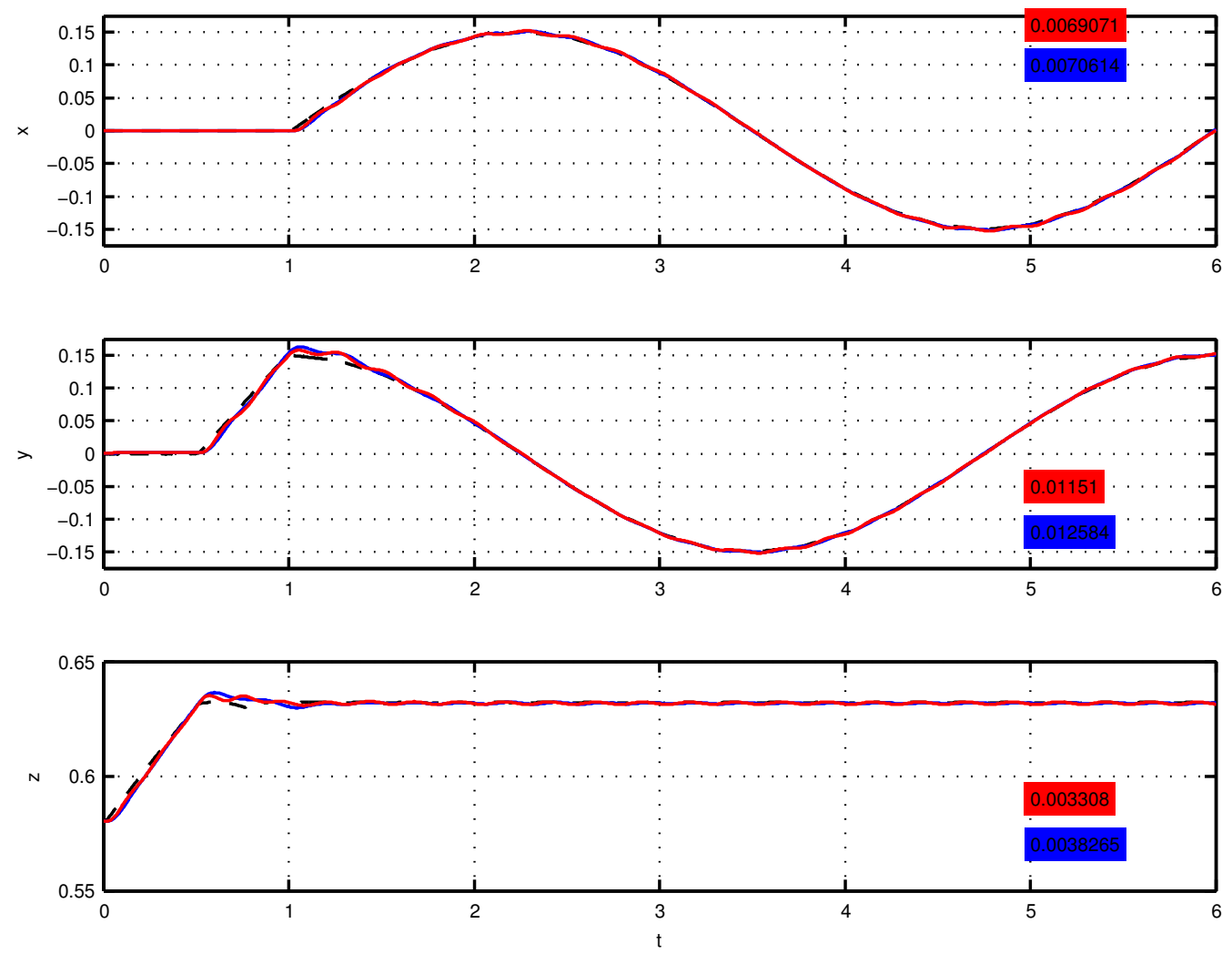

Figure 11.9: End effector position during circular movement.

\subsection{Conclusions}

The controller designed for the hydraulic actuator and presented during the second part of this book, can be extended to the rest of the prismatic actuator of the PM providing a simple monoarticular controller. As it was shows its performance, Even though it is not the best and there still room for improvements. The feedforward compensation, presents some enhancements regarding the overall path tracking. 


\section{Conclusions}

“... time traveling is just too dangerous. Better I devote myself to study the other great mystery of the universe: women ..."

Doc Emmet Brown.

In this thesis is been addressed all the aspects of the elements involved in control: kinematic and dynamic modeling, model identification, estate estimation, trajectory planing and control per se.

The system targeted for control is the classical hexapod parallel mechanism (normally refereed as the Stewart-Gough mechanism) powered by hydraulic actuator. In general parallel mechanisms represents a real challenge from the point of view of control given their intrinsic nonlinear and highly coupled dynamic characteristic. Furthermore, the overall nonlinear behavior of the system is worsened by the fact that the manipulator is powered with an hydraulic actuation system. Therefore in order to overcome with this first challenge the actuation system must be analyzed in depth.

Based on the governing dynamics of the hydraulic actuator a linearized expression was found, revealing two distinctive parts. The first one is a direct relation between the input and output variable, the other one shows a relation between external perturbation and the output variable. In general, this last one is left apart and it is assumed that the control algorithm will neutralize its effect. This assumption may lead to low performance controller. Additionally the linearized model is a Type-I system, i.e. the 
transfer function that relates the command input and the output position of the cylinder has a pole in the origin of the complex plane, which makes the implementation of PI position controller controversial. Nevertheless, as it was demonstrated in this work by means of the small gain theorem, PI control can provide robustness to the system.

The linearized model of the hydraulic system represent the governing dynamic the actuator, which must be customized by finding the values of the parameters involved in the equations. Even though some of this parameters may be found in catalogs and given their highly dependency to the environmental conditions, they must be identified on the system. The experimental identification process is complex and time consuming. It is not straight forward and it requires a certain level of expertise to adopt the appropriate criteria.

On the other hand, linearized models are a simplified set of equation that represents the dynamic behavior of a system for a particular working condition. In order to span the overall dynamic of the system, the linearization must be performed for several working point, resulting in a system with parameter that varies with the working condition. The variation of the parameters for each working point, gives an idea of the parametric uncertainty of the model.

Considering the hydraulic actuator as a system composed by several elements that are in relative movement between them, there is one physical effects that compromise its performance and that is the friction forces. This effect, that it is also nonlinear, might be represented by several models and approaches. From all these approaches, the LuGre model is the one that is capable of reproducing more behaviors (static friction, stick-slip, Coulomb friction, viscous friction, time-lag, oscillation, etc). The experimental identification of the parameters that characterize the force friction must be performed specifically for each parameter, i.e. some parameters are obtained under constant velocity movement and some of them are obtained under force controlled movement.

From the control point of view it was demonstrated theoretically and experimentally that PD and PID controllers may lead to instability of the system and that the performance the controller is enhanced by considering a gain-scheduling strategy, where the parameters of the controller are tuned specifically for each working point. Furthermore, the inclusion of a Force loop that compensate the friction forces provides better results.

Putting back the hydraulic actuation system in the parallel manipulator, and considering the whole system to be controlled, it must be highlighted two important factor of the mechanism: its kinematics and dynamics. The first one, is mostly related with 
the constraints of the mechanism such as workspace, singularity conditions, etc. That are necessary to keep in mind while planing the trajectories to follow. Second, the dynamics of the system that help to improve the performance of the controller.

The basic kinematic analysis of the mechanism, comprises the analysis of its workspace and presence of singular configuration. The latter one, can be obtained from the traditional approach of differentiate de kinematic model, or it can be obtained using a more sophisticate tool as the Screw theory" method, which provides a better approach for analysis.

The dynamic model of a parallel manipulator is highly needed to be part of the control loop in application that require high precision and performance. In this the virtual work is implemented.

Considering the whole system, the controllers implemented for the hydraulic actuator as individual element are scaled-up for the entire mechanism. This approach is based on the fact that if the corresponding trajectory of the actuator are accurately followed, the moving platform should also follow the desired trajectory in the state space.

The study of control algorithm is a non ending topic that provides many alternatives. As a matter of fact, as a future work it is proposed to develop and $H_{\text {infty }}$ controller for the hydraulic actuator based on the variability of the parameters extending the results presented with the PI controller. It can also be study the robustness of the $\mathrm{PI}+\mathrm{P}$ adaptive controller. The most challenging aspect, will be to develop a task space controller, and test all the strategies in the real manipulator. 
12. CONCLUSIONS 


\section{Appendix A}

\section{Parameters of the Identification of the HSA}

Table A.1: Parameters of motion

\begin{tabular}{r|ccc|ccc|ccc|}
$P_{o}$ & $m_{0 k}$ & $\sigma_{0 k}$ & $F_{e 0 k}$ & $m_{3 k}$ & $\sigma_{3 k}$ & $F_{e 3 k}$ & $m_{6 k}$ & $\sigma_{6 k}$ & $F_{e 6 k}$ \\
\hline \hline 10 & 2.57 & 27.69 & 28.8 & 4.87 & 29.97 & 46.56 & 7.28 & 37.66 & 68.67 \\
30 & 2.41 & 24.31 & 26.42 & 4.87 & 26.97 & 45.9 & 7.27 & 33.93 & 68.57 \\
60 & 2.22 & 20.12 & 23.44 & 4.87 & 23.38 & 45.1 & 7.26 & 29.38 & 68.49 \\
90 & 2.08 & 17 & 21.19 & 4.89 & 20.89 & 44.55 & 7.27 & 26.11 & 68.49 \\
120 & 1.98 & 14.96 & 19.66 & 4.92 & 19.49 & 44.24 & 7.29 & 24.12 & 68.58 \\
150 & 1.93 & 13.99 & 18.85 & 4.95 & 19.2 & 44.17 & 7.33 & 23.4 & 68.76 \\
180 & 1.93 & 14.09 & 18.77 & 5 & 20.01 & 44.34 & 7.38 & 23.96 & 69.03 \\
210 & 1.98 & 15.26 & 19.41 & 5.05 & 21.91 & 44.76 & 7.46 & 25.8 & 69.38 \\
240 & 2.08 & 17.5 & 20.77 & 5.12 & 24.91 & 45.41 & 7.54 & 28.91 & 69.81 \\
270 & 2.23 & 20.82 & 22.86 & 5.19 & 29.02 & 46.31 & 7.65 & 33.29 & 70.34 \\
290 & 2.35 & 23.62 & 24.66 & 5.24 & 32.36 & 47.04 & 7.72 & 36.93 & 70.74
\end{tabular}


Table A.2: Sensibility Constants

\begin{tabular}{r|ccc|ccc|ccc|}
$P_{o}$ & $K_{x 0 k}$ & $K_{d 0 k}$ & $K_{p_{0 k}}$ & $K_{x 3 k}$ & $K_{d 3 k}$ & $K_{p_{0 k}}$ & $K_{x 6 k}$ & $K_{d 6 k}$ & $K_{p_{6 k}}$ \\
\hline \hline 10 & 71690 & 244 & -117 & 115886 & 399 & -104 & 145261 & 536 & -96 \\
30 & 62876 & 221 & -112 & 105248 & 382 & -101 & 130609 & 502 & -93 \\
60 & 52427 & 193 & -107 & 93220 & 363 & -98 & 113806 & 462 & -90 \\
90 & 45303 & 175 & -103 & 85909 & 353 & -95 & 103211 & 437 & -88 \\
120 & 41503 & 164 & -100 & 83314 & 352 & -94 & 98826 & 425 & -87 \\
150 & 41029 & 163 & -99 & 85436 & 360 & -94 & 100650 & 428 & -87 \\
180 & 43880 & 170 & -99 & 92273 & 376 & -94 & 108683 & 445 & -88 \\
210 & 50055 & 186 & -101 & 103827 & 401 & -96 & 122925 & 475 & -89 \\
240 & 59556 & 211 & -104 & 120097 & 435 & -99 & 143376 & 520 & -92 \\
270 & 72382 & 245 & -108 & 141084 & 477 & -103 & 170037 & 579 & -95 \\
290 & 82780 & 272 & -111 & 157695 & 510 & -106 & 191260 & 625 & -98
\end{tabular}

Table A.3: Parameter of the Transfer Functions

\begin{tabular}{r|ccc|ccc|ccc|}
$P_{o}$ & $b_{00 k}$ & $b_{10 k}$ & $k_{010 k}$ & $b_{03 k}$ & $b_{13 k}$ & $k_{013 k}$ & $b_{06 k}$ & $b_{16 k}$ & $k_{016 k}$ \\
\hline \hline 10 & 13345 & 128 & 266 & 11040 & 110 & 274 & 9837 & 101 & 258 \\
30 & 12771 & 122 & 259 & 10515 & 106 & 261 & 9201 & 98 & 248 \\
60 & 12036 & 116 & 249 & 9929 & 102 & 245 & 8453 & 94 & 236 \\
90 & 11522 & 111 & 241 & 9583 & 99 & 233 & 7952 & 92 & 227 \\
120 & 11302 & 108 & 236 & 9471 & 98 & 227 & 7701 & 90 & 224 \\
150 & 11425 & 106 & 236 & 9591 & 97 & 229 & 7696 & 90 & 227 \\
180 & 11905 & 107 & 242 & 9936 & 98 & 236 & 7933 & 91 & 236 \\
210 & 12706 & 108 & 252 & 10498 & 100 & 249 & 8405 & 93 & 249 \\
240 & 13754 & 112 & 264 & 11270 & 104 & 265 & 9106 & 96 & 265 \\
270 & 14956 & 117 & 276 & 12243 & 108 & 282 & 10024 & 100 & 282 \\
290 & 15799 & 121 & 283 & 13000 & 112 & 294 & 10751 & 103 & 292
\end{tabular}


Appendix B

Well-posedness of the feedback System 


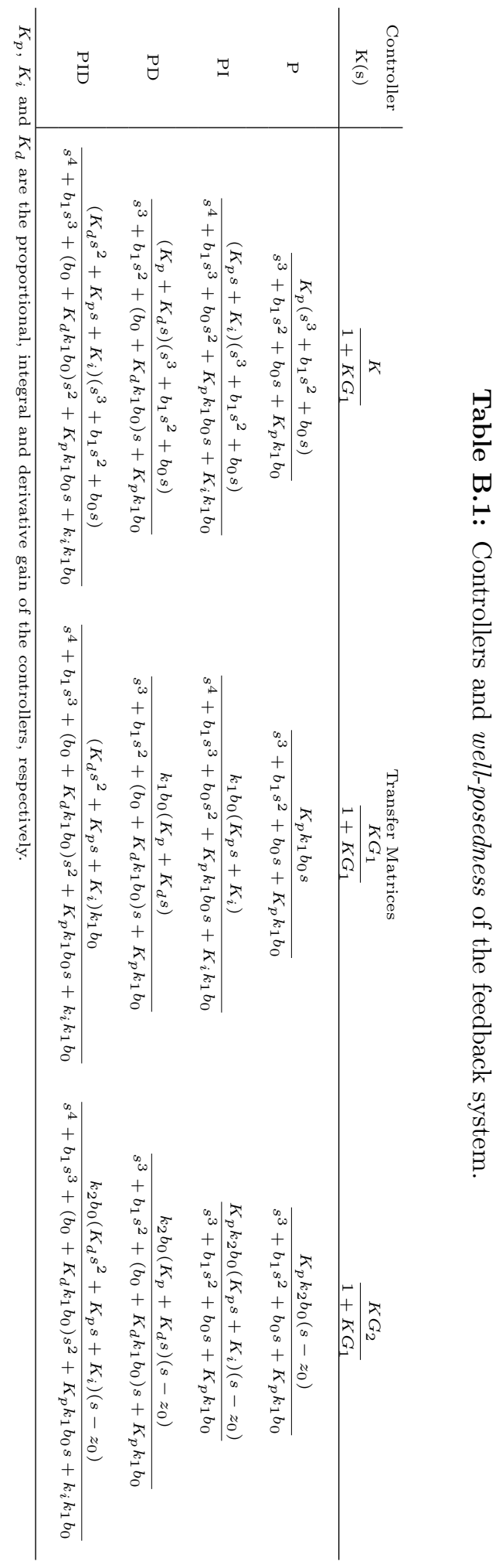




\section{Appendix C}

\section{Virtual rotations and Euler parameters}

Let us suppose a point $\mathrm{S}$ attached to a moving frame $O_{x^{\prime} y^{\prime} z^{\prime}}^{\prime}$ whose origin is coincident with the origin of a fixed frame $O_{x y z}$. The orientation of the moving frame expressed in the fixed frame is given by the rotation matrix $\boldsymbol{R}$. The location of point $\mathrm{S}$ expressed in the moving frame is given by the vector $s^{\prime}$, and it can be expressed on the fixed frame as follows:

$$
s=R s^{\prime}
$$

Let us now consider that $O_{x^{\prime} y^{\prime} z^{\prime}}^{\prime}$ is slightly perturbed, which traduces into a change in the location of point $\mathrm{S}$ as follows:

$$
\delta s=\delta \boldsymbol{R} \boldsymbol{s}^{\prime}
$$

the operator $\delta$, called infinitesimals, may be interpreted as a partial differentials operator, which allows to define the following identities (67).

From the orthogonal properties of a rotation matrix $\boldsymbol{R} \boldsymbol{R}^{T}=\boldsymbol{I}$, it is derived the definition of the virtual rotation of the $O_{x^{\prime} y^{\prime} z^{\prime}}^{\prime}$ frame relative to the $O_{x y z}$ with component in the latter frame as follows:

$$
\widetilde{\delta \boldsymbol{\pi}}=\delta \boldsymbol{R} \boldsymbol{R}^{T}
$$

After some algebraic manipulation, it can be shown that:

$$
\delta \boldsymbol{R}=\boldsymbol{R} \widetilde{\delta \boldsymbol{\pi}^{\prime}}
$$

where $\delta \boldsymbol{\pi}^{\prime}$ is the virtual rotation expressed in $O_{x^{\prime} y^{\prime} z^{\prime}}^{\prime}$ 
Therefore the changes on the generalized coordinates (i.e. $\delta \boldsymbol{s}$ ) can be expressed in terms of the virtual displacements as follows:

$$
\delta s=R \widetilde{\delta^{\prime}} s^{\prime}
$$

by properties of skew matrices:

$$
\delta s=-\boldsymbol{R} \widetilde{s^{\prime}} \delta \pi^{\prime}
$$

On the other hand, it can be proved that $\boldsymbol{R}=\boldsymbol{E} \boldsymbol{G}^{T}$ and $\delta \boldsymbol{R}=2 \boldsymbol{E} \delta \boldsymbol{G}^{T}$, where $\boldsymbol{E}=\left[\boldsymbol{e}, \tilde{\boldsymbol{e}}+e_{0} \boldsymbol{I}\right]$ and $\boldsymbol{G}=\left[\boldsymbol{e},-\tilde{\boldsymbol{e}}+e_{0} \boldsymbol{I}\right]$, are identities matrices, where $\boldsymbol{e}=\left[e_{1}, e_{2}, e_{3}\right]$.

Therefore,

$$
\begin{aligned}
\widetilde{\delta \boldsymbol{\pi}^{\prime}} & =\boldsymbol{R}^{T} \delta \boldsymbol{R}, \\
& =\boldsymbol{E} \boldsymbol{G}^{T} 2 \boldsymbol{E} \delta \boldsymbol{G}^{T}, \\
& =2 \boldsymbol{E} \boldsymbol{E}^{T} \boldsymbol{G} \delta \boldsymbol{G}^{T}, \\
& =2 \boldsymbol{G} \delta \boldsymbol{G}^{T} .
\end{aligned}
$$

Considering the definition of $\boldsymbol{G}$ and $\boldsymbol{p}$ it can also be demonstrated that $\boldsymbol{G} \delta \boldsymbol{G}^{T}=$ $\widetilde{\boldsymbol{G} \delta \boldsymbol{p}}$, and thus $\delta \boldsymbol{\pi}^{\prime}=2 \boldsymbol{G} \delta \boldsymbol{p}$.

Taking this last result into (C.6), the relation between the changes of the generalize coordinate of vector $s$ due to small perturbations of the euler parameters can be stated as follows:

$$
\delta s=-2 \boldsymbol{R} \widetilde{s^{\prime}} \boldsymbol{G} \delta p
$$




\section{Appendix D}

\section{Estimation of the Dynamics of theTestbed}

The linear guide integrated in the Testbed posses its own dynamic, that has to be considered and removed during the identification procedure of the SHA. Therefore, the dynamics of the linear guide, which is mainly governed by the friction between its elements, has to be determined first.

Thus, given the characteristics and disposition of the elements of the linear guide (see Fig. ??), its dynamics can be estimated from experimentation by performing free-fall of the sliding element. Based on the consideration that the sliding element is only affected by its weight $\left(g m_{T}\right)$ and the friction force $\left(F_{f}\right)$ of the linear guide, the dynamics of the moving element can be stated as,

$$
g m_{T}-F_{f}(t)=\ddot{y}(t) m_{T}
$$

and equating (D.1), the friction force of the linear guide is given by,

$$
F_{f}(t)=(g-\ddot{y}(t)) m_{T},
$$

where $g=-9.81\left[\mathrm{~m} / \mathrm{s}^{2}\right]$ is the gravity acceleration constant, $m_{T}=m_{0}+m_{\text {ext }}$, is the total mass in $[K g]$, given by the sliding element $\left(m_{0}\right)$ and the added load $\left(m_{e x t}\right) ; F_{f}$ is the friction force, and $\ddot{y}(t)$ is the acceleration of the sliding element.

Therefore, ten free-fall experiments for different external loads where performed. During this experiments, the position of the sliding element was registered. The velocity and acceleration are obtained from the position of the sliding element implementing a finite differentiation and a 4th order lowpass Butterworth filter with a cut-off frequency: $w_{c}=100[H z]$, and the friction force is calculated using (D.2). The force-velocity characteristic of the system is presented in Fig. D.1. 


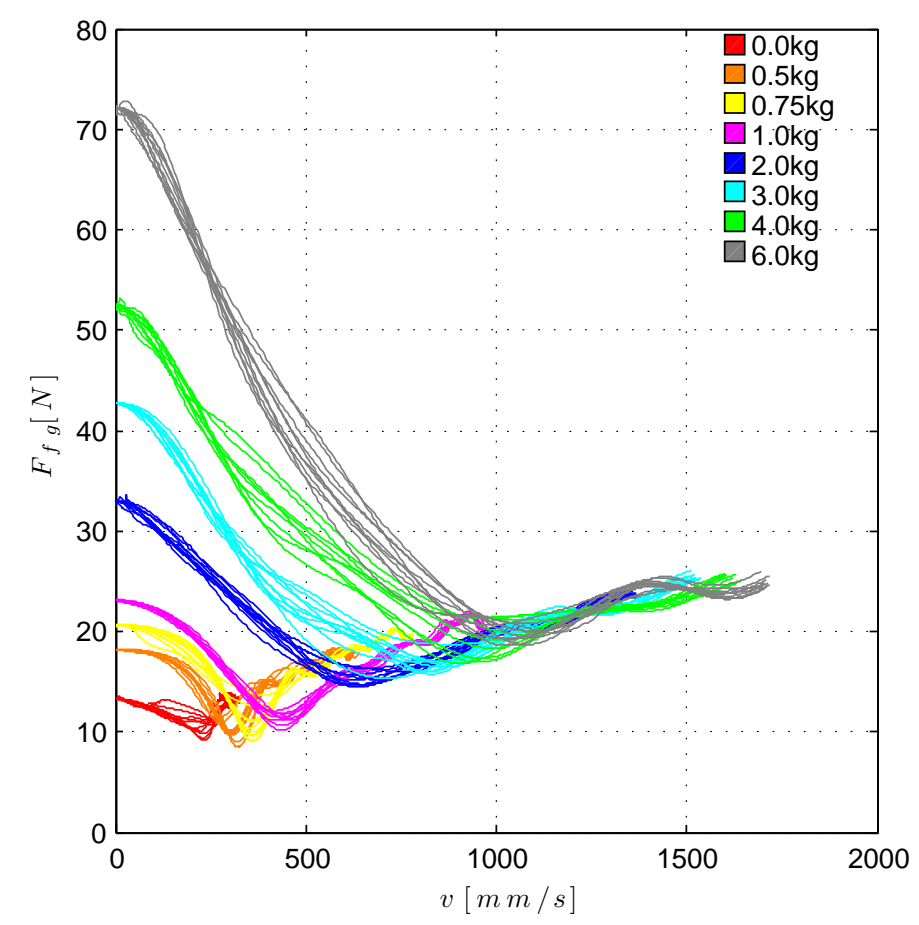

Figure D.1: Force-velocity characteristic of the linear guide from free-fall experimentation.

As it can be observed, the force-velocity characteristic of the linear guide shows the well known behavior of systems with friction. Thus, a conventional friction model is chosen in order to estimate the dynamics of the linear guide. The Lu-Gre model could be adequate for this purpose. However, given the characteristics of the free-fall experiments (i.e. no controlled velocity nor constant velocity), a simpler static friction model given by (D.3), is considered instead.

$$
F(v)=F_{c}+\left(F_{s}-F_{c}\right) e^{-\left|v / v_{s}\right|^{\delta_{s}}}+\sigma_{v} v,
$$

where $F_{c}$ is the coulomb force, $F_{s}$ is the static force, $v_{s}$ is the Stribeck velocity, and $\sigma_{v} v$ defines the viscous force. It must be noticed that $F_{c}, v_{S}, F_{s}$ depends on the external load.

In friction-steady state behavior, i.e. when the effect of the exponential term of (D.3) dissipates, the dynamics will be governed by

$$
F_{s s}=F_{c}+\sigma_{v} v,
$$

which defines the viscous friction characteristic. This behavior can be estimated graph- 
ically from the results by eliminating the stribeck effect region, and implementing a first order curve fitting, as shown in Fig. D.2(a). The Stribeck velocity $\left(v_{s}\right)$, is defined as the intersection of the mean curve for each external load and the linear fitting of the viscous friction region (see Fig. D.2(b). The parameter estimated are summed up in Table D.1.

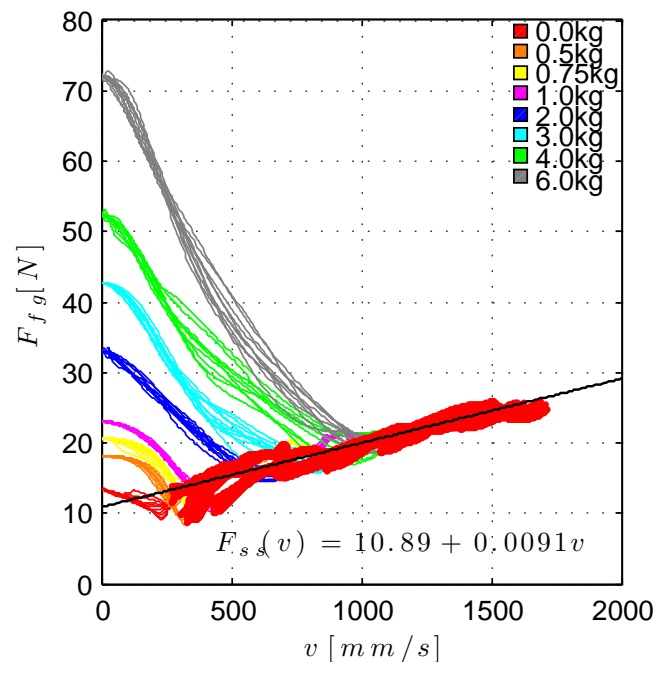

(a)

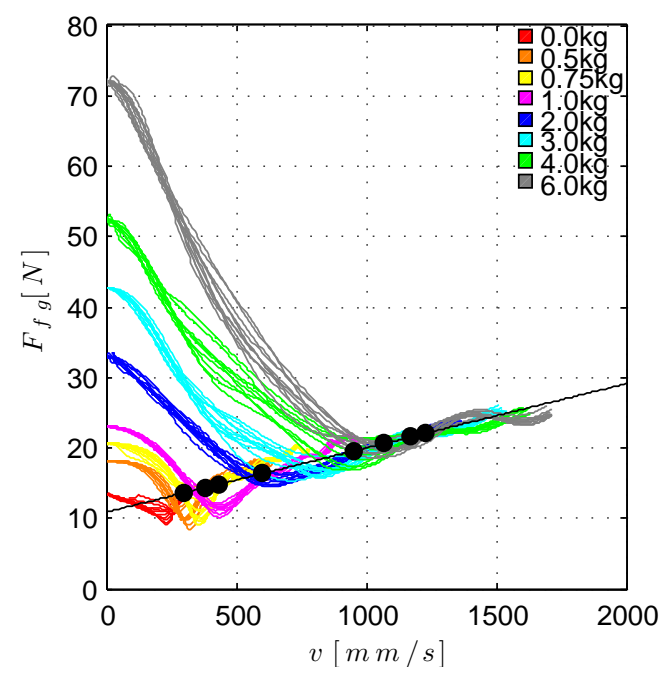

(b)

Figure D.2: Estimation of the parameter of the Force-velocity model of the linear guide. D.2(a): linear fitting of the viscous friction region. D.2(b): estimation of the Stribeck velocity.

Therefore, based on (D.3) with the parameters presented in Table D.1, the dynamic of the linear guide is estimated and presented in Fig. D.3.

It can be observed that the estimation based on the friction model presents inaccuracies in the Stribeck friction region, and the exponential characteristic of the model does not provide the complete information of the dynamics of the linear guide. Therefore, the Stribeck region is estimated implementing an unbounded spline with the data obtained experimentally. These splines are concatenated with the viscous friction model (D.4), at the velocity given by $v_{s}$ from Table D.1. This new estimation, which is shown in Fig.D.4, presents a better approach to the dynamics of the linear guide.

Implementing unbounded splines with the data estimated and the data obtained experimentally, the dynamics of the linear guide for external load of $[0,10] \mathrm{Kg}$ is estimated and presented as a surface in Fig. D.5. 
Table D.1: Parameters estimated for the force-velocity model of the linear guide

\begin{tabular}{cccccc}
${ }^{*} M_{e x t}[K g]$ & $v_{s}[\mathrm{~mm} / \mathrm{s}]$ & $F_{s}[N]$ & $F_{c}[N]$ & $\sigma_{v}[N s / m m]$ & $\delta_{s}$ \\
\hline \hline 0 & 300 & 13.3 & 10.9 & 0.0091 & 3 \\
0.5 & 380 & 18.2 & 10.9 & 0.0091 & 3 \\
0.75 & 430 & 20.6 & 10.9 & 0.0091 & 3 \\
1 & 600 & 23.1 & 10.9 & 0.0091 & 3 \\
2 & 950 & 32.9 & 10.9 & 0.0091 & 3 \\
3 & 1070 & 42.7 & 10.9 & 0.0091 & 3 \\
4 & 1170 & 52.5 & 10.9 & 0.0091 & 3 \\
6 & 1230 & 72.1 & 10.9 & 0.0091 & 3 \\
\hline
\end{tabular}

*The sliding element posses a weight of $1.35[\mathrm{Kg}]$.

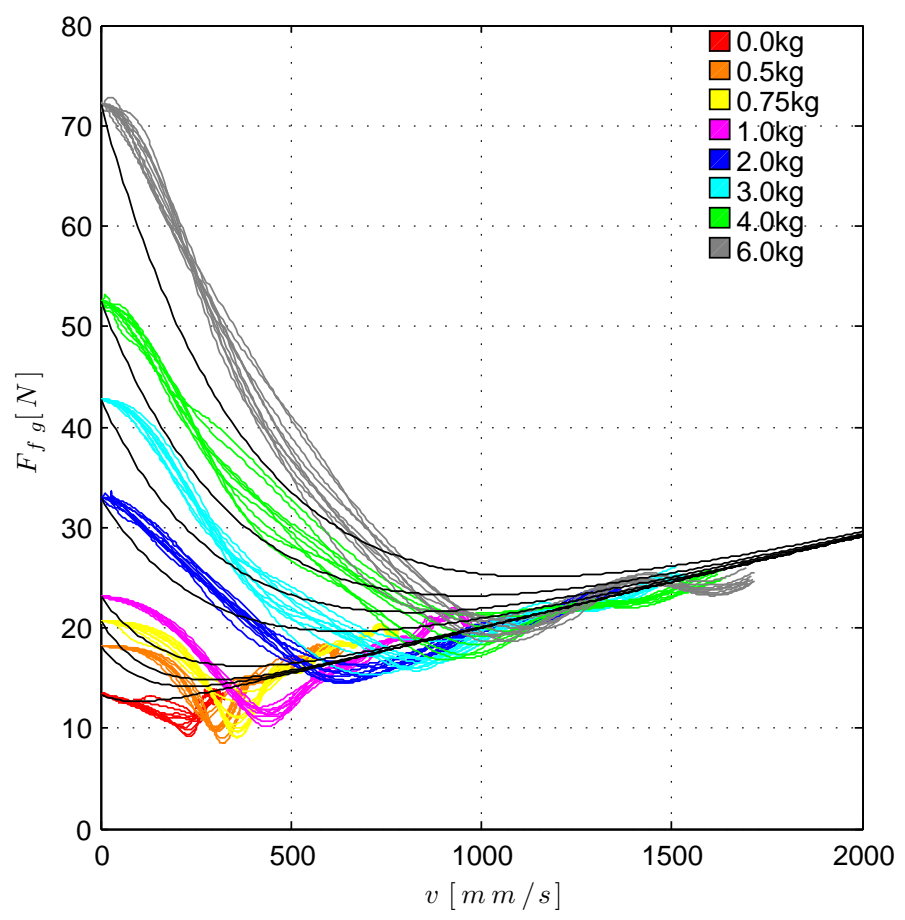

Figure D.3: Force-velocity estimation based on friction model. 


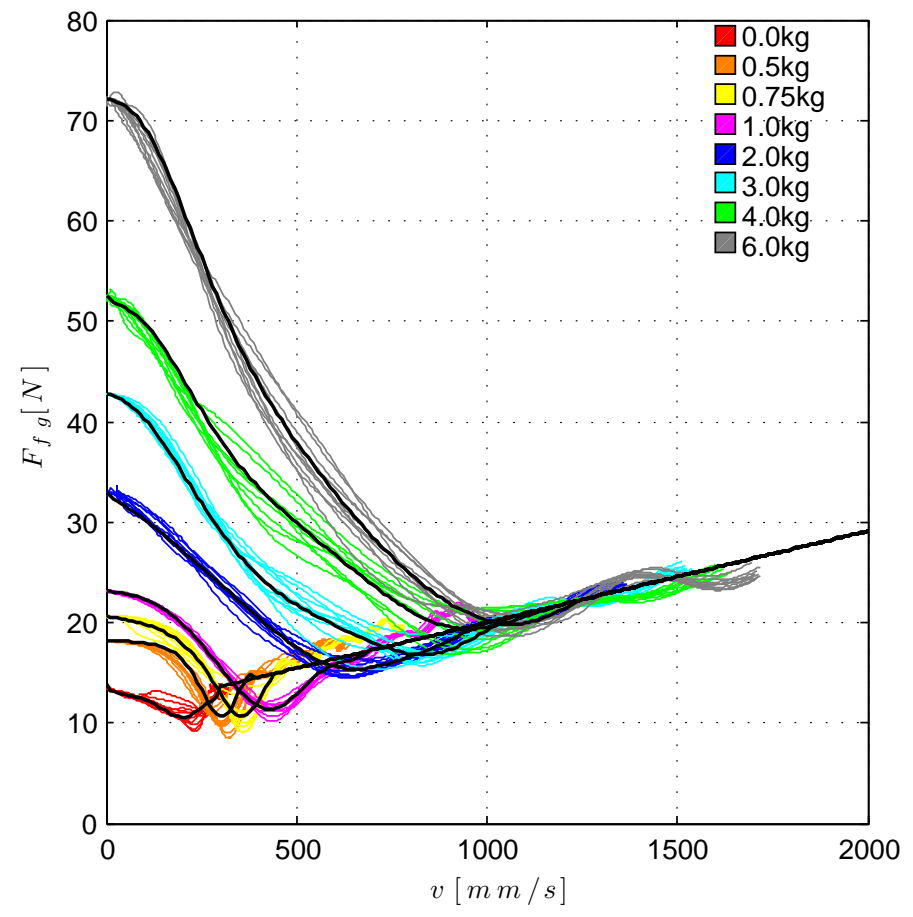

Figure D.4: Force-velocity estimation based on unbounded spline interpolation. 


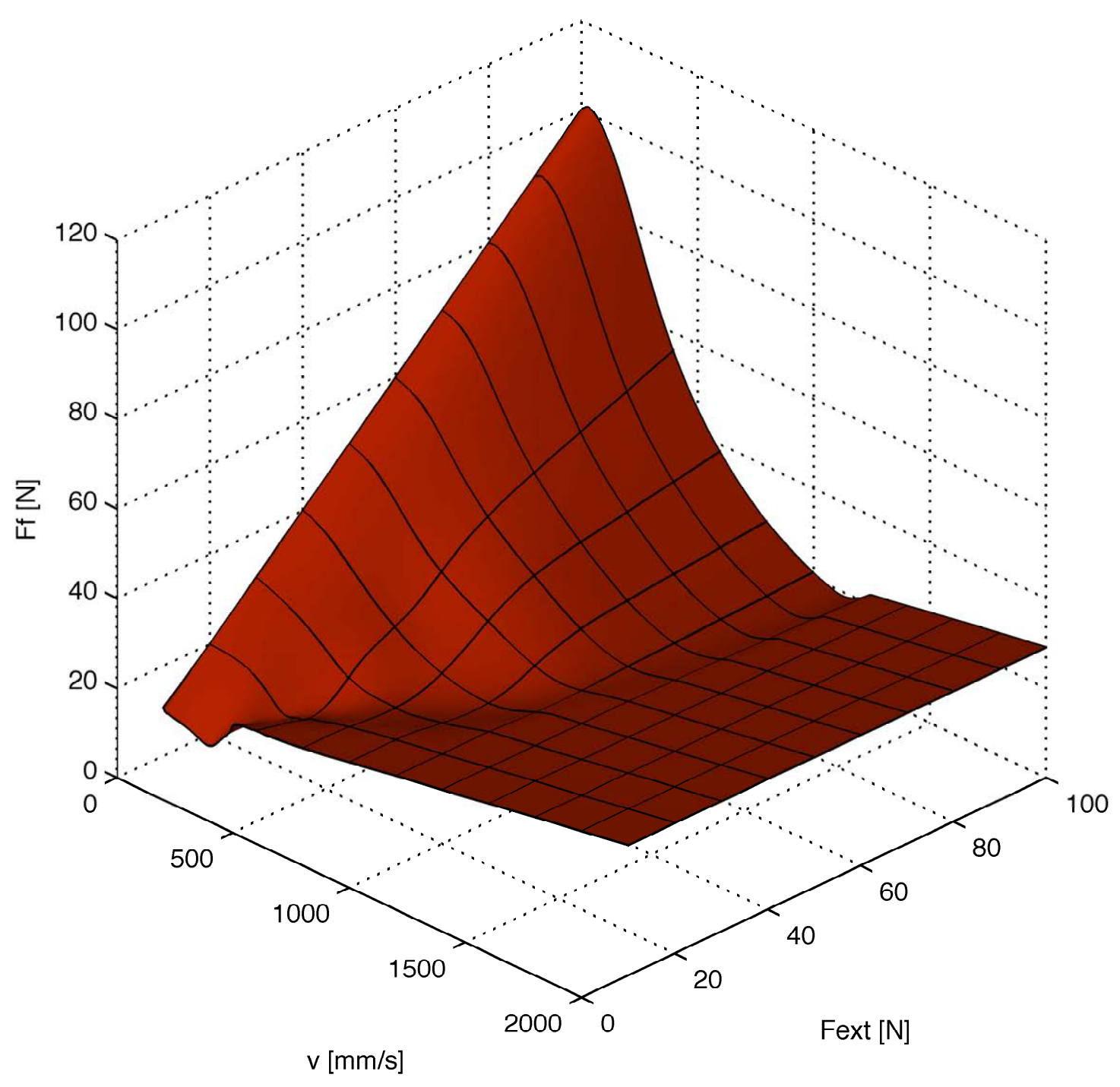

Figure D.5: Force-velocity characterization of the linear guide. 
Appendix E

Results from the validation of the dynamic model of the PM 

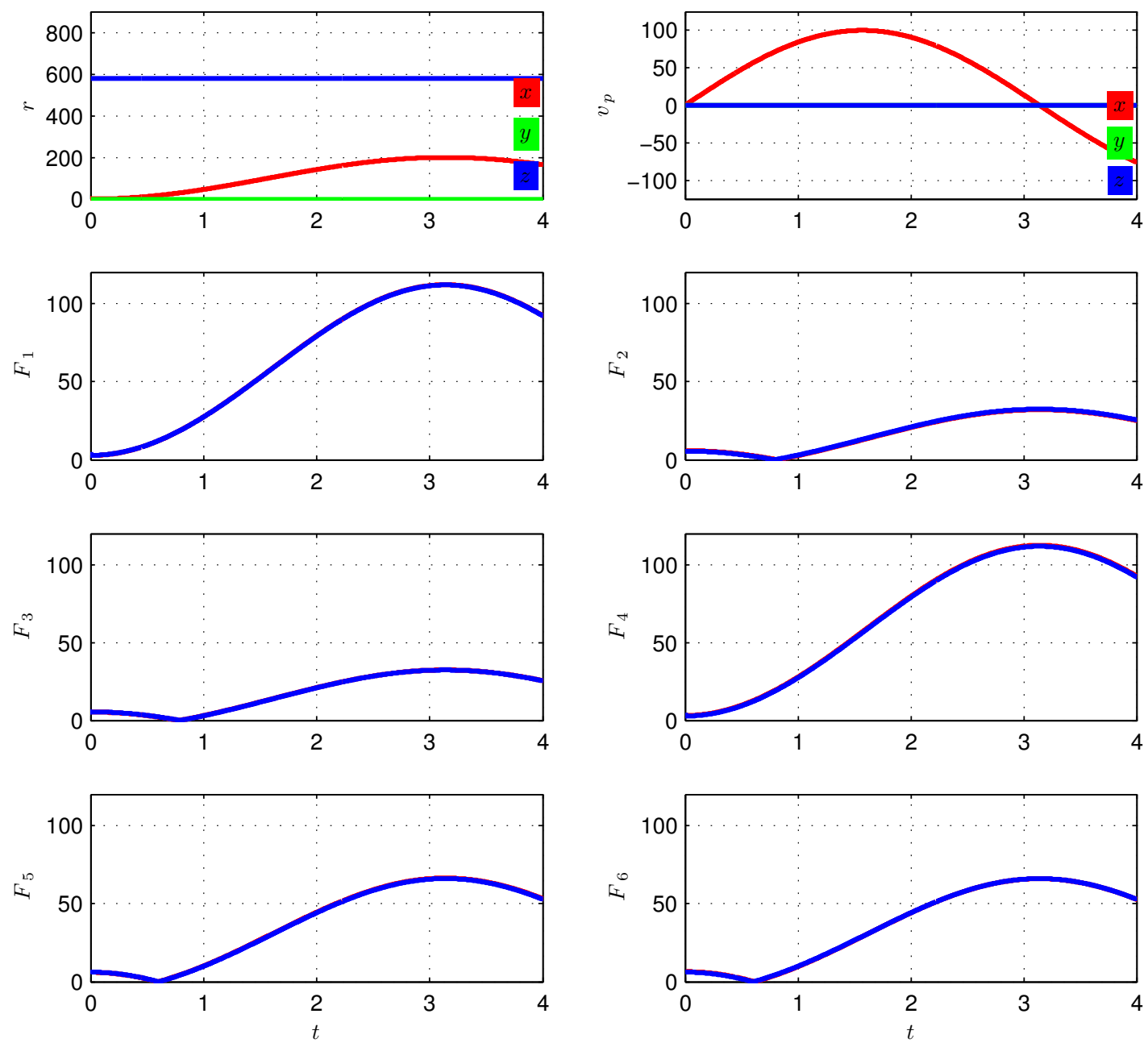

Figure E.1: Dynamic Model Validation, trajectory Tx. 

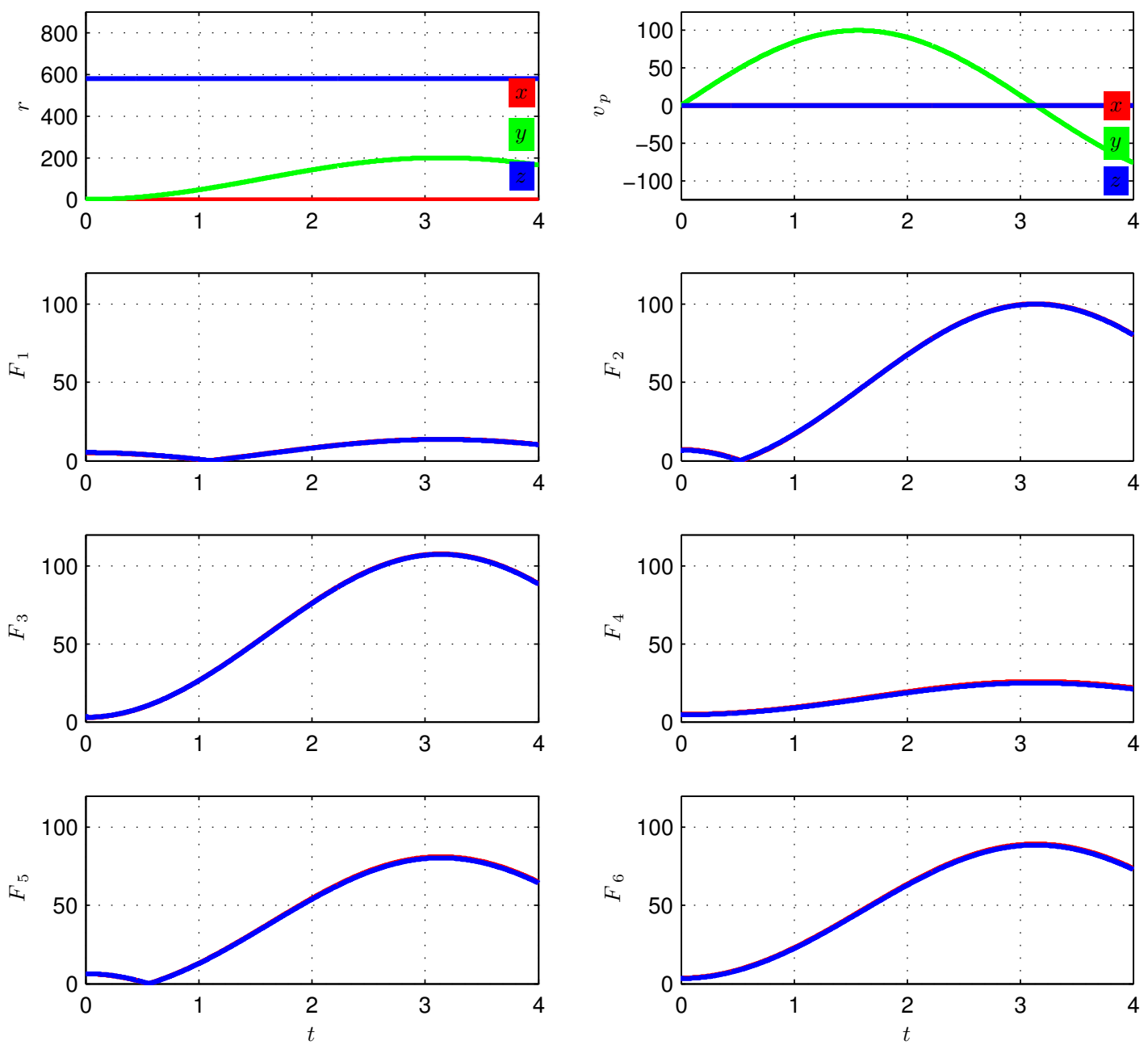

Figure E.2: Dynamic Model Validation, trajectory Ty. 

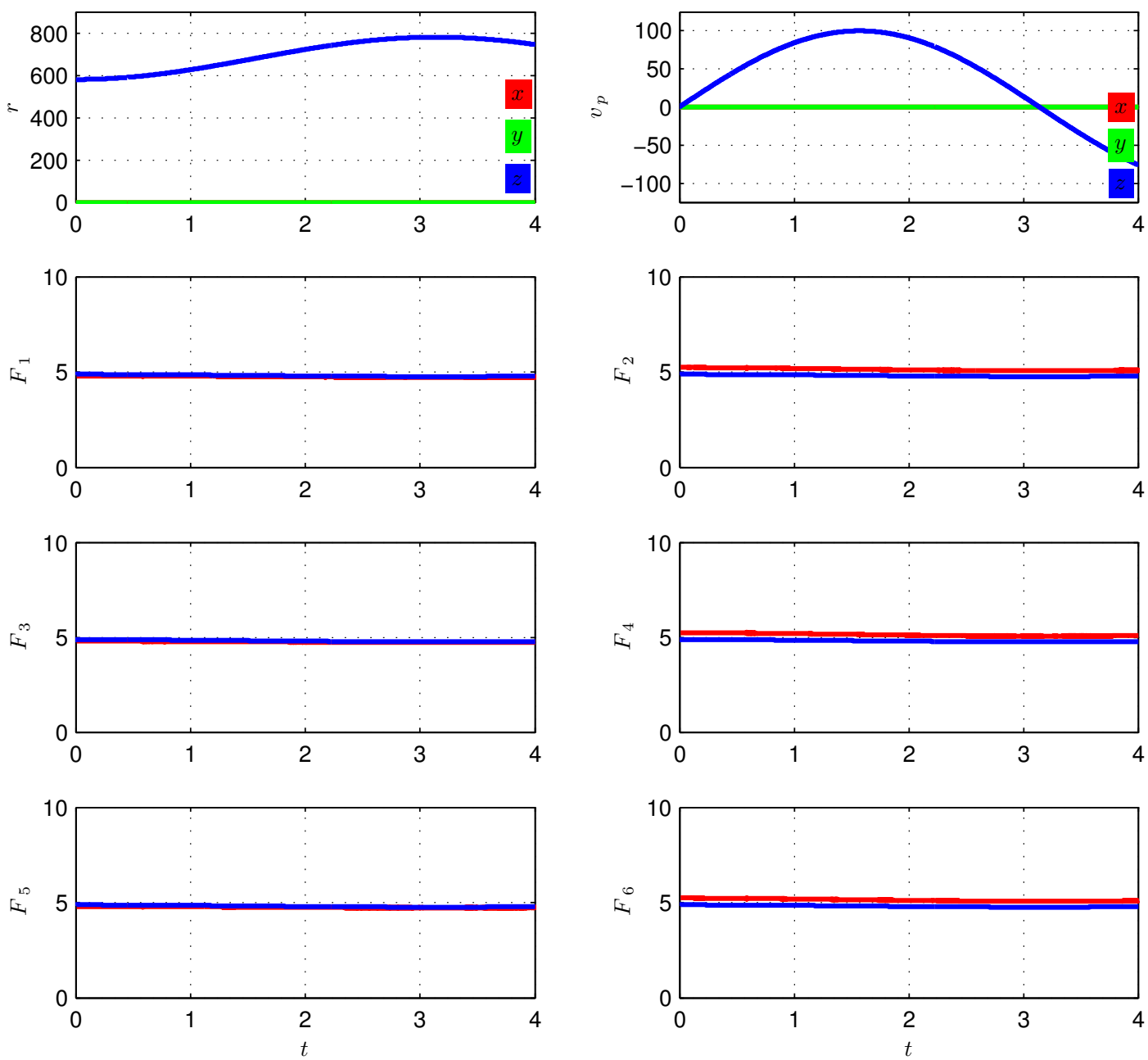

Figure E.3: Dynamic Model Validation, trajectory Tz. 

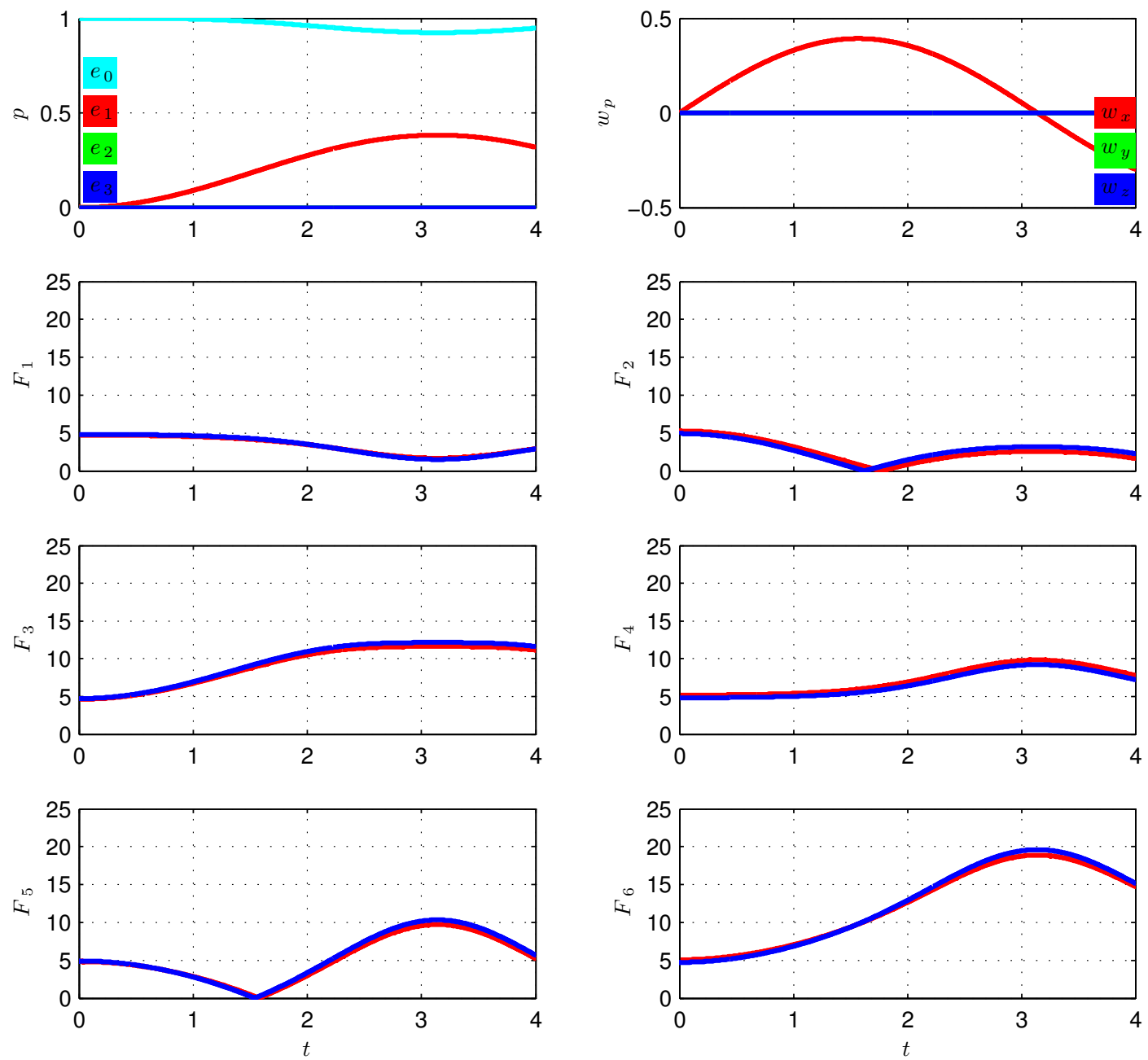

Figure E.4: Dynamic Model Validation, trajectory Rx. 

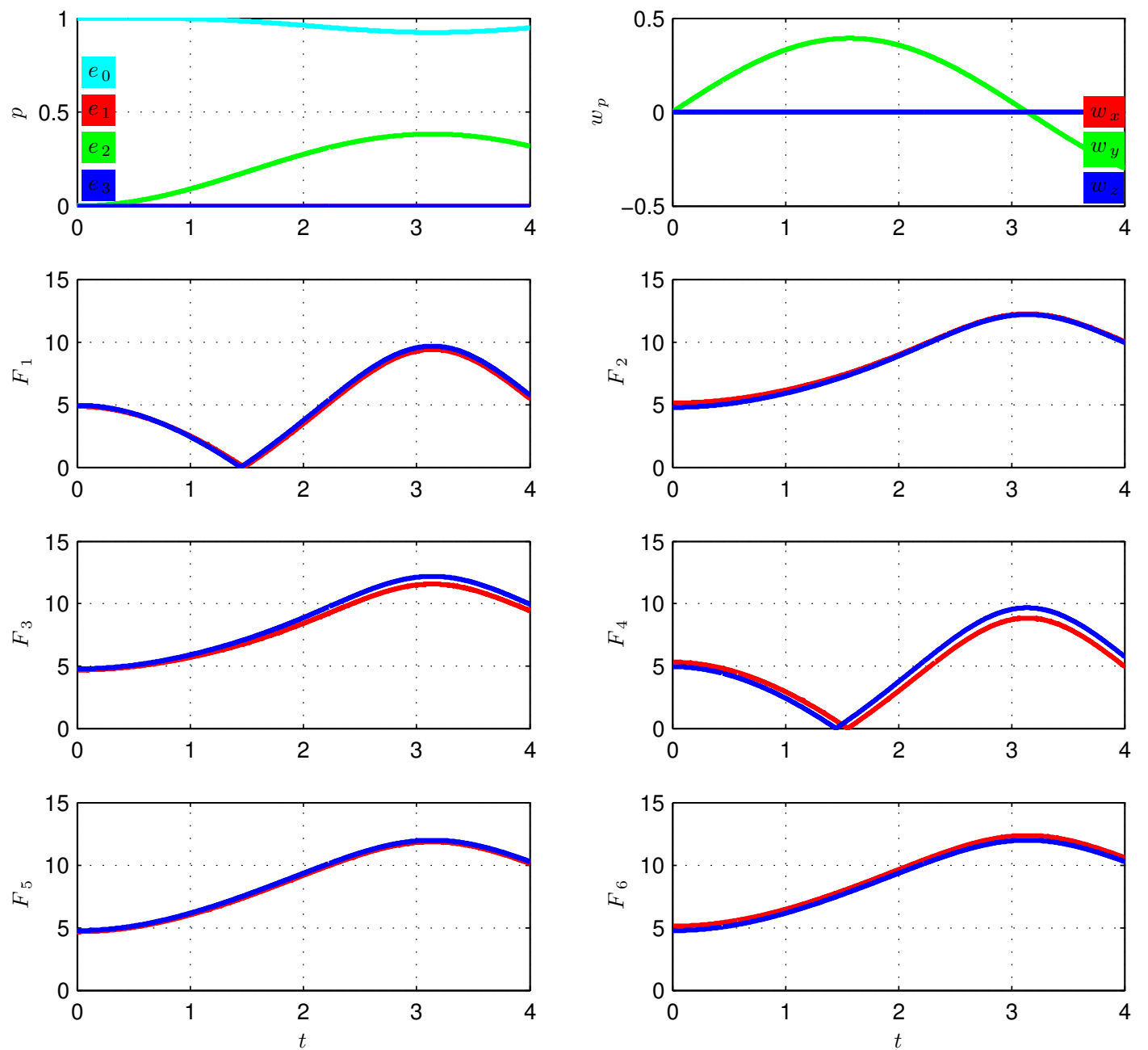

Figure E.5: Dynamic Model Validation, trajectory Ry. 

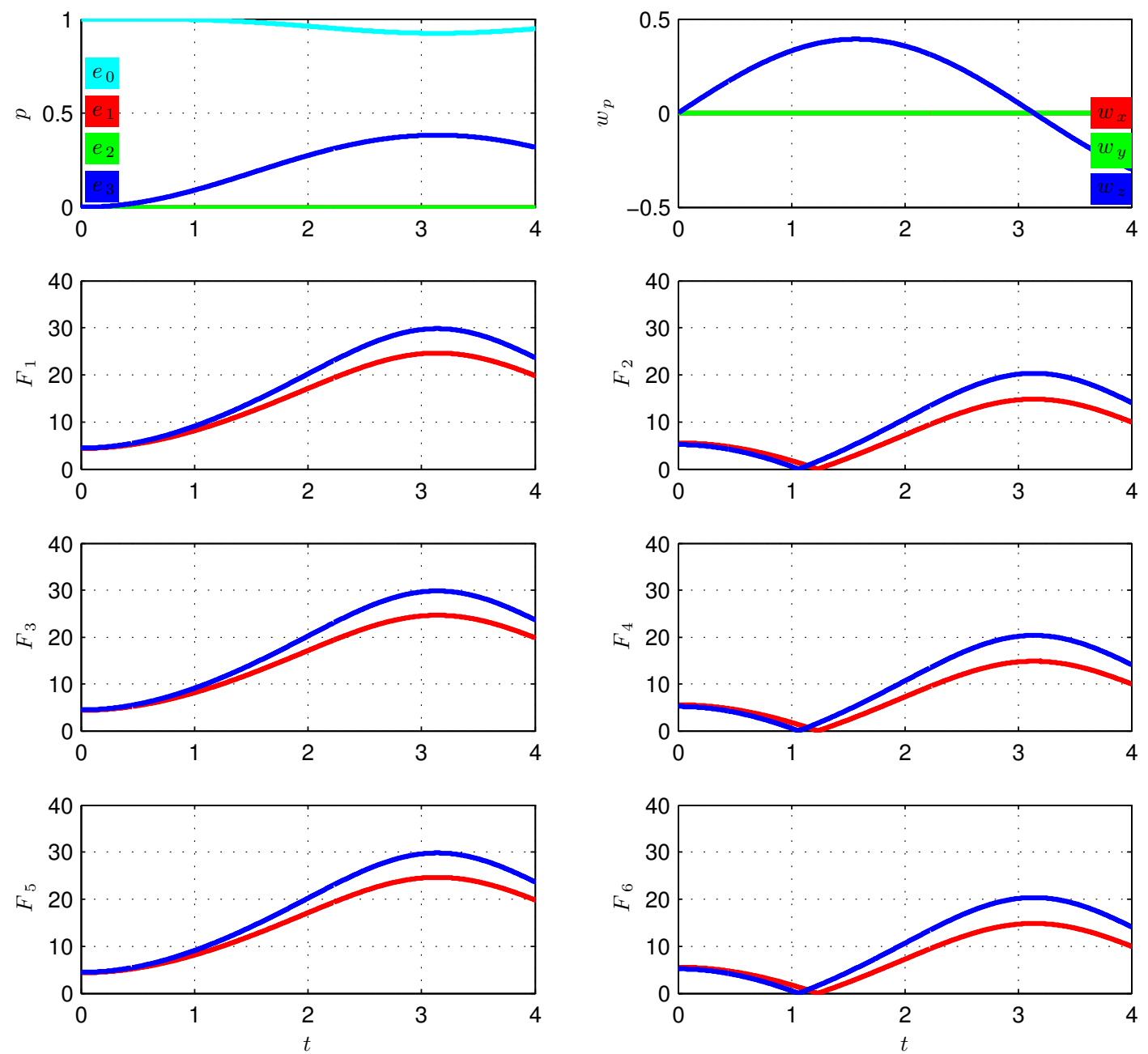

Figure E.6: Dynamic Model Validation, trajectory Rz. 
E. RESULTS FROM THE VALIDATION OF THE DYNAMIC MODEL OF THE PM 


\section{Bibliography}

[1] Alireza Akbarzadeh and Javad Enferadi. A Virtual Work Based Algorithm for Solving Direct Dynamics Problem of a 3-RRP Spherical Parallel Manipulator. Journal of Intelligent \& Robotic Systems, 63(1):25-49, November 2011. 149

[2] Farid Al-Bender and Jan Swevers. Characterization of friction force dynamics. IEEE Control Systems Magazine, 28(6):64-81, December 2008. 70

[3] Mazin I. Al-saedi, Huapeng Wu, and Heikki Handroos. Intelligent controller of a flexible hybrid robot machine for $\{$ ITER $\}$ assembly and maintenance. Fusion Engineering and Design, 89(7?8):1795 - 1803, 2014. Proceedings of the 11th International Symposium on Fusion Nuclear Technology-11 (ISFNT-11) Barcelona, Spain, 15-20 September, 2013. 160

[4] Stephan Algermissen and Michael Sinapius. Robust gain scheduling for smartstructures in parallel robots. In Daniel Schtz and FriedrichM. Wahl, editors, Robotic Systems for Handling and Assembly, volume 67 of Springer Tracts in Advanced Robotics, pages 159-174. Springer Berlin Heidelberg, 2011. 160

[5] Andrew Alleyne and J.K. Hedrick. Nonlinear adaptive control of active suspensions. IEEE Transactions on Control Systems Technology, 3(1):94-101, March 1995. 86

[6] Andrew Alleyne and Rui Liu. A simplified approach to force control for electrohydraulic systems. Control Engineering Practice, 8(12):1347-1356, December 2000. 86

[7] Andrew Alleyne and Heather Wright. On the limitations of force tracking control for hydraulic active suspensions. In Proceedings of the 1998 American Control Conference. ACC (IEEE Cat. No.98CH36207), volume 1, pages 43-47. American Autom. Control Council, 1998. 86 


\section{BIBLIOGRAPHY}

[8] Miguel Almonacid, Roque J. Saltaren, Rafael Aracil Santonja, and O. Reinoso. Motion planning of a climbing parallel robot. IEEE Transactions on Robotics and Automation, 19(3):485-489, 2003. 15, 121, 129

[9] Miguel Almonacid Kroeger. Modelling, Simulation and Control of a Parallel Climbing Robot. Ph.d, Universidad Miguel Hernández de Elche, Elche, Spain, 2002. 15

[10] Friedhelm Altpeter. FRICTION MODELING, IDENTIFICATION AND COMPENSATION. Phd, École Polytechnique Fédérale de Lausanne, 1999. 73

[11] Oscar Altuzarra, Asier Zubizarreta, Itziar Cabanes, and Charles Pinto. Dynamics of a four degrees-of-freedom parallel manipulator with parallelogram joints. Mechatronics, 19(8):1269-1279, December 2009. 148

[12] Rafael Aracil Santonja, Roque J. Saltaren, and O. Reinoso. A climbing parallel robot: a robot to climb along tubular and metallic structures. IEEE Robotics 8 Automation Magazine, 13(1):16-22, March 2006. 15, 121, 123

[13] T. Arai and K. Cleary. Design, analysis and construction of a prototype parallel link manipulator. In IEEE, editor, Proc. of IEEE Int. Conf. on intelligent robot and systems, volume 1, pages 205-212, 1990. 17

[14] Brian Armstrong and Qunyi Chen. The Z-properties chart. IEEE Control Systems Magazine, 28(5):79-89, October 2008. 70

[15] Brian Armstrong-Hélouvry, Pierre Dupont, and Carlos Canudas De Wit. A survey of models, analysis tools and compensation methods for the control of machines with friction. Automatica, 30(7):1083-1138, July 1994. 69

[16] Cesar Alvarez Arocha. Concepción y desarrollo de un vehículo submarino robótico de estructura paralela de geometría variable. Phd., Universidad Politécnica de Madrid, 2008. 15

[17] Johan Karl Astrom and Carlos Canudas-de Wit. Revisiting the LuGre friction model. IEEE Control Systems Magazine, 28(6):101-114, December 2008. 78, 80

[18] Karl J. Astrom and T. Hagglund. Pid controllers: theory, design and tuning. Instrument Society of America, 1995. 84

[19] J. Awrejcewicz and P. Olejnik. Analysis of Dynamic Systems With Various Friction Laws. Applied Mechanics Reviews, 58(6):389, 2005. 70 
[20] Robert S.. Ball. A Treatise on the Theory of Screws. Cambridge University Press, 1900. 19

[21] Ilka Banfield, Roque J. Saltaren, Lisandro J. Puglisi, and Rafael Aracil Santonja. Hybrid Position-Force Control of Climbing Parallel Robot Using Electrohydraulic Servo Actuators. In ASME 2011 International Design Engineering Technical Conferences $\&$ Computer and Information in Engineering Conference. IDETC/CIE 2011, Washington, DC, USA, 2011. 15, 87, 121, 122

[22] Ilka Banfield, Roque J. Saltaren, Lisandro J. Puglisi, Cecilia Garcia Cena, Ivan Pena Regueiro, Rafael Aracil, and Manuel N. Cardona. In CONCAPAN XXXI, IEEE, Salvador. 121, 124

[23] N. Barahanov and Romeo Ortega. Necessary and sufficient conditions for passivity of the LuGre friction model. IEEE Transactions on Automatic Control, 45(4):830-832, April 2000. 80

[24] P.R. Belanger. Estimation of angular velocity and acceleration from shaft encoder measurements. In Proceedings 1992 IEEE International Conference on Robotics and Automation, pages 585-592. IEEE Comput. Soc. Press, 1992. 109

[25] T X Bo. Sliding Mode Control of a Single Rigid Hydraulically Actuated Manipulator. International Journal of Mechanical \& Mechatronics Engineering, 11(5):1-9, 2011. 86

[26] J.E. Bobrow and K Lum. Adaptive, high bandwidth control of a hydraulic actuator. In Proceedings of 1995 American Control Conference - ACC'95, pages 71-75. American Autom Control Council, 1995. 85

[27] R F Boian, M Bouzit, G C Burdea, and J E Deutsch. Dual Stewart platform mobility simulator. Conference proceedings : ... Annual International Conference of the IEEE Engineering in Medicine and Biology Society. IEEE Engineering in Medicine and Biology Society. Conference, 7:4848-51, January 2004. 15

[28] Quentin Bombled and Olivier Verlinden. Dynamic simulation of six-legged robots with a focus on joint friction. Multibody System Dynamics, March 2012. 69

[29] A Bonchis, P I Corke, D C Rye, and Q P Ha. Variable structure methods in hydraulic servo systems control. Automatica, 37(2001):589-595, 2001. 85

[30] I. Bonev. The true origins of parallel robots, January 2003. 11 


\section{BIBLIOGRAPHY}

[31] I.A. Bonev, Damien Chablat, and P. Wenger. Working and assembly modes of the agile eye. Proceedings 2006 IEEE International Conference on Robotics and Automation, 2006. ICRA 2006., (May):2317-2322, 2006. 17

[32] I.A. Bonev and J. Ryu. A simple new closed-form solution of the direct kinematics using three linear extra sensors. In IEEE, editor, IEEE/ASME Int. Conf. on advanced intelligent mechanisms, volume 1, pages 526-530, 1999. 17

[33] Roger Boudreau, S. Darenfed, and C.M. Gosselin. On the computation of the direct kinematics of parallel manipulators using polynomial networks. IEEE Transactions on Systems, Man, and Cybernetics - Part A: Systems and Humans, 28(2):213-220, March 1998. 17

[34] Roger Boudreau and Noyan Turkkan. Solving the forward kinematics of parallel manipulators with a genetic algorithm. Journal of Robotic Systems, 13(2):111125, 1996. 17

[35] P J Costa Branco and J A Dente. On Using Fuzzy Logic to Integrate Learning Mechanisms in an Electro-Hydraulic System Part II : Actuator s Position Control. IEEE Transaction on Systems, Man, and Cybernetics - Part C: Applications and Reviews, 30(3):317-328, 2000. 38

[36] Alexandre Campos, Jacqueline Quintero, Roque J. Saltaren, Manuel Ferre, and Rafael Aracil Santonja. An Active helideck testbed for floating structures based on a Stewart-Gough platform. In 2008 IEEE/RSJ International Conference on Intelligent Robots and Systems, pages 3705-3710. IEEE, September 2008. 15

[37] C. Canudas de Wit, Henrik Olsson, K.J. Astrom, and P. Lischinsky. A new model for control of systems with friction. IEEE Transactions on Automatic Control, 40(3):419-425, March 1995. 72

[38] C. Canudas-de Wit, F.R. Rubio, and M.A. Corchero. D-OSKIL: A New Mechanism for Controlling Stick-Slip Oscillations in Oil Well Drillstrings. IEEE Transactions on Control Systems Technology, 16(6):1177-1191, November 2008. 69

[39] Rohitash Chandra and Luc Rolland. On solving the forward kinematics of 3RPR planar parallel manipulator using hybrid metaheuristics. Applied Mathematics and Computation, 217(22):8997-9008, July 2011. 17 
[40] Panagiotis Chatzakos and Evangelos Papadopoulos. ON MODEL-BASED CONTROL OF HYDRAULIC ACTUATORS. In 12th International Workshop on Robotics in Alpe-Adria-Danube Region, RADD'03, Cassino, 2003. 38

[41] Hong-Ming Chen, Jyh-Chyang Renn, and Juhng-Perng Su. Sliding mode control with varying boundary layers for an electro-hydraulic position servo system. The International Journal of Advanced Manufacturing Technology, 26(1-2):117-123, January 2005. 38, 85

[42] Jih-Hua Chin, Yen-His Sun, and Yuan-Ming Cheng. Force computation and continuous path tracking for hydraulic parallel manipulators. Control Engineering Practice, 16(6):697 - 709, 2008. Special Section on Large Scale Systems10th IFAC/IFORS/IMACS/IFIP Symposium on Large Scale Systems: Theory and Applications. 160

[43] Reymond Clavel. Conception d'un Robot Parallele Rapide a 4 Degres de Liberte. Phd, Ecole Polytechnique Federale de Lausanne, 1991. 12

[44] P.J. Costa Branco and J.A. Dente. On using fuzzy logic to integrate learning mechanisms in an electro-hydraulic system. II. Actuator's position control. IEEE Transactions on Systems, Man and Cybernetics, Part C (Applications and Reviews), (3):317-328, 2000. 86

[45] Bashkar Dasgupta and T S Mruthyunjaya. A Newton-Euler formulation for the inverse dynamics of the Stewart platform manipulator. Mechanism and Machine Theory, 33(8), 1998. 148

[46] Bhaskar Dasgupta and Prasun Choudhury. A general strategy based on the Newton - Euler approach for the dynamic formulation of parallel manipulators. Mechanism and Machine Theory, 34:94-114, 1999. 148

[47] K Dasgupta and H Murrenhoff. Modelling and dynamics of a servo-valve controlled hydraulic motor by bondgraph. Mechanism and Machine Theory, 46(7):1016-1035, July 2011. 38

[48] Ioannis Davliakos and Evangelos Papadopoulos. Model-based control of a 6dof electrohydraulic StewartGough platform. Mechanism and Machine Theory, 43(11):1385 - 1400, 2008. 160

[49] Ku Der-Ming. Direct displacement analysis of a stewart platform mechanism. Mechanism and Machine Theory, 34 (3):453-465, 1999. 130 


\section{BIBLIOGRAPHY}

[50] P Dietmaier. The stewart-gough platform of general geometry can have 40 real postures. In Advances in Robots Kinematics: Analysis and Control, pages 7-16. Kluwer Academic Publishers, 1998. 17

[51] Zhansheng Duan, Chongzhao Han, and H. Dang. An Adaptive Kalman Filter with Dynamic Rescaling of Process Noise. In Information Fusion, 2003. Proceedings of the Sixth International Conference of, number 3, pages 1310-1315, Cairns, Queensland, Australia, 2003. IEEE. 109

[52] G Dunlop. Position analysis of a 3-DOF parallel manipulator. Mechanism and Machine Theory, 32(8):903-920, November 1997. 17

[53] Roy Featherstone. Rigid Body Dynamics Algorithms. Springer US, Boston, MA, 2008. 148

[54] Leonid Freidovich, Anders Robertsson, Anton Shiriaev, and Rolf Johansson. LuGre-Model-Based Friction Compensation. IEEE Transactions on Control Systems Technology, 18(1):194-200, January 2010. 86

[55] J. Gallardo, J.M. Rico, A. Frisoli, D. Checcacci, and M. Bergamasco. Dynamics of parallel manipulators by means of screw theory. Mechanism and Machine Theory, 38(11):1113 - 1131, 2003. 149

[56] H. Garnier and Liuping Wang. Identification of Continuous-time Models form Sample Data. Advances in Industrial Control. Springer, 2008. 106

[57] G. Gogu. Structural Synthesis of Parallel Robots Part 2: Translational Topologies with Two and Three Degrees of Freedom. Solid Mechanics and Its Applications. Springer US, 2009. 9

[58] Oriol Gomis-Bellmunt, Flavio Campanile, Samuel Galceran-Arellano, Daniel Montesinos-Miracle, and Joan Rull-Duran. Hydraulic actuator modeling for optimization of mechatronic and adaptronic systems. Mechatronics, 18(10):634-640, December 2008. 38

[59] Dušan Gordić, Milun Babić, and Nebojša Jovičić. Modelling of spool position feedback servovalves. International Journal of Fluid Power, 5(1):37-50, 2004. 38

[60] C.M. Gosselin. Parallel computational algorithms for the kinematics and dynamics of planar and spatial parallel manipulators. Transaction of ASME, Journal of Dynamic Systems, Measurement and Control,, 118(1):22-28, 1996. 16, 148 
[61] Cheng Guan and Shuangxia Pan. Adaptive sliding mode control of electrohydraulic system with nonlinear unknown parameters. Control Engineering Practice, 16(11):1275-1284, November 2008. 86

[62] H B Guo and H R Li. Dynamic analysis and simulation of a six degree of freedom Stewart platform manipulator. Proceedings of the Institution of Mechanical Engineers, Part C: Journal of Mechanical Engineering Science, 220(1):61-72, January 2006. 149

[63] HongBo Guo, YongGuang Liu, GuiRong Liu, and HongRen Li. Cascade control of a hydraulically driven 6-DOF parallel robot manipulator based on a sliding mode. Control Engineering Practice, 16(9):1055 - 1068, 2008. 160

[64] Avraham Harnoy, Bernard Friedland, and Simon Cohn. Modeling and measuring friction effects. IEEE Control Systems Magazine, 28(6):82-91, December 2008. 73

[65] J.P. Hathout, J. Ahmed, and A. Kojic. Reduced order modeling and control of an electro-hydraulic valve system. In Proceedings of the 4 th IFAC Symposium on Advanced and Automatic Control 2004, volume 1, pages 185-187. Elsevier, 2004. 38

[66] Edward J. Haug. Computer Aided Kinematics and Dynamics of Mechanical Systems. Ally and Bacon, 1989. 135

[67] E.J. Haugh. Computer aided kinematics and dynamics of mechanical systems. Allyn and Bacon, 1989. 177

[68] B Heinrichs, N Sepehri, and A B Thornton-Trump. Position-Based Impedance Control of an Industrial Hydraulic Manipulator. IEEE Control Systems, (1):4652, 1997. 86

[69] Brian R Hopkins and Robert L. Williams II. Kinematics, design and control of the 6-PSU platform. Industrial Robot: An International Journal, 29(5):443-451, 2002. 130

[70] C.-L. Hwang. Sliding mode control using time-varying switching gain and boundary layer for electrohydraulic position and differential pressure control. IEE Proceedings - Control Theory and Applications, 143(4):325, 1996. 87 


\section{BIBLIOGRAPHY}

[71] P.K. Jamwal, S.Q. Xie, Y.H. Tsoi, and K.C. Aw. Forward kinematics modelling of a parallel ankle rehabilitation robot using modified fuzzy inference. Mechanism and Machine Theory, 45:1537-1554, 2010. 17

[72] Mohieddine Jelali and Andreas Kroll. Hydraulic Servo-systems. Modelling, Identification and Control Series: Advances in Industrial Control. Springer, 1st edtion edition, 2002. 27, 31, 33, 38, 40, 41, 50, 90

[73] Mohieddine Jelali and Helmut Schwarz. Nonlinear Identification of Hydraulic Servo-Drive Systems. IEEE Control Systems, (October), 1995. 54

[74] Ping Ji and Hongtao Wu. Kinematics analysis of an offset 3-UPU translational parallel robotic manipulator. Robotics and Autonomous Systems, 42(2):117-123, February 2003. 17

[75] Yao Jin and Fang Hai-rong. Forward displacement analysis of the decahedral variable geometry truss manipulator. Robotics and Autonomous Systems, 15(3):173178, August 1995. 17

[76] Claude Kaddissi, J.-P. Kenne, and M. Saad. Identification and Real-Time Control of an Electrohydraulic Servo System Based on Nonlinear Backstepping. IEEE/ASME Transactions on Mechatronics, 12(1):12-22, February 2007. 87

[77] M. Karpenko and N. Sepehri. Fault-tolerant control of a servohydraulic positioning system with crossport leakage. IEEE Transactions on Control Systems Technology, 13(1):155-161, January 2005. 38

[78] Wisama Khalil and Ouarda Ibrahim. General Solution for the Dynamic Modeling of Parallel Robots. Journal of Intelligent and Robotic Systems, 49(1):19-37, March 2007. 148

[79] Karim Khayati, Pascal Bigras, and Louis-A. Dessaint. LuGre model-based friction compensation and positioning control for a pneumatic actuator using multi-objective output-feedback control via LMI optimization. Mechatronics, 19(4):535-547, June 2009. 86

[80] H Kim, Y Cho, and K Lee. Robust nonlinear task space control for 6 DOF parallel manipulator. Automatica, 41(9):1591-1600, September 2005. 160

[81] Xianwen Kong and Clement M. Gosselin. Type Synthesis of Parallel Mechanisms, volume 33. Springer, 2007. 10, 19 
[82] Laurent Laval, N.K. M'Sirdi, and J.-C. Cadiou. H/sub /-force control of a hydraulic servo-actuator with environmental uncertainties. In Proceedings of IEEE International Conference on Robotics and Automation, volume 2, pages 15661571. IEEE, 1996. 86

[83] K.-M. Lee and D.K. Shah. Dynamic analysis of a three-degrees-of-freedom in-parallel actuated manipulator. IEEE Journal on Robotics and Automation, 4(3):361-367, June 1988. 148

[84] Se-Han Lee, Jae-Bok Song, Woo-Chun Choi, and Daehie Hong. Position control of a Stewart platform using inverse dynamics control with approximate dynamics. Mechatronics, 13(6):605 - 619, 2003. 160

[85] Geqiang Li, Hanjie Wang, Xiaodong Huang, and Hongyan Yang. Research on the Control Scheme of Direct Drive Electro-hydraulic Position Servo System. pages 3221-3224, 2011. 26

[86] Lei Li, Qidan Zhu, and Liyan Xu. Solution for forward kinematics of 6-dof parallel robot based on particle swarm optimization. In Proceedings of the 2007 IEEE International Conference on Mechatronics and Automation, 2007 ( 2968-2973). 17

[87] Hsien-hsin Liao, Matthew J Roelle, Jyh-shin Chen, Sungbae Park, and J Christian Gerdes. Implementation and Analysis of a Repetitive Controller for an Electro-Hydraulic Engine Valve System. IEEE Transactions on Control Systems Technology, 19(5):1102-1113, September 2011. 54

[88] T. G. Ling, M. F. Rahmat, a. R. Husain, and R. Ghazali. System identification of electro-hydraulic actuator servo system. 2011 4th International Conference on Mechatronics (ICOM), (May):1-7, May 2011. 54

[89] P. Lischinsky, C. Canudas-de Wit, and G. Morel. Friction compensation for an industrial hydraulic robot. IEEE Control Systems Magazine, 19(1):25-32, 1999. 73,105

[90] G.P. Liu and S. Daley. Optimal-tuning PID controller design in the frequency domain with application to a rotary hydraulic system. Control Engineering Practice, 7(7):821-830, July 1999. 54 


\section{BIBLIOGRAPHY}

[91] Min-jie Liu, Cong-xin Li, and Chong-ni Li. Dynamics analysis of the GoughStewart platform manipulator. IEEE Transactions on Robotics and Automation, 16(1):94-98, 2000. 84, 148

[92] Y. Liu and H. Handroos. Technical note Sliding mode control for a class of hydraulic position servo. Mechatronics, 9(1):111-123, February 1999. 85

[93] Lennart Ljung. System Identification Toolbox User's Guide. The Math Works Inc., 2002. 57

[94] O. Ma and Jorge Angeles. Optimum architecture design of platform manipulators. IEEE, 1991. 21

[95] Mehdi Tale Masouleh, Clement M. Gosselin, Manfred Husty, and Dominic R. Walter. Forward kinematic problem of 5-RPUR parallel mechanisms (3T2R) with identical limb structures. Mechanism and Machine Theory, 46(7):945-959, July 2011. 17

[96] J. P. Merlet. Solving the Forward Kinematics of a Gough-Type Parallel Manipulator with Interval Analysis. The International Journal of Robotics Research, 23(3):221-235, March 2004. 17

[97] Jean Pierre Merlet. Direct kinematics of planar parallel manipulators. In Proceedings of IEEE International Conference on Robotics and Automation, volume 4, pages 3744-3749. IEEE, 1996. 17

[98] Jean Pierre Merlet. Parallel Robots, volume 128 of Solid Mechanics and Its Applications. Springer-Verlag, Berlin/Heidelberg, 2nd edition, 2006. 16, 148

[99] Jean Pierre Merlet and D Daney. Legs Interference Checking of Parallel Robots over a Given Workspace or Trajectory. In Robotics and Automation, number May, pages 757-762, Orlando, Florida, 2006. 131

[100] J.P. Merlet. Parallel Robots. 2nd.ed., volume 1. Springer, 2006. 17, 129

[101] H.E. Merrit. Hydraulic Control Systems. John Wiley \& Sons, 1967. 27, 38

[102] Amit Mohanty and Bin Yao. Indirect Adaptive Robust Control of Hydraulic Manipulators With Accurate Parameter Estimates. IEEE Transactions on Control Systems Technology, 19(3):567-575, May 2011. 85 
[103] Amit Mohanty and Bin Yao. Integrated Direct/Indirect Adaptive Robust Control of Hydraulic Manipulators With Valve Deadband. IEEE/ASME Transactions on Mechatronics, 16(4):707-715, August 2011. 85

[104] Industrial Control Division MOOG. Electrohydraulic valves... a technical look. Technical report. 38

[105] Hector A Moreno, Lisandro J. Puglisi, Roque J. Saltaren, and Isela Carrera. Kinematic analysis of an Underwater Parallel Robot. In OCEANS 2011 IEEE Spain, pages 1-6. IEEE, June 2011. 15

[106] Héctor a. Moreno, Roque Saltaren, Isela Carrera, Lisandro Puglisi, and Rafael Aracil. İndices de Desempeño de Robots Manipuladores: una revisión del Estado del Arte. Revista Iberoamericana de Automática e Informática Industrial RIAI, 9(2):111-122, April 2012. 21

[107] Samir Nabulsi, J.F. Sarria, Hector Montes, and M.A. Armada. High-Resolution Indirect FeetGround Interaction Measurement for Hydraulic-Legged Robots. IEEE Transactions on Instrumentation and Measurement, 58(10):3396-3404, October 2009. 33

[108] P. Nanua, K.J. Waldron, and V. Murthy. Direct kinematic solution of a Stewart platform. IEEE Transactions on Robotics and Automation, 6(4):438-444, 1990. 17

[109] Jose Sabater Navarro, Nicolas Garcia, Carlos Perez, Eduardo Fernandez, Roque J. Saltaren, and Miguel Almonacid. Kinematics of a robotic 3UPS1S spherical wrist designed for laparoscopic applications. The international journal of medical robotics + computer assisted surgery : MRCAS, 6(3):291-300, September 2010. 16

[110] N. Niksefat and Nariman Sepehri. Designing robust force control of hydraulic actuators despite system and environmental uncertainties. IEEE Control Systems Magazine, 21(2):66-77, April 2001. 87

[111] Ka-tjun Oen and L. Wang. Extreme Reaches and Maximal Reachable Workspace for Rotary Tools Mounted on a Stewart Platform Manipulator. JOURNALCHINESE INSTITUTE OF ENGINEERS, 29(6):967, 2006. 131

[112] H. Olsson and K.J. Astrom. Friction generated limit cycles. IEEE Transactions on Control Systems Technology, 9(4):629-636, July 2001. 69 


\section{BIBLIOGRAPHY}

[113] Henrik Olsson. Control Systems with friction. PhD thesis, Lund Institute of Technology, June 1996. 71, 72, 77

[114] Henrik Olsson, Johan Karl Astrom, Carlos Canudas-de Wit, M. Gafvert, and P. Lischinsky. Friction Models and Friction Compensantion.pdf. European Journal of Control, 4(3):176-195, 1998. 70

[115] A. Omran, M. Bayoumi, A. Kassem, and G. El-Bayoumi. Optimal Forward Kinematics Modeling of Stewart Manipulator Using Genetic Algorithms. Jordan Journal of Mechanical and Industrial Engineering, 3(4):280-293, 2009. 17

[116] W.S. Owen and E.A. Croft. The reduction of stick-slip friction in hydraulic actuators. IEEE/ASME Transactions on Mechatronics, 8(3):362-371, September 2003. 70

[117] A. Padthe, B. Drincic, D. Rizos, S. Fassois, and D. Bernstein. Duhem modeling of friction-induced hysteresis. IEEE Control Systems Magazine, 28(5):90-107, October 2008. 69

[118] Pratik J. Parikh and Sarah S. Lam. Solving the forward kinematics problem in parallel manipulators using an iterative artificial neural network strategy. The International Journal of Advanced Manufacturing Technology, 40(5-6):595-606, January 2008. 17

[119] G. R. Pennock and D. J. Kassner. The workspace of a general geometry planar three-degree-of-freedom platform-type manipulator. Journal of Mechanical Design, 115(2):269-276, 1993. 17

[120] Lisandro J. Puglisi, Roque J. Saltaren, and C. Garcia. On the Velocity and Accelertation Estimation from Discrete Time-Position Sensors. Control Engineering and Applied Informatics, pages 30-40, 2015. 108

[121] Lisandro J. Puglisi, Roque J. Saltaren, Hector a. Moreno, Pedro F. Cárdenas, Cecilia E. Garcia Cena, and Rafael Aracil. Dimensional synthesis of a spherical parallel manipulator based on the evaluation of global performance indexes. Robotics and Autonomous Systems, 60(8):1037-1045, August 2012. 21

[122] Lisandro J. Puglisi, Roque J. Saltaren, Germán Rey Portolés, Hector Moreno, Pedro F. Cárdenas, and Cecilia Garcia. Design and kinematic analysis of 3PSS-1S wrist for needle insertion guidance. Robotics and Autonomous Systems, 61(5):417427, May 2013. 16, 123, 144 
[123] M. Galal Rabie. Fluid Power Engineering. McGraw-Hill, 2009. 28

[124] M. Raghavan. The stewart platform of general geometry has 40 configurations. Jounal of Mechanical Design, 115(2):277-282, 1993. 17

[125] M.F. Rahmat, A R Husain, K Ishaque, Y M Sam, Rozaimi Ghazali, and S Rozali. Modeling and controller design of an industrial hydraulic actuator system in the presence of friction and internal leakage. International Journal of the Physucal Sciences, 6(14):3502-3517, 2011. 85

[126] M.F. Rahmat, S. Rozali, N.A. Wahab, Zulfatman, and J. Kamaruzaman. Modeling and Controller Design of an Electro-Hydraulic Actuator System. American Journal of Applied Sciences, 7(8):1100-1108, August 2010. 49, 54, 102

[127] J.M. Sabater, R.J. Saltarén, and R. Aracil. Design, modelling and implementation of a 6 URS parallel haptic device. Robotics and Autonomous Systems, 47(1):1-10, May 2004. 15

[128] A. M. Sabatini. Real-time kalman filter applied to biomechanical data for state estimation and numerical differentiation. Medical \& Biological Engineering \& Computing, 41(1):2-10, January 2003. 109

[129] H. Sadjadian and H.D. Taghirad. Comparison of different methods for computing the forward kinematics of a redundant parallel manipulator. Journal of Intelligent and Robotic Systems, pages 10.1007/s10846-005-9006-4, 2006. 17

[130] R.J. Saltaren, R. Aracil, L.J. Puglisi, G. Poletti, G. Rey, and W. Cabrera. Robot de estructura cinemtica hbrida para el guiado de la insercin de agujas, catteres y elementos quirrgicos para procedimientos de ciruga mnimamente invasiva, 04 2013. 16

[131] Roque J. Saltaren, Rafael Aracil Santonja, C. Alvarez, Eugenio Yime, and J.M. Sabater-Navarro. Field and service applications - Exploring deep sea by teleoperated robot - An Underwater Parallel Robot with High Navigation Capabilities. IEEE Robotics \& Automation Magazine, 14(3):65-75, September 2007. 15

[132] Roque J. Saltaren, Rafael Aracil Santonja, O. Reinoso, and M.a. Scarano. Climbing parallel robot: a computational and experimental study of its performance around structural nodes. IEEE Transactions on Robotics, 21(6):1056-1066, December 2005. 15 


\section{BIBLIOGRAPHY}

[133] Maria Antonietta Scarano Roo. Espacio de Trabajo y Singularidades en Robots Paralelos: Desarrollo, Análisis y Aplicación al Robot Trepa. Ph.d, Universidad Politécnica de Madrid, Madrid, Spain, 2008. 15

[134] N Sepehri, T Corbet, and P D Lawrence. FUZZY POSITION CONTROL OF HYDRAULIC ROBOTS WITH VALVE DEADBANDS. 5(6):623-643, 1995. 84

[135] J.R. Serracín, L.J. Puglisi, R. Saltaren, G. Ejarque, J.M. Sabater-Navarro, and R. Aracil. Kinematic analysis of a novel 2-d.o.f. orientation device. Robotics and Autonomous Systems, 60(6):852-861, June 2012. 16, 123, 131

[136] Wang Shaowei and Wan Shanming. Velocity and acceleration computations by single-dimensional Kalman filter with adaptive noise variance. Przegld Elektrotechniczny, (2):283-287, 2012. 109

[137] Moshe Shoham, M. Burman, E. Zehavi, L. Joskowicz, E. Batkilin, and Y. Kunicher. Bone-mounted miniature robot for surgical procedures: concept and clinical applications. IEEE Transactions on Robotics and Automation, 19(5):893-901, October 2003. 15

[138] Klaus Six, T.A. Lasky, and Bahram Ravani. A time-delayed dynamic inversion scheme for mechatronic control of hydraulic systems. In 2001 IEEE/ASME International Conference on Advanced Intelligent Mechatronics. Proceedings (Cat. No.01TH8556), volume 2, pages 1232-1238. IEEE, 2001. 87

[139] Michael G. Skarpetis, F. N. Koumboulis, and M. P. Tzamtzi. Robust control techniques for hydraulic actuators. In 2007 Mediterranean Conference on Control E Automation, number 1, pages 1-6. IEEE, June 2007. 84

[140] G.A. Sohl and J.E. Bobrow. Experiments and simulations on the nonlinear control of a hydraulic servosystem. IEEE Transactions on Control Systems Technology, 7(2):238-247, March 1999. 87

[141] Se-Kyong Song and Dong-Soo Kwon. New Direct Kinematic Formulation of 6DOF Sewart-Gough Platform Using the Thetrahedron Approach. Transaction of Control, Automation, and Systems Engineering, 4(3):217-223, 2002. 17

[142] H. Sun and G.T.-C. Chiu. Nonlinear observer based force control of electrohydraulic actuators. In Proceedings of the 1999 American Control Conference (Cat. No. 99CH36251), volume 2, pages 764-768. IEEE, 1999. 86 
[143] S. Tafazoli, C.W. de Silva, and P.D. Lawrence. Tracking control of an electrohydraulic manipulator in the presence of friction. IEEE Transactions on Control Systems Technology, 6(3):401-411, May 1998. 86

[144] L. Tsai. Robot Analysis, The Mechanics of Serial and Parallel Manipulators. 1999. 17

[145] Lung Wen Tsai. Robot Analysis: the Mechanics of Serial and Parallel Manipulators. John Wiley \& Sons, 1999. 18, 23, 144, 145, 148, 149

[146] Lung-Wen Tsai. Solving the Inverse Dynamics of a Stewart-Gough Manipulator by the Principle of Virtual Work. Journal of Mechanical Design, 122(1):3-9, 2000. 149

[147] Jiegao Wang and Clément M Gosselin. A New Approach for the Dynamic Analysis of Parallel Manipulators. Multibody System Dynamics, 2:317-334, 1998. 149

[148] Xue-Song Wang, Ming-Lin Hao, and Yu-Hu Cheng. On the use of differential evolution for forward kinematics of parallel manipulators. Applied Mathematics and Computation, 205(2):760-769, November 2008. 17

[149] John Watton. Fluid Power Systems. Modeling, simulation, analog and microcomputer control. Prentice Hall International (UK) Ltd, 1989. 27, 38, 67

[150] Greg Welch and Gary Bishop. An Introduction to the Kalman Filter. In Lou Harrison, editor, 28th International Conference on Computer Graphics and Interactive Techniques -SIGGRAPH2001, pages 1-81, North Carolina, 2001. ACM, Inc. 109

[151] G. Wu, N. Sepehri, and K. Ziaei. Design of a hydraulic force control system using a generalised predictive control algorithm. IEE Proceedings - Control Theory and Applications, 145(5):428, 1998. 86

[152] Bin Yao, Fanping Bu, J. Reedy, and G.T.-C. Chiu. Adaptive robust motion control of single-rod hydraulic actuators: theory and experiments. IEEE/ASME Transactions on Mechatronics, 5(1):79-91, March 2000. 85

[153] E. Yime, Roque J. Saltaren, Cecilia E. Garcia Cena, and Jose Maria Sabater. Robot based on task-space dynamical model. IET Control Theory $\&$ Applications, 5(18):2111, 2011. 149 


\section{BIBLIOGRAPHY}

[154] Eugenio Yime Rodríguez. Modelado Matemático y Control Vectorial de Robots Submarinos de Goemtría Variable. Ph.d, Universidad Politécnica de Madrid, Madrid, Spain, 2008. 15

[155] Tienan Zhao and Tapio Virvalo. Development of fuzzy state controller and its application to a hydraulic position servo. Fuzzy Sets and Systems, 70(2-3):213221, March 1995. 84

[156] Jilin Zhou, Xiaojun Shen, Emil M. Petriu, and Nicolas D. Georganas. Linear velocity and acceleration estimation of 3 DOF haptic interfaces. In 2008 IEEE International Workshop on Haptic Audio visual Environments and Games, pages 137-142. IEEE, October 2008. 109

[157] Kemin Zhou and John C. Doyle. Essentials of Robust Control. Prentice Hall, 1999. $90,96,98$

[158] Dimiter Zlatanov, R.G. Fenton, and B. Benhabib. Singularity analysis of mechanisms and robots via a velocity-equation model of the instantaneous kinematics. IEEE Comput. Soc. Press. 144

[159] Asier Zubizarreta, Marga Marcos, Itziar Cabanes, Charles Pinto, and Eva Portillo. Redundant sensor based control of the 3RRR parallel robot. Mechanism and Machine Theory, 54:1-17, August 2012. 130

[160] Zulfatman and M.F. Rahmat. Application of Self Tuning Fuzzy PID Controller on Industrial Hydraulic Actuator. International Journal on Smart Sensing and Intelligent Systems, 2(2):246-261, 2009. 84 


\section{Declaration}

I herewith declare that I have produced this paper without the prohibited assistance of third parties and without making use of aids other than those specified; notions taken over directly or indirectly from other sources have been identified as such. This paper has not previously been presented in identical or similar form to any other German or foreign examination board. The thesis work was conducted from XXX to YYY under the supervision of PI at ZZZ.

CITY, 